\title{
Über solche algebraische Curven, welche einen Mit- telpunct haben, und über darauf bezügliche Eigen- schaften allgemeiner Curven, so wie über geradlinige Transversalen der letztern.
}

(Theils Auszug, theils Erweiterung eines am 26. Mai 1851 in der Akademie der Wissenschaften gehaltenen Vortrags.)

(Vom Herrn Professor J. Steiner, zu Berlin.)

\$. 1.
Die Curven zweiten Grads haben schon an sich Mittelpuncte, es ist eine ihnen inwohnende Eigenschaft. Anders verhält es sich mit den Curven höherer Ordnung. Wohl besitzen noch die Curven dritten Grads die Eigenschaft, dafs sie sich durch Projection in solche umwandeln lassen, welche Mittelpuncte haben; wogegen alle hŏheren Curven gewisse Beschränkungen zu erleiden haben, wenn ihnen die Eigenschaft eines Mittelpuncts zukommen soll.

Unter ,Mittelpunct" einer Curve $m^{\text {ten }}$ Grads, $C^{m}$, wird ein solcher in ihrer Ebene liegender Punct $M$ verstanden, welcher die Eigenschaft hat, dafs jede durch ihn gezogene unbegrenzte Gerade $\boldsymbol{S}$, die Curve in solchen $m$ Puncten schneidet, welche paarweise gleichweit von ihm abstehen, so dafs also die Schnittpuncte auf beiden Seiten von jenem Puncte $\mathfrak{B}$ gleich vertheilt sind, und jedem Punct $p$ auf der einen Seite ein anderer $p_{1}$ auf der entgegengesetzten Seite, in gleichem Abstande von $\mathfrak{M}$, entsprechen mufs und sein „Gegenpuncl" genannt wird. Hiernach möchle es scheinen, als könne eine Curve $C^{m}$ nur dann einen Mittelpunct $\mathfrak{M}$ haben, wenn ihr Gradexponent $m$ eine gerade Zahl ist, etwa $m=2 \mu$, weil nur dann in jeder Transversalen $\mathbb{S}$ zu beiden Seiten von $\mathfrak{M}$ gleichviel Schnitte liegen können, was dagegen, wenn $m$ ungerade, $m=2 \nu-1$, nicht mōglich ist. Indessen wird dieser scheinbare Einwand dadurch beseitigt, dafs im letztern Falle ein einzelner Schinittpunct im Mittelpuncte $\mathfrak{M}$ selbst liegt, somit ein Zweig der Curve $\boldsymbol{C}^{\mathbf{Q}_{\nu-1}}$ durch ihren Mittelpunct selbst geht, wobei alsdann auf jeder Seite von diesem noch $\nu-1$ Schnitte liegen, die sich paarweise als Gegenpuncte entsprechen; jener besondere Punct aber mufs nothwendig ein Wendepunct der Curve $C^{2 v-1}$ sein. 
In besondern Fällen kann die Curve $C^{m}$ auch öfter durch ihren eigenen Mittelpunct $\mathfrak{M}$ gehen, und zwar verhält es sich damit, wie folgt. Ist $m=2 \mu$, so können insbesondere gleichzeitig 2 , oder 4 , oder 6 , etc. Zweige der Curve $C^{2 \mu}$ durch $\mathfrak{M}$ gehen, d. h. sie kann ihren Mittelpunct $\mathfrak{M}$ zugleich zum vielfachen Puncte haben, jedoch nur zum $2,4,6, \ldots, 2(\mu-1)$ fachen. Und ist $m=2 v-1$, so mufs nothwendig ein $\mathrm{Zweig}$ der Curve $C^{2 v-1}$ durch ihren Mittelpunct $\mathfrak{M}$ gehen, aber es können insbesondere auch $3,5,7, \ldots$ Zweige durch denselben gehen, wo er dann ein ebenso vielfacher Punct von ihr ist. In beidén Fällen sind die Tangenten im Mittelpuncte $\mathfrak{M}$ höherer Art, nämlich sie sind zugleich Wendetangenten der respectiven Zweige, und haben somit, wenn $x$ Zweige durch $\mathfrak{M}$ gehen, daselbst $x+2$ Puncte mit der Curve gemein, was als eine $(x+2)$ punctige Berührung anzusehen ist.

\section{S. 2 .}

Zur Bestimmung solcher Curven $\boldsymbol{C}^{\boldsymbol{m}}$, welche Mittelpuncte haben, durch gegebene Puncte, kann entweder 1) der Mittelpunct $\mathfrak{M}$ selbst gegeben werden und nebstdem noch eine genügende Anzahl andere Puncte $p$, durch welche die Curve gehen soll; oder es können 2) blos solche beliebige Puncte $p$, durch welche die Curve gehen soll, gegeben und dazu verlangt werden, dafs dieselbe einen Mittelpunct $\mathfrak{M}$ haben müsse, dessen Lage dann durch jene Puncte erst bedingt wird. Bei dieser Bestimmung, so wie schon vorhin (\$.1.) und auch in der Folge, macht sich der Umstand geltend, ob der Gradexponent in eine gerade oder eine ungerade Zahl, also ob $\alpha$ ) $m=2 \mu$, oder B) $m=2 v-1$ ist; denn danach scheiden sich die Sätze folgendermafsen:

„Ist der Mittelpunct $\mathfrak{M}$ gegeben, so ist

a) Die Curve $C^{2 \mu}$ bestimmt durch $(\mu+1)^{2}-1=\frac{1}{4} m(m+4)$,

B) Die Curve $C^{2 v-1}$ bestimmt durch $\nu(\nu+1)=\frac{1}{4}[m(m+4)-1]$ beliebige andere gegebene Puncte p, durch welche sie gehen soll." *)

*) Zur Bestimmung der Curven, welche Mittelpuncte haben, bietet die Gleichung dersejben ein anschauliches und bequemes Millel dar. Wenn nămlich die in beliebig schiefwinkligen Coordinaten, nach den Dimensionen der Veränderlichen $(x$ und $y$ ) geordnete Gleichung einer Curve $C^{m}$, von der höchsten Dimension abwärts nur die abwechselnden Dimensinnen enthält, alle übrigen gleich Null sind, wenn somit die Gleichung von der Form

$$
\boldsymbol{D}^{m}+\boldsymbol{D}^{m-2}+\boldsymbol{D}^{m-4}+\cdots \cdot \boldsymbol{D}^{m-2 \alpha+2}+\cdots=0
$$

ist, so hat die Curve allemal den Anfangspunct zugleich zu ihrem Mittelpuncte $\mathfrak{M}$. Werden 
D.a im Allgemeinen eine Curve $m^{\text {ten }}$ Grads durch

$$
\text { r) } \frac{1}{2} m(m+3)
$$

Puncle $p$ bestimmt wird, so sieht man, wieviele bestimmende Puncte $p$ durch den gegebénen Mittelpunct vertreten werden, nämlich

$$
\begin{aligned}
& \text { „Der Mittelpunct } \mathfrak{R} \text { vertritt } \\
& \alpha^{(1)} \text { bei } C^{2 \mu}: \quad \mu(\nu+1)=\frac{1}{4} m(m+2), \\
& \beta^{(j)} \text { bei } C^{2 v-1}: \quad \nu^{2}=\frac{1}{4}[m(m+2)+1]
\end{aligned}
$$

bestimmende Puncte p."

Aber der gegebene Mittelpunct bedingt noch mehr; denn mit ihm sind auch zugleich alle Gegenpuncte $p_{1}$ zu den gegebenen Puncten $p$ bestimmt oder als gegeben anzusehen, durch welche die Curve nothwendig ebenfalls geht, so dafs also zusammen bezieblich ( $\alpha$ und $\beta$ )

$$
\frac{1}{2} m(m+2) \text { und } \frac{1}{2}[m(m+2)+1]
$$

Puncte $p$ und $p_{1}$ gegeben sind, somit mehr, als die Beslimmung der Curve im Allgemeinen erheischt oder zuläfst $(\gamma)$, und zwar sind

$$
\begin{array}{ll}
\text { für } C^{2 \mu}: & \mu=\frac{1}{2} m, \\
\text { für } C^{2 \nu-1}: & \nu-1=\frac{1}{2}(m-1)
\end{array}
$$

Puncte mehr gegeben, ohne dafs dadurch die Curve überbestimmt wird. Den obigen Satz kann man danach auch so aussprechen:

,Sind $\mu(\mu+2)\left(=\frac{1}{4} m(m+4)\right)$, oder $\nu(\nu+1)-1\left(=\frac{1}{4}[m(m+4)-1]\right)$ beliebige begrenzte Gerade oder Sehnen pp gegeben, die alle durch den-

die zwei Zahlformen von $m$ unterschieden, so hat man folgende zwei Gleichungen:

$$
\begin{array}{rrrr}
\text { I. } & D^{2 \mu}+D^{2 \mu-2}+D^{2 \mu-4}+\cdots+D^{2}+D^{0}=0 \text {; für } \boldsymbol{C}^{2 \mu}, \\
\text { II. } & D^{2 \nu-1}+D^{2 \nu-3}+D^{2 \mu-5}+\cdots+D^{3}+D^{1}=0 \text {; für } C^{2 \nu-1} \text {. }
\end{array}
$$

Je nachdem also der Gradexponent gerad oder ungerad ist, enthält die Mittelpuncts-Gleichung der Curve $\left(^{m}\right.$ auch nur die Glieder von gerader oder ungerader Dimension, indem alle übrigen $=0$ sein müssen. In (I.) bezeichnet $\boldsymbol{D}^{0}$ das constante Glied. Dafs die Curve $C^{2 v-1}$ nothwendig durch ihren eigenen Mittelpunct geht, ist aus (II.) ersichtlich.

Da jede Dimension ein Glied mehr umfafst, als ihr Exponent anzeigt, z. B. da $D^{\alpha}$ die $\alpha+1$ Glieder

$$
y^{\alpha}, y^{\alpha-1} x, y^{\alpha-2} x^{2}, \ldots y^{\alpha-1} x^{\alpha},
$$

abgesehen von den Coëfficienten, umfafst, so ist die Zahl aller Glieder in den beiden Gleichungen

$$
\begin{aligned}
& \text { in (I.): }=(\mu+1)^{2}=\frac{1}{4}(m+2)^{2}, \\
& \text { in (II.): }=v(\nu+1)=\frac{1}{4}(m+1)(m+3) .
\end{aligned}
$$

Daraus ist zu entnehmen, durch wieviele gegebene Puncte $p$ eine Curve $C^{m}$ bestimmt wird, wenn sie durch dieselben gehen und einen andern gegebenen Punct $\mathfrak{M}$ zum Mittelpunct haben soll. 
10 2. Stein er, ulgeb. Curven, welche Mittelp. haben, u. Eigens. allgem. algeb. Curven.

selben Punct $\mathfrak{M}$ gehälftet werden, so liegen ihre $2 \mu(\mu+2)$ oder $2 \nu(\nu+1)-2$ Endpuncte $p$ und $p_{1}$ allemal in einer durch sie bestimmten Curve $C^{2 \mu}$ oder $C^{2 v^{-1}}$, welche den Punct $\mathfrak{M}$ sum Mittelpunct hat."

§. 3.

Läfst man von den genannten Sehnen $p p_{1}$ eine weg, so ist die Curve durch die Endpuncle der übrigen nicht mehr bestimmt, aber durch jeden Punct $p^{\prime \prime}$, den man frei annimmt und durch den sie gehen soll, wird sie bestimmt (weil dann nebst $\mathfrak{M}$ wieder eben soviele $p$ gegeben sind, wie vorhin), so dafs also unendlich viele Curven $\boldsymbol{C}^{m}$ durch diese übrigen Endpuncte möglich sind, die $\mathfrak{M}$ zum Mittelpunct haben. Aber alle diese Curven schneiden einander, aufser den Endpuncten der Sehnen, noch in anderen bestimmten Puncten $q$ und $y_{1}$, deren Zahl beziehlich $2(\mu-1)^{2}$ und $2(v-1)(v-2)+1$ ist, so dafs sie ein Curvenbüschel $\boldsymbol{B}\left(\boldsymbol{C}^{\prime m}\right)$ mit $\boldsymbol{m}^{2}$ Grundpuncten bilden (s. den obigen Monatsbericht). Die neuen Puncte sind eben so paarweise die Endpuncte von Sehnen $q q_{1}$, welche ihre Mitlen in $\mathfrak{M}$ haben; und im zweiten Falle, wo $m=2 v-1$, liegt der ungerade oder einzelne Punct, etwa $q_{0}$, in $\mathfrak{M}$ selbst. Also:

,Sind $\mu(\mu+2)-1$ oder $\nu(\nu+1)-2$ beliebige Sehnen pp gegeben, die alle durch denselben Punct $\mathfrak{M}$ gehälfiel uerden, so gehen durch ihre Endpuncte ein Curvenbüschel $\boldsymbol{B}\left(C^{2 \mu}\right)$ oder $\boldsymbol{B}\left(C^{2 \nu-1}\right)$, welche alle den Punct $\mathfrak{M}$ zunn Mittelpunct huben, und deren übrige $2(\mu-1)^{2}$ oder $2(\nu-1)(\nu-2)+1$ gemeinschuflliche Schnittpuncle ( $q$ und $\left.q_{1}\right)$ ebenfalls paarweise die Endpuncte solcher Sẹhnen $q q_{1}$ sind, die ihre Mitten in $\mathfrak{M}$ haben. Im zweiten Falle liegt der, einzelne Punct $y_{0}$ in Mittelpuncte $\mathfrak{M}$ selbst, so da/s alle Curven des Büschels $\boldsymbol{B}\left(C^{2 v-1}\right)$ durch ihren gemeinsumen Mittelpunct gehen, der zugleich ein Wendepunct von jeder ist."

So gehen also z. B. durch die vier Endpuncte zweier Sehnen $p p_{1}$ ein Kegelschnitt-Büschel $\boldsymbol{B}\left(\boldsymbol{C}^{2}\right)$, welche alle $\mathfrak{M}$ zum Mittelpunct, die beiden Sehnen zu Durchmessern, aber weiter keinen Punct gemein haben, weil $2(\mu-1)^{2}=0$, wenn $\mu=1$ ist. Durch die 8 Endpuncte von 4 Sehnen $p p_{1}$ gehen ein $B\left(C^{j}\right)$, die noch einen $9^{\text {ten }}$ Punct $q_{10}$ gemein haben müssen, welcher der Mittelpunct $\mathfrak{M}$ selbst und zugleich Wendepunct von jeder ist. Durch die 14 Endpuncte von 7 Sehnen $p p_{1}$ gehen ein $\boldsymbol{B}\left(C^{4}\right)$, welche $\mathfrak{M}$ zum Mitlelpunct haben und sich noch in den Endpuncten einer neuen Sehne $q q_{1}$ schneiden, die gleichfalls ihre Mitte in $\mathfrak{M}$ hat; u. s. w.

Einige andere Eigenschaften der obigen Curvenbüschel treten weiter unten gelegentlich hervor. 
§. 4.

Zum Behuf späterer Betrachtungen mag hier bemerkt werden, dafs eine Curve $C^{m}$, welche einen Mittelpunct hat, auch in solcher specieller Form erscheinen kann, wo sie aus verschiedenen Theilen besteht. So kann z. B. der Kegelschnitt $C^{2}$

1) Durch zuei sich schneidende Gerade $A$ und $B$ vertreten werden, deren Schnittpunct als Mittelpunct $\mathfrak{M}$ anzusehen ist; oder

2) Durch zwei parallele Gerade, $\boldsymbol{A}$ \# $\boldsymbol{B}$, wo danı der Millelpunct unbestimnt bleibt, nümlich jeder Punct sein kann, welcher von $A$ und $B$ gleichweit absteht, also eine dritte Gerade $C$ zum Ort hat, die mit $A$ und $B$ parallel und in der Mitte swischen ihnen liegt.

Gleicherweise kann eine Curve $\boldsymbol{C}^{3}$, welche einen Mittelpunct haben soll, insbesondere durch folgende Elemente vertreten werden.

1) Durch einen Kegelschnitt. $C^{2}$ und irgend eine durch seinen Mitlelpunct gehende Gerade $C^{1}$, wohei der Mittelpunct $\mathfrak{M}$ von $C^{2}$ auch zugleich als Miltelpunct von $C^{3}\left(=C^{2}+C^{1}\right)$ anzusehen ist. (Gilt also auch, wenn $C^{2}$ eine Parabel und $C^{1}$ irgend ein Durchmesser derselben ist.)

2) Durch drei Gerude und zwar a) durch drei sich in einem Punct schneidende Gerade, wo dann dieser Punct selbst der Mittelpunct ist (hierin sind auch die zwei besondern Zustände inbegriffen, wo die drei Geraden parallel, oder zwei parallel und die dritte im Unendlichen); oder b) durch zwei parallele und eine sie schneidendé Gerade, wobei die Mitte des von jenen beiden auf der letstern begrensten Stücks der Mittelpunct ist; oder endlich c) durch drei parullele Gerade, wenn die eine gleich weil von den beiden andern absteht, wobei dann jeder Punct in der mittlern Geraden als Mittelpunct anzusehen ist.

Analogerweise kann die Curve $C^{4}$ in Theile zerfallen; u. s. w.

\$. 5.

Die obige zweite Frage (\$. 2.) verlangt zu wissen: „Wieviele beliebige Puncte $p$ dürfen höchstens gegeben uerden, wenn durch dieselben eine Curve $C^{m}$ gehen soll, welche einen Mittelpunct hat, der aber nicht gegeben ist."

Man überzeugt sich leicht, dafs unter dieser Bedingung nur zwei Puncte $p$ mehr gegeben werden dürfen, als im obigen Falle (\$. 2.), wo der Mittelpunct $\mathfrak{M}$ selbst mit gegeben war. Denn sobald nur ein Punct, etwa q, mehr 
12 2. Steiner, algeb. Curven, welche Mittelp. haben, u. Eigens. allgem. algeb. Curven.

gegebèn, so kann $\mathfrak{M}$ schon nicht mehr beliebig liegen, sondern mufs sich auf einen Ort beschränken, der irgend eine Curve $\mathfrak{M}^{x}$ sein wird; und wenn man statt $q$ einen andern beliebigen Punct $r$ als gegeben annimmt, so wird der Mittelpunct $\mathfrak{M}$ der Curve $C^{m}$ einen andern Ort, etwa $\mathfrak{M}_{1}^{x}$, haben; und soll nun eine Curve $C^{m}$ durch beide Puncte $q$ und $r$ gehen, so kann ihr Mittelpunct $\mathfrak{M}$ nur in einem den Ortscurven $\mathfrak{M}^{x}$ und $\mathfrak{M}_{1}^{x}$ gemeinsamen Puncte liegen. Da diese Ortscurven sich aber in mehreren Puncten schneiden, so wird die Curve $C^{m}$ nicht absolut bestimmt sein, sondern die gestellten Bedingungen werden mehrere Lösungen gestatten. Also:

„Soll eine Curve $C^{m}$ einen Mitfelpunct haben, so ist sie

$\alpha)$ als $C^{2 \mu}$ durch $\mu(\mu+2)+2=\frac{1}{4}[m(m+4)+8]$,

(3) als $C^{2 v-1}$ durch $\nu(\nu+1)+1=\frac{1}{4}[\operatorname{mon}(m)+7]$

beliebig gegebene Puncte p bestimmt, jedoch nicht absolut bestimm/, sondern es finden im Allgemeinen mehrere Lösungen statt."

Wie es sich damit näher verhält, ist aus den nachfolgenden zwei einfachsten Beispielen zu ersehen.

\$. 6 .

Erstes Beispiel. Soll ein Kegelschnitt $C^{2}$ durch 4 gegebene Puncte $3 p$ und $q$ gehen, so ist der Ort seines Mittelpuncts $\mathfrak{M}$ ein bestimmter anderer Kegelschnitt $\mathfrak{M}^{2}$; und soll $C^{2}$ durch die $3 p$ und einen anderen gegebenen Punct $r$ gehen, so ist der Ort seines Mittelpuncts ein neuer Kegelschnitt $\mathfrak{M}_{1}^{2}$. Nun schneiden sich die beiden Örter $\mathfrak{M}^{2}$ und $\mathfrak{M}_{1}^{2}$ zwar in 4 Puncten: aber von, diesen 4 Puncten besitzt nur einer die Eigenschaft, dafs er der Mittelpunct $\mathfrak{M}$ eines Kegelschnitts $C^{2}$ ist, welcher durch die 5 Puncte $3 p, q$ und $r$ geht; die drei übrigen haben diese Eigenschaft nicht, denn sie sind die Mitten der Seiten desjenigen Dreiecks, dessen Ecken die $3 p$ sind, und bängen somit von diesen $3 p$ allein ab. Nämlich bezeichnet man die $3 p$ durch $a, b$, c, so geht bekanntlich der genannte Kegelschnitt $\mathfrak{M}^{2}$ durch die Mitten der 6 Seiten des vollständigen Vierecks abcq; und eben so geht $\mathfrak{M}_{1}^{2}$ durch die Mitten der 6 Seiten des vollständigen Vierecks abcr: somit gehen beide durch die Mitten der 3 Seiten des Dreiecks abc, aber jede dieser 3 Mitten ist Mittelpunct zweier verschiedener Kegelschnitte $\boldsymbol{C}^{2}$, wovon der eine dem Viereck abcq, der andere dem Viereck abcr umschrieben ist.

S. 7.

Zweites Beispiel. I. Die Curve $C^{3}$ ist durch den gegebenen Miltelpunct $\mathfrak{M}$ und durch 5 Puncte $p$, durch welche sie gehen soll, bestimmt; ist 
2. St eiver, algeb. Curven, welche Mittelp. haben, u. Eigens. allgem. algeb. Curven. 13

nun die Lage von $\mathfrak{M}$ nicht gegeben, aber dagegen noch ein $6^{\text {ter }}$ Punct $q$, durch welchen $C^{3}$ gehen soll, so findet folgender Satz statt:

„Soll eine Curve dritten Grads, $C^{3}$, durch gegebene 6 Puncte $5 p$ und $q$ gehen, und einen Mittelpunct $\mathfrak{M}$ haben, so ist der Ort des letzlern eine Curve $\overline{5}^{\text {ten }}$ Grads, $=\mathfrak{M}^{5} . "$

Von dieser Ortscurve $\mathfrak{M}^{5}$ sind nachstehende 57 Puncte theils unmittelbar gegeben, theils leicht zu construiren, indem sie die Mittelpuncte specieller Curven $\boldsymbol{C}^{3}$ sind. Nämlich die Curve $\mathfrak{M}^{5}$ geht:

1) Durch die gegebenen 6 Puncte selbst; denn jeden derselben kann man als $\mathfrak{M}$ annehmen und verlangen, die Curve $C^{3}$ soll durch die 5 übrigen gehen (\$. 2.).

2) Durch die Mitten $\mu$ der 15 Geraden $G$, welche die gegebenen 6 Puncte parweise verbinden; denn man kann die Mitte $\mu$ einer solchen Geraden $G$ als $\mathfrak{M}$ annehmen und verlangen, die $C^{3}$ soll durch den einen Endpunct von $G$ und durch die 4 übrigen gegebenen Puncte gehen; so geht sie auch zugleich durch den andern Endpunct von $\boldsymbol{G}$.

3) Durch die Mittelpuncte $m$ der 6 Kegelschnitte $C^{2}$, welche einzeln durch je 5 der gegebenen 6 Puncte gehen. Denn ein solcher $C^{2}$ und sein durch den $6^{\text {ten }}$ Punct gehender Durchmesser sind zusammen eine specielle $\boldsymbol{C}^{3}$, welche mit $\boldsymbol{C}^{2}$ den Mittelpunct gemein hat (\$. 4.).

4) Durch die Mittelpuncte $m_{1}$ der 30 Kegelschnitte $C_{1}^{2}$, wovon jeder einzeln durch 4 der gegebenen 6 Puncte geht und seinen Mittelpunct in der die 2 übrigen verbindenden Geraden $G$ hat. Denn ein solcher $C_{1}^{2}$ und die zugehörige $\boldsymbol{G}$ sind zusammen eine $C^{3}$, welche durch alle 6 Puncte geht und mit $C_{1}^{2}$ denselben Mittelpunct hat. In jeder Geraden $G$ liegen 2 Mittelpuncte $m_{1}$.

Dies sind zusammen 57 Puncte: 1) $5 p+q$; 2) $15 \mu$; 3) $6 m$; und 4) $30 m_{1}$.

In jeder der 15 Geraden $G$ kennt man demnach alle ihre 5 Schnitte mit der Curve $\mathfrak{M}^{5}$ : nämlich ihre zwei Endpuncte $(2 p$, oder $p$ und $q)$, ihre Mitte $\mu$ und die in ihr liegenden $2 m_{1}$.

Um die Bestimmung der 30 Mittelpuncte $m_{1}$ deutlicher zu machen, bezeichne man die $5 p$ durch $a, b, c, d, e$. Je 4 der gegebenen 6 Puncte, etwa $a, b, c$ und $d$, bestimmen $6 G$, deren Mitten, $6 \mu$, in einem Kegelschnitte $\mathfrak{M}^{2}$ liegen, welcher der Ort der Mittelpuncte aller durch $a, b, c$ und $d$ gehenden Kegelschnitte $\left(C_{1}^{2}\right)$ ist (\$.6.), und welcher somit die durch $e$ und $q$ 
14 2. St ein er, alyeb. Curven, welche Mittelp. haben, u. Eigens. allgem. algeb. Curven.

gehende $G$ in den genannten $2 m_{1}$ schneidet; ferner geht $\mathfrak{M}^{2}$ auch durch die Mittelpuncte, $2 m$, der beiden Kegelschnitte $C^{2}$, welche beziehlich durch die 5 Puncte abcde und abcdq bestimmt werden (3); folglich kennt man auch alle Schnitte des Kegelschnitts $\mathfrak{M}^{2}$ mit der Curve $\mathfrak{M}^{5}$, nämlich die genannten $6 \mu, 2 m_{1}$ und $2 m$, zusammen $=10$ Schnitle. Es giebt im Ganzen 15 solche Kegelschnitte $\mathfrak{M}^{2}$.

II. Durch das Vorstehende (I.) läfst sich nunmehr auch leicht entscheiden, wieviele Curven $C^{3}$, welche Mittelpuncte haben, durch 7 gegebene Puncte $5 p, q$ und $r$ gehen. Denn soll die $C^{3}$ nur durch die 6 Puncte $5 p$ und $r$ gehen, so ist gleicherweise, wie vorhin (I.), der Ort ihres Mittelpuncts $\mathfrak{M}$ eine neue Curve $\mathfrak{M}_{1}^{5}$; und soll also $C^{3}$ durch alle 7 Puncte zumal gehen, so mufs ihr Mittelpunct in beiden Ortscurven $\mathfrak{M}^{5}$ und $\mathfrak{M}_{1}^{5}$ zugleich liegen, d. h. er mufs einer ihrer gegenseitigen Schnitte sein. Nun ist die Zabl dieser Schnitte $=25$; allein nach der obigen Auseinandersetzung befinden sich darunter 16 solche, welche der Forderung nicht genügen können, weil sie von den $5 p$ allein abhängen, nämlich dieselben sind 1) die $5 p$ selbst, 2) $10 \mu$, d. h. die Mitten der durch die $5 p$ bestimmten 10 Geraden $G$, und 3 ) ein $m$, der Mittelpunct des durch die $5 p$ gehenden Kegelschnitts $C^{2}$; denn durch diese 16 Puncte gehen beide Ortscurven; daher bleiben für die Lage des Mittelpuncts $\mathfrak{M}$ der Curve $C^{3}$ nur 9 Schnittpuncte übrig. Dies begründet den folgenden Satz:

„Durch 7 gegebene Puncte in einer Ebene gehen, im Allyemeinen, nur 9 solche Curven dritten Grads, welche Mittelpuncte haben."

Daraus schliefst man: a) Da/s unter den unendlich vielen Curven dritten Grads $A^{3}$, welche durch beliebig gegebene 8 Puncte gehen, und somit einen Curvenbüschel $B\left(A^{3}\right)$ mit 9 gemeinschaftlichen Puncten bilden, sich in Allgemeinen keine befindet, welche einen Mittelpunct hat. b) Hat aber insbesondere eine der Curven einen Mittelpunct, so braucht deshalb von den ubrigen keine einen Mitlelpunct zu haben. c) Befinden sich insbesondere zwei darunter, welche Mittelpuncte haben, aber micht concentrisch sind, so kann von den übrigen keive einen Mitlelpunct habew, d. h. "durch die Schnittpuncte zweier Curven $A^{3}$, welche Mittelpuncte haben, aber micht concentrisch sind, kann keine dritte geken, welche ebenfalls einen Mittelpunct hat." d) Wei/s wan von drei Curven $A^{3}$, do/s sie 8 Puncte gemein haben und dafs jede einen Mittelpunct hat: so folgt, dafs sie concentrisch sein müssen, und du/s alle zu ihrem Büschet ge- 
2. St einer, algeb. Curven, welche Mittelp. haben, u. Eigens. allgem. algeb. Curven. 15

hörigen Curven ebenfalls Mittelpuncte haben und mit ihnen concentrisch sind, und da/s jene 8 (oder 9) Puncte die oben (\$. 3.) beschriebene besondere Lage haben müssen. - Analoges findet bei den höheren Curven statt.

5. 8.

In Betracht der Ortscurve $\mathfrak{M}^{5}$ (\$. 7. I.) sind durch besondere Wahl der gegebenen 6 Puncte, $5 p$ und $q$, oder $a, b, c, d$, $e$ und $q$, zahlreiche specielle Fälle möglich, von denen einige hier kurz angedeutel werden sollen.

I. Wenn die gegebenen 6 Puncte in einem Kegelschnitte $C_{0}^{2}$ liegen, dessen Mittelpunct $\mathfrak{M}_{0}$ hei/sen mag: so vereinigen sich die dort genannten 6 Kegelschnitte $C^{2}$ (\$. 7. I. 3.) alle in $C_{0}^{2}$ und ihre sechs Miltelpuncte $m$ in $\mathfrak{M}_{0}$. Da $C_{0}^{2}$ mit jedem seiner Durchmesser $C_{0}^{1}$ zusammen eine $C^{3}$ vorstellt, welche durch die 6 Puncte geht und $\mathfrak{M}_{0}$ zum Mittelpunct hat: so folgt da/s $\mathfrak{M}_{0}$ ein vielfacher Punct der Curve $\mathfrak{M}^{5}$ sein mu/s. - Oder, wenn der durch die 5 Puncte $a, b, c, d, e$ gehende Kegelschnitt $C^{2}$ den $6^{\text {ten }}$ Punct $q$ zum Mittelpunct hat, so folgt eben so, dafs dunn die Curve $\mathfrak{M}^{5}$ den Punct $q$ zum Doppelpunct haben mu/s.

II. Liegen von den 6 Puncten drei, etwa $d$, $e$ und $q$, in einer Geraden B: so mu/s $\mathfrak{M}^{3}$ in diese Gerade und in eine Curve $\mathfrak{M}^{4}$ zerfallen, so da/s $\mathfrak{M}^{5}=\boldsymbol{B}+\mathfrak{M}^{4}$. Denn jeder beliebige Punct $\mathfrak{N}$ in der Geraden $\boldsymbol{B}$ ist Miltelpunct eines Kegelschnitts $\mathfrak{N}^{2}$ der durch die 3 Puncte $a, b, c$ geht, und der also mit $\boldsymbol{B}$ zusammen eine Curve $\boldsymbol{C}^{3}$ repräsentirt, welche durch die 6 Puncte geht und ihren Mittelpunct $\mathfrak{M}$ in $\mathfrak{N}$ hat; so dafs folglich $\boldsymbol{B}$ zum Ort der Mittelpuncte $\mathfrak{M}$ gehört. - Die Curve $\mathfrak{M}^{4}$ geht durch folgende leicht angebbare 39 Puncte. 1) Durch $a, b$ und $c$; 2) durch die Mitten $\mu$ sowohl der $3 G$, welche die Puncte $a, b, c$ unter sich, als der 9G, welche $a, b, c$ mit $d ; \rho, q$ verbinden, also durch $12 \mu ; 3)$ durch die Mittelpuncte on der $3 C^{2}$, welche beziehlich durch die dreimal 5 Puncte abcde, abcdq, abceq gehen; 4) durch 18 Puncte $m_{1}$, in welchen die. vorgenannten $9 G$ von den ihnen (wie oben $\$$. 7. I.) entsprechenden Kegelschnitten $\mathfrak{M}^{2}$ geschnitten werden; und ferner durch 3 Puncte $m_{1}$, in welchen die vorgenannten $3 G$, d. i. $a b, a c, b c$ beziehlich von 3 Geraden $C_{1}, B_{1}, A_{1}$ geschnitten werden, die so bestimmt sind, dafs z. B. $C_{1}$ durch die Milten $\mu$ der 3 Geraden $c d$, ce und $c q$ geht und die $a b$ in $m_{1}$ trifft. Demnach kennt man die 4 Schnitte von jeder der 15 Geraden $3 G, 9 G, A_{1}, B_{1}$ und $C_{1}$ mit der Curve $\mathfrak{M}^{4}$; eben so die 8 Schnitte von jedem der 9 Kegelschnitte $\mathfrak{M}^{2}$ mit $\mathfrak{M}^{4}$. 
16 2. Steiner, algeb. Curven, welche Mittelp. haben, u. Eigens. allgem. algeb. Curven.

III. Liegen die 6 Puncte $z$ u und 3 in swei Geraden, etwa $\iota, b, c$ in $A$, und $d, e, q$ in $B:$ so mu/s die Ortscurve $\mathfrak{M}^{5}$ aus diesen Geraden, und aus einer Curve $\mathfrak{M}^{3}$ bestehen, so da/s $\mathfrak{M}^{5}=A+B+\mathfrak{M}^{3}$. Die Curve $\mathfrak{M}^{3}$ geht durch folgende, leicht construirbare, 27 Puncte. 1) Durch $9 \mu$, die Mitten der $9 G$, welche die Puncte in $\boldsymbol{A}$ mit denen in $\boldsymbol{B}$ verbinden; 2) durch die $18 n_{1}$, in welchen die $9 G$ von den zugehörigen $9 \mathfrak{M}^{2}$ geschnitten werden. Somit kennt man die 3 Schnitte jeder der $9 G$ mit $\mathfrak{M}^{3}$. Jene $9 \mu$ liegen auch zu 3 und 3 in 6 Geraden, $3 A_{1}$ und $3 B_{1}$, wovon die $3 A_{1}$ mit $A$, und die $3 B_{1}$ mit $B$ parallel sind.

IV. Gehen von den $15 G$, welche die 6 Puncte paarweise verbinden, irgend $3 G$, die zusammen alle 6 Puncte enthalten, etwa die 3 Geraden $a b, c d$ und $e q$, durch irgend einen Punct $N$, so vertreten sie eine $C^{3}$, deren Mittelpunct $\mathfrak{M}$ in $\boldsymbol{N}$ liegt (\$. 4.). Sind insbesondere die 3 Geraden $a b, c d$, eq parallel und liegt $\boldsymbol{c} d$ in der Mitte zwischen den beiden andern: so zerfällt $\mathfrak{M}^{5}$ in die Gerade $c d$ und in eine Curve $\mathfrak{M}^{4}$, von der 46 Puncle leicht anzugeben sind, nämlich aufser $a, b, e, q$ noch $10 \mu, 6 m$ und $26 m_{1}$. Sind zum zweiten Mal drei Gerade parallel und die miltlere gleichweit von den äufsern entfernt, welche jedoch nur (wenn man sich bei jenen erstern $a b, c d$, eq die Endpuncte $a, c, e$ nach links und $b, d, q$ nach rechts denkt) entweder $\alpha$ ) die Geraden $a c, b e, d q$ oder $(\beta) a e, c q, b d$ sein können: so müssen nothwendig zum dritten Mal 3 Gerade dieselbe Eigenschaft haben, und zwar beziehlich ( $\alpha) b d$, $a q$, ce oder $(\beta) b c$, be, ce. In beiden Fällen schneiden sich die 3 mittleren Geraden $c d$, be, ay oder $c d, c y, b e$ in einem und demselben Puncte $\boldsymbol{N}_{0}$; aber im Falle $(\alpha)$ sind sie die Hauptdiagonalen eines Sechsecks abdyeca, welches die 3 Paar äufseren Geraden zu Gegenseiten hat, wogegen im Falle $(\beta)$ die 3 Geraden des dritten Systems, $b c$, be, ce in eine und dieselbe Gerade, bce, fallen und wobei $N_{0}$ in $c$ liegt. Für beide Figuren besteht $\mathfrak{M}^{5}$ aus den drei mittlern Geraden, $c d, b e, a q$ oder $c d, c q, b e$, und aus einem Kegelschnitte $\mathfrak{M}^{2}$, welcher bei der ersten Figur die Seiten des genannten Sechsecks in ihren Mitten berührt und $\boldsymbol{N}_{0}$ zum Mittelpunct hat; etc. Die 6 Puncte können endlich auch solche specielle Lage haben, dafs von den $15 G$ sich $10 \mathrm{mal} 3 G$, die zusammen alle 6 Puncte enthalten, in einem Puncte $\boldsymbol{N}$ treffen, wobei dann $\mathfrak{M}^{5}$ in 5 Gerade $\mathfrak{M}^{1}$ zerfällt. Die einfachste Figur, diesen Fall darzustellen, ist die, wo etwa $u, b, c, d, e$ die Ecken eines regelmäfsigen Fünfecks sind und $y$ der Miltelpunct des demselben umschriebenen Kreises. Die 5 Geraden $\mathfrak{M}^{1}$ sind alsdann $q a, q b, q c$, qd und qe; 
die 10 Puncte $\boldsymbol{N}$ liegen paarweise in ihnen und sind, zu 5 und 5, die Ecken zweier neuen regelmäfsigen Fünfecke, welche gleichfalls $q$ zum Centrum haben. In diesem Falle ist jedoch keine eigentliche Curve $C^{3}$ mehr möglich, sondern jede besteht aus $C^{2}+C^{1}$, und zwar ist $C^{1}$ immer diejenige von den 5 Geraden $\mathfrak{M}^{1}$, in welcher der Mittelpunct $\mathfrak{M}$ von $C^{2}$ liegt. Liegt $\mathfrak{M}$ insbesondere in einem der $10 \mathrm{~N}$, so besteht $C^{3}$ aus 3 Geraden, $=3 C^{\mathbf{1}}$.

S. 9.

Die Curven, welche Mittelpuncte haben, besitzen, in Bezug auf dieselben, verschiedene wesentliche Eigenschaften, wovon einige hier näher angegeben werden sollen.

Zur Abkürzung soll dabei, so wie in der Folge ein Doppelpunct durch $d p$ oder $\mathfrak{P}_{2}$, eine Doppeltangente durch $d t$ oder $\mathfrak{I}_{2}$, ein Wendepunct durch $w p$ oder $\mathfrak{w}$, eine Wendetangente durch wt oder $\mathfrak{W}$, ein Rückkehrpunct durch $\boldsymbol{r p}$ oder $\mathfrak{r}$, eine Rückkehrtangente durch $r t$ oder $\Re$, eine Asymptote durch $\boldsymbol{A}_{s}$ und die unendlich entfernte Gerade der Ebene durch $\boldsymbol{G}_{\infty}$ bezeichnet werden.

I. Hat eine Curve $C^{m}$ einen Mittelpunct $\mathfrak{M}$, so gehen ihre m Asymptoten $\boldsymbol{A}_{s}$, im Allgemeinen, alle durch denselben. Jede andere durch den Mittelpunct gehende Tangente der Curve ist nothwendig eine Doppeltangente $\mathfrak{I}_{2}$, und ihre swei Berührungspuncte, etwa $b$ und $b_{1}$, sind Gegenpuncte. Die Zahl der durch $\mathfrak{M}$ gehenden $\mathfrak{I}_{2}$ ist $=\frac{1}{2} m(m-2)$, und ihre $m(m-2)$ Berührungspuncte, $b$ und $b_{1}$, liegen in einer neuen Curve $C^{m-2}$, welche ebenfalls einen Mitlelpunct, und zuar mit der gegebenen den nämlichen Punct $\mathfrak{M}$ sum Mittelpunct hat. Von dieser neuen Curve gehen also eben so alle, $\boldsymbol{A}_{s}$ so wie eine ihrem Grad angemessene Zahl $\mathfrak{I}_{2}$ durch den Mittelpunct $\mathfrak{M}$, und die Berührungspuncte der $\mathfrak{T}_{2}$ liegen in einer neuen Curve $C^{m-4}$, uelche gleicherweise denselben Punct $\mathfrak{M}$ zum Mittelpunct hat; u. s. w. Werden die zwei Zahlformen von $m$ unterschieden, so entstehen auf diese Weise zwei Curvenreihen:
а) $C^{12 \mu}, C^{2 \mu-2}, C^{2 \mu-4}, \ldots, C^{4}, C^{2}$;
B) $C^{2 \nu-1}, C^{2 \nu-3}, C^{2 \nu-5}, \ldots, C^{3}, C^{1}$.

Crelle's Journal f. d. M. Bd. XLVII, Heft 1. 
18 2. St einer, algeb. Curven, welche Mittelp. haben, u. Eigens. allgem. algeb. Curven.

Bei $(\alpha)$ hat die vorletzte Curve, $C^{4}$, noch $4 \mathfrak{I}_{2}$ mit 8 Berührungspuncten, durch welche die letzte, $C^{2}$, geht; und diese $C^{2}$ hat nur noch $2 A_{s}$, aber keine $\mathfrak{I}_{2}$ mehr. Da für $C^{2 \nu-1}$ die Zahl der durch ihren Mittelpunct gehenden $\mathfrak{I}_{2}=2 \boldsymbol{v}(\nu-2)+\frac{3}{2}$ ist, so hat das vorletzte Glied bei $(\beta), C^{3}$, nur $\frac{3}{2} \mathfrak{I}_{2}$, was offenbar ihre Wendetangente im Mittelpuncte $\mathfrak{M}$ bedeutet, und das letzte Glied $C^{\mathbf{1}}$ ist diese $w t$ selbst. Übrigens haben alle Curven der Reihe $(\beta)$ diese nämliche $C^{1}$ zur gemeinschaftlichen $w t$, so dafs dieselben in ihrem gemeinsamen Mittel- und Wendepunct $\mathfrak{M}$ sich insgesammt dreipunctig berühren. Auch für die Curve $C^{2 v-1}$ bedeutet der Bruch, $\frac{3}{2}$, die Wendetangente im Panct $\mathfrak{M}$ selbst, und die Zahl der eigentlichen Doppeltangenten ist $=2 \nu(\nu-2)$.

II. Die Tangenten in je swei Gegenpuncten $p$ und $p_{1}$ der Curve $C^{m}$ sind parallel. Alle ausgezeichneten Elemente der Curve, als da sind $d p, w p, r p, d t$, wt und rt, wofern sie nicht im Mittelpunct $\mathfrak{M}$ oder im Unendlichen, in $G_{\infty}$ liegen, müssen paaruseise vorhanden und swar Gegenelemente sein. $D$. h. die $3 m(m-2)$ w der Curve müssen paurweise Gegenpuncle, und die jedem Paar zugehörigen $\mathfrak{W}$ müssen parallel sein; die $\frac{1}{2} m(m-2)\left(m^{2}-10\right) \mathfrak{I}_{2}$, die nicht durch den Mittelpunct $\mathfrak{M}$ gehen, müssen paarueise parallel sein und gleich weit von $\mathfrak{M}$ abstehen, auch sind die Berührungspuncte jedes Paars beziehlich Gegenpuncte; hat die Curve Doppelpuncte, $\mathfrak{p}_{2}$, (die ureder in $\mathfrak{M}$ noch in $G_{\infty}$ liegen), so müssen dieselben paarweise vorhanden und Gegenpuncte sein, auch müssen die zwei Tangenten in dem einen $\mathfrak{p}_{2}$, denen in seinem Gegenpuncte beziehlich parallel sein; eben so können auch die Rückkehrpuncte $\mathfrak{r}$ nur paarweise und zwar als Gegenpuncte auftreten, und die sugehörigen Rükkehrtangenten müssen parallel sein. Hat dagegen die Curve einen Doppelpunct, der insbesondere im Unendlichen, in $G_{\infty}$, (oder in $\left.\mathfrak{M}\right)$ liegt, so bedingt derselbe nicht gleicherueise einen zweiten, vielmehr beuirkt er ungekehrt sogar noch eine scheinbare Abweichung von dem obigen Satze (I.). Nümlich, liegt ein Doppelpunct in $G_{\infty}$, so erscheinen die beiden Tangenten in demselben als zwei parallele Asymptoten, die, jenem Satze entgegen, nicht durch den Mittelpunct $\mathfrak{M}$ gehen, wohl aber gleichweit von'M abstehen; daher kann $G_{\infty}$. selbst nie Tangente der Curve in einem Doppelpuncte sein. Und liegt ferner ein Rükkehrpunct in $\boldsymbol{G}_{\infty}$, so mu/s die Rückkehrtangente entweder auf $G_{\infty}$ fallen oder durch $\mathfrak{B}$ gehen, wo sie dann im letztern Falle als zweifache (oder im weitern Sinne als fünffache) Asymptote anzusehen ist. 
III. Zieht man durch den Mittelpunct $\mathfrak{M}$ der Curve $\boldsymbol{C}^{m}$ irgend eine unbegrenzte Gerade, einen Durchmesser $\boldsymbol{S}$, so liegen in ihm $\frac{1}{2} m$ Paare Gegenpuncte $q$ und $q_{1}$, oder $\frac{1}{2} m$ Sehnen $q q_{1}$, und die Tangenten in jedem dieser Punctenpare sind parallel, und zwar hat jedes Tangentenpaar, im Allgemeinen, eine besondere Richtung, so dafs, wenn man diese Richtungen der Tangenten, wie beim Kegelschnitt, dem Durchmesser $\boldsymbol{S}$ (oder den respectiven Sehnen $\boldsymbol{q} \boldsymbol{q}_{1}$ ) ,conjugirt" nennen wollte, alsdann zu demselben Durchmesser $\frac{1}{2} m$ verschiedene conjugirte Richtungen gehörten. Eben so würden umgekehrt zu jeder bestimmten Richtung der Tangenten auch mehrere conjugirte Durchmesser gehören; denn nach jeder gegebenen Richtung $\boldsymbol{R}$, d. h. mit irgend einer gegebenen Geraden $\boldsymbol{R}$ parallel, sind im Allgemeinen $\boldsymbol{m}(\boldsymbol{m}-1)$ Tangenten möglich, deren Berührungspuncte nothwendig paarweise Gegenpuncte oder Endpuncte von Sehnen $q q_{1}$ sein müssen, so dafs also einer und derselben Richtung $\boldsymbol{R}$, in dieser Hinsicht, $\frac{1}{2} m(m-1)$ verschiedene Durchmesser $\boldsymbol{S}$ (oder Sehnen $4 q_{1}$ ) conjugirt sind. In diesem Sinne kann man also sagen: „Zu jedem Durchmesser $\boldsymbol{S}$ gehören $\frac{1}{2}$ m conjugirle Richtungen $R$, und zu jeder Richtung $\boldsymbol{R}$ gehören $\frac{1}{2} m(m-1)$ conjugirte Durchmesser $\boldsymbol{S}$ oder Sehnen $q q_{1} . "$ *)

Nun liegen ferner die $m(m-1)$ Berührungspuncte jedes Systems paralleler Tangenten bekanntlich in einer neuen Curve $C^{m-1}$, welche die erste Polare des nach der Richtung der Tangenten im Unendlichen, in $\boldsymbol{G}_{\infty}$, gedachten Pols $\boldsymbol{P}_{\infty}$ heifst; und da die Berührungspuncte paarweise Gegenpuncte, oder die Endpuncte von $\frac{1}{2} m(m-1)$ Sehnen $q y_{1}$ sind, so mufs diese Curve ebenfalls den Punct $\mathfrak{M}$ zum Mittelpunct haben. Gleicherweise müssen die $2^{\text {te }}, 3^{\text {te }}, \ldots,(m-1)^{\text {ste }}$ Polare desselben Pols $\boldsymbol{P}_{\infty}$ in Bezug auf die gegebene Curve $\boldsymbol{C}^{m}$, welche nach der Reihe $\boldsymbol{C}^{m-2}, \boldsymbol{C}^{m-3}, \ldots \boldsymbol{C}^{2}, \boldsymbol{C}^{1}$ sind, den nämlichen Punct $\mathfrak{M}$ zum Mittelpunct haben, wobei die letzte, $C^{1}$, eine durch $\mathfrak{M}$ gehende Gerade, ein Durchmesser von jeder der übrigen Polaren, so wie von $C^{m}$ ist. Also:

„Hat eine Curve $C^{\prime m}$ einen Mittelpunct $\mathfrak{M}$, so haben auch alle successiven Polaren $\mathrm{C}^{m-1}, \mathrm{C}^{m-2}, \mathrm{C}^{m-3}, \ldots \boldsymbol{C}^{2}, \boldsymbol{C}^{1}$ jedes unendlich entfernten Pols $\boldsymbol{P}_{\infty}$ Mittelpuncte, und swar sind sie alle mil der $\boldsymbol{B a s i s} \boldsymbol{C}^{m}$ concentrisch."

*) Hierbei entsteht die doppelte Frage:

,Welche Relation findet einerseits zwischen den $\frac{1}{2} m$ conjugirten Richtungen $\boldsymbol{R}$ zu jedem Durchmesser $S$; und andererseits zwischen den $\frac{1}{2} m(m-1)$ conjugirten Durchmessern $S$ zu jeder Richtuny $R$ statt?" 
20 2. Steiner, algeb. Curven, welche Mittelp. haben, u. Eigens. allgem. algeb. Curven.

„Wird die Richtung $\boldsymbol{R}$ der Tangenten auf alle mögliche Weise geändert, oder lä/st man den $\boldsymbol{P o l}_{\infty} \boldsymbol{P}_{\infty}$ die Gerade $\boldsymbol{G}_{\infty}$ durchlaufen, so haben die zugehörigen ersten Polurén den Mittelpunct $\mathfrak{M}$ gemein und bilden zudem einen Curvenbüschel $\boldsymbol{B}\left(\boldsymbol{C}^{m-1}\right)$ mit $(m-1)^{2}$ Grundpuncten $p$ und $\left.p_{1},{ }^{*}\right)$ welche paurweise Gegenpuncte oder Endpuncte von $\frac{1}{2}(m-1)^{2}$ Sehnen $p_{1}$ sind (vergl. \$. 3.). Die Curven dieses Büschels haben im Ganzen $3(m-2)^{2}$ Doppelpuncte $\mathfrak{p}_{2}$, welche paarweise einzelnen Curven $C^{m-1}$ angehören und Gegenpuncte sind; nur wenn ein $\mathfrak{p}_{2}$ in $\mathfrak{M}$ oder in $G_{\infty}$ liegt, kann er vereinzelt dastehen. In $G_{\infty}$ liegen $2(m-2)$ Doppelpuncte $\mathfrak{p}_{2}$, duher ist die Zahl jener Paare (oder die Zahl der Curven $C^{m-1}$, welche $2 \mathfrak{p}_{2}$ haben $)=\frac{1}{2}(m-2)(3 m-8)$." Werden hierbei die zwei Zahlformen von $m$ berücksichtigt, $m=2 \mu$ und $m=2 \nu-1$, so hat man statt $\operatorname{des} \boldsymbol{B}\left(\boldsymbol{C}^{m-1}\right)$ folgende zwei :

$$
\text { a) } \left.\boldsymbol{B}\left(\boldsymbol{C}^{2 \mu-1}\right) \text { und } \boldsymbol{\beta}\right) \boldsymbol{B}\left(\boldsymbol{C}^{2 v-2}\right) \text {. }
$$

Bei $(\alpha)$ gehört der Mittelpunct $\mathfrak{M}$ mit zu den $(m-1)^{2}$ Grundpuncten (weil jede $C^{2 \mu-1}$ durch ihren eigenen Mittelpunct geht); bei $(\beta)$ dagegen gehört $\mathfrak{M}$ zu den $3(m-2)^{2}=3(2 v-3)^{2}$ Doppelpuncten $\mathfrak{p}_{2}$, weil nothwendig eine der Curven, etwa $C_{0}^{2 v-2}$, durch $\mathfrak{M}$ gehen und ihn daher sum $\mathfrak{p}_{2}$ haben mu/s (\$. 1.). Diese besondere Cürve $C_{0}^{2 \nu-2}$ entspricht derjenigen Richtung $R$, welche durch die Wendetangente der Basis $C^{m}=C^{2 v-1}$ im Puncle $\mathfrak{M}$ gegeben ist. In diesem Falle ist die Ansahl der Paare Doppelpuncte $=2(\nu-2)(3 \nu-4)+\frac{1}{2}$, wo der Bruch, $\frac{1}{2}$, den in $\mathfrak{M}$ liegenden. $\mathfrak{P}_{2}$ anzeigt.

$\mathrm{Zu}$ den zuletzt angegebenen Eigenschaften gesellen sich in besondern Fällen noch andere Umstände, wie an folgenden einfachsten Beispielen zu sehen ist.

1. Ist die gegebene Curve $C^{m}$ nur eine $C^{3}$, so gehen nach jeder Richtung $\boldsymbol{R}$ je 6 Tangenten, deren 6 Berührungspuncte in einem mit $C^{3}$ concentrischen Kegelschnitt $C^{2}$ liegen und zugleich die Endpuncte dreier Durchmesser des letztern sind. Für alle Richtungen $\boldsymbol{R}$ entsteht ein $\boldsymbol{B}\left(\boldsymbol{C}^{2}\right)$, die

*) Diese $(m-1)^{2}$ Puncle sind als die erste Polarenveloppe der Geraden $G_{\infty}$ in Bezug auf die gegebene Curve $C^{\prime n}$ anzusehen (s. obigen Monatsbericht). Die übrigen Polarenveloppen, die $2^{\text {te }}, 3^{\text {te }}, \ldots,(m-1)^{\text {ste }}$ haben alle den Punct $\mathfrak{M}$ zum Mittelpunct, und erscheinen überhaupt in specieller Form; so z. B. reducirt sich die letzte, oder $(m-1)^{\text {ste }}$ Polarenveloppe, die im allgemeinen Falle eine Curve von der $(m-1)^{\text {sten }}$ Classe und vom $2(m-2)^{\text {ten }}$ Grade ist, hierbei auf den blofsen Mittelpunct $\mathfrak{M}$, indem nach obiger Angabe, die letzte Polare, $\boldsymbol{C}^{1}$, stets durch $\mathfrak{M}$ geht. 
alle mit $C^{3}$ concentrisch sind, und deren 4 Grundpuncte aus zwei Paar Gegenpuncten, etwa $p$ und $p_{1}, r$ und $r_{1}$, bestehen und somit die Ecken eines Parallelogramms sind. Die Curven $\boldsymbol{B}\left(\boldsymbol{C}^{2}\right)$ haben im Ganzen nur 3 Doppelpuncte $\mathfrak{p}_{2}$, aber keine von ihnen kann hier $2 \mathfrak{p}_{2}$ baben, sondern die $3 \mathfrak{p}_{2}$ gehören drei verschiedenen speciellen $C^{2}$ an, wovon die eine, $C_{1 \text {, }}^{2}$, aus den Diagonalen, $p p_{1}$ und $r r_{1}$, und jede der zwei andern, $C_{a}^{2}$ und $C_{b}^{2}$, aus einem Paar Gegenseiten des Parallelogramms besteht, so dafs jene ihren $\mathfrak{p}_{2}$ in $\mathfrak{M}$ und jede von diesen ihren $\mathfrak{p}_{2}$ in $\boldsymbol{G}_{\infty}$ zu liegen hat. Die $\boldsymbol{C}_{0}^{2}$ entspricht der Richtung der Wendetangente der Curve $C^{3}$ im Puncte $\mathfrak{M}$; und von $\boldsymbol{C}_{a}^{2}$ und $\boldsymbol{C}_{b}^{2}$ entspricht jede der Richtung der zwei Gegenseiten, aus welchen die andere besteht, so dafs zwischen ihnen Reciprocität statt findet.

2. Ist die gegebene Curve eine $C^{4}$, so gehen nach jeder Richtung $\boldsymbol{R}$ je 12 Tangenten, deren 12 Berührungspuncte in einer mit $C^{4}$ concentrischen Curve $\boldsymbol{C}^{3}$ liegen. Die allen Richtungen $\boldsymbol{R}$ entsprechenden $\boldsymbol{C}^{3}$ bilden ein $\boldsymbol{B}\left(\boldsymbol{C}^{\mathbf{3}}\right)$ mit 9 Grundpuncten, wovon einer $\mathfrak{M}$ selbst ist, die 8 übrigen dagegen 4 Paar Gegenpuncte $p$ und $p_{1}$, oder die Endpuncte von 4 Sebnen $p p_{1}$ sind. Die Curven $\boldsymbol{B}\left(\boldsymbol{C}^{3}\right)$ haben im Ganzen 12 Doppelpuncte $\mathfrak{p}_{2}$; nämlich es giebt unter ihnen 4 solche, $C_{2}^{3}$, wovon jede zwei $\mathfrak{p}_{2}$, und 4 solche, $C_{1}^{3}$, wovon jede nur ein $\mathfrak{p}_{2}$ hat. Jede der $4 C_{2}^{3}$ zerfällt in $C^{2}+C^{1}$, nämlich $C^{1}$ ist je eine der 4 Sehnen $p p_{1}$ und $C^{2}$ geht durch die Endpuncte der je 3 übrigen Sehnen. Die einzelnen Doppelpuncte der $4 C_{1}^{3}$ liegen im Unendlichen, in $\boldsymbol{G}_{\infty}$.

\section{\$. 10.}

Aus dem Bisherigen ist zu sehen, dafs eine höhere Curve $C^{m}$, welche einen Mittelpunct $\mathfrak{M}$ hat, offenbar in ihrem ganzen Wesen der Art beschränkt wird, dafs sie durch keine projectivische Umwandlung aus einer allgemeinen Curve gleichen Grades, etwa $C_{1}^{m}$, entstanden sein, noch in eine solche übergehen kann. Denn wird $\boldsymbol{C}^{m}$ von irgend einem Puncte $\boldsymbol{P}$ des Raumes aus, auf eine beliebige Ebene projicirt, so behält die neue Curve $C_{1}^{m}$ immerhin die folgende, sie modificirende besondere Eigenschaft, nämlich (\$.9.):

, ,Da/s es in ihrer Ebene einen solchen Punct $\mathfrak{M}_{1}$ giebt, durch welchen $\frac{1}{2} m(m-2)$ ihrer Doppeltangenten $\mathfrak{I}_{2}$ gehen, deren $m(m-1)$ Berührungspuncte, $b$ und $b_{1}$ von jeder $\mathfrak{I}_{2}$, in einer neuen Curve $C_{1}^{m-2}$ liegen; und da/s die Berührungspuncte der noch übrigen, aus $\mathfrak{M}_{1}$ an $C_{1}^{m}$ gehenden, $m$ einfachen Tangenten in einer Geraden $G$ liegen, welche jede $\mathfrak{I}_{2}$ in demjenigeń Puncte g schneidet, der mit $\mathfrak{M}_{1}$ zu ihren beiden 
Berührungspuncten $b$ und $b_{1}$ harmonisch ist, also $g, b, \mathfrak{M}_{1}, b_{1}$ vier harmonische Puncte sind; da/s ferner jede durch $\mathfrak{M}_{1}$ gezogene Tansversale $S_{1}$ die Curve $C_{1}^{m}$ in $\frac{1}{2} m$ solchen Punctenparen $q$ und $q_{1}$ schneidet, wovon jedes Paar zu $\mathfrak{M}_{1}$ und dem Puncte $g_{1}$, in welchem $\mathcal{S}_{1}$ jene Gerade $G$ schneidet, harmonisch sind, also je 4 Puncte $q, \mathfrak{M}_{1}, q_{1}, g_{1}$ harmonisch sind, und da/s die beiden Tangenten in jedem Punctenpaar $q$ und $q_{1}$ sich auf $G$ schneiden; und da/s weiter, wenn man umgekehrt aus irgend einem Puncte $P$ in der Geraden $G$ die $m(m-1)$ Tangenten an die Curve $C_{1}^{m}$ legt, dann deren Berührungspuncte paarweise, $q$ und $q_{1}$, mit $\mathfrak{M}_{1}$ in Geraden $S_{1}$ liegen, wovon jede von $G$ im $4^{\text {ten }}$ harmonischen Punct $g_{1}$ geschnitten wird, also $q, \mathfrak{M}_{1}, q_{1}$ und $g_{1}$ harmonisch sind, und da/s endlich die durch alle $m(m-1)$ Berührungspuncte gehende Curve $C_{1}^{m-1}$, d. $i$. die erste Polare des Pols $P$ in Bezug auf die Basis $C_{1}^{m}$, den Punct $\mathfrak{M}_{1}$ und die Gerade $G$ gleicherweise zum harmonischen Pol und zur harmonischen Geraden hat, wie die Basis selbst, und da/s es sich mit der $2^{\text {ten }}, \mathbf{3}^{\text {ten }}, \ldots$ Polaren auch eben so verhält."

Auch in Rücksicht der übrigen obigen Sätze geht das eigentlich Wesentliche der Mittelpuncts-Eigenschaften, bei gleicher perspectivischer Umwandlung nicht verloren, sondern es stellt sich nur in der neuen Figur in scheinbar allgemeinerer Form dar. So z. B. geht der Satz in (\$. 3.) verbunden mit (\$.9.), durch solche Umwandlung, in folgenden über:

,Zieht man durch einen Punct $\mathfrak{M}_{1}$
a) $\mu(\mu+2)-1, \quad$ oder
B) $\nu(\nu+1)-2$

unbegrenzte Gerade $S_{1}$ nach boliebigen Richtungen, schneidet dieselben durch eine andere willkürliche Gerade $G$ in Puncten $g$ und bestimmt sodann in jeder Geraden $S_{1}$ irgend ein Paar solche Puncte $p$ und $p_{1}$, die zu $g$ und $\mathfrak{M}_{1}$ zugeordnete harmonische Puncte sind, so gehen durch alle Puncte $p$ und $p_{1}$ ein Curvenbüschel $B\left(C^{2 \mu}\right)$ oder $B\left(C^{2 \nu-1}\right)$, welche nebstdem noch

$$
\text { a) } \left.2(\mu-1)^{2}, \quad \text { oder } \beta\right) 2(\nu-1)(\nu-2)+1
$$

andere Puncte $q$ und $q_{1}$ gemein haben, die gleichfalls paarweise in neuen durch $\mathfrak{M}_{1}$ gehenden Geraden $S_{1}$ liegen, welche von derselben Geraden $G$ im $4^{\text {ten }}$ harmonischen Punct $g_{1}$ geschnitten werden, so da/s $q, \mathfrak{M}_{1}, \varphi_{1}, g_{1}$ harmonisch sind. Dabei hat jede Curve des Büschels den Punct $\mathfrak{M}_{1}$ und die Gerade $G$, in gleichem Sinne wie vorhïn, zum harmonischen Pol und zur harmonischen Geraden. Im Falle $(\beta)$ gehen alle Curven $C^{2 \nu-1}$ durch 
2. St einer, algeb. Curven, welche Mittelp. haben, u. Eigens. allgem. algeb. Curven. 23

den Punct $\mathfrak{M}_{1}$ und von jeder liegt ein Wendepunct in ihm. In beiden Fälen haben die Curven (als $\boldsymbol{B}\left(C^{m}\right)$ aufgefa/st) im Ganzen $3(m-1)^{2}$ Doppelpuncte $\mathfrak{p}_{2}$, wovon $2(m-1)$ auf die Gerade $G$ fallen und im Allgemeinen einzeln eben so vielen Curven angehören, wogegen die übrigen, zu $\frac{1}{2}(m-1)(3 m-5)$ Paaren, je derselben Curve angehören und jedes Paar in einer neuen, durch $\mathfrak{M}_{1}$ gehenden Geraden $S_{1}$ liegt, welche gleicherweise von der Geraden $G$ im $4^{\text {ten }}$ harmonischen Punct geschnitten wird. Im Falle $(\alpha)$ hat eine der Curven $C^{2 \mu}$ den Punct $\mathfrak{R}_{1}$ zum Doppelpunct $p_{2}$."

Aus der tief eingreifenden Wirkung des Mittelpuncts im vorstehenden ersten Satze erkennt man, dafs, aufser der Curve zweiten Grads $C^{2}$, nur noch die Curve dritten Grads $C^{3}$ durch ihn keine ihr freies Wesen störende Modification erleidet, da sie keine eigentliche Doppeltangenten hat. Und in der That kann auch jede gegebene Curve $C_{1}^{3}$ durch Projection in eine solche andere Curve $\boldsymbol{C}^{3}$ umgewandelt werden, welche einen Mittelpunct $\mathfrak{M}$ hat, und zwar im Allgemeinen auf mehrfache Art, wie aus Folgendem erhellen wird.

\section{\$. 11.}

Ist $\mathfrak{M}_{1}$ ein Wendepunct, $=\mathfrak{w}$, einer beliebigen Curve $C_{1}^{3}$, so zerfällt seine erste Polare bekanntlich in die zugehörige Wendetangente $\mathfrak{B}$ und in eine bestimmte andere Gerade $\boldsymbol{H}$; nämlich von den 6 Tangenten, welche von einem beliebigen Puncte aus an die Curve gehen, fallen hier 3 auf $\mathfrak{Z}$ und ihre 3 Berührungspuncte liegen also auch in $\mathfrak{W}$, und daher mũssen die Berührungspuncte der 3 übrigen Tangenten ebenfalls in einer Geraden $\boldsymbol{H}$ liegen, welche mit $\mathfrak{W}$ zusammen die erste Polare des Puncts $\mathfrak{w}$ in Bezug auf $\boldsymbol{C}_{1}^{3}$ vorstellt. Diese Gerade $\boldsymbol{H}$ hat ferner die Eigenschaft: „da/s sie jede durch w gezogene Transversale $\boldsymbol{S}$ in demjenigen Puncte h schneidet, der zu den 3 Puncten $p, \mathfrak{w}, p_{1}$, in welchem $\mathbf{S}$ von der Curve $C_{1}^{3}$ geschnitten wird, der vierte (stets dem w zugeordnete) harmonische Punct ist." Demgemäfs soll die Gerade $\boldsymbol{H}$ die „Harmonische" des Wendepuncts $\mathfrak{w}$ (dessen halbe Polare sie ist) genannt werden.

Diese Eigenschaft enthält das eigentliche Wesen des Mittelpuncts. Denn wird die Curve $C_{1}^{3}$ auf eine andere Ebene so projicirt, dafs die Harmonische $\boldsymbol{H}$ ins Unendliche geht, d. h. dafs ihr in der neuen Ebene die unendlich entfernte Gerade $G_{\infty}$ entspricht, so ist die Projection des Puncts $\mathfrak{w}\left(\mathfrak{M}_{1}\right)$ der Mittelpunct $\mathfrak{M}$ der neuen Curve $\boldsymbol{C}^{3}$. 
24 2. Steiner, algeb. Curven, welche Mittelp. haben, u. Eigens. allgem. algeb. Curven.

Demnach kann die Curve $C_{1}^{3}$ auf mehrfache Art so projicirt werden, dafs die neue Curve $C^{3}$ einen Mittelpunct $\mathfrak{M}$ erhält, nämlich jeder $\mathfrak{w}$ von jener kann in $\mathfrak{M}$ von dieser übergehen. Und somit ist eine Curve $C^{3}$, welche einen Mittelpunct $\mathfrak{M}$ hat, nur eine solche, bei welcher die Harmonische $\boldsymbol{H}$ eines ihrer Wendepuncte im Unendlichen liegt, $=\boldsymbol{G}_{\infty}$ ist.

Hiernach finden bei der beliebigen Curve $C_{1}^{3}$, in Rücksicht jedes Wendepuncts $\mathfrak{w}$ und der zugehörigen Harmonischen $\boldsymbol{H}$, analoge Sätze statt, wie oben (\$. 9. III. 1.) und (\$. 10.), z. B.

,Zieht man durch einen Wendepunct wo der beliebigen Curve $C_{1}^{3}$ irgend eine Transversale $\mathbf{S}$, so schneidet sie die Curve in zwei solchen Puncten $q$ und $q_{1}$, deren zugehörige Tangenten einander in irgend einem Puncte $\boldsymbol{P}$ auf der Harmonischen $\boldsymbol{H}$ von $\mathfrak{w}$ treffen; auch schneiden die beiden Tangenten die Curve in zwei neuen Puncten $r$ und $r_{1}$, welche mit $\mathfrak{w}$ in einer neuen Geraden $\boldsymbol{S}_{1}$ liegen." Und umgekehrt: ,Werden bei einer beliebigen Curve $C_{1}^{3}$ aus irgend einem Puncte $P$ in der Harmonischen $\boldsymbol{H}$ eines ihrer Wendepuncte $\mathfrak{w}$ die 6 Tangenten an die Curve gezogen, so liegen deren 6 Berührungspuncte paarweise, $q$ und $q_{1}$, in drei durch $\mathfrak{w}$ gehenden Geraden $q q_{1}$, und die durch alle 6 Berührungspuncte gehende Polare $C^{2}$ hat den Punct $\mathfrak{w}$ und die Gerade $\boldsymbol{H}$ zu Pol und Polare; und ferner: die 6 Tangenten schneiden die Curve in neuen 6 Puncten, welche eben so paarweise $\left(r\right.$ und $\left.r_{1}\right)$ in drei durch w gehenden Geraden $\boldsymbol{r r}_{1}$ und zudem alle 6 in einer Curve $C_{1}^{2}$ liegen, die gleichfalls $\mathfrak{w}$ und $\boldsymbol{H}$ su Pol und Polare hat, und die sich mit jener Polare $C^{2}$ in zwei Puncten berührt. Ist $\boldsymbol{P}$ insbesondere der gemeinschaftliche Schnittpunct von 3 solchen Harmonischen $H$, deren zugehörige $3 \mathfrak{w}$ in einer Geraden liegen, so müssen die Berührungspuncte der aus $\boldsymbol{P}$ an die Curve yelegten 6 Tangenten auch dreimal paurweise in drei Geraden qq, liegen, welche beziehlich durch die $3 \mathfrak{w}$ gehen; eben so die 6 Puncte $r$ und $r_{1}$, in welchen die 6 Tangenten die Curve schneiden." U. s. w.

Von den 9 Wendepuncten $\mathfrak{w}$ einer beliebigen Curve $C_{1}^{\grave{3}}$ sind im Allgemeinen 3 reell und 6 imaginär; eben so verhält es sich mit den zugehörigen 9 Harmonischen $\boldsymbol{H}$, sowie auch mit den 9 Wendetangenten $\mathfrak{W}$. Es ist von Interesse, das gegenseitige Verhalten dieser Elemente in folgenden besondern Fällen näher zu betrachten.

Wenn die Curve $C_{1}^{3}$ einen Doppelpunct $\mathfrak{p}_{2}$ hat, so kann er unter drei verschiedenen Formen erscheinen, nämlich erstens als Schnitt zweier reeller 
Zweige, so dafs ihm zwei reelle Tangenten, etwa $\mathfrak{D}$ und $\mathfrak{S}$, zugehören; zweitens als Rückkehrpunct $\mathfrak{r}$, der aus dem vorigen dadurch entsteht, dafs die Schleife der Curve sich bis auf den Punct $f_{2}$ zusammenzieht, wobei dann die Tangenten $\mathfrak{D}$, und $\mathfrak{S}$ in die Rückkehrtangente $\Re$ zusammenfallen; drittens als sogenannter isolirter oder conjugirter Punct $\pi_{2}$, durch den kein reeller Zweig mehr geht und dem daher auch keine reelle eigentliche Tangenten zugehören. Demgemäfs ist nun auch das Verhalten der vorgenannten Elemente verschieden.

I. Hat die Curve $\boldsymbol{C}_{1}^{3}$ einen $\mathfrak{p}_{2}$ mit zwei zugehörigen reellen Tangenten $\mathfrak{D}$, und $\mathfrak{S}$, so fallen von den 9 Wendepuncten 6 in $\mathfrak{p}_{2}$, wovon 4 imaginär und 2 , die $\mathfrak{q}$ und $\mathfrak{s}$ heifsen mögen, reell sind. Von diesen zwei reellen Wendepuncten $\mathfrak{q}$ und $\mathfrak{B}$, in $\mathfrak{p}_{2}$, sind jene Tangenten $\mathfrak{D}$. und $\mathfrak{S}$ als die zugehörigen Wendetangenten, so wie verwechselt zugleich als die zugehörigen Harmonischen $(\boldsymbol{H})$ anzusehen, so dafs also die Wendetangenten und Harmonischen zu diesen zwei Puncten verwechselt anfeinanderfallen. Von den noch übrigen 3 Wendepuncten, die nicht in $\mathfrak{p}_{2}$ liegen, sind zwei imaginär, $i \mathfrak{w}$, und einer reell, $\mathfrak{w}$. Die aus $\mathfrak{p}_{2}$ durch diesen reellen $\mathfrak{w}$ gezogene Gerade heifse $\boldsymbol{W}$. Von den aus $\mathfrak{w}$ an die Curve gehenden drei Tangenten, durch deren Berührungspuncte die Harmonische $\boldsymbol{H}$ bestimmt wird, fallen hier zwei auf die Gerade $\boldsymbol{W}$, und ihre zwei Berührungspuncte müssen als in $\mathfrak{p}_{2}$ liegend gedacht werden, die dritte, eigentliche Tangente heifse $\mathfrak{H}$.und ihr Berührungspunct $b$, so ist also die Gerade $\mathfrak{p}_{2} \boldsymbol{b}$ die Harmonische $\boldsymbol{H}$ von $\mathfrak{w}$, und folglich gehen alle drei reellen Harmonischen, $\mathfrak{D}, \mathfrak{S}$ und $\boldsymbol{H}$, durch $\mathfrak{p}_{2}$ (eben so auch die imaginären).

Welche Modification hierbei die vorstehenden Sätze erleiden, ist leicht zu sehen. Ein neu hinzutretender Umstand ist der: ,Dafs die Geraden $\boldsymbol{W}$ und $\boldsymbol{H}$ zu den Tangenten $\mathfrak{D}$ und $\mathfrak{S}$ harmonisch sind, d.h.da/s $\mathfrak{D}, \boldsymbol{W}$, S, H vier harmonische Strahlen sind." Auch findet dabei ein umfassenderer Satz statt, der sich aus andern Betrachtungen ergiebt, nämlich:

,Zieht man aus dem Doppelpunct $\mathfrak{p}_{2}$ irgend zwei zu $\mathfrak{D}$ und $\mathfrak{S}$ zugeordnete harmonische Strahlen $W_{1}$ und $H_{1}$, welche die Curve $C_{1}^{3}$ in swei neuen Puncten, etwa $\mathfrak{w}_{1}$ und $b_{1}$, schneiden werden, so ist der Ort der diese Puncte verbindenden Geraden $\mathfrak{w}_{1} b_{1}$ eine Curve $C^{2}$, welche insbesondere sowohl die Tangenten $\mathfrak{D}$ und $\mathfrak{S}$ als auch die vorgenannte Tangente $\mathfrak{H}(\mathrm{oder} \mathfrak{w} b)$ berührt."

Crelle's Journal f. d. M. Bd. XLVII. Heft 1. 
26 2. St einer, algeb. Curven, welche Mittelp. haben, u. Eigens. allgem. algeb. Curven.

II. Hat die Curve $C_{1}^{3}$ einen Rückkehrpunct $r$, so sind 8 Wendepuncte als in ihm liegend zu denken (zu den 6 vorigen gesellen sich noch die genannten zwei $i \mathfrak{w}$ ); von denselben sind 6 imaginär und 2 reell, und zwar haben die letztern, da' sie von den vorigen Puncten $\mathfrak{q}$ und $\mathfrak{g}$ herkommen, die Rückkehrtangente $\Re$ sowohl zur gemeinsamen Harmonischen als zur gemeinsamen Wendetangente (weil $\mathfrak{D}$ und $\mathfrak{S}$ sich in $\Re$ vereinigt haben), so dafs sie also durch diese ihnen zugehörigen Elemente nicht mehr zu unterscheiden sind, nur etwa noch dadurch, dafs man sie als den verschiedenen Zweigen der Curve angebörend auffafst; in manchem Betracht sind sie daher nur als ein Punct zu achten. Der $9^{\text {te }}$ und eigentliche Wendepunct ist der vorige reelle, $\mathfrak{w}$, aber die vorhin aus ihm an die Curve gehende Tangente $\mathfrak{S}(=\mathfrak{w} b)$, fallt hier auch noch auf die Gerade $W(=\mathfrak{r w})$, so dafs jetzt alle 3 Tangenten, durch deren Berührungpuncte die Harmonische $\boldsymbol{H}$ von $\mathfrak{w}$ geht; in $\boldsymbol{W}$ und ihre drei Berührungspuncte in $\mathfrak{r}$ vereinigt sind, allein wenn nun auch hiedurch die $\boldsymbol{H}$ nicht mehr bestimmt wird, so folgt doch andererseits aus ihrer harmonischen Lage, dafs sie mit $\mathfrak{L}$ und $\mathfrak{S}$ zugleich in die Rückkehrtangente $\mathfrak{R}$ übergehen mufs. Demnach gehen in diesem Falle die drei reellen Harmonischen nicht allein alle durch den Rückkehrpunct $\mathfrak{r}$, sondern sie fallen alle drei in die Rückkehrtangente $\Re$ zusammen.

Aus den obigen Sätzen ergeben sich hier folgende specielle Sätze.

„Jede durch den Wendepunct wo gezogene Gerade $\mathbf{S}$ wird von der Curve $C_{1}^{3}$ und deren Rückkehrtangente $\Re$ in 4 harmonischen Puncten geschnitten; d. h. wird $S$ von $C_{1}^{3}$ in den Puncten $q, \mathfrak{w}, q_{1}$ und von $R$ im Puncte $r$ geschnitten, so sind immer $q, \mathfrak{w}, q_{1}, r$ vier harmonische Puncte." „Die in den beiden Puncten $q$ und $q_{1}$ an die Curve gelegten Tangenten treffen sich allemal in irgend einem Puncte $P$ auf der Rückkehrtangente $\Re$; und umgekehrt: werden aus irgend einem Puncte $P$ der Rückkehrtangente $\Re$ die zwei, nicht auґ $\Re$ fallenden, Tangenten an die Curve gelegl, so liegen ihre Berührungspuncte $q$ und $q_{1}$, stets in einer durch den Wendepunct $\mathfrak{w}$ gehenden Geraden S." Und ferner: „Zieht man durch den Rükkehrpunct $\mathfrak{r}$ irgend swei zu $\Re$ und $W$ zugeordnete harmonische Strahlen $Q$ und $Q_{1}$, so schneiden diese die Curve in zuei neuen Puncten $q$ und $q_{1}$, welche jedesmal mit dem Wendepunct $\mathfrak{w}$ in einer Geraden $\mathbf{S}$ liegen." - „Wenn ferner die durch $\mathfrak{w}$ gezogene Transversale $\boldsymbol{S}$ insbesondere der Rückkehrtangente $\Re$ parallel ist, so stehen die Schnitte $q$ und $q_{1}$ gleichweit von $\mathfrak{w}$ ab; und wenn $\mathcal{S}$ mit einer der drei 
Asymptoten der Curve parallel ist, so liegt einer der beiden Puncte und $q_{1}$, er heifse für einen Augenblick $q_{0}$, so liegt $q_{0}$ in der Mitte zwischen $\mathfrak{w}$ und $r$; wnd daher auch umgekehrt: zieht man durch die Mitte der Geraden $W(=\mathfrak{n w})$ eine Gerade $Q_{0}$ parallel $R$, so schneidet sie die Curve in 3 Puncten $q_{10}$ und die aus $w$ durch dieselben gezogenen 3 Geraden wo $q_{0}$ sind den drei Asymptoten parallel, und die in den Puncten $q_{0}$ an die Curve gelegten Tangenten treffen sich mit den respectiven Asymptoten auf der Rückkehrtangente $\Re . "$

III. Hat die Curve $C_{1}^{3}$ einen isolirten Punct $\pi_{2}$, so sind in demselben 6 imaginäre Wendepuncte zu denken, die übrigen drei Wendepuncte w sind reell und liegen in einer Geraden. Von den aus jedem dieser drei reellen $w$ an die Curve zu legenden 3 Tangenten fallen, wie oben (I.), zwei auf die Gerade $\mathfrak{w} \pi_{2}=W$, so dafs ihre beiden Berührungspuncte in $\pi_{2}$ liegen; die dritte Tangente heifse, wie dort, $\mathfrak{H}$ und ihr Berührungspunct $b$, so ist also die Gerade $\pi_{2} b$ die Harmonische $\boldsymbol{H} \mathrm{zu} \mathfrak{w}$, und folglich gehen auch hier die Harmonischen $\boldsymbol{H}$ der 3 reellen Wendepuncte $w$ alle drei durch den Doppelpunct $\pi_{2}$. Auch findet hierbei ein analoger Umstand statt, wie bei (I.), nämlich: „Die drei Paar Gerade $W$ und $\boldsymbol{H}$ (aus dem Doppelpunct $\pi_{2}$ durch die Wendepuncle $w$ und durch die Beruhrungspuncte $b$ der aus w gelegten Tangenten $H$, gezogen) sind 3 Paar conjugirte Strahlen eines elliptischen Strahlsysterns, oder bilden elliptische Involution. Construirt man irgend ein anderes Paar conjugirte Strahlen desselben Strahlsysterns, etwa $W_{1}$ und $H_{1}$, so schneiden sie die Curve in swei neuen Puncten $\mathfrak{w}_{1}$ und $b_{1}$, und der Ort der sie verbindenden Geraden $\mathfrak{w}_{1} b_{1}$ ist eine Curve $C^{2}$, welche nothwendig auch jene drei Tangenten $\mathfrak{H}$ berührt." „Hier liegt $\cdot \pi_{2}$ innerhalb der Curve $C^{2}$; bei (I.) liegt, $p_{2}$ au/serhalb derselben, und bei (II.) reducirt sich dieselbe auf den Rückkehrpunct $\mathfrak{x} . "$

Übrigens haben die 3 Paar Gerade $\boldsymbol{W}$ und $\boldsymbol{H}$ eine noch innigere Beziehung zu einander; nämlich jede Gerade $\boldsymbol{H}$ ist vierte barmonische zu den 3 Geraden $W$, und umgekehrt jede Gerade $W$ ist vierte harmonische zu den $3 \boldsymbol{H}$. Dies Verhalten kann wie folgt klar gemacht werden. Soll zu drei durch einen Punct gehenden gegebenen Geraden $a, b, c$ eine vierte harmonische Gerade bestimmt .werden, so sind 3 Lösungen möglich, indem sowobl $a b a c$, als $a b c \beta$, als a $a b c$ harmonisch sein können; und werden sodann die drei neuen Geraden $\alpha, \beta, \gamma$ als gegeben angesehen, so sind umgekehrt jene erstern Geraden $a, b, c$ die ihnen entsprecbenden vierten Harmonischen, so 
28 2. St einer, algeb. Curven, welche Mittelp. haben, u. Eigens. allgem. algeb.Curven.

dafs also zugleich auch $\alpha \beta a \gamma, \alpha \beta \gamma b, \alpha c \beta \gamma$ harmonisch sind. Dabei ist, wie man sieht, jedes Paar conjugirte Gerade, wie etwa $a$ und $\alpha$, sowohl zu $b$ und $c$, als auch zu $\beta$ und $\gamma$ harmonisch. Diese nämliche Beziehung haben nun auch die 3 Paar Gerade $W$ und $H$, wenn man die $3 W$ als $a, b$, und die $3 \boldsymbol{H}$ als $\alpha, \beta, \gamma$ ansiebt. Wenn insbesondere zwei Paar conjugirte Gerade unter sich rechtwinklig sind, wenn etwa $(a \alpha)$ und $(b \beta)$ rechte Winkel sind, so ist auch $(c \gamma)$ ein rechter Winkel, und alsdann bilden je zwei nach der Reihe $a \gamma b \alpha c \beta a$ aufeinander folgende Gerade einen Winkel von 30". Dabei ist das genannte Strahlsystem ein rechtwinkliges, so dafs jeder Winkel $\left(W_{1} H_{1}\right)$ ein rechter ist. - Ein Theil des obigen Satzes ist bereits von Möbius in seiner Abhandlung, "über Linien dritter Ordnung" bewiesen worden; ich bin jedoch nicht erst dadurch zu dem Satze gelangt.

Soll nun, in Rücksicht auf die vorstehenden drei besondern Fälle I, II und III, die jedesmalige gegebene Curve $C_{1}^{3}$ durch Projection in eine solche andere Curve $C_{0}^{3}$ umgewandelt werden, welche einen (reellen) Mittelpunct $\mathfrak{M}$ hat, so kann $C_{1}^{3}$ auf folgende Weise projicirt werden.

A. Bei I. auf zwei wesentliche verschiedene Arten, nämlich entweder

a) so, dafs die Harmonische $\boldsymbol{H}$ des reellen Wendepuncts $\mathfrak{w}$ in die Gerade $\boldsymbol{G}_{\infty}$ und dadurch $\mathfrak{w}$ in $\mathfrak{M}$ übergeht, wobei also auch der Doppelpunct $\mathfrak{p}_{2}^{0}$ der neuen Curve in $\boldsymbol{G}_{\infty}$ zu liegen kommt, und daher die Tangenten $\mathfrak{D}$ und $\mathfrak{S}$ in zwei parallele, gleichweit von $\mathfrak{M}$ abstehende Asymptoten $\mathfrak{D}_{0,}$ und $\mathfrak{S}_{11}$ übergehen, so wie $\mathfrak{S}$ in die dritte, durch $\mathfrak{M}$ selbst gehende, Asymptote $\mathfrak{S}_{0}$ übergeht; oder

B) so, dafs die Tangente $\mathfrak{Z}$ (oder $\mathfrak{S}$ ) in $\boldsymbol{G}_{\infty}$ und damit ihr Berührungspunct $\mathfrak{q}$ in $\mathfrak{M}$ übergeht, mithin auch $\mathfrak{M}$ in $\boldsymbol{G}_{\infty}$ liegt und $\mathfrak{M}_{\infty}$ heifsen.mag, wobei $\mathfrak{S}$ in die einzige sichtbare Asymptote $\mathfrak{S}_{0}$ der neuen Curve ũbergeht, indem die beiden andern auf $\boldsymbol{G}_{\infty}$ fallen, und wobei die 3 Geraden $\mathfrak{S}_{(0}, \boldsymbol{W}_{0}, \boldsymbol{H}_{0}$ parallel werden, $\mathfrak{\Im}_{0}$ in der Mitte zwischen den beiden andern liegt, und daher durch die Mitte der Tangente $\mathfrak{S}_{0}=\mathfrak{w}_{0} b_{0}$ geht.

B. Bei II. kann nur so projicirt werden, dafs die Rückkehrtangente $\Re$ in $\boldsymbol{G}_{\infty}$ übergeht, aber dadurch gehen der Wendepunct $\mathfrak{w}$ und der Rückkehrpunct $\mathfrak{r}$, wofern man die im letztern vereinten zwei reellen Wendepuncte $\mathfrak{q}$ und $\mathfrak{z}$ nur für einen achtet, zumal in Mittelpuncte der neuen Curve $C_{0}^{3}$ über, so dafs diese also zwei Mittelpuncte hat, wovon der eine, $\mathfrak{M}$, ihr eigentlicher Wendepunct $\mathfrak{w}_{0}$, der andere, $\mathfrak{M}_{\infty}$, ihr im Unendlichen liegender Rückkehr- 
punct $\mathfrak{r}_{0}$ ist. Hier hat die Curve $C_{0}^{3}$ keine eigentliche Asymptote, sondern alle drei Asymptoten fallen auf $\boldsymbol{G}_{\infty}$.

C. Bei III. kann dreifach, aber auf gleichbedeutende Art, projicirt werden, nämlich so, dafs je eine der drei Harmonischen $\boldsymbol{H}$ in $\boldsymbol{G}_{\infty}$ und der ihr zugehörige Wendepunct $\mathfrak{w}$ in $\mathfrak{M}$ übergeht; dabei kommt also der Doppelpunct $\pi_{2}^{0}$ der neuen Curve $\boldsymbol{C}_{0}^{3}$ jedesmal in $\boldsymbol{G}_{\infty}$ zu liegen und die dem jedesmaligen $\mathfrak{w}$ zugehörige Tangente $\mathfrak{S}$ geht in die einzige reelle und eigentliche Asymptote $\mathfrak{S}_{0}$ der Curve $\boldsymbol{C}_{0}^{3}$ über.

Aus diesem Verhalten der besondern Elemente, sind, zu nachheriger Benutzung, noch folgende zwei Sätze hervorzuheben:

$1^{\circ}$. „Soll eine Curve $C^{3}$ einen Mittelpunct und sugleich auch einen (aber nur einen) Doppelpunct haben, so mu/s der letstere nothwendig in Unendlichen, auf $G_{\infty}$, liegen, dabei kunn er aber, je nach Umstünden, entweder $\mathfrak{p}_{2}$, oder $\mathfrak{x}$, oder $\pi_{2}$ sein."

$2^{\circ}$. „Hat eine Curve $C^{3}$ einen im Unendlichen liegenden Mittelpunct, $\mathfrak{M}_{\infty}$, so ist derselbe nothwendig sugleich ein Doppelpunct und zwar ein Doppelpunct erster Art, $\mathfrak{p}_{2}$ (I.), oder insbesondere ein Rückkehrpunct $\mathfrak{r}$ (II.), und so ist die Gerade $G_{\infty}$ nothwendig Tangente in demselben (also $\mathfrak{2}$ oder $\mathfrak{S}$, oder insbesondere $\mathfrak{R}$ )."

\$. 12.

Die eben betrachteten Eigenschaften besonderer Curven dritten Grads gewähren eine Ergänzung der Sätze in \$. 7., so wie weitere Folgerungen aus denselben.

Da in Rücksicht derjenigen Schaar Curven dritten Grads, $\boldsymbol{S}\left(\boldsymbol{C}^{3}\right)$, welche durch gegebene 6 Puncte $\boldsymbol{p}$ gehen und Mittelpuncte $\mathfrak{M}$ haben, der Ort dieser Mittelpuncte eine Curve fünften Grads $\mathfrak{M}^{5}$ ist (\$. 7. I.), die im Allgemeinen fünf Púncte im Unendlichen, auf $\boldsymbol{G}_{\infty}$, hat, so folgt: dafs sich unter $\operatorname{der} \boldsymbol{S}\left(\boldsymbol{C}^{3}\right)$ fünf solche $C_{0}^{3}$ befinden, welche jene unendlich entfernten Puncte beziehlich zu Mittelpuncten $\left(\mathfrak{M}_{\infty}\right.$ ) und somit zugleich zu Doppelpuncten $\mathfrak{p}_{2}$ (oder insbesondere $\mathfrak{r}$ ) haben, und in denselben von der Geraden $\boldsymbol{G}_{\infty}$ berührt werden (\$. 11. $2^{\circ}$.). Aufser diesen $5 C_{0}^{3}$ giebt es unter der $\boldsymbol{S}\left(\boldsymbol{C}^{\mathbf{3}}\right)$ keine, welche nur einen Doppelpunct hat, wohl aber befinden sich unter denselben 36 solche, wovon jede zwei Doppelpuncte hat, indem sie aus $C^{2}+C^{1}$ besteht, was bereits oben (\$. 7. I.) nachgewiesen worden. Also:

„Unter der Schaar Curven $C^{3}$, welche durch gegebene 6 Puncte $p$ gehen und Mitlelpuncte $\mathfrak{M}$ haben, giebt es im Allgemeinen fünf solche, 
30 2. St einer, algeb.Curven, welche Mittelp. haben, u. Eigens. allgem. algeb. Curven.

$C_{0}^{3}$, deren Mittelpuncte $\left(\mathfrak{M}_{\infty}\right)$ im Unendlichen liegen, daher zugleich Doppelpuncte $\mathfrak{p}_{2}$ (oder $\mathfrak{x}$ ) sind und die Gerade $G_{\infty}$ sur sugehörigen Tangente haben. Die $\boldsymbol{S}\left(\boldsymbol{C}^{3}\right)$ haben im Ganzen 77 Doppelpuncte; jedoch giebt es blo/s die genannten $5 C_{0}^{3}$, wovon jede uur einen Doppelpunct hat, dagegen 36 solche, wovon jede in $C^{2}+C^{1}$ serfällt und daher zusei Doppelpuncte hut."

Durch Projection folgt:

„Soll eine beliebige Curve $C^{3}$ durch gegebene 6 Puncte p gehen und eine yegebene Gerade $\boldsymbol{H}$ zur Harmonischen eines ihrer Wendepuncte $\mathfrak{w}$ haben, so ist der Ort dieses $\mathfrak{w}$ eine Curve fünften Grads, $\mathfrak{M}^{5}$, welche durch die 6p, so wie durch 51 andere leicht construirbare Puncte geht (\$. 7. I.). Unter dieser Schair Curven $C^{3}$ giebt es 36 solche, wovon jede aus $C^{2}+C^{1}$ besteht und somit zwei Doppelpuncte hat; hingegen giebt es nur 5 solche $C_{0}^{3}$, wovon -jede blo/s einen Doppelpunct $\mathfrak{p}_{2}$ (oder $\mathfrak{x}$ ) hat, und zwar liegen diese 5 Doppelpuncle in der Geraden $H$, sind ihre Schnitte mit der Ortscurve $\mathfrak{M}^{5}$, und in jedem ist $\boldsymbol{H}$ Tangente an die zugehörige Curve $C_{0}^{3}$." Oder man kann auch sagen: „Sind 6 Puncte $p$ und eine Gerade $\boldsymbol{H}$ gegeben, so giebt es fünf solche Curven $C_{0}^{3}$, welche durch die $6 p$ gehen und die $H$ sur Tangente in einem Doppelpuncte $\mathfrak{p}_{2}$ haben." Hieraus und aus dem Umstande: „Dafs die Curve $C_{0}^{3}$ bestimmt ist, wenn sie durch gegebene 6 Puncte $p$ gehen und einen gegebenen siebenten Punct q zum Doppelpunct, oder wenn sie durch gegebene 5 Puncte $p$ gehen, einen gegebenen sechsten Punct q sum Doppelpunct und in diesem eine gegebene Gerade $\mathfrak{D}$ zur Tangente haben soll," können weiter folgende Sätze geschlossen werden.

I. „Soll eine Curve $C_{0}^{3}$ durch gegebene 6 Puncte $p$ gehen und einen Doppelpunct $\mathfrak{p}_{2}$ haben, dessen eine Tangente $\mathfrak{D}$ durch einen gegebenen siebenten Punct $q$ geht, so ist der Ort des Doppelpuncts $\mathfrak{p}_{2}$ eine Curve $\boldsymbol{g}^{\text {ten }}$ Grads, $G^{7}$, welche sowohl den Punct $q$ als die 6 Puncte $p$ zu Doppelpuncten hal und wobei die eine Tangente jedes Doppelpuncts $p$ auf die Gerade pq fïllt; - und ferner ist der Ort der andern Tangente $\mathfrak{S}$ des Doppelpuncts $\mathfrak{p}_{2}$ der Curve $C_{0}^{3}$ eine Curve 25 $^{\text {ster }}$ Classe, $\boldsymbol{K}^{25}$." Von der Curve $\boldsymbol{G}^{7}$ sind viele andere specielle Puncte leicht zu construiren. Ferner: „Unter der Schaar Curven $C_{0}^{3}$ giebt es, im Allgemeinen, 18 solche, welche stat! des Doppelpuncts $\mathfrak{p}_{2}$ einen Rükkehrpunct $\mathfrak{x}$ haben, dessen (Rückkehr-) Tangente $\Re$ also ebenfalls durch den gegebenen 
2. Steiner, algeb.Curvén, welche Mittelp. haben, u. Eigens. allgem. algeb. Curven. 31

Punct q geht und die Curve $K^{25}$ berührt (indem $\mathfrak{D}$ und $\mathfrak{S}$ in $\Re$ vereinigt sind \$. 11.) und zudem auch die Curve $G^{7}$ im Puncte $\mathfrak{r}$ berührt." Und ferner:

„Soll eine Curve $C_{0}^{3}$ durch gegebene 6 Puncte $p$ gehen und einen Doppelpunct $\mathfrak{p}_{2}$ haben, dessen Tangenten $\mathfrak{D}$ und $\mathfrak{S}$ beziehlich durch zwei andere gegebene Puncle y und s gehen, so finden, im Allgemeinen, 25 Lösungen stutt."

II. „Soll eine Curve $C_{0}^{3}$ durch gegebene $F$ Puncle $p$ gehen und einen Doppelpunct $\mathfrak{p}_{2}$ haben, so ist der Ort dieses Doppelpuncts eine Curve $6^{\text {ten }}$ Gruds, $G^{6}$, welche die 7 Puncte $p$ zu Doppelpuncten hat, und so ist der gemeinsame Ort seiner beiden Tangenten $\mathfrak{Z}$ und $\mathfrak{S}$ eine Curve

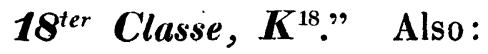

„Soll eine Curve $C_{0}^{3}$ durch gegebene $\gamma$ Puncte $p$ gehen und einen Doppelpunct $\mathfrak{p}_{2}$ haben, dessen eine Tangente $\mathfrak{D}$ durch einen achten gegebenen Punct q geht, so finden, im Allgemeinen, 18 Lösungen statt."

III. „Soll eine Curve $C_{10}^{3}$ durch gegebene 6 Puncte $p$ gehen und einen Rückkehrpunct $\mathfrak{r}$ haben, so ist der Ort des letzlern eine Curve $\boldsymbol{6}^{\text {ten }}$ Grads, welche jene 6 Puncte $p$ zu Doppelpuncten hat; und so ist der Ort der Rückkehrtangente $\Re$ eine Curve $18^{\text {ter }}$ Classe." Daher:

„Soll eine Curve $C_{0}^{3}$ durch gegebene 6 Puncte $p$ gehen und einen Rückkehrpunct $\mathfrak{x}$ haben, dessen Tangente $\Re$ durch einen gegebenen siebenten Punct $r$ geht, so giebt es, im Allgemeinen, 18 Lösungen."

Hieran schliefse ich noch folgende Aufgabe.

IV. Wenn beliebige 6 Puncte $p$ gegeben sind, so ist jeder andere Punct $q$ der Ebene Doppelpunct einer durch jene 6 Puncte gehenden bestimmten Curve $C_{0}^{3}$. Werden nun die Doppelpuncte nach den zwei Arten durch $\mathfrak{p}_{2}$ und $\mathcal{H}_{2}$ unterschieden ( $\$$. 11.), so kann man fragen: ,in welchen Theilen oder Regionen der Ebene der Punct y liegen müsse, damit er ein $\mathfrak{p}_{2}$ oder ein $\pi_{2}$ sei?" Bestehen die Grenzen dieser Regionen nur allein aus der vorgenannten Curve $6^{\text {ten }}$ Grads (III.)? 
32 2. St einer, algeb. Curven, welche Mittelp. Laben, u. Eigens. allgem. algeb. Curven.

\section{Vorkommen der Curven, welche Mittelpuncte haben, bei Betrachtung allgemeiner Curven.}

Innere Polaren.

\$. 13.

Durch jeden Punct $\boldsymbol{P}$ in der Ebene eines Kegelschnitts $\boldsymbol{C}^{2}$ läfst sich immer eine, aber im Allgemeinen nur eine solche (reelle oder imaginäre) Sehne $\boldsymbol{a} a_{1}$ ziehen, welche durch den Punct $\boldsymbol{P}$ gehälftet wird. Sobald zwei solche Sehnen durch denselben Punct $\boldsymbol{P}$ möglich sind, so finden zugleich unendlich viele statt, und dann ist $\boldsymbol{P}$ der Mittelpunct von $\boldsymbol{C}^{2}$.

Diese Betrachtung kann auch auf die höheren Curven ausgedehnt werden. Zieht man durch einen beliebigen. Punct $\boldsymbol{P}$ in der Ebene einer gegebenen Curve $\boldsymbol{C}^{m}$ irgend eine Gerade $\boldsymbol{S}$, so schneidet sie die Curve in $m$ Puncten; nun kann man verlangen, die Gerade soll so gezogen werden, dafs von den $m$ Schnittpuncten irgend zwei, etwa $a$ und $a_{1}$, gleichweit von $\boldsymbol{P}$ abstehen, und zwar auf entgegengesetzten Seiten von $\boldsymbol{P}$ liegen (nicht in einem Berührungspuncte vereinigt sind). Kürze halber soll jede Gerade $\boldsymbol{S}$, welche ein solches Paar Schnittpuncte enthält, schlechthin eine „Sehne" und die Puncte $a$ und $a_{1}$ sollen die Endpuncte der Sehne heifsen; und wenn eine Gerade zugleich zwei Paar solche Schnittpuncte enthält, etwa $a$ und $a_{1}, b$ und $b_{1}$, so soll sie „Doppelseline" genannt und durch $\boldsymbol{S}_{2}$ bezeichnet werden; solche $\boldsymbol{S}_{2}$ hat also auch zwei Paar Endpuncte. Gleicherweise können insbesondere auch dreifache, vierfache, etc. Sehnen vorkommen.

Über die Anzahl aller Sehnen $\boldsymbol{S}$, welche durch denselben Pol $\boldsymbol{P}$ gehen und über die Lage ihrer Endpuncte $a$ und $a_{1}$ hat man den folgenden Satz:

I. „Durch jeden Punct $P$ in der Ebene einer gegebenen Curve $\boldsymbol{C}^{m}$ gehen im Allgemeinen $\frac{1}{2} m(m-1)$ Sehnen $S$, und ihre $m(m-1)$ Endpuncte ( $a$ und $a_{1}$ ) liegen allemal in einer um einen Grad niedrigern Curve $\boldsymbol{J}^{m-1}$, welche nothwendigerweise den Pol $\boldsymbol{P}$ zum Mittelpunct hat." *)

*) Der Beweis dieses Satzes ergiebt sich unter andern durch folgende genmetrische Anschauung. Man denke sich die Curve $\boldsymbol{C}^{m}$ in ihrer Ebene, um den festen Pol $\boldsymbol{P}$, um $180^{\circ}$ herumbewegt und bezeichne sie in der neuen Lage durch $C_{1}^{m}-$ oder, was auf dasselbe hinauskommt, man denke sich zu $\boldsymbol{C}^{m}$ die ihr, in Bezug auf den Punct $\boldsymbol{P}$, symmetrisch gleiche $C_{1}^{m}$, so dafs $C^{m}+C_{1}^{m}$ als eine Curve $C^{2 m}$ anzusehen sind, welche $\boldsymbol{P}$ zum Mittelpunct hat, und wobei also jeder Punct $p$ in $C^{m}$ seinen Gegenpunct $p_{1}$ in $C_{1}^{m}$ hat und auch umgekehrt, - so haben die Curven $C^{m}$ und $C_{1}^{m}$ parallele Asymptoten, von 
Die genannten Endpuncte machen gerade die volle Zahl Schnittpuncte beider Curven aus. Findet sich insbesondere, dafs durch einen Pol $\boldsymbol{P}$ mehr als $\frac{1}{2} m(m-1)$ Sehnen $\boldsymbol{S}$ gehen, ja sobald nur eine Sehne mehr durchgebt, so gehen alsdann unendlich viele hindurch, so dafs die gegebene Curve $C^{m}$ selbst den Punct $\boldsymbol{P}$ zum Mitelpunct hat.

Beachtet man von allen durch den Punct $\boldsymbol{P}$ gehenden Geraden nur diejenigen, $\boldsymbol{T}$, bei welchen von den $m$ Schnitten mit der Curve $\boldsymbol{C}^{m}$ ebenfalls zwei gleichweit von $\boldsymbol{P}$ abstehen, aber auf derselben Seite von $\boldsymbol{P}$ liegen sollen, also zu einem Berührungspunct der Geraden $\boldsymbol{T}$ vereinigt sind: so finden bekanntlich $\boldsymbol{m}(\boldsymbol{m}-1)$ solche Tangenten $\boldsymbol{T}$ statt, deren $m(\boldsymbol{m}-1)$ Berührungspuncte in einer Curve $\boldsymbol{A}^{m-1}$ liegen, welche die erste Polare des Pols $\boldsymbol{P}$ in Bezug auf die gegebene Curve $C^{m}$ heifst (s. den voranstehenden Monatsbericht).

Wegen Übereinstimmung dieser letzten Bedingung mit der vorigen, soll fortan die obige Curve $\boldsymbol{J}^{m-1}$ (wenn auch in anderer Hinsicht nicht ganz passend) die ,innere Polare," hingegen die Curve $A^{m-1}$ die „äu/sere" oder schlechthin nur die erste Polare des Pols $\boldsymbol{P}$ in Bezug auf die Basis $\boldsymbol{C}^{m}$ genannt werden. Beide Polaren sind also immer vom gleichen Grad. Aufserdem haben sie, unter andern, folgende wesentliche Beziehung zu einander.

II. „Die beiden Polaren $A^{m-1}$ und $J^{m-1}$ jedes Puncts $P$ in Bezuy auf dieselbe gegebene Curve $C^{m}$ haben m-1 gegenseitige Schnittpuncte inn Unendlichen, auf der Gerıden $G_{\infty}$, daher müssen ihre Asyınptoten parweise parallel und die zu jeden Paar gehörigen müssen gleichzeitig reell oder imaginär sein; und daher müssen ferner die noch übrigen $(m-1)(m-2)$ Sclınitte beider Polaren in einer Curve vom $(m-2)^{t e n}$ Grad, $C^{m-2}$ liegen."

\section{\$. 14.}

In Rücksicht der innern Polare sind zunächst verschiedene besondere Umstände zu erörtern, welche zum Theil zu interessanten Resultaten führen. Nämlich man kann fragen: welchen Einflufs es auf die Polare $J^{m-1}$ habe, oder wie sie sich gegen die Basis $\boldsymbol{C}^{\prime n}$ verhalte, wenn der Pol $\boldsymbol{P}$ in der

ihren $m^{2}$ gegenseitigen Schnittpuncten liegen somit $m$ in der Geraden $G_{s}$, daher müssen die übrigen $m(m-1)$ Schnitte in einer Curve $(m-1)^{\text {ten }}$ Grads $J^{m-1}$ liegen, und zwar müssen sie paarweise Gegenpuncte in Bezug auf $\boldsymbol{P}$ sein (denn ist a ein Schnilt von $\boldsymbol{C}^{\prime n}$ und $C_{1}^{m}$, so mufs auch sein Gegenpunct $a_{1}$ in beiden Curven zugleich liegen), somit müssen diese Schnitte die Endpuncte von $\frac{1}{2} m(m-1)$ Sehnen aa der Curve $C^{m}$ (auch $\operatorname{der} \boldsymbol{C}_{1}^{m}$ ) sein, und folglich mufs die Curve $\boldsymbol{J}^{m-1}$ den Pol $\boldsymbol{P}$ zum Mittelpunct haben.

Crelle's Journal f. d. M. Bd. XLVII. Heft 1. 
34 2. St einer, algeb. Curven, welche Mittelp. haben, u. Eigens. allgem. algeb. Curven.

letztern selbst liegt, oder insbesondere ein singulärer Punct derselben ist? oder ferner: welche Bewandtnifs es mit der Polare $J^{m-1}$ habe, wenn die Basis von specieller Form ist, etwa in Theile zerfällt? oder ferner: ob es in der Ebene der Basis solche besondere Pole gebe, für welche die innere. Polare eine eigenthümliche Form erhält, in Theile zerfällt? u. s. w. Die wesentlichsten Fälle der Art sollen im Folgenden kurz angedeutet werden.

I. Verhalten der innern Polare, wenn der Pol $P$ in der Basis $C^{m}$ selbst liegt.

Hierbei sind wieder die beiden Zahlformen $m=2 \mu$ und $m=2 \nu-1$ zu unterscheiden, so dafs man es mit den zwei inneren Polaren

$$
J^{2 \mu-1} \text { und } \mathbf{J}^{2 v-2}
$$

zu thun hat, wovon die erstere immer durch ihren eigenen Mittelpunct oder Pol $\boldsymbol{P}$ geht und auch sonst noch sich verschieden von der andern verhält.

1. Wenn der Pol $\boldsymbol{P}$ ein beliebiger Punct der Basis $C^{m}$ ist:

$\alpha)$ so hat $\boldsymbol{J}^{2 \mu-1}$ die Tangente von $\boldsymbol{C}^{m}$ im Puncte $\boldsymbol{P}$ zur Wendetangente, wt (\$. 9.).

2. Wenn $\boldsymbol{P}$ insbesondere ein Wendepunct der Basis $\boldsymbol{C}^{m}$ ist: $\alpha)$ so hat $\mathbf{J}^{2 \mu-1}$ die zugehörige Wendetangente mit der Basis gemein.

3. Wenn $\boldsymbol{P}$ insbesondere ein a) so hat $\boldsymbol{J}^{2 \mu-1}$ in $\boldsymbol{P}$ einen dreifachen $w p$, mit drei $w t$, von welchen sie fünfpunctig berührt wird (\$.1.).

\section{Wenn $\boldsymbol{P}$ insbesondere ein}

a) so hat $\boldsymbol{J}^{2 \mu-1}$ in $\boldsymbol{P}$ einen dreifachen Wendepunct mit drei Wendetangenten, so dafs sie von jeder der letzlern daselbst fünfpunctig berührt wird, wie vorhin (3).

$\beta)$ so hat $\boldsymbol{J}^{2 v-2}$ den $\boldsymbol{P}$ zum $d p$ mit zwei wt, wovon die eine auf die $w t$ der Basis fällt.

\section{Doppelpunct der Basis ist:}

(3) so hat $\boldsymbol{J}^{2 v-2}$ den $\boldsymbol{P}$ wiederum zum $d p$ mit zwei $w t$, welche auf die beiden Tangenten der Basis fallen.

\section{Ruckkehrpunct der Basis ist:}

$\beta)$ so hat $\boldsymbol{J}^{2 v-2}$ in $\boldsymbol{P}$ die $r t$ der Basis zur doppelten $r t$ und doppelten. $w t$, nämlich sie berührt daselbst die rt der Basis doppelt, mit zwei $Z$ weigen, und somit auch sich selbst.

Ist die gegebene Basis z. B. nur vom vierten Grad, $C^{4}$, so besteht die innere Polare $J^{3}$ in den beiden Fällen (3. $\left.\alpha\right)$ und $(4 . \alpha)$ aus drei Geraden, 
2. St einer, algeb.Curven, welche Mittelp. haben, u. Eigens. allgem. algeb. Curven. 35

3J' $\boldsymbol{J}^{1}$, die durch $\boldsymbol{P}$ gehen, nämlich aus drei Doppelsehnen $a u_{1}$ oder $\boldsymbol{S}_{2}$, bei denen das eine Paar Endpuncte, $b$ und $b_{1}$, in $\boldsymbol{P}$ vereinigt sind. Und dabei hat die äufsere Polare $\boldsymbol{A}^{3}$ mit der Basis beziehlich den Doppel-oder Rückkehrpunct nebst den zugehörigen Tangenten ( $\mathcal{L}$, und $\mathfrak{S}$, bei 3., oder $\Re$ bei 4.) gemein. Vermöge des obigen Satzes (\$.13. II.) folgt: „Da/s die drei Geraden, $3 J^{1}$, aus denen die innere Polare besteht, oder die durch $\boldsymbol{P}$ gehenden drei Doppelsehnen $\boldsymbol{S}_{2}=a a_{1}$, beziehlich den drei Asymptoten, $3 \boldsymbol{A}_{s}$, der äu/sern Polare $A^{3}$ parallel sein müssen; und da/s umgekehrt, wenn man durch $\boldsymbol{P}$ mit einer Asymptote von $\boldsymbol{A}^{3}$ eine Gerade $\boldsymbol{S}$ parallel zieht, diese von der Basis $C^{4}$ in zwei von $\boldsymbol{P}$ gleichweit abstehenden Puncten a und $a_{1}$ geschnitten wird."

5. Wenn endlich $\boldsymbol{P}$ insbesondere ein $(\boldsymbol{m}-1)$ facher Punct der Basis $\boldsymbol{C}^{m}$ ist:

,so besteht sowohl die innere als äu/sere Polare, $J^{m-1}$ sowohl als $A^{m-1}$, aus den $m-1$ Tangenten der Basis im Puncte P."

II. Verhalten der innern Polare, wenn die Basis aus Theilen besteht.

Die Basis $\boldsymbol{C}^{m}$ kann auf mannichfache Weise in Theile zerfallen, d. h. aus zwei oder mehren Curven niedrigerer Grade, oder selbst nur aus Geraden bestehen, wobei dann der obige Satz (\$.13.) immerhin bestehen bleibt; was unter andern zu folgenden speciellen Sätzen führt.

\section{Wenn die Basis $C^{m}$ aus $m$ Geraden $G$ besteht.}

„Sind in einer Ebene beliebige $m$ Gerade $G$ gegeben und man zieht durch irgend einen Pol $\boldsymbol{P}$ zwischen je zwei Geraden diejenige Sehne $\boldsymbol{S}$ oder aa $a_{1}$, welche von $\boldsymbol{P}$ gehälftet wird, was im Ganzen $\frac{1}{2} m(m-1)$ Sehnen au giebt, so liegen ihre $m(m-1)$ Endpuncte, a und $a_{1}$, allemal in einer Curve $(m-1)^{s t e n}$ Grads, $J^{m-1}$, welche $\boldsymbol{P}$ zum Mittelpunct hat."

2. Wenn die Basis $C^{m}$ aus zuei Curven $C^{\alpha}$ und $C^{\beta}$ besteht, wo $\alpha+\beta=m$.

,Sind in einer Ebene irgend zwei Curven $C^{\alpha}$ und $C^{\beta}$ gegeben und man zieht durch einen beliebigen Pol $\boldsymbol{P}$ die $\frac{1}{2} \alpha(\alpha-1)$ Sehnen aa in der Curve $C^{\alpha}$, so wie die $\frac{1}{2} \beta(\beta-1)$ Selınen bb $b_{1}$ in der Curve $C^{\beta}$, und ferner die $\alpha . \beta$ Sehnen ab zuischen beiden Curven, (d.h. solche Gerade $a b$, die den einen Endpunct $a$ in $C^{\alpha}$, den andern $b$ in $C^{\beta}$ und ihre 
36 2. Steiner, algeb.Curven, welche Mittelp. haben, u. Eigens. allgem. algeb. Curven.

Mitte in $P$ haben), so liegen die Endpuncle aller dieser Selinen, was susammen $\alpha(\alpha-1)+\beta(\beta-1)+2 \alpha \beta=m(m-1)$ Endpuncte sind, allemal in einer Curve $(\alpha+\beta-1)^{\text {sten }}$ oder $(m-1)^{\text {sten }}$ Grads, $J^{m-1}$, welche den Pol $\boldsymbol{P}$ zum Mittelpunct hat."

Darin ist der besondere Satz enthalten: „Du/s durch jeder Punct $\boldsymbol{P}$ in der Ebene zweier beliebigen Curven $C^{\alpha}$ und $C^{\beta}$, in Allgeineinen, $\alpha . \beta$ solche Sehnen ab möglich sind, welche den einen Endpunct in der einen, den andern in der andern Curve und ihre Mitle in jenem Puncle P haben."

Ferner ist daraus leicht der folgende Satz herzuleiten:

,Sind in einer Ebene irgend zwei Curven $C^{\alpha}$ und $C^{\beta}$ gegeben, die einander in $\alpha . \beta$ Puncten $c$ schneiden, und zieht man durch einen beliebigen Pol $P$ die $\frac{1}{2} \alpha(\alpha-1)$ Sehnen a $a_{1}$ in der Curve $C^{\alpha}$, so wie die $\frac{1}{2} \beta(\beta-1)$ Sehnen $b b_{1}$ in' der Curve $C^{\beta}$, so liegen die Endpuncte beider Systeme Sehnen mit jenen Schnittpuncten $c$, was zusammen $\alpha(\alpha-1)+\beta(\beta-1)+\alpha \beta=m(m-1)-\alpha \beta$ Puncte uusmacht, allemal in einer Curve $(\alpha+\beta-1)^{\text {sten }}$ Grads, $J^{m-1}$, welche den Pol $\boldsymbol{P}$ sum Mittelpunct hat."

III. Lage oder Ort des Pols $\boldsymbol{P}$, wenn die innere Polare in Theile zerfallen soll.

Dieser Fall führt schon auf complicirte Untersuchungen, wenn die gegebene Basis nur von niedrigem Grade, nur vom dritten oder vierten Grad ist, wie aus nachstehenden Betrachtungen erhellen wird.

\section{§. 15.}

I. Ist die gegebene Basis nur vom dritten Grad, $C^{3}$, also die innere Polare jedes Pols $\boldsymbol{P}$ ein Kegelschnitt $\boldsymbol{J}^{2}$, so kann dieser möglicherweise nur in zwei Gerade zerfallen, und es ist die Frage, ob dieses Zerfallen wirklich statt, finden könne, und wo dabei der Pol $\boldsymbol{P}$ liegen müsse, oder welchen Ort er habe? In der That stellt sich heraus, dafs dieses Zerfallen auf zwei verschiedene Arten geschehen kann, und demgemäfs auch zwei verschiedene Örter vorhanden sind, und zwar wie folgt.

„Der Ort des Pols, dessen innere Polare $J^{2}$ in zwei Gerade zerfällt, besteht aus zwei getrennten Curven, nümlich:

A. aus der gegebenen Basis $C^{3}$ selbst, und

B. aus einer bestimnten Curve zweiten Grads, $\boldsymbol{E}^{2}$, welche die zweite Polare der Geraden $G_{\infty}$ in Bezug auf die Basis $C^{3}$ ist (s. obig. Monuts- 
bericht), oder welche die Enveloppe aller Durchmesser von $C^{3}$ ist." Nämlich schneidet man die Curve $C^{3}$ mit einer beliebigen Transversale $\mathfrak{D}$ und bestimmt zu den drei Schnitten den Schwerpunct $d$ : so liegen alle Schwerpuncte $\boldsymbol{d}$ von je einem System paralleler Transversalen $\mathfrak{D}$ in einer Geraden $\boldsymbol{D}$, welche Durchmesser der Curve $C^{3}$ heifst, und wobei die Richtung der Transversalen, die ,conjugirte Richtung" des Durchmessers genannt werden soll. Alle Durchmesser $\boldsymbol{D}$ der Curve $\boldsymbol{C}^{3}$, wozu insbesondere auch ihre Asymptoten $\boldsymbol{A}_{s}$ gehören, berühren nun den genannten Kegelschnitt $\boldsymbol{E}^{2}$. Sind die $\mathbf{3 \boldsymbol { A } _ { s }}$ alle reell, so ist $\boldsymbol{E}^{2}$ diejenige dem Asymptotendreieck eingeschriebene Ellipse, welche dessen Seiten in ihren Mitten berührt; und schneiden sich insbesondere die $3 \boldsymbol{A}_{s}$ in einem Puncte, so reducirt sich $\boldsymbol{E}^{2}$ auf diesen Punct, so dafs alle Durchmesser durch denselben gehen. Ist dagegen nur eine $\boldsymbol{A}_{s}$ reell, so ist $\boldsymbol{E}^{2}$, im Allgemeinen, eine Hyperbel, welche diese $\boldsymbol{A}_{s}$ in einem leicht zu construirenden Puncte berührt.

Über die beiden Örter $(\boldsymbol{A}$.) und (B.) sind die nähern Umstände folgende.

II. 1. Liegt der Pol $\boldsymbol{P}$ in der Basis $\boldsymbol{C}^{3}$ selbst ( $\boldsymbol{A}$.), so fällt von den ihm zugehörigen drei Sehnen $S$, oder $a a_{1}, b b_{1}, c c_{1}$ die eine, etwa $c c_{1}$, auf die ihm zugehörige Tangente, wobei ihre Endpuncte $c$ und $c_{1}$ sich mit $P$ vereinigen, daher als in den beiden andern Sehnen liegend anzusehen sind, etwa $c$ in $a a_{1}$ und $c_{1}$.in $b b_{1}$, so dafs also der Kegelschnitt $J^{2}$ in die beiden Sehnen $a c a_{1}$ und $b c_{1} b_{1}$ übergeht, die wir zur Unterscheidung durch $S_{1}$ oder auch nach Umständen durch $\boldsymbol{S}$ und $\boldsymbol{S}_{1}$ bezeichnen wollen. Demnach entsprechen jedem in der Basis liegenden $\boldsymbol{P o l} \boldsymbol{P}$ nur zwei eigentliche Sehnen $\mathbb{S}_{1}$, die dritte fällt auf die Tangente, wird unendlich klein, reducirt sich auf ihren Berührungspunct $\boldsymbol{P}$. Denkt man sich nun zu demselben P.ol zugleich auch die äufsere Polare $A^{2}$, so folgt (\$. 13. II.):

,Für jeden in der Basis $C^{3}$ liegenden Pol sind die ihm entsprechenden zwei Sehnen $\boldsymbol{S}$ und $\boldsymbol{S}_{1}$ den Asymptoten seiner äu/sern Polare $A^{2}$ parallel.”, ,Daher sind beide Sehnen reell oder imaginär, je nachdem die Polare $A^{2}$ Hyperbel oder Ellipse ist, und auch umgekehrt; und wenn insbesondere $\boldsymbol{A}^{2}$ Parabel ist, so fallen die Sehnen $\mathbf{S}$ und $\boldsymbol{S}_{1}$ aufeinander, und auch umgekehrt." Es giebt im Ganzen nur 6 solche besondere Pole $\boldsymbol{P}$, die $\boldsymbol{P}_{0}$ heifsen sollen, für welche die Sehnen $\mathbf{S}$ und $\boldsymbol{S}_{1}$ in eine, ab oder $\boldsymbol{S}_{0}$, zusammenfallen, und womit zugleich die Polare $A^{2}$ Parabel wird, und zuar sind die 6 Pole $\boldsymbol{P}_{0}$ die gegenseitigen Schnitte 
38 g. St einer, algeb. Curven, welche Mittelp. haben, u. Eigens. allgem. algeb. Curven.

der Curven $C^{3}$ und $\left.E^{2}{ }^{*}\right)$ Jede der 6 Sehnen $\boldsymbol{S}_{0}$ hat die Eigenschaft, da/s die in illren Endpuncten $a, b$ an die Basis $C^{3}$ gelegten Tangenten $\boldsymbol{A}, \boldsymbol{B}$ parallel sind. Liegt der Pol $\boldsymbol{P}$ insbesondere in einem Wendepunct $\mathfrak{w}$ der Basis, so fält eine der beiden Sehnen $\mathbf{S}$ und $\boldsymbol{S}_{1}$, etwa $\mathbf{S}$, auf die Wendetangente $\mathfrak{W}$, und alsdann besteht auch $A^{2}$ aus zwei Geraden, nümlich aus $\mathfrak{W}$ und.der Harmonischen $H$ von $\mathfrak{w}$ (\$. 11.), und es ist $\boldsymbol{S}_{1} \# \boldsymbol{H}, \boldsymbol{d} . h$. , in diesem Halle besteht jede der beiden Polaren $\boldsymbol{J}^{2}$ und $A^{2}$ aus zwei Geraden, wovon swei auf $\mathfrak{W}$ fallen und die beiden andern, $S_{1}$ und $H$, parallel sind.

Die Sehnenpaare $\boldsymbol{S}$ und $\boldsymbol{S}_{1}$ sind insgesammt dem folgenden Gesetz unterworfen.

,Alle Sehnen $\boldsymbol{S}_{1}$, in welche die innere Polare, $\boldsymbol{J}^{2}$ zerfällt, wenn der $\boldsymbol{P}$ ol $\boldsymbol{P}$ in der Basis $\boldsymbol{C}^{3}$ selbst liegt, oder was auf dasselbe hinauskommt, alle solche Sehnen aca, deren Mitten, $c$, in der Basis selbst liegen, berühren eine bestimmte Curve $6^{\text {ter }}$ Classe, $\mathcal{S}_{1}^{6}$, und $18^{\text {ten }}$ Grads, G ${ }^{18} . "$

Über das Verhalten dieser Curve gegen die Basis und über andere Eigenschaften derselben, mag hier noch Folgendes hinzugefügt werden.

2. Die Curve $\boldsymbol{S}_{1}^{6}$ berührt die Basis $\boldsymbol{C}^{3}$ in ihren 9 Wendepuncten $\mathfrak{w}$, so wie in ihren 3 unendlich entfernten Puncten $a_{s}$, so da/s sie also die 3 Asymptoten $\boldsymbol{A}_{\text {. }}$ mit ihr yemein hat; aber die $\mathbf{S}_{1}^{6}$ berührt jede dieser $3 \boldsymbol{A}_{s}$ uuch noch in einem bestimmten andern Puncte, so da/s sie dieselben zu Doppeltangenten hat. Da die Basis ebenfalls von der $\boldsymbol{6}^{\text {ten }}$ Classe ist, $\boldsymbol{C}^{3}=K^{6}$, so bestehen die 36 gemeinschaftlichen Tangenten beider Curven blos aus den 9 Wendetangenten $\mathfrak{W}$ und den 3 Asymptoten der $C^{3}$, indem jede dieser 12 Geraden für 3 gemeinschuftliche Tangenten zu zählen ist.

3. Die Curve $S_{1}^{6}$ berührt die oben genannten 6 besondern Sehnen $S_{11}$ in ihren Mitten $\boldsymbol{P}_{0}$ und schneidet somit daselbst die Basis $C^{3}$. Von den

*) In jener Abhandlung, welche der obige Monatsbericht bespricht, wird gezeigt: "Dafs die Curve $E^{2}$ der Ort aller derjenigen Pole $P$ ist, für welche die äufsere Polare $A^{2}$ Parabel wird; und dafs überhaupt die Potare $A^{2}$ Hyperbel, Ellipse oder. Parabel ist, je nachdem der Pol $P$ beziehlieh au/serhalb, innerhalb oder in der Curve $E^{2}$ liegt." Dasselbe gilt also auch für die innere Polare $J^{2}$, da sie stets mit $\boldsymbol{A}^{2}$ ähnlich und ähnlich liegend ist. "Es giebt, im Allgemeinen, uur einen bestimmten Pol $P$, dessen Polaren $A^{2}$ und ${ }^{2}$ Kreise sind." "Liegt der Pol $P$ insbesondere im Mittelpuncte der Curve $E^{2}$, so sind seine Polaren $A^{2}$ und $J^{2}$ der $E^{2}$ ähnlich, mit ihr ähnlich liegend und concentrisch." Das Nähere hierüber sehe man unten, Aufg. and Sätze, welche sich auf die gegenwärtige Abhandlung beziehen. 
2. St einer, algeb. Curven, welche Mittelp. haben, u. Eigens. allgem. algeb. Curven. 39

$3.18=54$ gemeinschaftlichen Puncten beider Curven kennen wir also bereits 30, nümlich die $9 \mathfrak{w}$ und $3 a_{\infty}$, jeden doppelt gezühlt, und die $6 \boldsymbol{P}_{0}$; die 24 übrigen haben die Eigenschaft, da/s sie die einen Endpuncte $a_{1}$ solcher besondern Sehnen aca $a_{1}$ sind, die $\mathfrak{S}_{0}$ heifsen mögen, bei welchen die im andern Endpuncte a und in der Mitte $c$ an die Basis gelegten Tangenten $A$ und $C$ parallel sind, und welche die Curve $S_{1}^{6}$ in den Puncten $a_{1}$ selbst berühren. Durch die 24 Puncte $a_{1}$ können Curven $\mathcal{B}^{\text {ten }}$ Grads gehen.

4. Die 12 gemeinschaftlichen Tangenten der Curven $\boldsymbol{S}_{1}^{6}$ und $\mathbb{E}^{2}$ bestehen: 1) aus den $3 \boldsymbol{A}_{\text {s }}$ der Basis, jede doppelt gezählt, und 2) aus 6 solchen Sehnen $\mathbf{S}_{1}$, welche zugleich Durchmesser der Basis sind; die 6 Mitten $c$ dieser 6 Sehnen liegen in irgend einem Kegelschnitte $C^{2}$.

5. Die Curve $\boldsymbol{S}_{1}^{6}$ hat ferner die Gerade $\boldsymbol{G}_{\infty}$ zur dreifachen Tangente, berührt sie in 3 Puncten $g_{\infty}$. Diese 3 Puncte sind dadurch bestimmt, da/s sie zu den drei Puncten $a_{\infty}$ (2.) die vierten harmonischen Puncte sind; d. h., wenn man durch irgend einen Punct drei Gerade $\boldsymbol{A}, \boldsymbol{B}, \boldsymbol{C}$ den $3 \boldsymbol{A}_{s}$ der $\boldsymbol{C}^{3}$ parallel zieht und $z u$ denselben die 3 vierten harmonischen Strahlen $A_{1}, B_{1}, C_{1}$ bestimmt, so da/s $A B A_{1} C, A B C B_{1}$, $A C_{1} B C$ harmonisch sind, oder auch so, wenn man in dem AsymptotenDreieck $3 A_{s}$ aus den Ecken durch die Mitten der Gegenseiten die 3 Strahlen $A_{1}, B_{1}, C_{1}$ zieht: so sind diese Strahlen nach jenen unendlich entfernten Berührungspuncten $g_{\infty}$ gerichtet. (Die auf diese Weise construirten 3 Strahlen sind dann auch beziehlich den Axen der 3 asymptotischen Parabeln parallel, welche die Curve $\boldsymbol{S}_{1}^{6}$ in den 3 Puncten $g_{\infty}$ fünfpunctig berühren.) Da die Curve $\boldsymbol{S}_{1}^{\mathbf{6}}$ vom $18^{\text {ten }}$ Grad ist, so mufs sie mil der Geraden $\boldsymbol{G}_{\infty}$ aufser den bereits angegebenen 9 Puncten (den $3 g_{\infty}$, doppelt gezählt, und den $3 a_{\infty}$ ), noch 9 andere Puncte, $d_{\infty}$, gemein haben. Diese Puncte $d_{\infty}$ sind dadurch, bestimmt, da/s die zugehörigen Tangenten oder Asymptoten, $\mathfrak{D}_{1}$, der Curve durch diejenigen Puncte, $d_{0}$, der Basis $C^{3}$ gehen, in welchen letztere von einzelnen ihrer Durchmesser, $D_{0}$, berührt wird, und dafs dieselben die diesen Durchmessern conjugirte Richtung haben (I.). Dafs es 9 solche Durchmesser $\boldsymbol{D}_{0}$ giebt, erhellet daraus, dafs sie gemeinschaftliche Tangenten der Curven $C^{3}$ und $E^{2}$ sind, welche 12 gemeinschaftliche Tangenten haben, aber wovon drei die Asymptoten $\boldsymbol{A}_{s}$ der $\boldsymbol{C}^{3}$ sind. Die 9 Asymptoten $\mathfrak{D}_{0}$ sind zugleich solche eigenthümliche Sehnen $\boldsymbol{a d}_{10} a_{1}\left(=S_{1}\right)$, bei welchen die in den End́puncten $a, a_{1}$ und in der Mitte $d_{0}$ an die Basis $C^{3}$ gelegten drei 
40 2. Steiner, algeb. Curven, welche Mittelp. haben, u. Eigens. allgem. algeb. Curven.

Tangenten $\boldsymbol{A}, \boldsymbol{A}_{1}$ und $\boldsymbol{D}_{0}$ sich in irgend einem Puncte $\boldsymbol{Q}$ treffen. Also: „In einer Curve $3^{\text {ten }}$ Grads $C^{3}$ giebt es, im Allgemeinen, 9 solche Trainsversulen $\mathfrak{D}_{1}$, bei welchen von den drei Schnitten der eine, $d_{10}$, in der Mitte zwischen den beiden andern, $a$ ' und $a_{1}$, liegt und wobei die zugehörigen drei Tangenten in irgend einem Puncte $\boldsymbol{Q}$ zusammentreffen, und wo zudem die Tangente $\boldsymbol{D}_{0}$ in mittelsten Schnittpuncte $d_{0}$ zugleich ein Durchmesser der Curve ist." - Die Beziehungen, welche die Curve $\boldsymbol{S}_{1}^{6}$ rücksichtlich der 9 Geraden $\mathfrak{D}_{0}$ und der 9 Puncte $\boldsymbol{Q}$ zu andern, mit der Basis $C^{3}$ innig zusammenhängenden Curven hat, werden hier übergangen und sollen bei einer andern Gelegenheit näher in Betracht kommen.

6. ,Durch jeden beliebigen Punct $\boldsymbol{Q}$ gehen, im Allgemeinen, 6 Sehnen $S_{1}$ oder aca $a_{1}$ und ihre 6 Mitten c liegen allemal in irgend einem Kegelschnitte. Liegt $Q$ in der Basis $C^{3}$ selbst, so wird diese in demselben von dem Kegelschnitte berïhrt." Versetzt man $Q$ ins Unendliche, so fallen von den 6 Sehnen $S_{1}$ drei auf $\boldsymbol{G}_{\infty}$ und die drei übrigen laufen parallel, und ilere Mitten $c$ liegen in dem ihrer Richtung conjugirten Durchmesser $D$, sind dessen Schnitte mit der Basis $C^{3}$. Somit sind von den Tangenten der Curve $\mathcal{S}_{1}^{6}$, oder von den Selonen $\boldsymbol{S}_{1}$, nur je 3 und 3 parallel und ihre Mitten sind jedesmal die 3 Endpuncte des ihre. Richtung conjugirten Durchmessers $\boldsymbol{D}$ der Basis $\boldsymbol{C}^{3}$, und auch umgekehrt.

7. Der Berührungspunct $s$ jeder Sehne $a c a_{1}=S_{1}$ mit ihrer Ortscurve $\boldsymbol{S}_{1}^{6}$ wird durch folgende einfache Construction gefunden. Man lege in ihren Endpuncten $a, a_{1}$ und in ihrer Mitte $c$ an die Basis $C^{3}$ die Tangenten $\boldsymbol{A}, \boldsymbol{A}_{1}$ und $\boldsymbol{C}$; ihre Schnitte $\boldsymbol{A} \boldsymbol{A}_{1}, \boldsymbol{A C}, \boldsymbol{C A _ { 1 }}$ mögen beziehlich $p, q, q_{1}$ heifsen. In $A$ und $A_{1}$ nehme man die Puncte $\mathfrak{p}$ und $\mathfrak{p}_{1}$ so, dafs $q$ und $q_{1}$ die Mitten der Strecken $p \mathfrak{p}$ und $p p_{1}$ sind; ziehe sodann die Geraden $a p_{1}$ und $a_{1} \mathfrak{p}$, nenne ihren Schnitt $r$, so geht die Gerade $p r$ durch den gesuchten Berührungspunct $s$ der Sehne $a u_{1}$. - Hiezu noch die Bemerkung. Die durch die Puncte $p$ und $\mathfrak{p}_{1}$ gezogene Gerade $\boldsymbol{C}_{1}$, - die mit $\boldsymbol{C}$ parallel und mit ihr auf gleicher Seite von $p$ liegt, aber doppelt so weit von $\boldsymbol{p}$ absteht, - schneidet die Sehne $a a_{1}$ in demjenigen Puncte $s_{1}$, welcher mit $s$ zu $a$ und $a_{1}$ harmonisch ist, d. h. $a s a_{1} s_{1}$ sind harmonisch. Geht $C$ insbesondere durch $p$, so fällt also $C_{1}$ auf $C, s_{1}$ in $c$, und $s$ entfernt sich ins Unendliche.

8. Aus (2.) folgt, unter andern, der nachstehende Satz.

,Denkt man sich in derselben Ebene zwei ähnliche Curven dritten Grads, $C^{3}$ und $C_{1}^{3}$, deren homologe Dimensionen sich verhalten, wie $2: 1$, 
2. St ciner, algeb. Curven, welche Mitlelp. haben, u. Eigens. allgem. algeb. Curven. 41

hält die eine, etwa $C^{3}$, in ihrer Laye fest, so kann die andere auf 24 verschiedene Arten so gelegt werden, da/s beide Curven direct (nicht symmetrisch) ähnlich lieyen; einander in irgend einem Paar homologen Puncten $m$ und $m_{1}$ und nebstdem noch in irgend zurei nicht homologen Puncten $n$ und $q_{1}$ berühren." „Durch die 24 Puncte $m$ in der Curve $C^{3}$ können Curven $\mathcal{8}^{\text {ten }}$ Grads gehen; eben so durch die $24 m_{1}$ in $C_{1}^{3}$."

III. Liegt der Pol $\boldsymbol{P}$ in der Curve $\boldsymbol{E}^{2}$ (I. $\boldsymbol{B}$.), so sind die ihm zugehörigen drei Sehnen $S$ oder $a a_{1}, b b_{1}, c c_{1}$ so beschaffen, dafs etwa die drei Endpuncte $\boldsymbol{a}, \boldsymbol{b}, \boldsymbol{c}$ in einer Geraden $\boldsymbol{J}$, und somit auch die drei andern $a_{1}, b_{1}, c_{1}$ in einer Geraden $J_{1}$ liegen, so dafs also unter diesen Umständen die innere Polare $\boldsymbol{J}^{2}$ in die zwei Geraden $\boldsymbol{J}$ und $\boldsymbol{J}_{1}$, zerfällt, welche parallel sind und gleichweit vom Pol $\boldsymbol{P}$ abstehen, und zudem auch projectivisch gleich sind, indem $a b=a_{1} b_{1}, a c=a_{1} c_{1}, b c=b_{1} c_{1}$ ist. In diesem Falle ist die äufsere Polare jedesmal eine Parabel, deren Axe mit den Geraden $J$ und $J_{1}$ parallel ist (II. 1.). Von den in $\boldsymbol{E}^{2}$ liegenden Polen zeichnen sich zunächst folgende durch eigenthümliche Umstände aus. 1) Die schon oben genannten 6 Schnitle $\boldsymbol{P}_{0}$ der Curven $\boldsymbol{C}^{3}$ und $\boldsymbol{E}^{2}$. In jedem derselben wird die Sehne $\boldsymbol{c c}_{\boldsymbol{1}}$ unendlich klein, und daher fallen die Geraden $\boldsymbol{J}$ und $\boldsymbol{J}_{1}$ zugleich mit den Sehnen $a u_{1}$ und $b b_{1}$ (oder oben $S$ und $S_{1}$ ) aufeinander, auf die dortige Sehne $\boldsymbol{S}_{0}$. 2) Ferner giebt es drei solche besondere Pole, die $\boldsymbol{X}, \boldsymbol{Y}, \boldsymbol{Z}$ heifsen mögen, für welche (nicht allein die innere sondern) zugleich auch die äufsere Polare $\boldsymbol{A}^{2}$ (die Parabel) in ein Paar parallele Gerade $\boldsymbol{A}$ und $\boldsymbol{A}_{1}$ zerfällt, welche überdies mit den zugehörigen Geraden $\boldsymbol{J}$ und $\boldsymbol{J}_{1}$ parallel sind. Aufser diesen drei Puncten $\boldsymbol{X}, \boldsymbol{Y}, \boldsymbol{Z}$ giebt es in der ganzen Ebene keinen andern Pol, dessen äufsere Polare $\boldsymbol{A}^{2}$ in zwei parallele Gerade zerfällt.

Über die gesammten Geraden $\boldsymbol{J}, J_{1}$ hat man folgenden Satz.

„Alle Paure Gerade $J$ und $J_{1}$, in welche die innere Polure $J^{2}$ serfällt, uenn der $\boldsymbol{P o l} \boldsymbol{P}$ in der Curve $\boldsymbol{E}^{2}$ liegt, berühren eine Curve $\boldsymbol{6}^{\text {ter }}$ Classe, $\mathbf{J}^{6}$, und $14^{\text {ten }}$ Grads, welche die 6 Sehnen $\mathbf{S}_{0}$ zu Asymptoten und die Gerade $G_{\infty}$ zur vierfachen Tangente hat." Die Curve $J^{6}$ berührt jedoch die Gerade $G_{\infty}$ nicht in vier, sondern in nur, swei verschiedenen Puncten, aber in jedem doppelt, so da/s sie sich in jedem derselben selbst berührt, und zu'cr sind diese zwei Puncle zugleich die gemeinschuftlichen Puncte der Curve $\boldsymbol{E}^{2}$ und der Geraden $\boldsymbol{G}_{\infty}$, oder die unendlich entfernten Puncte der Asymploten von $E^{2}$. ,Wird durch den $P$ ol $P$ mit den zugehöriyen Geraden $J$ und $J_{1}$ eine dritte Gerade, $J_{0}$,

Crelle's Journal f. d. M. Bd. XLVII. Heft 1. 
42 2. Steiner, alyeb. Curven, welche Mittelp. haben, ". Eigens. allgem. algeb. Curven.

parállel gezogen, so ist ihr Ort eine Curve $3^{\text {ter }}$ Classe $J_{0}^{3}$ und $4^{\text {ten }}$ Grads, welche die Gerade $G_{\infty}$ zur Doppeltangente hal, und zwar sie in den eben genaunten zuei Puncten berührt." „Daher ist das ganze System der verschiedenen Paare Gerade $J$ und $J_{1}$ auch so beschaffen, dafs jeder Punct $\mathfrak{P}$ der Ebene, im Allgemeinen, der Mittelpunct eines Kegelschnitts ist, welcher irgend drei der genannten Paare beruht, und surar diejeniyen drei Paure, welche den durch den Punct $\mathfrak{P}$ gehenden drei Geraìen. $J_{0}$ entsprechen."

„Die 36 gemeinschaftlichen Tangenten der Curve.$^{\circ}$ und der Basis $C^{3}$ bestehen aus 18 Paar zusammengehörigen Geraden $J$ und $J_{1} . "$

Wenn die Geraden $J$ und $J_{1}$ Tangenten der Basis $C^{3}$ werden, so vereinigen sich von den obigen drei Puncten $a, b, c$ in $J$ irgend zwei, etwa b und $c$, zu einem Berührungspuncte $(b c)$ oder $\alpha$; eben so die Puncte $b_{1}$ und $c_{1}$ in $J_{1}$ zu einem Berührungspuncte $\left(b_{1} c_{1}\right)$ oder $\alpha_{1}$; und damil fallen die Sehnen $b b_{1}$ und $c c_{1}$ in die Berührungssehne $\alpha \alpha_{1}$ zusammen, die wir durch $\mathfrak{S}_{1}$ und ihre Milte, oder den zugehörigen Pol $\boldsymbol{P}$, durch $\boldsymbol{P}_{1}$ bezeichnen wollen. Die begrenzten gleichen Strecken $a \alpha=a_{1} \alpha_{1}$ von $J$ und $J_{1}$ sollen schlechthin gleiche parallele Tangenten heifsen, aber da ihre Richlungen, von $\alpha$ nach $a$, und von $\alpha_{1}$ nach $a_{1}$, gerade entgegengesetzt sind, so sollen sie ungleichliegend genannt werden. Mit Bezug hierauf, bedingt der letzte Salz den folgenden.

„,Eine beliebige Curve drilten Grads, $C^{3}$, hat, im Allgemeinen, 18 Pair parallele gleiche aber ungleichliegende T'angenten, al und $\alpha_{1} a_{1}$, und die Millen, $P_{1}$, der 18 Berührungssehnen $\mathfrak{S}_{1}\left(=\alpha \alpha_{1}\right)$, liegen in jenem (oben nüher) bestimmten Kegelschnitle $E^{2} . "$

„Von den 36 gemeinschaflichen Tangenten der Curve $J^{(5}$ und der obigen Curve $S_{1}^{6}$ (II.) fallen 12 auf die Gerade $G_{\infty}, 6$ undere sind jene besondern 6 Sehnen $\$_{0}$ (II. 1.), und die noch übrigen 18 bestehen aus 9 Paur zusammengehörigen Geraden $J$ und $J_{1}$." Da die letztern (als Tangenten der $\boldsymbol{S}_{1}^{(i)}$ zugleich 9 Paar parallele und projectivisch gleiche Sehnen $\boldsymbol{S}_{1}$, oder zur Unterscheidung $\boldsymbol{S}_{1}$ und $\boldsymbol{S}_{1}^{1}$, sind, so dafs $J=\boldsymbol{S}_{1}=a b c, J=\boldsymbol{S}_{1}^{1}=a_{1} \boldsymbol{b}_{1} \boldsymbol{c}_{1}$ und $a b=b c=a_{1} b_{1}=b_{1} c_{1}$ ist, wofern $b$ und $b_{1}$ die mitllern Puncte, also die Mitten der Sehnen $\boldsymbol{S}_{1}$ und $\boldsymbol{S}_{1}^{1}$ sind: so hat man weiter, wenn der zugehörige Pol $\boldsymbol{P}$ oder die Milte der Geraden $\boldsymbol{b} \boldsymbol{b}_{1}$ durch $\boldsymbol{P}^{1}$ bezeichnet wird, den folgenden Salz. „In der beliebigen Curve $C^{3}$ giebl es in Ganzen 9 Paar parallele gleiche Sehnen $S_{1}$ und $\mathcal{S}_{1}^{1}$, und die 9 Mitten $P^{1}$ der ihre Mitten $b$ und $b_{1}$ verbindenden Geraden $b_{1}$ liegen in dem oft genunnten Kegelschnille $\boldsymbol{E}^{2}$." 
Rücksichtlich der 42 gemeinschaftlichen Puncte der Curven $J^{9}$ und $C^{3}$ kann bemerkt werden, dafs wenn etwa $a$ ein solcher Punct und $J$ die zugehörige Tangente an $\boldsymbol{J}^{6}$ ist, und man denkt sich das zugehörige Paar Gerade $\boldsymbol{J}$ und $\boldsymbol{J}_{1}$ nebst dessen (in $\boldsymbol{E}^{2}$ liegenden) Pol $\boldsymbol{P}$, dafs alsdann die in den Puncten $P$ und $a_{1}$ an die respectiven Curven $E^{2}$ und $C^{3}$ gelegten Tangenten allemal parallel sind. Daraus schliefst man den folgenden Satz.

„Denkt man sich die gegebene Curve $C^{3}$ in ihrer Ebene un den Mittelpunct, $\boldsymbol{E}$, des Kegelschnitts $\boldsymbol{E}^{2}$ un $180^{0}$ herumbeugegt und beseichnet sie in der neupn Lage durch $C_{1}^{3}$, denkt sich ferner einen dem $E^{2}$ ähnlichen und ähnlich liegenden Kejelschnitt $E_{1}^{2}$ von doppelt so gro/sen Dimensionen, dessen Mittelpunct $E_{1}$ in der Curve $C_{1}^{3}$ liegt, und bewegt diesen Kegelschnitt $E_{1}^{2}$ so, dafs während sein Mittelpunct $E_{1}$ die ganze Curve $C_{1}^{3}$ durchläufl, er stets mit $E^{2}$ ähnlich liegend ist, oder seine Axen stets sich selbst parallel bleiben, so wird die gegebene Curve $C^{3}$, im Allgemeinen, 42 mal von dem auf diese Weise bewegten Kegelschnitle $E_{1}^{2}$ berïhrt."

IV. In dem Vorstehenden kamen beiläufig solche einzelne Sehnen $\boldsymbol{S}_{10}$, $\mathfrak{S}_{1}$ und $\mathfrak{S}_{1}$ vor, bei welchen die Tangenten in ibren Endpuncten an die Basis $\boldsymbol{C}^{3}$ parallel waren, und zwar kamen $6 \mathfrak{S}_{0}$ (II. 1.), $24 \mathfrak{S}_{0}$ (II. 3.) und $18 \mathfrak{S}_{1}$ (III.) in Betracht. Fassen wir diese Eigenschaft für sich auf und bezeichnen jede Sehne, welche überhaupt die Berührungspuncte, $\mathfrak{a}$ und $\mathfrak{a}_{1}$, irgend zweier paralleler Tangenten, $\mathfrak{A}$ und $\mathfrak{A}_{1}$, der Basis $\boldsymbol{C}^{3}$ verbindet, durch $\mathfrak{S}$, so ergeben sich folgende Resultate.

„Alle Sehnen S, welche die Berührungspuncte je zueier paralleler Tangenten der gegebenen $B$ asis $C^{3}$ verbinden, berühren eine Curve

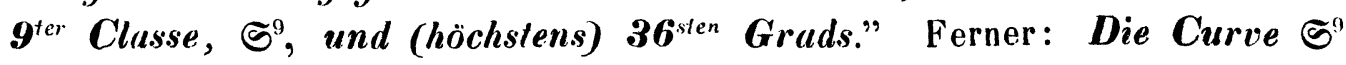
hat 6 dreifuche Tangenten, $\mathfrak{\subseteq}_{3}$, welche sich parureise in den oben genannten, in der Curve $\boldsymbol{E}^{2}$ liegenden drei Punclen $\boldsymbol{X}, \boldsymbol{Y}, \boldsymbol{Z}$ (III.) schneiden, und welche nebstdem zu je 3 durch 4 Puncte $q, r, s$ und $t$ gehen, so da/s sie die 6 Seiten eines vollständigen Vierecks rqst sind. Dieselbe Curve berührt auch die $3 A_{s}$ der $C^{3}$, und swar jede im Mittelpuncte derjenigen Hyperbel, welche die $C^{3}$ in ihrem Berührungspuncte $a_{\infty}$ (II. 2.) mit der jedesmaligen $A_{s}$ füfpunctig berührt. Wird jede Tangente der $C^{3}$, welche mit einer ihrer $3 \boldsymbol{A}_{s}$ parallel ist, durch $\mathfrak{A}_{0}$ und ihr Berührungspunct durch $\mathfrak{a}_{0}$ bezeichnet, so giebt es im Ganzen $12 \mathfrak{A}_{0}$ und $12 \mathfrak{a}_{0}$. Diese 12 Tangenten $\mathfrak{A}_{1}$ sind zugleich besondere Sehnen $\mathfrak{S}$ und herühren die Curve $\mathfrak{S}^{9}$ 
44 2. Stciner, algeb. Curven, welche Mittelp. haben, u. Eigens. allgem. algeb. Curven.

in den nämlichen Puncten $\mathfrak{a}_{10}$. Wird ferner jede Tangente der $\boldsymbol{C}^{3}$, welche mit einer ihrer $9 \mathfrak{W}$ parallel ist, durch $\mathfrak{B}$ und ihr Berührungspunct durch $\mathfrak{v}$ bezeichnet, so giebt es im Ganzen 36 Puncte $\mathfrak{y}$, und somit auch 36 Sehnen $\mathfrak{w} \mathfrak{v}=\mathfrak{S}$, welche die besondere Eigenschaft haben, dafs sie die Curve $\mathfrak{S}^{9}$ yerade in den Puncten $\mathfrak{v}$ berühren; zudem berühren auch die $9 \mathfrak{W}$ selbst, als specielle Sehnen $\mathfrak{S}$, die Curve $\mathfrak{S}^{9}$ in den zugehörigen Puncten $\mathfrak{w}$. Hieraus und aus Früherem ergeben sich folgende Beziehungen der Curve $\mathfrak{S}^{9}$ zu den Curven $\boldsymbol{C}^{3}$ und $\boldsymbol{S}_{1}^{6}$.

„Die Curve $\mathfrak{S}^{9}$ berührl die Basis $\boldsymbol{C}^{3}$ in ihren 9 Wendepunclen $\mathfrak{w}$, so wie in den 12 Puncten $\mathfrak{a}_{0}$. Die $5 \pm$ yemeinschafliche Tangenten beider Curren bestehen: 1) aus den 9 Wendetangenten $\mathfrak{X}$ der $C^{3}$, jede dreifach gezählt, 2) aus den 12 Tangenten $\mathfrak{A}_{0}$, jede doppelt gezählt, und 3) uus den 3 Asymplolen $\boldsymbol{A}_{s}$ der $C^{3}$; was zusammen 54 ausmacht."

„Die 54 gemeinschuftichen Tangenten der Curven $\mathcal{S}^{9}$ und $\boldsymbol{S}_{1}^{6}$ bestehen: 1) aus den $9 \mathfrak{W}$ und $3 A_{s}$ der Basis $C^{3}$, jede doppelt gezähll, 2) aus den obigen $24 \mathfrak{S}_{0}$ (II. 3.), und 3) aus den $6 S_{0}$ (II. 1.); was zusammen 54 betrügt."

Von den gemeinschaftlichen Puncten der Curven $\mathcal{S}^{9}$ und $C^{3}$ kennen wir bereits 78, nämlich die $9 \mathfrak{w}$ und $12 \mathfrak{a}_{0}$, doppelt gezählt, und die $36 \mathfrak{y}$. Wofern nun der Grad der Curve $\mathfrak{S}^{9}$ nicht geringer als 36 ist (was ich jedoch noch nicht genügend bewiesen habe), so fehlen noch 30 Puncte; jeder derselben ist der drilte Schnitt irgend einer Sehne $\mathfrak{a a}_{1}(=\mathbb{S})$ mit der. Basis $C^{3}$, und zugleich der Berührungspunct dieser Sehne mit der Curve $\mathfrak{S}^{9}$. Aus der Lage jener 78 Puncte schliefst man: da/s durch diese 30 Puncte Curven $10^{\text {ten }}$ Gruds gehen körnen.

„Der Berührungspunct, etwa $\mathfrak{s}$, jeder beliebigen Sehne $\mathfrak{a}_{1}=\mathfrak{S}$ mit ihrer Orlscurve $\mathfrak{S}^{9}$ ist einfach dadurch bestimmt, dafs er mit den beiden Krümmnungs-.Mitlelpunclen, etwa $m$ und $m_{1}$, der Basis $C^{3}$ in den Endpuncten a und $\mathfrak{a}_{1}$ der Selıne in einer Geraden liegl, so da/s die Gerude inm, allemal die Sehne $\mathfrak{a a}_{1}$ im verlangten Berührungspuncte s schneidet." Daraus schliefst man, unter andern:

„Du/s es in einer beliebigen Curve dritlen Grads $C^{3}$, im Allgemeinen, 36 Para parallele gleiche und gleichliegende Krüınnungsradien giebt."

Wird die Mitte jeder Sehne $\mathfrak{a \mathfrak { a }}_{1}=\mathfrak{S}$ durch $\mathfrak{M}$ bezeichnel, so folgt weiter: „Der Orl der Mitlen $\mathfrak{M}$ aller Sehnen $\mathfrak{G}$, welche die Berülırungspuncte paralleler Tangenten der gegebenen Basis $C^{3}$ verbinden, ist eine 
Curve 12ten Grads, $\mathfrak{M}^{12}$, und $\mathbf{9 6}^{\text {ster }}$ Classe, welche die Basis $C^{3}$ in ihren 9 Wendepuncten $\mathfrak{w}$ berülirt, in den 6 Puncten $\boldsymbol{P}_{0}$ schneidet, und ilhre drei unendlich entfernten Puncte $a_{\infty} z u$ vierfachen Puncten hat; was zusammen die volle Zuhl, $=36$, gemeinschaftliche Puncte beider Curven ausmachl." Die 3 mal 4 Asymptoten der Curve $\mathfrak{M}^{12}$, welche beziehlich den $3 \boldsymbol{A}_{s}$ der $\boldsymbol{C}^{3}$ parallel, liegen respective in der Mitte zwischen jeder $\boldsymbol{A}_{s}$ und den mit ihr parallelen 4 Tangenten $\mathfrak{A}_{1}$.

„Die Curve $\mathfrak{M}^{12}$ schneidet die Curve $\mathbb{E}^{2}$ in den nümlichen 6 Puncten $\boldsymbol{P}_{0}$ und nebstdem in den 18 Puncten $P_{1}$ (III.)."

Wenn man die Mitte $\mathfrak{M}$ irgend einer Sehne $\mathfrak{a}_{1}=\mathfrak{S}$ als Pol $\boldsymbol{P}$ annimml, so berührt dessen innere Polare $J^{2}$ die Basis $C^{3}$ in den Endpuncten $\mathfrak{a}$ und $\mathfrak{a}_{1}$ der Sehne. Für keinen andern. Pol können şich $\boldsymbol{J}^{2}$ und $\boldsymbol{C}^{3}$ berühren, d. h. sie können sich nicht blos in einem Puncte oder nur einmal berühren, sondern sobald sie sich in irgend einem Puncte, $\mathfrak{a}$, einfach berühren, so berühren sie einander nothwendig noch in einem andern Puncte, $\mathfrak{a}_{1}$, und alsdann sind die zugehörigen Berührungstangenten, $\mathfrak{A}$ und $\mathfrak{A}_{1}$, parallel, die Berührungssehne $\mathfrak{a a n}_{1}$ geht durch den jedesmaligen Pol $\boldsymbol{P}$ und wird durch ihn gehälftet. Also:

„Der Orl des Pols, dessen innere Polare $J^{2}$ die Basis $C^{3}$ berühren soll, ist die näınliche obige Curve $12^{\text {ten }}$ Grads $\mathfrak{M}^{12}$; dabei berühren sich die Curven $J^{2}$ und $C^{3}$ zugleich in zwei Puncten und die zugehörigen beiden.Berülurungstangenten sind stets parallel." Daraus folgt weiter: „Du/s es in der gegebenen Basis $C^{3}$ keine zwei Sehnen 5 geben kann, welche einander hälften; und daher kann auch die Curve $\mathfrak{M}^{12}$, au/ser jenen drei vierfachen Puncten $a_{\infty}$, keinen andern vielfachen Punct haben."

\section{\$. 16.}

Die im Vorstehenden (\$. 15.) über die allgemeine Curve dritten Grads aufgestellten Sätze und Eigenschaften erleiden mehr oder weniger erhebliche Modificationen, wenn die Curve von specieller Art ist. Die wesentlichsten besondern Arten sind etwa folgende:

I. Wenn die Curve $C^{3}$ einen Doppelpunct hat; wobei auch noch die dreifache Art des Doppelpuncts zu berücksichtigen ist (\$. 11.).

II. Wenn die Curve zwei Doppelpuncte hat, oder in einen Kegelschnitt und eine Gerade zerfällt.

III. Wenn die Curve drei Doppelpuncte hat, oder in drei Gerade zerfällt. 
46 2. Steiner, algeb. Curven, welche Mittelp. haben, u. Eigens. allgem. algeb. Curven.

Wiewohl diese Fälle zu mehren nicht uninteressanten besondern Sätzen führen, so mufs ich hier doch die nähere Discușsion derselben unterlassen.

\section{\$. 17.}

Ist nun ferner die gegebene Basis eine allgemeine Curve vierten Grads, $\boldsymbol{C}^{4}$, und somit die innere Polare jedes Pols eine Curve dritten Grads, $\boldsymbol{J}^{3}$, so kann letztere möglicherweise nur entweder in eine Curve zweiten Grads und in eine Gerade, oder in drei Gerade zerfallen; und zwar kann dieses Zerfällen nur dadurch geschehen, dafs von den durch den jedesmaligen Pol $\boldsymbol{P}$ gehenden 6 Sehnen $S$, oder $a a_{1}, b b_{1}, c c_{1}, d d_{1}, e e_{1}$ und $f f_{1}$, irgend zwei aufeinanderfallen, und eine Doppelsehne $\boldsymbol{S}_{2}$ bilden; denn da alsdann durch die in $\boldsymbol{S}_{2}$ liegenden zw̧ei Paar Endpuncte, etwa $a$ und $a_{1}, b$ und $b_{1}$, keine eigentliche Curve $\boldsymbol{J}^{3}$ gehen kann, so mufs sie zerfallen, und zwar mufs $\boldsymbol{S}_{2}$ selbst ein Bestandtheil von ihr sein. Der andere Bestandtheil geht dann durch die 8 Endpuncte der noch übrigen 4 Sehnen $\dot{S}$ und ist, im Allgemeinen, irgend ein Kegelschnitt $\boldsymbol{J}^{2}$, der den Pol $\boldsymbol{P}$ zum Mittelpunct hat, aber welcher jedoch in besondern Fällen auch selbst noch in zwei Gerade zerfallen kann, und zwar auf zwei Arten. Nämlich 1) sobald es sich ereignet, dafs von den übrigen 4 Sehnen auch noch ein Paar aufeinanderfällt, so fällt nothwendigerweise auch noch das dritte Paar aufeinander, so dafs dann $\boldsymbol{J}^{3}$ aus drei Doppelsehnen $\boldsymbol{S}_{2}$ besteht; oder 2) kann sich ereignen, dafs von den Endpuncten der übrigen 4 Sehnen $c c_{1}, d d_{1}, e e_{1}$ und $/ f_{1}$ irgend vier, jedoch von jeder Sehne einer, wie etwa $c, d, e$ und $f$; in einer Geraden $J$ liegen, so liegen nothwendigerweise die vier andern $c_{1}, d_{1}, e_{1}$ und $f_{1}$, in einer andern Geraden $\boldsymbol{J}_{1}$, so dafs dann $\boldsymbol{J}^{3}$ aus drei Geraden $\boldsymbol{S}_{2}, \boldsymbol{J}$ und $\boldsymbol{J}_{1}$ besteht, wovon die zwei letztern parallel sind und gleich weit vom Pol $\boldsymbol{P}$ abstehen. Demnach kann die innere Polare $\boldsymbol{J}^{3}$ möglicherweise bestehen:

A. Aus $\boldsymbol{J}^{2}+\boldsymbol{S}_{2}$, d. h. aus einem Kegelschnitte $\boldsymbol{J}^{2}$ und einer durch dessen Mittelpunct $\boldsymbol{P}$ gehenden Geraden oder Doppelsehne $\boldsymbol{S}_{2}$.

- B. a) Aus $3 \boldsymbol{S}_{2}$, d. h. aus drei durch deñ Pol $\boldsymbol{P}$ gehenden Doppelsehnen $S_{2}$; oder

b) Aus $\boldsymbol{S}_{2}+\boldsymbol{J}+\boldsymbol{J}_{1}$, d. h. aus einer durch den Pol $\boldsymbol{P}$ gehenden Doppelsehne $\boldsymbol{S}_{2}$ und aus zwei gleich weit von demselben abliegenden parallelen Geraden $\boldsymbol{J}$ und $\boldsymbol{J}_{1}$.

Der Fall $(\boldsymbol{A}$.) kommt am häufigsten vor, wogegen die Fälle unter $(\boldsymbol{B}$.) nur für einzelne bestimmte Pole eintreten. Nämlich Fall (B. a.): Es giebt 
iim Ganzen nur 9 solche Pole, die $\boldsymbol{P}_{3}$ heifsen sollen, für welche die innere Polare $J^{3}$ in drei Doppelsehnen $S_{2}$ zerfïllt," und zwar sind dieselben zugleich die der Geraden $G_{\infty}$ in Bezug auf die Basis entsprechenden 9 Pole, d. h. sie sind die gemeinschaftlichen Schnitıuncte aller äu/sern ersten Polaren $A^{3}$ in Bezug auf $C^{4}$, deren Pole in der Geraden $\boldsymbol{G}_{\infty}$ liegen. Die besondern einzelnen Pole, für welche der Fall $(\boldsymbol{B} . \boldsymbol{b}$.) eintritt, sind schwieriger anzugeben; wie man weiter unten sehen wird.

Über den Ort des Pols, dessen innere Polare auf die angegebene Weise in Theile zerfällt und über den Ort aller dabei vorkommenden Doppelsehnen, so wie über andere damit in Beziehung stehende Umstände, ergeben sich, unter andern, folgende Sätze und Eigenschaftèn.

„Der Ort des Pols $P$, dessen innere Polare auf die angegebene Art in Theile zerfällt, ist eine Curve 10 ${ }^{\text {ten }}$ Grads, $P^{10}$, und 36 $^{\text {ster Classe, }}$ welche die genannten besoudern 9 Pole $\boldsymbol{P}_{3} \approx \boldsymbol{z}$ dreifuchen Puncten hat, die Basis $C^{+}$in ihren 4 unendlich entfernten Puñcten $a_{\infty}$ berührt und somit deren $4 \boldsymbol{A}_{s}$ auch selbst zu Asymptoten hat." Die noch übrigen 32 gemeinschaftlichen Puncte der beiden Curven $\boldsymbol{P}^{10}$ und $\boldsymbol{C}^{4}$ sind solche besondere Pole, etwa $\boldsymbol{P}^{0}$, für welche die zugebörige Doppelsehne $\boldsymbol{S}_{2}$ in eine Tangente der Basis $\boldsymbol{C}^{4}$ übergeht, etwa $\boldsymbol{S}_{2}^{0}$, wobei nämlich das eine Paar Endpuncte, $b$ und $b_{1}$, sich zum Berührungspunct $\boldsymbol{P}^{\prime \prime}$ vereinigt hat. Also:

„Eine beliebige Curve vierlen Grads $C^{4}$ hat im Gunzen 32 solche Tangenten $\boldsymbol{S}_{2}^{\prime \prime}$, welche von ihr in zuei voin Berührungspunct $\boldsymbol{P}^{\prime \prime}$ gleichweit abstehenden Puncten a und a geschnitlen werden." *) ,Durch die 32 Berührungspuncte $\boldsymbol{P}^{\prime \prime}$ können Curven $\mathcal{B}^{\text {ten }}$ Grads gehen."

*) Dieser Satz stimmt mit demjenigen überein, welchen Herr Professor Hesse im 36sten Bande S. 161 d. Journ. zuerst aufgestellt hat; denn beide Sätze gehen durch Projection in einander über; oder beide Sätze sind zugleich in dem folgenden Salze enthalten.

"Bestimmt man in jeder Tangente einer gegebenen Curve $4^{\text {tèn }}$ Grads $C^{4}$ den ihrem. Berïhrungspuncte, in Bezug auf ihre zwei Schnittpuncte mit der Curve, zugeordmeten vierten harmonischen Punct $Q$, so ist dessen Ort cine Curve $52^{\text {sten }}$ Grads, $Q^{32}$, welche die gegebene Curve in ihren 24 Wendepuncten dreipunctig berührt (dic Wendetangenten mit ihr yemein hat) und sic in den j6 Berührungspuncten ihrer 28 Doppellangenten schneidet; was zusammen die volle Zahl gemeinschaftliche Pructe beider Curven ausmacht, $24 \cdot 3+56=128$." "Die obigen besondern 52 Tangenten $S_{2}^{0}$ sind den 52 Asymptoten der Curve $Q^{32}$ parallel." Bei dieser Gelegenheil erlaube ich mir noch folgende Bemerkung.

Die im obigen Monatsbericht durch $Q_{0}^{3(n-2)}$ bezeichnete Kerncurve ist, für die Basis $=C^{4}$, eine bestimmte Curve $6^{\text {ten }}$ Grads, $Q_{0}^{6}$, welche durch die 24 Wendepuncte der 
48 2. Steiner, algeb. Curven, welche Mittelp."haben, u. Eigens. allgem. algeb. Curven.

Von den 10 gemeinschaftlichen Puncten der Curve $\boldsymbol{P}^{10}$ und der Geraden $G_{\infty}$ kennen wir erst vier, die $4 u_{\infty}$; allein von diesen, so wie von den ihnen zugehörigen Asymptoten, $4 \boldsymbol{A}_{s}$, hången die noch übrigen 6 Puncte, so wie die Richtungen ihrer zugehörigen Asymptoten ab. Bezeichnen wir für einen Augenblick die 4 Puncte $a_{\infty}$ durch $\alpha, \beta, \gamma$ und $\delta$, und die 6 unbekannten Puncte durch $x$ und $x_{1}, y$ und $y_{1}, z$ und $z_{1}$, so ist jedes Paar der letztern immer zu den in zwei Paare geórdneten erstern zugleich harmonisch, so dafs etwa

$$
x \alpha x_{1} \beta \text { und } x \gamma x_{1} \delta ; y \alpha y_{1} \gamma \text { und } y \beta y_{1} \delta ; z \alpha z_{1} \delta \text { und } z \beta z_{1} \gamma
$$

harmonisch sind. Oder zieht man durch einen beliebigen Punct die 4 Geraden $\boldsymbol{A}, \boldsymbol{B}, \boldsymbol{C}$ und $\boldsymbol{D}$ den 4 Asymptoten $\boldsymbol{A}_{s}$ parallel, ordnet dieselben auf die möglichen drei Arten zu zwei Paaren, nämlich $\boldsymbol{A B}$ und $\boldsymbol{C D}, \boldsymbol{A C}$ und $\boldsymbol{B D}$, $\boldsymbol{A D}$ und $\boldsymbol{B C}$, und construirt zu diesen andere drei Strahlenpaare $\boldsymbol{X}$ und $\boldsymbol{X}_{1}$, $Y$ und $' Y_{1}, Z$ und $Z_{1}$ so, dafs zugleich

$X A X_{1} B$ und $X C X_{1} D ; Y A Y_{1} C$ und $Y B Y_{1} D ; Z A Z_{1} D$ und $Z B Z_{1} C$ harmonisch sind: so sind diese Strahlen $\boldsymbol{X}, \boldsymbol{X}_{\mathbf{1}} ; \boldsymbol{Y}, \boldsymbol{Y}_{\mathbf{1}} ; \boldsymbol{Z}, \boldsymbol{Z}_{\mathbf{1}}$ den unbekannten 6 Asymptoten der Curve $P^{10}$ parallel, und somit nach jenen 6 Punclen $x, x_{1}$; $y, y_{1} ; z, z_{1}$ gerichtel, welche die Curve mit $\boldsymbol{G}_{\infty}$ gemein hat. Von diesen drei Punctenpaaren sind immer zwei Paar reell und das dritte imaginär, und dein entsprechend sind auch von den 6 Asymptoten 4 reell und 2 imaginär, wofern nämlich jene ersten 4 Asymptoten $\boldsymbol{A}_{s}$ reell sind.

Die Curve $\boldsymbol{P}^{10}$ geht ferner insbesondere auch durch die Mitten der 28 Doppeltangenten der Basis $C^{4}$.

Was nun weiter die Doppelsehnen, $\boldsymbol{S}_{2}$, betrifft, so ist zwar die Curve $\boldsymbol{P}^{\prime \prime}$ der Ort ihrer Milten, $\boldsymbol{P}$, aber die Sehnen selbst umhüllen eine andere Curve, nämlich :

Basis $C^{4}$ geht. Da nun durch dieselben 128 Puncte, welche die Curve $Q^{32}$ mit $C^{4}$ gemein hat, unendlich viele andere Curven $32^{\text {sten }}$ Grads gehen, und da, wenn eine niedrigere Curve $Q^{x}, x<32$, durch $4 x$ der genannten 128 Puncte geht, dann eben so durch die noch äbrigen $128-4 x=4(32-x)$ Puncte unzählige Curven $(32-x)^{\text {ten }}$ Grads, $Q^{32-x}$, gehen können, so folgt also: "Da/s durch die 36 Bcriihrungspuncte der 28 Doppeltangenten der Basis $C^{4}$ unzählige Curven 14ten Grads, $Q^{14}$, gehen lï̈nnen." Denn denkt man sich die Kerncurve $Q_{0}^{6}$ dreifach (oder nach Rechnungsart: ihre Gleichung in Cubus erhoben), so ist sie als oine Curve $18^{\text {ten }} \mathrm{Grads}, Q_{0}^{18}$, anzusehen, und als solche geht sie durch die 24.3 oder 4.18 Puncte, welche $Q^{32}$ und $C^{4}$ in den 24 Wendepuncten gemein haben, und folglich können durch die 56 Berührungspuncte der 28 Doppeltangenten unzählige Curven $Q^{14}$ gehen. - Auf wie mannichfaltige Weise solche Curve $Q^{14}$ selbst wieder in Theile zerfallen kann, werde ich an einem geeignetern Orte nachweisen. 
2. St einer, alyeb. Curven, welche Mittelp. haben, u. Eigens. allgem. algeb.Curven.

Der Ort aller Doppelsehnen $\boldsymbol{S}_{2}$, welche Bestandtheile der zerfallenden innern Polaren $J^{3}$, oder welche in der gegebenen Basis überhaupt möglich sind, ist eine Curve $9^{\text {ter }}$ Classe, $S_{2}^{9}$, und $34^{\text {sten }}$ Grads, welche die Basis in ihren im Unendlichen liegenden 4 Puncten $a_{\infty}$ vierpunclig berührt, somit deren $4 \boldsymbol{A}_{s}$ ebenfalls zu Asymptoten hat, aber jede derselben noch in einem bestimmten andern Puncte berührt, also dieselben .zu Doppeltangenten hat; ferner berühtht die.Curve auch noch die 28 Doppellangenten der Basis und hat die Gerade $G_{\infty}$ zur sechsfachen Tangente, und zwar berührt sie diese in den nämlichen, vorhin näher bestimmten 6 Puncten $x$ und $x_{1}, y$ und $y_{1}, z$ und $z_{1}$." Nämlich denkt man sich den Pol $\boldsymbol{P}$ in einem dieser 6 Puncte, etwa in $x$, so fält die ihm zugehörige Doppelsehne $\boldsymbol{S}_{2}$ auf die Gerade $\boldsymbol{G}_{\infty}$ und berührt die Curve im conjugirten Puncte $x_{1}$, und auch umgekehrt; und eben so verhält es sich mit den beiden andern Punctenpaaren. Diese Berührungen sind mit den respectiven Puncten gleichzeitig reell oder imaginär.

Da die Basis $C^{4}$, im Allgemeinen, von der $12^{\text {ten }}$ Classe ist, so hat sie mit der Curve $S_{2}^{9}$ im Ganzen 12.9 $=108$ Tangenten gemein, und diese bestehen: 1) in den obigen 32 Tangenten $S_{2}^{(0} ; 2$ ) in den 28 Doppeltangenten der Basis, jede doppelt gezählt; und 3 ) in den $4 \boldsymbol{A}_{s}$, jede fünffach gezählt; was zusammen richtig $32+28 \cdot 2+4 \cdot 5=108$ ausmacht.

Von den gemeinschaftlichen Puncten der Curve $\boldsymbol{S}_{2}^{9}$ und der Geraden $\boldsymbol{G}_{\infty}$ kennen wir bereits 16 , nämlich die 6 Berührungspuncté $x, x_{1}, y, y_{1}, z$ und $z_{1}$, jeder doppelt gezählt, und die 4 Puncte $a_{\infty}$. Da die Curve vom $34^{\text {sten }}$ Grad ist, so fehlen also noch 18 Puncte, welche durch folgende Betrachtung näher bestimmt werden, aus der zugleich noch einige andere Eigenschaften hervorgehen.

Durch jeden gegebenen Punct gehen, im Allgemeinen, 9 Doppelsehnen $\boldsymbol{S}_{2}$. Liegt der Punct in der Basis $\boldsymbol{C}^{4}$ selbst, er heifse $a$, so ist er ein Endpunct von jeder der $9 \boldsymbol{S}_{2}$ und alsdann liegen die ihm sugehörigen andern 9 Endpuncle $a_{1}$ in einer Curve $3^{\text {ten }}$ Grads, $a_{1}^{3}$, welche die Basis in Puncte a dreipunctig berührt; und ebenso liegen die Mitten $P$ der $\mathbf{9} S_{2}$ in einer andern Curve $3^{\text {ten }}$ Grads, $\boldsymbol{P}^{3}$, welche die Basis im Puncte a zweipunctig berührt. Ist insbesondere der Punct a ein Wendepunct der Basis, so ist er dasselbe auch von jeder der beiden Curven $a_{1}^{3}$ und $\boldsymbol{P}^{3}$. Und ist $a$ einer der obigen 32 Schnittpuncte $P^{0}$ der Curven $\boldsymbol{P}^{10}$ und $C^{4}$, so wird die Basis in illm von der Curve $a_{1}^{3}$ vierpunclig und von der Curve $\boldsymbol{P}^{3}$

Crelle's Journal f. d. M. Bd. XLVII. Heft 1. 
50 2. St einer, algeb.Curven, welche Mittelp. haben, u. Eigens. allgem. algeb.Curven.

dreipunctig berührt, so da/s diese beiden Curven einander daselbst auch dreipunctig berühren.

Wiewohl durch jeden Punct 9 Doppelsehnen gehen, so sind dieselben doch nur zu 3 und 3 parallel, so dafs es nach jeder gegebenen Richtung nur je $3 S_{2}$ giebt, oder mit andern Worten: durch jeden Punct $\boldsymbol{Q}$ in der Geraden $\boldsymbol{G}_{\infty}$ gehen nur 3 (nicht selbst im Unendlichen liegende) Doppelsehnèn $\boldsymbol{S}_{2}$, indem die 6 übrigen auf die Gerade $\boldsymbol{G}_{\infty}$ selbst fallen. Die Mitten, $\boldsymbol{P}$, je dreier paralleler Doppelsehnen liegen nothwendigerweise in einem Durchmesser, $\boldsymbol{D}$, der Basis $C^{4}$ (\$. 15. I.), oder, was dasselbe ist, in der dritten aufsern Polare, $\boldsymbol{D}$, des Punctes $\boldsymbol{Q}$ in Bezug auf die Basis, und die drei Sehnen $\boldsymbol{S}_{2}$ haben die dem Durchmesser $\boldsymbol{D}$ conjugirle Richtung. Man denke sich ferner von demselben Puncte $\boldsymbol{Q}$ die erste äufsere Polare $\boldsymbol{A}^{3}$ in Bezug auf die Basis, so geht dieselbe, wie schon bemerkt, durch jene 9 Pole $\boldsymbol{P}_{3}$, welche dreifache Puncte der Curve $\boldsymbol{P}^{10}$ sind, und daher kann sie die letztern aufserdem nur noch in irgend drei Puncten $\boldsymbol{P}$ schneiden, welche (vermöge der Lage der $9 \boldsymbol{P}_{3}$ ) nothwendig zugleich in irgend einer Geraden liegen müssen. Diese Gerade ist aber gerade der genannte Durchmesser $\boldsymbol{D}$ und die drei Schnittpuncte $\boldsymbol{P}$ sind gerade die Mitten jener nach $\boldsymbol{Q}$ gerichteten $3 \boldsymbol{S}_{2}$. Also:

„,Denkt man sich von irgend einem Puncte $\boldsymbol{Q}$ in der Geraden $\boldsymbol{G}_{\infty}$ die erste und dritte äu/sere Polare in Bezug auf die Basis $C^{4}, A^{3}$ und $\boldsymbol{D}$, so schneiden sich dieselben in denjenigen 3 Polen $\boldsymbol{P}$, deren zugehörige 3 Doppelsehnen $\boldsymbol{S}_{2}$ nach dem nümlichen Puncte $\boldsymbol{Q}$ gerichtet sind, oder welche die dem Durchmesser $\boldsymbol{D}$ conjugirte Richtung haben." Und: Beweyt sich der Punct $Q$ längs der Geraden $G_{\infty}$, so ist der Ort der 3 Schnitte $P$ seiner ersten und dritten äufsern Polare die nämliche Curve $1^{\text {ten }}$ Grads $P^{11}$, welche, alle Pole enthält, deren innere Polaren $J^{3}$ zerfallen." Alle bei dieser Bewegung vorkommende Polaren $A^{3}$ bilden einen Curvenbüschel, $\boldsymbol{B}\left(\boldsymbol{A}^{3}\right)$, mit den 9 Grundpuncten $\boldsymbol{P}_{3}$.

Nun kann sich ereignen, dafs von den genannten Polaren $\boldsymbol{A}^{3}$ irgend eine die Curve $\boldsymbol{P}^{10}$ berührt, wobei von den 3 Schnitlpuncten $\boldsymbol{P}$ zwei sich zu einem Berührungspuncte, etwa $\mathfrak{P}_{10}$, von $\boldsymbol{P}^{10}, \boldsymbol{A}^{3}$ und $\boldsymbol{D}$ vereinigen, und wobei also auch die zugehörigen beiden Doppelsehnen $\boldsymbol{S}_{2}$ in eine, etwa $\boldsymbol{S}_{2}^{\prime \prime}$, zusammenfallen; alsdann berührt diese Sehne $\mathfrak{S}_{2}^{\prime \prime}$ die Curve $\boldsymbol{S}_{2}^{9}$ im entsprechenden Puncte $\boldsymbol{Q}$, oder für diesen Fall $\boldsymbol{Q}_{0}$, und ist somit eine Asymptote derselben, so wie $\boldsymbol{Q}_{0}$ einer ihrer, oben verlangten, gemeinschaftlichen Puncte mit der 
Geraden $\boldsymbol{G}_{\infty}$ ist. Da nun eine Curve $\boldsymbol{P}^{p}$ von den Curven eines Büschels $\boldsymbol{B}\left(\boldsymbol{Q}^{q}\right)$ in $\boldsymbol{p}(\boldsymbol{p}+2 q-3)$ Puncten berührt werden kann (s. obigen Monatsbericht): so müfste danach die Curve $\boldsymbol{P}^{10}$ von dem Büschel Polaren $\boldsymbol{B}\left(\boldsymbol{A}^{3}\right)$ in $10(10+2 \cdot 3-3)=130$ Puncten $\mathfrak{P}_{0}$ berührt werden. Allein von diesen 130 Puncten werden 108 durch jene gemeinschaftlichen 9 Puncte $\boldsymbol{P}_{3}$ absorbirt, so dafs nur noch 22 frei bleiben, unter welchen sich jedoch noch jene bereits bekannten 4 Puncte $a_{s}$ befinden, so dafs es also nur 18 zuläfsige Berührungspuncte $\mathfrak{P}_{0}$ giebt: und diesen 18 Puncten $\mathfrak{P}_{0}$ entsprechen somit auf der Geraden $\boldsymbol{G}_{\infty}$ die verlangten 18 Puncte $\boldsymbol{Q}_{0}$, so wie die zugehörigen 18 Asymptoten $\mathfrak{S}_{2}^{0}$ der Curve $\boldsymbol{S}_{2}^{9}$. Das heifst: Die oben noch fehlenden 18 gemeinschaftichen Puncte $\boldsymbol{Q}_{11}$ der Curve $\boldsymbol{S}_{2}^{9}$ und der Geraden $\boldsymbol{G}_{\infty}$ haben die Eigenschaft, oder sind dadurch bestimmt, da/s die erste und dritte Polare eines jeden derselben in Besug auf die Basis sich in irgend einem Puncte $\mathfrak{P}_{0}$ berühren, und da/s die jenem Puncte zugehörige Asymptote $\mathfrak{S}_{2}^{\prime \prime}$ zugleich durch den letatern Punct geht. Dieselbe Eigenschaft besitzen übrigens auch jene 4 Puncte $a_{s}$, jedoch mit dem Unterschiede, dafs jeder $\boldsymbol{Q}_{0}$ und $\mathfrak{P}_{11}$ zugleich ist, d. h. dafs die erste und dritte Polare eines jeden sich mit der Curve $\boldsymbol{P}^{10}$ in ihm selbst berühren, und zwar ist seine dritte Polare die zugehörige Asymptole $\boldsymbol{A}_{s}$ der Basis, so dafs also die 4 Asymptoten der Basis zugleich specielle Durchmesser derselben sind. Die 18 Asymptoten $\mathfrak{S}_{2}^{\prime \prime}$ haben als Doppelsehnen die besondere Eigenschaft: da/s die in ihren Endpuncten $a$ und $a_{1}, b$ und $b_{1}$ an die Basis $C^{4}$ gelegten Tangenten-Paare $A$ und $A_{1}$, $B$ und $B_{1}$ sich auf dem zugehörigen Durchmesser $D$ schneiden, so da/s dieser Durchmesser eine Diagonale des vollständigen Vierseits $\boldsymbol{A A}_{1} \boldsymbol{B B}_{1}$ ist, dessen beide andern Diagonılen mit $\mathfrak{S}_{2}^{0}$ parallel sind.

Jeder Durchmesser $\boldsymbol{D}$ schneidet die Curve $\boldsymbol{P}^{10}$ aufser jenen 3 Puncten $\boldsymbol{P}$, die zugleich in der entsprechenden Polare $\boldsymbol{A}^{3}$ liegen, in noch 7 andern Puncten $\boldsymbol{P}$; aber jene unterscheiden sich von diesen wesentlich dadurch, dafs die ihnen zugehörigen Doppelsehnen $\boldsymbol{S}_{2}$ die dem Durchmesser conjugirte Richtung haben, wogegen die zu den 7 andern gehörigen Doppelsehnen zu je einem andern Durchmesser conjugirt sind. Also: Von den je 10 Polen $P$, welche in irgend einem Durchmesser $D$ liegen, gehören ihm 3 in der Art eigenthümlich an, da/s die ihnen zugehörigen Doppelsehnen die dern Durchmesser conjugirte Richtung haben, oder nach seinem in $G_{\infty}$ liegenden Pol $Q$ gerichtet sind.

Über die Durchmesser insgesammt hat man folgenden Satz: 
52 2. St ein er, algeb.Curven, welche Mittelp. haben, u. Eigens. allgem. algeb.Curven.

„Alle Durchmesser, $\boldsymbol{D}$, der gegebenen Basis $\boldsymbol{C}^{4}$ umhüllen eine bestimmte Curve $3^{\text {ter }}$ Classe, $D^{3}$, und $4^{\text {ten }}$ Grads, welche drei Rückkehrpuncte, $\mathfrak{r}$, und eine Doppeltangente, $\mathrm{D}_{2}$, hat; und namentlich berührt diese Curve jede der 4 Asymptoten $\boldsymbol{A}_{s}$ der Basis (als specielle Durchmesser) in demjenigen Puncte, welcher der Schwerpunct von ihren 3 Schniltpuncten mit den 3 andern Asymptoten ist." Die Curve $D^{3}$ heifst auch die dritte Polare der Geraden $G_{\infty}$ in Bezug auf die Basis $C^{4}$ (Monatsbericht).

Danach gehen also durch jeden beliebigen Punct $\boldsymbol{R}$ in der Ebene, im Allgemeinen, je drei Durchmesser der $\boldsymbol{C}^{4}$; somit auch durch jeden Punct $\boldsymbol{Q}$, in $\boldsymbol{G}_{\infty}$, drei parallele Durchmesser, etwa $\boldsymbol{D}_{q}$, und zwar haben diese die conjugirte Richtung desjenigen Durchmessers $\boldsymbol{D}$, welcher dem Puncte $\boldsymbol{Q}$ entspricht (dessen $3^{\text {te }}$ Polare ist); aber die den drei Durchmessern $\boldsymbol{D}_{q}$ conjugirten Richtungen sind unter sich, so wie auch, im Allgemeinen, von der Richtung des Durchmessers $\boldsymbol{D}$ verschieden. Nämlich:

„Die Basis $C^{4}$ hal im Ganzen nur drei Paar conjugirte Durchmesser, d. h. solche Durchmesser, wovon jeder die conjugirte Richtung des andern hat, und zwar sind dieselben beziehlich nach den obigen Puncten-Paaren $x$ und $x_{1}, y$ und $y_{1}, z$ und $z_{1}$ in der Geraden $G_{\infty}$. gerichtet, und somit den dort construirten Strahlen-Paaren $X$ und $X_{1}$, $\boldsymbol{Y}$ und $\boldsymbol{Y}_{1}, \boldsymbol{Z}$ und $\boldsymbol{Z}_{1}$ parallel. Denkt man sich den Punct $\boldsymbol{Q}$ in einem der 6 Puncte, etwa in $x$, so geht der ihm entsprechende Durchmesser $\boldsymbol{D}$ durch den conjugirten Punct $x_{1}$, und auch umgekehrt; und zwar ist dabei $x_{1}$ zugleich einer der drei Puncte $\boldsymbol{P}$, die dem Durchmesser $\boldsymbol{D}$ eigenthümlich zugehören, oder in denen er von der entsprechenden Polare $\boldsymbol{A}^{3}$ geschnitten wird.

Die 4 Asymptoten $A_{s}$ sind diejenigen besondern Durchmesser, welchen ihre eigene Richtung conjugirt ist.

Die Doppeltangente $\mathrm{D}_{2}$ der Curve $\mathrm{D}^{3}$ ist gewisserma/sen ein doppelter Durchmesser, $d$. h. ein solcher, welchem swei verschiedene Richtungen conjugirt sind, so da/s ihm auch zwei verschiedene Pole auf der Geraden $G_{\infty}$ entsprechen, etwa $Q_{2}$ und $Q_{2}^{1}$, welche nach den beiden Richtungen hin liegen; ebenso müssen ihm zweimal 3 Pole $\boldsymbol{P}$ eigenthümlich angehören und die zu denselben gehörigen Doppelsehnen $\mathbb{S}_{2}$ müssen zu 3 und 3 die conjugirten Richtungen haben, also parallel oder nach den Puncten $Q_{2}$ und $Q_{2}^{1}$ gerichtet sein.

Die conjugirten Richtungen der drei Durchmesser, welche durch 
2. St ein er, algeb. Curven, welche Mittelp. haben, u. Eigens. allgem. algeb. Curven. 53 irgend einen gegebenen Punct $\boldsymbol{R}$ gehen, sind allemal durch die Asymptoten der beiden ersten Polaren $A^{3}$ und $J^{3}$ des nünlichen Punctes bestimmt, und auch umgekehrt.

Jeder (in $\boldsymbol{P}^{10}$ liegende) Pol $\boldsymbol{P}$ gehört, im Allgemeinen, nur einem der durch ihn gehenden drei Durchmessern eigenthümlich an, nämlich demjenigen, welchem die zugehörige Doppelsehne $\boldsymbol{S}_{2}$ conjugirt ist. Nur von jenen besondern 9 Polen $\boldsymbol{P}_{3}$ gehört jeder allen drei Durchmessern zugleich an, indem ihm auch drei Doppelsehnen zugehören, welche den Durchmessern beziehlich conjugirt sind.

„Die durch jeden der 9 Pole $\boldsymbol{P}_{3}$ gehenden drei Durchmesser berühren die durch denselben gehenden drei Zweige der Curve $\boldsymbol{P}^{10}$ daselbst."

Ist der Pol $\boldsymbol{P}_{x}(=\boldsymbol{P})$ insbesondere einer der 40 gemeinschaftlichen Puncte der Curven $\boldsymbol{P}^{10}$ und $\boldsymbol{D}^{3}$, so fallen von den durch ihn gehenden drei Durchmessern zwei zusammen, nämlich auf die Tangente der Curve $D^{3}$ im Pol $\boldsymbol{P}_{x}$, welche $\boldsymbol{D}_{t}$ heifsen soll; der andere Durchmesser berührt die $\boldsymbol{D}^{3}$ in irgend einem andern Puncte, etwa $\boldsymbol{R}_{0}$, und heifse $\boldsymbol{D}_{r}$. Nun sind, hierbei zwei Fälle möglich, nämlich entweder gehört der Pol $\boldsymbol{P}_{x}$

1) dem Durchmesser $\boldsymbol{D}_{t}$, oder

2) dem Durchmesser $\boldsymbol{D}_{r}$ eigenthümlich an;

und davon hängen sodann weiter folgende interessante Umstände ab:

I. „Gehört der Pol $\boldsymbol{P}_{x}$ zum Durchmesser $\boldsymbol{D}_{t}$, so besteht seine innere Polare $J^{3}$ aus $J^{2}+\boldsymbol{S}_{2}$ und zwar ist die Doppelsehne $\boldsymbol{S}_{2}$ zugleich eine Asymptote des Kegelschnitts $\boldsymbol{J}^{2} ; "$ und

II. „Gehört der Pol $\boldsymbol{P}_{x}$ zum Durchmesser $D_{r}$, so besteht seine innere Polare aus $\boldsymbol{S}_{2}+\boldsymbol{J}+J_{1}$, wobei die Geraden $\boldsymbol{J}$ und $\boldsymbol{J}_{1}$ parallel sind und gleichweit vom Pol abstehen."

Hierbei entsteht die Frage:

Wieviele von den 40 Polen $\boldsymbol{P}_{x}$ gehören zu Durchmessern $D_{t}$, und wieviele gehören zu Durchmessern $\boldsymbol{D}_{r}$ ? oder wieviele in $\boldsymbol{J}^{2}+\boldsymbol{S}_{2}$ zerfallende innere Polaren giebt es, bei welchen $\boldsymbol{S}_{2}$ Asymptote von $\boldsymbol{J}^{2}$ ist, und wieviele giebt es, welche in drei Gerade $\boldsymbol{S}_{2}+\boldsymbol{J}+\boldsymbol{J}_{1}$ zerfallen?

Diese Frage weifs ich vor der Hand noch nicht sicher zu beantworten, und überlasse sie daher dem geneigten Leser.

Über die Curve $\boldsymbol{D}^{3}$ will ich noch Folgendes bemerken.

„Die Curve $D^{3}$ ist der Ort desjenigen Pols $\boldsymbol{R}_{0}$, dessen äu/sere Polare $A^{3}$ die Gerade $G_{\infty}$ berührt; und die dritte Polare des Berührungs- 
puncles, $\boldsymbol{Q}$, ist gerade derjenige Durchmesser $\boldsymbol{D}$, welcher die Curve $\boldsymbol{D}^{3}$ in jenem Pole $\boldsymbol{R}_{0}$ berührt." Da nun die innere Polare $\boldsymbol{J}^{3}$ desselben Pols $\boldsymbol{R}_{0}$ mit der Geraden $\boldsymbol{G}_{\infty}$ allemal die nämlichen drei Puncte gemein hat, wie die äufsere $\boldsymbol{A}^{3}$ (\$. 13. II.), so mufs auch sie die Gerade $\boldsymbol{G}_{\infty}$ in $\boldsymbol{Q}$ berühren; allein nach dem Früheren (\$.11.) ist diese Berührung nur dadurch möglich, dafs $Q$ ein Doppelpunct der Curve $\boldsymbol{J}^{3}$ ist. Daher kann man auch sagen:

„Der Ort desjenigen Pols $\boldsymbol{R}_{0}$, dessen innere Polare $J^{3}$ nur einen einzigen Doppelpunct $\boldsymbol{Q}$ (oder insbesondere auch drei Doppelpuncte) hut, ${ }^{*}$ ) ist die Curve $D^{3}$, und der Ort des Doppelpunctes ist die Gerade $G_{\infty}$." Bei denjenigen Polen $\boldsymbol{P}_{x}\left(=\boldsymbol{R}_{0}\right)$, deren innere Polaren aus $\boldsymbol{S}_{2}+\boldsymbol{J}+\boldsymbol{J}_{1}$ bestehen, und somit drei Doppelpuncte haben, liegt nur einer der letztern (der Schnitt von $\boldsymbol{J}$ und $\boldsymbol{J}_{1}$ ) auf der Geraden $\boldsymbol{G}_{\infty}$; und bei denjenigen $\boldsymbol{P}_{x}$, deren innere Polaren aus $\boldsymbol{J}^{2}+\boldsymbol{S}_{2}$ bestehen, fallen die zwei Doppelpuncte in einen zusammen, dè als ein Rückkehrpunct anzusehen ist und in $\boldsymbol{G}_{\infty}$ liegt.

„Liegt der Pol $\boldsymbol{R}_{0}$ insbesondere in einem der drei Rückkehrpuncte $\mathfrak{r}$ der Curve $D^{3}$, so ist der ihm entsprechende Punct $Q$ sugleich ein Wendepunct seiner Polare $A^{3}$ und ein Rückkelirpunct seiner Polare $J^{3}$, und zwar ist die Gerade $G_{\infty}$ beziehlich die zugehörige Wende-und Rückkehrlangente."

Liegt ein Pol $\boldsymbol{R}$ in dem Doppeldurchmesser (Doppeltangente der $\boldsymbol{D}^{3}$ ) $\boldsymbol{D}_{2}$, so gehen seine beiden Polaren $\boldsymbol{A}^{3}$ und $\boldsymbol{J}^{3}$ durch die dem $\boldsymbol{D}_{2}$ entsprechenden beiden Puncte $\boldsymbol{Q}_{2}$ und $\boldsymbol{Q}_{2}^{1}$ auf $\boldsymbol{G}_{\infty}$; und bewegt sich $\boldsymbol{R}$ längs $\boldsymbol{D}_{2}$, so bleiben also zwei Paar Asymptoten der Polaren $\boldsymbol{A}^{3}$ und $\boldsymbol{J}^{3}$ sich selbst parallel, nämlich stets nach jenen Puncten $\boldsymbol{Q}_{2}$ und $\boldsymbol{Q}_{2}^{1}$ gerichtet.

\section{\$. 18.}

Hat die Basis $C^{4}$ specielle Form, hat sie z. B. Doppel-oder Rückkehrpuncte, oder besteht sie aus Theilen, nämlich aus
1) $\boldsymbol{C}^{3}+\boldsymbol{C}^{1}$
2) $\boldsymbol{C}^{2}+\boldsymbol{C}_{1}^{2}$;
3) $\boldsymbol{C}^{2}+2 \boldsymbol{C}^{1}$
4) $4 C^{1}$ :

*) Soll die Polare $J^{3}$ zwei (und auch drei) Doppelpuncte haben, so mufs sie aus $J^{2}+S_{2}$ bestehen, somit der Ort ihres Pols die Curve $P^{10}$ sein, und dann sind die Doppelpuncte die gegenseitigen Schnitte von $J^{2}$ und $S_{2}$, etwa $\mathfrak{D}$ und $\mathfrak{D}_{1}$. Dabei kann man fragen: In welcher Curve, $\mathfrak{D}^{n}$, liegen alle diese Doppelpuncte? Ist der Grad-Exponent, $n$, et $u$ a gleich der Zuhl derjenigen Pole $\boldsymbol{P}_{x}$, welche zu Durchmessern $\boldsymbol{D}_{t}$ gehören? und sind die diesen Polen zugehörigen Doppelsehnen $\mathrm{S}_{2}$ zugleich Asympcoten der Curve $\mathfrak{D}^{n}$ ? Diese unbekannte Curve $\mathfrak{D}^{n}$ hat übrigens die 9 Pole $\boldsymbol{P}_{3}$ gleichfalls zu dreifachen Puncten, wie die Curve $P^{10}$. Der Ort der Doppelpuncte aller innern Polaren $J^{3}$ insgesammt besteht also aus $\mathfrak{D}^{n}+\boldsymbol{G}_{\infty}$. 
so werden die vorigen Sätze und Eigenschaften (\$. 17.) auf entsprechende Weise verändert, so wie auch neue Sätze herbeigeführt. Eine umständliche Erörterung aller dieser Fälle würde hier zu weit führen; daher bègnüge ich mich nur Einiges kurz anzudeuten.

I. Besteht die Basis aus $C^{2}+C_{1}^{2}$, d. h. aus irgend zwei gegebenen Curven zweiten Grads, so hat sie 4 Doppelpuncte, nämlich die gegenseitigen Schnittpuncte $q, r, s, t$ von $C^{2}$ und $C_{1}^{2}$, und es tritt zunächst die Hauptänderung ein:

„Dafs dabei die Curve $\boldsymbol{P}^{11}$ in zwei Theile $\boldsymbol{P}^{8}+\boldsymbol{P}^{2}$ und eben so die Curve $\boldsymbol{S}_{2}^{9}$ in swei Theile $\boldsymbol{S}_{2}^{6}+\boldsymbol{S}_{2}^{3}$ serfällt."

Es kann nämlich dabei die Doppelsehne $\boldsymbol{S}_{2}$ von zweierlei Art sein. Sind $a, b$ ihre Schnittpuncte mit $C^{2}$, und $a_{1}, b_{1}$ ihre Schnitte mit $C_{1}^{2}$, so können entweder

$\boldsymbol{A}$. Die Sehnen $a b$ und $a_{1} b_{1}$ dieselbe Mitte $\boldsymbol{P}$ haben, wobei dann die Wechselsehnen parweise gleich sind, $a a_{1}=b b_{1}, a b_{1}=b a_{1}$; oder

B. Die Sehnen $a b$ und $a_{1} b_{1}$ sind gleich, und ein Paar Wechselsehnen, $a a_{1}$ und $b b_{1}$ oder $a b_{1}$ und $b a_{1}$, hat dieselbe Mitte $\boldsymbol{P}$ und das andere Paar ist gleich.

Gemäfs dieser zwei Arten Doppelsehnen zerfallen die beiden Ortseurven in die genannten Theile, welche, wie folgt, gewissermafsen selbständig auftreten.

1. ,Im Falle (A.) ist der Ort des Pols $\boldsymbol{P}$ ein bestimmter Kegelschnitt $\boldsymbol{P}^{2}$, und der Ort der Doppelsehne $\boldsymbol{S}_{2}$ ist eine bestimmte Curve $\mathbf{3}^{\text {ter }}$ Classe $\boldsymbol{S}_{2}^{3}$ und $\mathbf{4}^{\text {ten }}$ Grads, welche die Gerade $\boldsymbol{G}_{\infty}$ zur Doppeltangente (und drei Rückkehrpuncte $\mathfrak{r}$ ) hat." Oder anders ausgesprochen: „,Der Ort der Transversale $\boldsymbol{S}_{2}$, welche in den zwei gegebenen Kegelschnitten $\boldsymbol{C}^{2}, \boldsymbol{C}_{1}^{2}$ solche Sehnen $a b, a_{1} b_{1}$ bildet, welche die nümliche Mitte $\boldsymbol{P}$ haben, ist eine Curve $\mathbf{3}^{\text {ter }}$ Classe $\boldsymbol{S}_{2}^{3}$ und $4^{\text {ten }}$ Grads, und der Ort der Mitte $\boldsymbol{P}$ ist eine Curve zweiten Grads $\boldsymbol{P}^{2}$." Zieht man in den gegebenen. Kegelschnitten $C^{2}$ und $C_{1}^{2}$ irgend zwei parallele Durchmesser, etwa $\alpha$ und $\alpha_{1}$, und ferner die ihnen conjugirten Durchmesser $\beta$ und $\beta_{1}$ : so treffen sich die letstern allemal in irgend einem der Pole $\boldsymbol{P}$, und die durch diesen mit den Durchmessern $\alpha$ und $\alpha_{1}$ parallel gezogene Gerade ist die ihm zugehörige Doppelsehne $\boldsymbol{S}_{2}$. Tritt der besondere Fall ein, dafs die Durchmesser $\beta$ und $\beta_{1}$ auch parallel werden: so sind alsdann $\alpha, \alpha_{1}$ der einen und $\beta, \beta_{1}$ der andern Asymptote der Curve $\boldsymbol{P}^{2}$ parullel. Zieht man durch einen beliebigen Punct 
56 2. Steiner, algeb. Curven, welche Mittelp. haben, u. Eigens. allyem. algeb. Curven.

drei Paar Gerade $\boldsymbol{A}$ und $\boldsymbol{B}, \boldsymbol{A}_{1}$ und $\boldsymbol{B}_{1}, \boldsymbol{X}$ und $\boldsymbol{X}_{1}$ beziehlich den Asymptoten der drei Kegelschnitte $\boldsymbol{C}^{2}, \boldsymbol{C}_{1}^{2}, \boldsymbol{P}^{2}$ parallel, so sind

$$
A X B X_{1} \text { und } A_{1} X_{1} X_{1}
$$

zugleich harmonisch; und wenn $\alpha$ und $\beta, \alpha_{1}$ und $\beta_{1}, x$ und $x_{1}$ die im Unendlichen liegenden Puncte derselben Kegelschnitte sind, so sind $\alpha x \beta x_{1}$ sowohl als $\alpha_{1} x \beta_{1} x_{1}$ harmonisch. - Die Curve $\boldsymbol{P}^{2}$ schneidet jede der beiden gegebenen, etwa $\boldsymbol{C}^{2}$, in denjenigen 4 Puncten $\boldsymbol{P}^{\prime \prime}$, bei welchen die zugehörige Tangente $S_{2}^{(0}\left(\right.$ an $\left.C^{2}\right)$ von der andern gegebenen Curve $C_{1}^{2}$ in gleichen Abständen vom Puncte $\boldsymbol{P}^{0}$ begrenst wird, so da/s $a_{1} \boldsymbol{P}^{\prime \prime}=b_{1} \boldsymbol{P}^{\prime \prime}$ ist. Ferner geht die Curve $\boldsymbol{P}^{2}$ durch die Mittelpuncte der gegebenen Curven $C^{2}$ und $C_{1}^{2}$, so wie durch die Mitten der 6 Seiten des vollständigen Vierecks qrst, und durch die drei Schnittpuncte, etwa $\mathfrak{a}, \mathfrak{b}$ und $\mathfrak{c}$, der drei Paar Gegenseiten desselben; demsufolge hat die Curve $\boldsymbol{P}^{2}$ die drei Geraden, welche die Milten der Gegenseiten verbinden, zu Durchmessern, und deren gemeinsamen Schnittpunct zum Mittelpunct, so da/s also ihr Mittelpunct im Schwerpunct der 4 Puncte $q, r, s, t$ liegt. - Die Curve $\boldsymbol{S}_{2}^{3}$ berührt die Gerade $G_{\infty}$ in den nämlichen beiden Puncten $x$ und $x_{1}$, in welchen letztere von der Curve $\boldsymbol{P}^{2}$ geschnitten wird; ferner berührt sie insbesondere die zwei Paar Asymptoten der gegebenen Curven $\boldsymbol{C}^{2}$ und $\boldsymbol{C}_{1}^{2}$, und auch jene zweimal 4 Tangenten $\boldsymbol{S}_{2}^{\prime \prime}$ derselben, so wie ferner die 6 Seiten des vollständigen Vierecks $q \boldsymbol{r} s t$ und die durch die Ecken des Dreiecks abc den Gegenseiten desselben parallel gezogenen drei Geraden.

Die angegebenen Eigenschaften haben noch eine weitere Ausdehnung. Denkt man sich den durch $\boldsymbol{C}^{2}$ und $\boldsymbol{C}_{1}^{2}$ bestimmten Kegelschnitt-Büschel, $\boldsymbol{B}\left(\boldsymbol{C}^{2}\right)$, mit den 4 Grundpuncten $q, r, s$ und $t$, d. h. alle Kegelschnitte, welche mit den beiden gegebenen die nämlichen vier (reellen oder imaginären) Puncte $q, r, s, \ell$ gemein haben; so kann man sagen: „Die Curve $\boldsymbol{P}^{2}$ sei zugleich der Ort der Mittelpuncte aller dieser Kegelschnitte $\boldsymbol{B}\left(C^{2}\right)$, so da/s jeder $\boldsymbol{P}_{\text {ol }} \boldsymbol{P}$ allemal zugleich der Mittelpunct irgend eines derselben ist, und auch umgekehrt." Und: „Die Curve $\mathbf{S}_{2}^{3}$ sei zugleich der Ort der Asymptoten aller dieser Kegelschnitte $B\left(C^{2}\right)$, so da/s jede Doppelsehne $\boldsymbol{S}_{2}$ zugleich eine Asymptote irgend eines derselben ist, und auch umgekehrt." Und zwar ist dabei der jedesmalige Pol $\boldsymbol{P}$ nicht allein die Mitte der Sehnen $a b, a_{1} b_{1}$ der beiden gegebenen Kegelschnitte $C^{2}$ und $C_{1}^{2}$, sondern er ist die gemeinsıme Mitte aller Sehnen, welche die zugehörige $\boldsymbol{S}_{2}$ mit sämmtlichen Kegelschnitten $\boldsymbol{B}\left(C^{2}\right)$ bildet, und welche in stetiger Folge alle Grö/sen, 
von 0 bis $\infty$, enthalten. Nämlich unter den Kegelschnitten giebt es jedesmal einen, etwa $C_{2}^{2}$, welcher die $\boldsymbol{S}_{2}$ in $\boldsymbol{P}$ berührt, dessen Sehne $a_{2} b_{2}$ somit $=0$ ist; ferner einen andern, etwa $C_{n}^{2}$, welcher $\boldsymbol{S}_{2}$ zur Asymptote hat, dessen Sehne somit $=\infty$ wird; und dazwischen liegen alle andern (reellen) Sehnen. Der Mittelpunct des letztern Kegelschnitts $\boldsymbol{C}_{n}^{2}$ ist derjenige Pol $\boldsymbol{P}_{n}(=\boldsymbol{P})$, in welchem $\boldsymbol{S}_{2}$ die Curve $\boldsymbol{P}^{2}$ zum zweiten Mal schneidet. Werden in allen Kegelschnitten, $\boldsymbol{B}\left(\boldsymbol{C}^{2}\right)$, nach irgend einer Richtung parallele Durchmesser $\alpha, \alpha_{1}, \alpha_{2}, \ldots$ gezogen, so treffen sich die ihnen conjugirten Durchmesser $\beta, \beta_{1}, \beta_{2}, \ldots$ sämmtlich in irgend einem Pol $\boldsymbol{P}$, und auch umgekehrt. Bei demjenigen Kegelschnitte, etwa $\boldsymbol{C}_{m}^{2}$, welcher den jedesmaligen Pol $\boldsymbol{P}$ zum Mittelpunct hat, fällt der Durchmesser $\alpha_{m}$ auf $\boldsymbol{S}_{2}$ und dessen conjugirter $\boldsymbol{\beta}_{n}$ auf die Tangente der Curve $\boldsymbol{P}^{2}$ im Pol $\boldsymbol{P}$. Also: Diejenigen Durchmesser $\alpha_{m}$ in allen Kegelschnitten $\boldsymbol{B}\left(\boldsymbol{C}^{2}\right)$, deren conjugirte $\beta_{m}$ die Curve $\boldsymbol{P}^{2}$ berühren, sind zugleich die gesammten Asymptoten derselben Kegelschnitte und umhüllen die Curve $\boldsymbol{S}_{2}^{3}$; und eben so: Die Tangenten $\left(\boldsymbol{S}_{2}^{\prime \prime}\right)$ aller Kegelschnitte $\boldsymbol{B}\left(C^{2}\right)$, in denjenigen Puncten, in welchen sie von ihrer Mittelpuncts-Curve $\boldsymbol{P}^{2}$ geschnitten werden, sind die nämlichen Asymptoten und umhüllen dieselbe Curve. - Unter $\boldsymbol{B}\left(\boldsymbol{C}^{2}\right)$ befinden sich, im Allgemeinen, zwei Parabeln; dieselben berühren die Curve $\mathcal{S}_{2}^{3}$ in den vorgenannten Puncten $x$ und $x_{1}$, und ihre Axen sind den Asymptoten der Curve $\boldsymbol{P}^{2}$ parallel. - Die Asymptoten jedes Kegelschnitts des $\boldsymbol{B}\left(C^{2}\right)$ sind irgend einem Paar conjugirten Durchmessern der Curve $\boldsymbol{P}^{2}$ parallel, und auch umgekehrt. U. s. w.

Da die drei Paar Gegenseiten des Vierecks qrst, als specielle Kegelschnitte, mit zum $\boldsymbol{B}\left(\boldsymbol{C}^{2}\right)$ gehören, so finden die angegebenen Eigenschaften, mit einiger Modification, auch für dieselben allein Anwendung, wodurch man mehrere, theils bekannte Sätze über das vollständige Viereck erhält.

2. ,Im Falle (B.) ist der Ort des Pols $P$ eine Curve $8^{\text {ten }}$ Grads, $\boldsymbol{P}^{8}$, und 22 ${ }^{\text {ster }}$ Classe, und der Ort der Doppelsehne $\boldsymbol{S}_{2}$ ist eine Curve

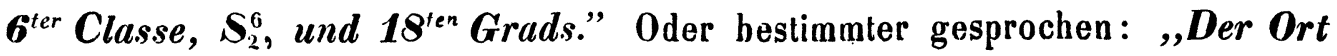
derjenigen Transversale $\boldsymbol{S}_{2}$, welche in zwei gegebenen Kegelschnitten $\boldsymbol{C}^{2}$ und $C_{1}^{2}$ gleiche Sehnen, $a b=a_{1} b_{1}$, bildet, ist eine Curve $6^{\text {ter }}$ Classe und $18^{\text {ten }}$ Grads, und der Ort des gemeinschaftlichen Schwerpuncts $P$ beider Sehnen ist eine Curve $8^{\text {ten }}$ Grads und 22 ${ }^{\text {ster }}$ Classe." Von beiden Curven sind unter andern folgende nähere Eigenschaften anzugeben.

Crelle's Jonrnal f. d. M. Bd. XLVII. Heft 1. 
Die Curve $\boldsymbol{P}^{8}$ hat die 4 Schnitte $q, r, s, t$ von $C^{2}$ und $C_{1}^{2}$ zu dreifachen Puncten; zudem hat sie noch 5 bestimmte Puncte $p$ zu Doppelpuncten, welche zugleich in der vorigen Curve $\boldsymbol{P}^{2}$ (1.) liegen, so dafs also diese 5 Puncle und jene 4 Schnitte zusammen die obigen 9 Pole $\boldsymbol{P}_{3}$ (\$. 17.) vertreten. Die Curve $\boldsymbol{P}^{8}$ geht auch durch die Mitten der 6 Seiten des vollständigen Vierecks qrst, schneidet sich also daselbst mit der Curve $\boldsymbol{P}^{2}$, was mit jenen $5 p$ zusammen die volle Zahl gemeinschaftlichen Puncte beider Curven ausmacht. Ferner geht die Curve $\boldsymbol{P}^{8}$ durch die Mitten der 4 gemeinschaftlichen Tangenten der gegebenen Curven $C^{2}$ und $C_{1}^{2}$, und berührt diese in ihren im Unendlichen liegenden Puncten $\alpha$ und $\beta, \alpha_{1}$ und $\beta_{1}$, so dafs sie mit denselben die zwei Paar Asymptoten gemein hat. Ihre übrigen 4 gemeinschaftlichen Puncte mit der Geraden $\boldsymbol{G}_{\infty}$ bestehen aus zwei Paaren, $\boldsymbol{y}$ und $\boldsymbol{y}_{1}$, $z$ und $z_{1}$, welche durch jene zwei Paare $\alpha$ und $\beta, \alpha_{1}$ und $\beta_{1}$, eben so bestimmt werden, wie oben, nämlich dafs

$$
\alpha y \alpha_{1} y_{1} \text { und } \beta y \beta_{1} y_{1} ; \alpha z \beta_{1} z_{1} \text { und } \alpha_{1} z \beta z_{1}
$$

harmonisch sind. Und gleicherweise werden die Richtungen der zugehörigen Asymptoten durch die obige Construction (\$.17.) gefunden.

Die Curve $\boldsymbol{S}_{2}^{6}$ hat die Gerade $\boldsymbol{G}_{\infty}$ zur vierfachen Tangente und berührt sie in den nämlichen 4 Puncten $y, y_{1}, z$ und $z_{1}$, in welchen dieselbe von der Curve $\boldsymbol{P}^{8}$ geschnillen wird. Die Curve $\boldsymbol{S}_{2}^{6}$ berührt insbesondere auch die 6 Seiten des vollständigen Vierecks grst, so wie die vier gemeinschaftlichen Tangenten der gegebenen Curven $C^{2}$ und $C_{1}^{2}$; und diese Curven selbst berührt sie in deren unendlich entfernten Puncten $\alpha$ und $\beta, \alpha_{1}$ und $\beta_{1}$ vierpunctig, hat somit deren Asymptoten mit ihnen gemein, und zwar ist jede dieser Asymptoten für 4 gemeinschaftliche Tangenten der betreffenden Curven zu zählen, so dafs also alle 12 Tangenten angegeben sind, welche $\boldsymbol{S}_{2}^{6}$ mit $\boldsymbol{C}^{2}$ oder $C_{1}^{2}$ gemein hat. Von den 18 Puncten, welche die Curve $\boldsymbol{S}_{2}^{6}$ mit der Geraden $G_{\infty}$ gemein hat, sind bereits 12 angegeben, nämlich die 4 Berührungspuncte $y, y_{1}, z$ und $z_{1}$, doppelt gezählt, und die 4 Puncte $\alpha, \beta, \alpha_{1}$ und $\beta_{1}$; die noch fehlenden 6 Puncte werden ähnlicherweise bestimmt, wie oben die 18 Puncte $Q_{0}$ ( $\$$. 17.).

II. Besteht die Basis aus $4 C^{\mathfrak{1}}$, d. h. aus vier beliebigen Geraden, etwa $\boldsymbol{A}, \boldsymbol{B}, \boldsymbol{C}$ und $\boldsymbol{D}$, so bilden dieselben ein vollständiges Vierseit $\boldsymbol{A B C D}$, dessen 6 Ecken als Doppelpuncte der Basis anzusehen sind. Dabei treten noch gröfsere Änderungen ein, als vorhin, und zwar der Art: 
2. St einer, algeb. Curven, welche Mittelp. haben, u. Eigens. allgem. algeb. Curven. 59

,Dafs dabei die Curve $\boldsymbol{P}^{10}$ aus drei Kegelschnitten $\boldsymbol{P}^{2}$ und aus den gegebenen 4 Geraden selbst, und die Curve $\boldsymbol{S}_{2}^{9}$ aus drei verschiedenen Curven $\mathbf{S}_{2}^{3}$ besteht."

Nämlich es sind hier dreierlei Doppelsehnen zu unterscheiden. Werden die Schnittpuncte der Transversale $\boldsymbol{S}_{2}$ mit den Geraden $\boldsymbol{A}, \boldsymbol{B}, \boldsymbol{C}, \boldsymbol{D}$ beziehlich durch $a, b, c, d$ bezeichnet, so sind folgende drei Fälle möglich; entweder hahen:

a) Die Sehnen $a b$ und $c d$, oder

$\beta)$ Die Sehnen $a c$ und $b d$, oder

$\gamma)$ Die Sehnen ad und bc

die nämliche Mitte $\boldsymbol{P}$. Werden ferner die drei Paar Gegenecken des Vierseits $A B C D$, nämlich $A B$ und $C D, A C$ und $B D, A D$ und $B C$ beziehlich durch $e$ und $e_{1}, f$ und $f_{1}, g$ und $g_{1}$, so wie dessen Diagonalen $e e_{1}, f f_{1}, g g_{1}$ durch $E, F, G$ und deren Mitten durch $e_{0}, f_{0}, g_{0}$ und deren gegenseitigen Schnittpuncte $\boldsymbol{E} \boldsymbol{F}, \boldsymbol{E} \boldsymbol{G}, \boldsymbol{F G}$ durch $\mathfrak{g}, f, e$ bezeichnet: so lassen sich die drei Fälle auf die drei einfachen Vierecke beziehen, welche in dem vollständigen Vierseit $\boldsymbol{A B C D}$ enthalten sind, und dadurch folgendermafsen bestimmter unterscheiden.

a. Dem Falle $\left(\alpha_{0}\right)$ entspricht das Viereck $f g f_{1} g_{1}$, welches $\boldsymbol{A}$ und $\boldsymbol{B}$, $\boldsymbol{C}$ und $\boldsymbol{D}$ zu Gegenseiten und $\boldsymbol{F}, \boldsymbol{G}$ zu Diagonalen hat.

b. Dem Falle ( $\beta$.) entspricht das Viereck ege 1. $_{1}$, welches $\boldsymbol{A}$ und $\boldsymbol{C}$, $\boldsymbol{B}$ und $\boldsymbol{D}$ zu Gegenseiten und $\boldsymbol{E}, \boldsymbol{G}$ zu Diagonalen hat.

c. Dem Falle $\left(\gamma\right.$ ) entspricht das Viereck $e f e_{1} f_{1}$, welches $\boldsymbol{A}$ und $\boldsymbol{D}$, $\boldsymbol{B}$ und $\boldsymbol{C}$ zu Gegenseiten und $\boldsymbol{E}, \boldsymbol{F}$ zu Diagonalen hat.

Nach dieser Beziehung wird, wie man sieht, die Doppelsehne $\boldsymbol{S}_{2}$ durch die zwei einfachen Sehnen zwischen den beiden Paar Gegenseiten des betreffenden Vierecks bestimmt, und demgemäfs zerfallen die beiden Ortscurven in die genannten Theile, und zwar wie folgt.

In Rücksicht jedes der drei einfachen Vierecke fyf $f_{1}$, ege $g_{1}$ und efe $f_{1} f_{1}$, für sich betrachtet, ist der Ort des Pols $\boldsymbol{P}$ die nümliche Curve $\boldsymbol{P}^{2}$, in welcher die Mittelpuncte des dem Viereck umgeschriebenen Kegelschnitt-Büschels $\boldsymbol{B}\left(C^{2}\right)$ liegen, und der Ort der zugehörigen Doppelsehne $\boldsymbol{S}_{2}$ ist die nümliche Curve $\mathbf{S}_{2}^{3}$, welche von den gesammten Asymptoten derselben Kegelschnitte umhüllt wird. Jede der drei Curven $\mathbf{S}_{2}^{3}$ hat die Gerade $G_{\infty}$ zur Doppeltangente und berührt sie in den nümlichen Puncten, in welchen dieselbe von der zugehörigen Curve $\boldsymbol{P}^{2}$ geschnitten 
60 2. St einer, algeb. Curven, welche Mittelp. haben, u. Eigens. allgem. alyeb. Curven.

wird. Überhaupt verhalten sich die jedesmaligen beiden Curven $\boldsymbol{P}^{2}$ und $\boldsymbol{S}_{2}^{3}$ zu dem zugehörigen Viereck gerade eben so, wie vorhin (I. 1.) die gleichbenannten Curven zu dem Viereck qrst. Nur ein Umstand, betreffend das Verhalten der drei Curven $\boldsymbol{P}^{2}$ gegen einander, mag hier noch besonders hervorgehoben werden. $\mathrm{Zu}$ diesem $\mathrm{Zweck}$ unterscheide man die $3 \boldsymbol{P}^{2}$ nach den Schnittpuncten der Diagonalen der respectiven Vierecke durcb $\boldsymbol{P}_{\mathfrak{s}}^{2}, \boldsymbol{P}_{\mathrm{f}}^{2}$ und $\boldsymbol{P}_{\mathrm{g}}^{2}$. Alsdann findet (aufserdem, dafs jede dieser Curven durch die Mitten der 4 Seiten und durch den Schnitt der Diagonalen des zugehörigen Vierecks geht) Folgendes statt:

$\boldsymbol{u}^{0}$. Die Curve $\boldsymbol{P}_{c}^{2}$ geht durch die Mitten $f_{1}, g_{0}$ der Diagonalen des Vierecks $f g f_{1} g_{1}$, so wie durch die Schnitte $e, e_{1}$ seiner zwei Paar Gegenseiten, welche zugleich ein Paar Gegenecken des vollständigen Vierseits $\boldsymbol{A B C D}$ sind; ihr Mittelpunct liegt in der Mitte der Geraden $f_{0} g_{0}$ und ist der Schwerpunct der vier Ecken $f, g, f_{1}, g_{1}$.

$b^{\circ}$. Die Curve $\boldsymbol{P}_{f}^{2}$ geht durch die Mitten $e_{0}, g_{0}$ der Diagonalen des Vierecks ege $_{1} g_{1}$ und durch die Schnitte $f, f_{1}$ seiner Gegenseiten; ihr Mittelpunct liegt in der Mitte der Geraden $e_{0}, g_{0}$, etc.

$c^{\circ}$. Die Curve $\boldsymbol{P}_{\mathrm{g}}^{2}$ geht durch die Mitten $\boldsymbol{e}_{0}, f_{0}$ der Diagonalen und durch die Schnitte $g, g_{1}$ der Gegenseiten des Vierecks $e e_{1} f_{1}$; ihr Mittelpunct liegt in der Mitte der Geraden $e_{0} f_{0}$.

Demnach gehen die drei Curven $\boldsymbol{P}_{\mathfrak{e}}^{2}, \boldsymbol{P}_{\mathrm{f}}^{2}, \boldsymbol{P}_{\mathfrak{g}}^{2}$ zusammen durch alle 6 Ecken des gegebenen vollständigen Vierseits $\boldsymbol{A B C D}$, jede durch ein Paar Gegenecken $e$ und $e_{1}, f$ und $f_{1}, g$ und $g_{1}$; zudem schneiden sie einander paarweise in den Mitten $g_{0}, f_{0}, e_{0}$ der drei Diagonalen desselben und haben die Abstände dieser drei Mitten von einander zu Durchmessern $\left(g_{10} f_{0}, g_{0} e_{0}, f_{0} e_{0}\right)$; und da nun diese drei Mitten bekanntlich in einer Geraden liegen: so liegen also auch die Mittelpuncte der drei Curven in derselben Geraden. - Durch die drei Puncte, etwa $3 p$, in welchen sich irgend zwei der drei Curven aufserdem noch schneiden, mu/s nothwendig auch die dritte Curve gehen; und die Puncte haben die besondere Eigenschaft, da/s jedem drei Doppelsehnen $\mathbb{S}_{2}$ zugehören. Diese 3 Puncte $p$ und die 6 Ecken efge $f_{1} g_{1}$ des gegebenen Vierseits $\boldsymbol{A B C D}$ vertreten zusammen die obigen 9 Pole $\boldsymbol{P}_{3}$ (\$. 17.). Unter den 11 Räumen, in welche die Ebene durch die vier Geraden $A, B, C, D$ getheilt wird, befinden sich, im Allgemeinen, drei ganz begrenzte, nämlich zwei Dreiecke und ein Viereck: in jedem dieser drei Räume liegt einer der drei Pole p. Dieselben vier Geraden, zu je drei genommen, bilden vier 
2. St einer, algeb. Curven, welche Mittelp. haben, u. Eigens. allgem. algeb. Curven. 61

Dreiecke: Die Ecken und der Schwerpunct jedes dieser Dreiecke liegen mit den drei Polen $p$ zusummen iı irgend einem Kegelschnitte. Jeder dieser vier Kegelschnitte ist nebstdem dadurch bestimmt: da/s seine Tangenten in den Ecken des Dreiecks durch die Mitten derjenigen Strecken gehen, welche von den die Ecken bildenden Geraden (Seiten) auf der jedesmaligen vierten Geraden begrenzt-werden; z. B. bei dem durch $\boldsymbol{B}, \boldsymbol{C}, \boldsymbol{D}$ gebildeten Dreieck $e_{1} f_{1} g_{1}$ gehen die Tangenten des zugehörigen Kegelschnitts in den Ecken $e_{1}, f_{1}, g_{1}$ beziehlich durch die Mitten der Strecken $f g$, eg, ef auf der Geraden $\boldsymbol{A}$.

Da für jeden der oben betrachteten Pole $\boldsymbol{P}$ die innere Polare $\boldsymbol{J}^{3}$ in Bezug auf das jedesmalige einfache Viereck aus $\boldsymbol{J}^{2}+\boldsymbol{S}_{2}$ besteht, so kann man sagen: Soll ein Punct in der Ebene eines gegebenen einfachen Vierecks die Eigenschaft haben, da/s die 8 Endpuncte der durch ihn swischen je zwei aufeinander folgende Seiten des Vierecks gezogenen vier Sehnen $\mathbf{S}$ in irgend einem Kegelschnitte $J^{2}$ liegen, so mu/s er ein Pol $\boldsymbol{P}$ sein, oder, so ist sein Ort die nämliche obige Curve $\boldsymbol{P}^{2}$. Und werden durch denselben Punct ferner zwischen jeder Seite und jeder der beiden Diagonalen gleicherweise die durch den Punct gehülfteten Sehnen $\mathbf{S}$ gezogen, was 8 neue Sehnen giebt, so liegen die 24 Endpuncte aller 12 einfachen Sehnen $\mathbf{S}$ in irgend einer Curve $4^{\text {ten }}$ Grads $\boldsymbol{J}^{4}$, welche $\boldsymbol{P}$ zum Mittelpunct hat.

\section{\$. 19.}

In wiefern das Zerfallen der innern Polaren auch bei den Basen höhern Grads statt findet, welche Umstände dabei obwalten, und welche Eigenschaften und Sätze sich daraus ergeben, habe ich nicht untersucht; nur über die Basis $5^{\text {ten }}$ Grads habe ich einen flüchtigen Versuch gemacht und nebstdem den Ort der Doppélsehnen für jede Basis bestimmt, wobei sich unter andern folgende Resultate herausstellten.

Ist die Basis eine allgemeine Curve $5^{\text {ten }} \mathrm{Grads}, C^{5}$, so kann die innere Polare $\boldsymbol{J}^{4}$ irgend eines Pols $\boldsymbol{P}$ möglicherweise entweder
1) aus $\boldsymbol{J}^{3}+\boldsymbol{S}_{2}$,
2) aus $\boldsymbol{J}^{2}+2 S_{2}$
3) aus $4 S_{2}$
oder 4) aus $\boldsymbol{J}^{2}+\boldsymbol{J}_{1}^{2}$

bestehen. Nun zeigt sich zunächst, dafs hierbei der Ort des Pols $\boldsymbol{P}$ nicht gleicherweise eine Curve sein kann, wie bei den vorhergehenden Beispielen, sondern dafs vielmehr den drei ersten Fällen nur eine bestimmte Anzahl Pole 
entsprechen und dafs namentlich der Fall (3.) nur unter besondern Umständen vorkommt.

Soll z. B. der Fall (1.) eintreten, so mufs nothwendigerweise der Pol $\boldsymbol{P}$ in der Basis $C^{5}$ selbst liegen, und zwar mufs er einerseits nicht nur die Mitte, sondern zugleich der fünfte Schnitt der Doppelsehne $\boldsymbol{S}_{2}$ mit der Basis, und andererseits der Miltelpunct der durch ihn gehenden Curve $J^{3}$ sein, wobei sodann in Rücksicht derjenigen unter den zugehörigen 10 Sehnen $S$, weeche auf die Tangente der Basis fällt und deren Endpuncte im Berührungspuncte, in $\boldsymbol{P}$, vereint liegen, der eine dieser Endpuncte als in $\boldsymbol{S}_{2}$ und der andere als in $\boldsymbol{J}^{3}$ liegend anzusehen ist, so dafs also die Curve $\boldsymbol{J}^{3}$ aufserdem noch durch die 14 Endpuncte von 7 andern Sehnen $\boldsymbol{S}$ geht, und die beiden übrigen Sehnen in $\boldsymbol{S}_{2}$ liegen. Da nun offenbar nicht jeder Punct in der Basis diese Eigenschaft haben kann, so ist klar, dafs der genannte Fall nur für einzelne bestimmte Pole eintreten wird. Die Anzahl dieser Pole wird durch folgenden Satz bedingt.

,Der Ort aller Doppelsehnen $\boldsymbol{S}_{2}$, welche in der gegebenen Basis $\boldsymbol{C}^{5}$ überhaupt möglich sind, ist eine Curve $45^{\text {ster }}$ Classe, $\boldsymbol{S}_{2}^{45}$, und der Ort ihrer Mitten, $\boldsymbol{P}$, ist höchstens eine Curve $45^{\text {sten }}$ Grads, $\boldsymbol{P}^{45}$."

Demnach können also dem Falle (1.) nur solche Pole genügen, welche gemeinschaftliche Puncte der beiden Curven $\boldsymbol{C}^{5}$ und $\boldsymbol{P}^{45}$ sind, und somit kann es höchstens $5.45=225$ solche Pole geben. Darin sind nun aber auch die beiden Fälle (2.) und (3.) mit inbegriffen, wie leicht zu sehen, und es bleibt zu entscheiden, in wiefern dieselben möglich sind, oder nicht; denn der Fall (2.) erfordert, dafs der Pol $\boldsymbol{P}$ zugleich ein Doppelpunct der Curve $\boldsymbol{P}^{45}$ sein mufs, und der Fall (3.) dagegen erheischt, dafs der Pol ein solcher Punct der Basis $C^{5}$ sein mufs, in welchem dieselbe von der zugehörigen Tangente vierpunctig berührt wird, oder dafs er ein Doppelpunct derselben sein mufs; so dafs also dieser Fall, im Allgemeinen, gar nicht vorkommt.

Hieraus ergiebt sich durch Umkehrung und Projection der folgende Satz: ,Zieht man durcle einen Wendepunct Y einer beliebigen gegebenen Curve $3^{\text {ten }}$ Grads $\mathfrak{J}^{3}$ irgend 7 Secanten $\mathfrak{S}$, welche dieselbe in 14 neuen Puncten schneiden, so geht jede durch diese 14 Puncte gelegte Curve $5^{\text {ten }}$ Grads $\mathbb{6}^{5}$ nothwendig auch durch jenen Wendepunct; oder jede $\mathfrak{S}^{5}$, welche durch irgend 14 der genannten 15 Puncte geht, geht allemal auch durch den fünfzehnten." Auch giebt es dabei in jeder Curve $\mathfrak{C}^{5}$ eine solche durch $\mathfrak{P}$ gehende Secante $\mathfrak{S}_{2}$, von welcher sie in zwei Paar Puncten 
¿ und $a_{1}, b$ und $b_{1}$ geschnitten wird, die beide zu $\mathfrak{Y}$ und dem Schnitte, etwa $\mathfrak{D}$, der $\mathfrak{S}_{2}$ mit der Harmonischen $H(\$ .11$.$) von \mathfrak{S}$ in Bezug auf die Curve $\mathfrak{S}^{3}$, zuyeordnet harmonisch sind, d. h. souvohl a $\mathfrak{S} a_{1} \mathfrak{D}$ als $b \mathfrak{S} b_{1} \mathfrak{Z}$ sind harmonisch.

Dafs weiter auch der Fall (4.) bei einer allgemeinen Basis $C^{5}$ vorkommen kann, geht umgekehrt daraus hervor: dafs man durch die 10 Endpuncte von 5 beliebigen Durchmessern eines gegebenen Kegelschnitts $\boldsymbol{J}^{2}$ immerhin eine solche Curve legen kann, ohne dafs dieselbe etwas von ihrer Allgemeinheit einbüfst; aber alsdann mufs deren innere Polare, welche dem Mitlelpuncte $\boldsymbol{P}$ von $\boldsymbol{J}^{2}$ entspricht, nothwendig aus diesem Kegelschnitte und aus irgend einem andern mit ihm concentrischen Kegelschnitte $\boldsymbol{J}_{1}^{2}$ bestehen. Es wàre daher zu untersuchen: „Ob der Fall (4.) auch nur für einzelne bestimmte Pole eintrete, oder ob für ihn der Ort des Pols $\boldsymbol{P}$ irgend eine Curve sei, und welche? - Bei diesem Falle kann sich möglicherweise auch das ereignen, dafs für irgend einen Pol fünf Doppelsehnen $\boldsymbol{S}_{2}$ entstehen, deren Endpuncte jedoch immerhin in $\boldsymbol{J}^{2}+\boldsymbol{J}_{1}^{2}$ liegen; ein solcher Pol würde alsdann ein fünffacher Punct der Curve $\boldsymbol{P}^{45}$ sein.

Übrigens läfst sich der Ort der Doppelsehnen allgemein für jede beliebige Basis angeben, nämlich:

„Der Ort aller Doppelsehnen $\boldsymbol{S}_{2}$, welche in einer gegebenen allgemeinen Curve $m^{\text {ten }}$ Grads überhaupt möglich sind, ist eine Curve von $\operatorname{der}{ }_{8}^{3} m(m-1)(m-2)(m-3)^{\text {ten }}$ Classe.

\section{S. 20.}

Aufser der gleich Anfangs namhaft gemachten Eigenschaft der beiden Polaren $\boldsymbol{A}^{m-1}$ und $\boldsymbol{J}^{m-1}$ jedes Pols $\boldsymbol{P}$ in Bezug auf eine gegebene Basis $\boldsymbol{C}^{m}$ (\$. 13. II.), wären nun noch andere gegenseitige Beziehungen beider Polaren, so wie auch das Verhalten der innern Polare $J^{m-1}$ zu andern, mit ihr in Verbindung stehenden Curven, zu erforschen. Ich habe darüber ebenfalls nur einen schwachen Versuch gemacht, der indessen doch schon einige nicht ganz uninteressante Resultate geliefert hat; wie aus dem Folgenden erhellen wird. Eine vollständigere Behandlung dieses Gegenstandes überlasse ich Andern.

I. Ist die gegebene Basis eine Curve $3^{\text {ten }} \mathrm{Grads}, C^{3}$, so gehen durch jeden beliebigen Pol $P$ drei Sehnen $S$, oder $a \dot{a}_{1}, b b_{1}$ und $c c_{1}$, deren 6 Endpuncte, die fortan alle durch $a$ bezeichnet werden sollen, also deren 6 Endpuncte $a$ in der Polare $\boldsymbol{J}^{2}$ liegen. Die Basis wird von jeder der drei Sehnen noch in 
64 2. Steiner, algeb. Curven, welche Mittelp. haben, u. Eigens. allgem. algeb. Curven.

irgend einem dritten Puncte geschnitten, bezieblich $\alpha, \beta$ und $\gamma$, oder wenn jeder dieser Schnitte durch $\alpha$ bezeichnet wird, so wird sie von den $3 \boldsymbol{S}$ noch in 3 Puncten $\alpha$ geschnitten.

„Die 3 Puncte a liegen allemal in irgend einer Geraden, elwa R.” Und: „Diese Gerade $\boldsymbol{R}$ ist jedesmal eine (reelle oder ideelle) gemeinschaftliche Secante der beiden Polaren $A^{2}$ und $J^{2}$ des zugehörigen Pols $P$ in Bezug auf die Basis $C^{3}$, so da/s also die Geruden $G_{\infty}$ und $\boldsymbol{R}$ immer ein Paur sich entgegenstehende gemeinschattiche Secanten der beiden Polaren sind. Ferner: „Die Gerade $R$ ist stets der sweiten äu/sern Polare $A^{\prime}$ desselben Pols in Bezug auf die Basis parallel." Liegt der Pol $P$ insbesondere in der Basis sclbst, so fallen $R$ und $\boldsymbol{A}^{\mathbf{1}}$ auf die zugehörige Tangente.

Soll die Gerade $R$ durch irgend einen in der $B$ asis $C^{3}$ yegebenen Punct a gehen, so ist der Ort des ihr entsprechenden Pols $P$ eine Curve $3^{\text {ten }}$ Grads, etwa $\boldsymbol{P}_{\alpha}^{3}$, welche in a einen Doppelpunct und mil der Basis deren im Unendlichen liegende drei Puncte $a_{\infty}$ gemein und folylich mit derselben parallele Asymploten hat. Und soll die Gerade $R$ durch swei in der Basis gegebene Puncte $\alpha$ und $\beta$ gehen, wodurch sie und auch ihr dritter Schnitt $\gamma$ mit der Busis bestimmt ist, so entsprechen ihr noch 6 verschiedene Pole $P$, in welchen nämlich die den Puncten $\alpha, \beta$ und $\gamma$ entsprechenden Ortscurven $\boldsymbol{P}_{\alpha}^{3}, \boldsymbol{P}_{\beta}^{3}$ und $\boldsymbol{P}_{\gamma}^{3}$, au/ser in jenen drei Puncten $a_{\infty}$, einander schneiden, und welche somit in irgend einem Kegelschnitte liegen (weil die $3 u_{\infty}$ sich in $G_{\infty}$ befinden); und zuar ist dieser Kegelschnilt zugleich die erste äu/sere Polare, etwa $A_{r}^{2}$, des nach der Richtung der Geraden $R$ im Unendlichen liegenden Punctes $r_{\infty}$ in Bezug auf die Basis. Die zweiten Polaren, $\boldsymbol{A}^{1}$, der 6 Pole $\boldsymbol{P}$ sind alle der Geraden $\boldsymbol{R}$ parallel. Wird die Gerade $R$ sich selbst parallel bewegt, so ändern sich zurar die drei Ortscurven $\boldsymbol{P}_{u}^{3}, \boldsymbol{P}_{\beta}^{3}$ und $\boldsymbol{P}_{\gamma}^{3}$ und mit ihnen sugleich auch ihre 6 Schnitte oder Pole P: aber der Kegelschnitt $A_{r}^{2}$, in welchem die letztern liegen, bleibt unveränderlich fest.

Soll die Polare $J^{2}$ durch einen in der Basis gegebenen Punct a gehen, so ist der Ort des zugehörigen Pols $\boldsymbol{P}$ eben so irgend eine Curve $3^{\text {ten }}$ Grads, $\boldsymbol{P}_{a}^{3}$, welche die $\boldsymbol{B}$ asis in $\boldsymbol{P}$ berührt, ihr ähnlich und mit ihr ähnlichliegend ist, also mit ihr parallele Asymptolen oder die drei Puncte $\|_{\infty}$ gemein hat. Und soll $J^{2}$ durch zwei in der Basis gegebene Puncte a und $b$ gehen, so entsprechen ihr noch 6 verschiedene Pole $P$, in welchen die Ortscurven $\boldsymbol{P}_{a}^{3}$ und $\boldsymbol{P}_{b}^{3}$, au/ser in den $3 a_{\infty}$, einander schneiden, und 
2. Steiner, algeb. Curven, welche Mittelp. haben, u. Eigens. allgem. alyeb. Curven. 65

welche somit in irgend einem Kegelschnitte liegen; und numentlich ist die Mitte der Geraden ab einer dieser 6 Pole. Daraus schliefst man den folgenden speciellen Satz:

„Über einer gegebenen Grundlinie ab, deven Endpuncte in einer gegebenen Curve $3^{\text {ten }}$ Grads $C^{3}$ liegen, lassen sich dieser Curve, im Allgemeinen, fünf verschiedene Parallelogramme einschreiben; und dabei liegen die füf Puncte, in denen die Diagonalen der einselnen Parallelogramme sich kreuzen, mit der Mitte der geineinsamen Grundlinie zusammen in irgend einem Kegelschnitte." Oder anders ausgesprochen: „Zu jeder Sehne ab in einer gegebenen Curve $3^{\text {ten }}$ Grads giebt es, im Allgemeinen, funf andere Sehnen die ihr gleich und parallel sind. Die Mitten solcher sechs Sehnen liegen allemal in irgend einem Kegelschnitt." Dieser Satz umfafst, wofern man die Sehne $a b$ unendlich klein werden oder in eine Tangente übergehen läfst, auch den bekannten Satz: „Da/s die Tangenten einer Curve $C^{3}$ zu 6 und 6 parallel sind, und da/s die Berührungspuncte von je 6 solchen Tungenten in einem Kegelschnitte liegen, nümlich in der ersten Polare $A_{0}^{2}$ des nach der Richtung der Tangenten im Unendlichen liegenden Punctes." - Hierbei bleiben noch viele Fragen zu erledigen, wie z. B. folgende. $Z u$ jeder Sehne ab gehören 6 Pole $P$, und diesen entsprechen 6 Gerade $R$ : welche Eigenschaft haben diese $6 \boldsymbol{R}$ ? Der durch die $6 \boldsymbol{P}$ gehende Kegelschnitt heifse $\boldsymbol{P}^{2}$, und der durch die Mitte von $a b$ und durch die Mitten der mit ihr zusammengehörigen 5 Sehnen gehende Kegelschnitt heifse $\boldsymbol{A}_{1}^{2}$; diese zwei Kegelschnitte berühren sich in der Mitte von $a b$, sind ähnlich und ähnlichliegend, und ibre entsprechenden Dimensionen verhalten sich, wie $1: 2$. Wird nun die Sehne $a b$ sich selbst parallel bewegt, so entsteht eine Schaar Kegelschnnitte $\boldsymbol{P}^{2}, \boldsymbol{S}\left(\boldsymbol{P}^{2}\right)$, und eben so eine $\boldsymbol{S}\left(\boldsymbol{A}_{1}^{2}\right)$ : welche Eigenschaft haben diese Kegelschnittschaaren? Und wenn man der Sehne $a b$ nacheinander alle Richtungen giebt: welche Beziehung haben dann alle $\boldsymbol{S}\left(\boldsymbol{P}^{2}\right)$ oder alle $\boldsymbol{S}\left(\boldsymbol{A}_{1}^{2}\right)$ zueinander? Unter jeder $\boldsymbol{S}\left(\boldsymbol{A}_{1}^{2}\right)$ befindet sich die vorgenannte Polare $\boldsymbol{A}_{0}^{2}$, und alle $\boldsymbol{A}_{0}^{2}$ bilden einen Büschel, $\boldsymbol{B}\left(\boldsymbol{A}_{0}^{2}\right)$. U. s. w.

II. Ist die Basis vom $4^{\text {ten }} \mathrm{Grad}, \boldsymbol{C}^{4}$, so gehen durch jeden Pol $\boldsymbol{P}$, im Allgemeinen, 6 Sehnen $S$, deren 12 Endpuncte $a$ in der Polare $\boldsymbol{J}^{3}$. liegen, die durch den Pol geht und ihn zum Mittel- und Wendepunct hat. Jede Sehne $\boldsymbol{S}$ schneidet die Basis, aufser in den $2 a$, in noch zwei andern Puncten $\alpha$, so dafs im Ganzen 12 Puncle $\alpha$ vorhanden sind. Auch die Curve $J^{3}$ wird von

Crelle's Journal f. d. M. Bd. XLVII. Heft 1. 
66 2. Steiner, algeb. Curven, welche Mittelp. haben, u. Eigens. allyem. algeb. Curven.

jeder Sehne $\boldsymbol{S}$ noch in einem dritten Puncte, etwa $p$, geschnitten, aber alle 6 Puncte $p$ liegen im Pol $\boldsymbol{P}$.

„Die 12 Puncle a liegen allemal in irgend einer Curve $3^{\text {ten }}$ Grads, etwa $\boldsymbol{R}^{3}$, welche auch durch den Pol $\boldsymbol{P}$ geht, ihn zum Wendepunct und zwar in demselben mit der Polare $J^{3}$ die Wendetangente, $\mathfrak{B}$, gemein hat, so da/s also beide Curven einander daselbst dreipunclig berühren, und da/s folglich ihre übrigen 6 gemeinschaftlichen Puncte, etwa $6 q$, in irgend einem Kegelschnitte $\boldsymbol{Q}^{2}$ liegen." - Die beiden Polaren $\boldsymbol{A}^{3}$ und $\boldsymbol{J}^{3}$ desselben Pols $\boldsymbol{P}$ haben mit der Geraden $\boldsymbol{G}_{\infty}$ die nämlichen drei Puncte, etwa $3 q_{\infty}$, gemein, und daher liegen ihre übrigen 6 gemeinschaftlichen Puncte, $q_{1}$, ebenfalls in irgend einem Kegelschnitte $\boldsymbol{Q}_{1}^{2}$ (\$.13. II.). Jene drei Puncte $\eta_{\infty}$ sind zugleich die Berührungspuncte der Curve $\boldsymbol{J}^{3}$ mit ihren drei Asymptoten, und die Gerade $\boldsymbol{G}_{\infty}$ ist die Harmonische ihres Wendepuncts $\boldsymbol{P}$ ( $($. 11.). In Rücksicht der Curve $\boldsymbol{R}^{3}$ bezeichne man die Harmonische ihres Wendepuncts $\boldsymbol{P}$ durch $\boldsymbol{H}$; dieselbe geht eben so durch die drei Berührungspuncte, etwa 3h, der aus $\boldsymbol{P}$ an $\boldsymbol{R}^{3}$ gelegten drei Tangenten (\$. 11.); , und durch diese 3 Puncte geht gleicherweise auch die Polare $A^{3}$, so da/s die übrigen 6 gemeinschaftichen Puncte, $q_{2}$, der beiden Curven $\boldsymbol{R}^{3}$ und $\boldsymbol{A}^{3}$ gleichfalls in irgend einem Kegelschnitte $Q_{2}^{2}$ liegen." Hieraus ist ersichtlich: „Da/s in projectivischer Hinsicht die beiden Curven $\mathrm{J}^{3}$ und $\boldsymbol{R}^{3}$ sich gegen die Polare $A^{3}$ (so wie auch gegen die Basis $C^{4}$ ) völlig gleich verhallen, so da/s sie ihre scheinbar verschiedene Rolle durch Projection (wobei $H$ ins Unendliche kommt) vertauschen, oder gänzlich verlieren und gegen $\boldsymbol{A}^{3}$ und $C^{4}$ eine völlig gleiche Stellung einnehmen können." [Hierbei entsteht die Frage: $O b$ nicht die von jedem Pol $P$ abhängigen drei Curven, nümlich die beiden Polaren $A^{3}, J^{3}$ und die Curve $\boldsymbol{R}^{3}$, alle durch dieselben 6 Puncte $q$ gehen, welche in einem Kegelschnitte $\boldsymbol{Q}^{2}$ liegen? Oder wenn dies nicht der Fall ist: Welche Beziehung alsdann die genannten 3 Kegelschnitte $Q^{2}, Q_{1}^{2}$ und $Q_{2}^{2}$ zu einander haben? und welche Beziehung ferner die zweite Polare $A^{2}$ des nämlichen Pols in Bezug auf die Basis (d. i. die erste Polare in Bezug auf $A^{3}$ ) zu denselben habe?] Es findet weiter Folgendes statt. „Die Harmonische $\boldsymbol{H}$ des Wendepuncts $\boldsymbol{P}$ der Curve $\boldsymbol{R}^{3}$ ist stets der dritten Polure $A^{1}$ desselben Punctes in Bezug auf die Basis parallel." Daher geht die dem Puncte $h_{\infty}$, der nach der Richtung von $H$ im Unendlichen liegt, entsprechende erste Polare $A_{h}^{3}$ in Bezug auf die Basis jedesmal durch den Pol P; und umgekehrt: für alle in dieser 
Polare $\boldsymbol{A}_{h}^{3}$ liegenden Pole $\boldsymbol{P}$ sind die ihnen, in Rücksicht der zugehörigen Curven $\boldsymbol{R}^{3}$ entsprechenden, Harmonischen $\boldsymbol{H}$ sämmtlich parallel, nümlich alle nach dem Puncte $h_{\infty}$ gerichtet; oder jedes System parallele Gerade in der Ebene sind als solche Harmonische $\boldsymbol{H}$ anzusehen, deren zugehörige Pole $P$ in derjenigen ersten Polare liegen, welche dem nach der Richtung der Geraden im Unendlichen gedachten Puncte entspricht. [Frage: Ist nicht die den Curven $J^{3}$ und $\boldsymbol{R}^{3}$ gemeinsame Wendetangente $\mathfrak{B}$ im Pol $\boldsymbol{P}$ mit den dem letztern entsprechenden vorgenannten Geraden $H$ und $A^{1}$ parallel? und wenn es so ist: wie verhalten sich dann die Abstände der drei Geraden $H, A^{1}$ und $\mathfrak{W}$ von einander? liegt etwa $\mathfrak{B}$ in der Mitte zwischen den beiden andern, so da/s alsdann die vier Geraden $\boldsymbol{H} \mathfrak{B} A^{1} G_{\infty}$ hormonisch sind?]

„Liegt der Pol $\boldsymbol{P}$ in der Basis $C^{4}$ selbst, so zerfällt $\boldsymbol{R}^{3}$ in $\boldsymbol{R}^{2}+\boldsymbol{R}$, und zwar ist die Gerade $R$ die Tangente der Basis im Pol $P$, somit zugleich die Wendetangente der Polare $J^{3}$ daselbst (\$. 14. I. 1.); und der Kegelschnitt $\boldsymbol{R}^{2}$ berührt die Basis in $\boldsymbol{P}$ dreipunctig." Nämlich von den obigen 12 Puncten $\alpha$ fallen hierbei 5 in $\boldsymbol{P}$, und von denselben kommen 2 auf die Berührung von $\boldsymbol{R}$ und $\boldsymbol{C}^{4}$, und die 3 andern auf die Berührung von $\boldsymbol{R}^{2}$ und $\boldsymbol{C}^{4}$. „Ist der Pol $\boldsymbol{P}$ insbesondere ein Wendepunct der Basis, so zerfält uuch noch der Kegelschnitt $\boldsymbol{R}^{2}$ in zwei Gerade, $\mathfrak{B}+\mathfrak{R}$, nümlich in die sugehörige Wendetangente $\mathfrak{W}$ der Basis und in irgend eine andere Gerade $\Re$, und da auch schon $R$ auf der Tangente liegt, so mu/s also in diesem Falle $\boldsymbol{R}^{3}$ aus der doppelten Wendetangente $\mathfrak{W}$ und aus einer (nicht durch $\boldsymbol{P}$ gehenden) Geraden $\Re$ bestehen." „Ist ferner der Pol insbesondere einer jener 32 Schnitte $\boldsymbol{P}^{0}$ der Basis und der obigen Curve $\boldsymbol{P}^{10}$ (\$. 17.), so besteht $\boldsymbol{R}^{3}$ wiederum aus der doppelten zugehörigen Tangente $\mathcal{S}_{2}^{0}(\$$. 17.) und aus irgend einer Geraden $\Re$." Nämlich von den 12 Puncten $\alpha$ fallen hier 6 in $\boldsymbol{P}$, zwei andere sind die äufsern Endpuncte von $\boldsymbol{S}_{2}^{0}$, und die vier übrigen liegen in der Geraden $\Re$. Bei diesen beiden besondern Fällen ist die Gerade $\Re$ der jedesmuligen Tangente $\mathfrak{W}$ oder $\boldsymbol{S}_{2}^{\prime \prime}$ der Basis, welche zugleich die $3^{\text {te }}$ Polare des Pols in Bezug auf die Basis ist, parallel."

„Liegt der Pol $\boldsymbol{P}$ in der Curve $\boldsymbol{P}^{10}$, uobei die Polare $\boldsymbol{J}^{3}$ aus $\boldsymbol{J}^{2}+\boldsymbol{S}_{2}$ besteht (\$. 17.), so zerfälll auch die Curve $R^{3}$ in $R^{2}+\mathbb{S}_{2}$, so da/s also in diesen Falle $J^{3}$ und $R^{3}$ die Doppelsehne $S_{2}$ zum gemeinsamen Bestandtheil haben; ferner ist dabei die Polure des Pols $\boldsymbol{P}$ in Bezug auf 
68 2. Steiner, algeb. Curven, welche Mittelp. haben, u. Eigens. allgem. algeb. Curven.

den Kegelschnitt $\boldsymbol{R}^{2}$, etwa $\boldsymbol{R}^{1}$, mit der dritten Polare $\boldsymbol{A}^{1}$ desselben Pols in Bezug auf die Basis parallel; und zwar sind beide, $R^{1}$ und $A^{1}$, mit der Doppelsehne $\boldsymbol{S}_{2}$ parallel."

III. Bei der Basis $C^{5}$ gehen durch jeden Pol $\boldsymbol{P}$ je 10 Sehnen $\boldsymbol{S}$, deren 20 Endpuncte $\boldsymbol{a}$ in der Polare $\boldsymbol{J}^{4}$ liegen, die den Pol zum Mittelpunct hat. Nun wird die Basis von den $10 S$ in noch $10.3=30$ andern Puncten $\alpha$ und die Polare $J^{4}$ wird von denselben in noch $10 \cdot 2=20$ neuen Puncten $a_{1}$ geschnitten, und zwar sind die letztern eben so paarweise Gegenpuncte in Rücksicht des Mittelpunctes $\boldsymbol{P}$, wie jene 20 Puncte $\boldsymbol{a}$.

„Durch die 30 Puncte a können unzählige Curven $\boldsymbol{6}^{\text {ten }}$ Grads, $\boldsymbol{R}^{6}$, gehen. Jede solche Curve schneidet die $10 \mathrm{~S}$, aufser in den $30 \alpha$, in $10 \cdot 3=30$ neuen Puncten $\alpha_{1}$.

„Solche 30 Puncte $\alpha_{1}$ liegen jedesmal mit jenen festen 20 Puncten $a_{1}$ susammen in irgend einer Curve $5^{\text {ten }}$ Grads, etwa $C_{1}^{5}$." Demnach gehen durch die 20 Puncte $a_{1}$ gerade eben soviele Curven $C_{1}^{5}$, als durch jene 30 Puncte $\alpha$ Curven $\boldsymbol{R}^{6}$ möglich sind; mittelst der 30 Puncte $\alpha_{1}$ bestimmen sie einander gegenseitig, so dafs jeder durch die $30 \alpha$ gehenden Curve $\boldsymbol{R}^{6}$ eine durch die $20 a_{1}$ gehende bestimmte Curve $C_{1}^{5}$ entspricht, und auch umgekehrt. Die Curve $R^{6}$ hat su der ihr entsprechenden Curve $C_{1}^{5}$ und zu der Basis $C^{5}$ völlig gleiche Beziehung. Die innern Polaren des Pols $P$ in Bezug auf die Curven $C^{5}$ und $C_{1}^{5}$ sind eine und dieselbe Curve $J^{4}$. Aus einem früheren Satze (\$. 14. II. 2.) kann man schliefsen: Da/s durch die 25 gemeinschaftlichen Puncte der beiden Curven $C^{5}$ und $C_{1}^{5}$ allemal noch eine solche dritte Curve gleichen Grads, etwa $C_{0}^{5}$, geht, welche den Pol $\boldsymbol{P}$ zum Mittelpunct und somit zugleich zum Wendepunct hat. Und dafs ferner, wenn man sich zwischen denselben zwei Curven $C^{5}$ und $C_{1}^{5}$ die durch den $\boldsymbol{P o l} \boldsymbol{P}$ gehenden 25 Wechselsehnen bb $b_{1}$ denkt, von deren Endpuncten nümlich der eine in der einen und der andere in.der andern Curve, die Milte der Sehne aber in $\boldsymbol{P}$ liegt, alsdann die 50 Endpuncte dieser Sehnen ebenfalls in einer solchen Curve $5^{\text {ten }}$ Grads, etwa $\boldsymbol{B}^{5}$, liegen, uselche $\boldsymbol{P}$ sum Mittelpunct hat.

IV. Bei der Basis $C^{6}$ gehen durch jeden beliebigen Pol $P$ je 15 Sehnen $\boldsymbol{S}$, deren 30 Endpuncte $a$ in der Polare $\boldsymbol{J}^{5}$ liegen, welche den Pol zum Mittel- und zugleich zum Wendepunct hat. Die Basis wird von den $15 S$ noch in andern 15.4 $=60$ Puncten $\alpha$, und die Polare $\boldsymbol{J}^{5}$ wird von den- 
selben, aufser in $\boldsymbol{P}$ selbst, noch in $15 \cdot 2=30$ neuen Puncten $a_{1}$ geschnitten, welche paarweise Gegenpuncte in Rücksicht des Mittelpunctes $\boldsymbol{P}$ sind.

„Durch die 60 Puncte a können Curven $1^{\text {ten }}$ Grads $\boldsymbol{R}^{10}$ gehen." Jede dieser Curven schneidet die $15 S$ in neuen $15 \cdot 6=90$ Puncten $\alpha_{1}$.

,Solche 90 Puncte $\alpha_{1}$ liegen jedesmal mit jenen festen 30 Puncten $a_{1}$ zusammen in iryend einer Curve $9^{\text {ten }}$ Grads $\boldsymbol{R}_{1}^{9}$, welche mit der Polare $J^{5}$ den Wendepunct $\boldsymbol{P}$ sammt der zugehörigen Wendetangente gemein hat." [Berühren die Curven $\boldsymbol{R}_{1}^{9}$ und $\boldsymbol{J}^{5}$ einander im Pol $\boldsymbol{P}$ nicht höher als dreipunctig? Kann die Curve $\boldsymbol{R}_{1}^{9}$ nicht in $\boldsymbol{C}_{1}^{6}+\boldsymbol{C}^{3}$ zerfallen? Oder können durch die 30 Puncte $a_{1}$ nicht auch Curven $6^{\text {ten }}$ Grads, $C_{1}^{6}$, gehen? Jede solche $C_{1}^{6}$ schnitte alsdann die $15 S$ in neuen 60 Puncten $\alpha^{\prime}$, und diese $60 \alpha^{\prime}$ lägen mit den festen $60 \alpha$ in einer Curve $\boldsymbol{R}^{10}$, welche die $15 S$ in noch $15 \cdot 2=30$ andern Puncten $\alpha^{\prime \prime}$ schnitte, und diese $30 \alpha^{\prime \prime}$ müfsten sodann in einer Curve $\boldsymbol{C}^{3}$ liegen, welche mit $\boldsymbol{J}^{5}$ den Wendepunct $\boldsymbol{P}$ nebst der zugehörigen Wendetangente gemein hätte, oder sie noch höher berührte.]

V. Bei der Basis $C^{7}$ gehen durch jeden Pol $P$ je 21 Sehnen $S$, deren 42 Endpuncte $\boldsymbol{a}$ in der Polare $\boldsymbol{J}^{6}$ liegen, die den Pol zum Mittelpunct hat. Die Basis wird von den $21 S$ noch in $21.5=105$ Puncten $\alpha$, und die Polare wird von denselben noch in $21 \cdot 4=84$ Puncten $a_{1}$ geschnitten. Die in jeder Sehne $\boldsymbol{S}$ liegenden 4 Puncte $a_{1}$ bestehen aus zwei Paar Gegenpuncten in Rücksicht der Polare $\boldsymbol{J}^{6}$ und ihres Mittelpunctes $\boldsymbol{P}$.

„Durch die 105 Puncte a können Curven $1^{\text {ten }}$ Grads, $\boldsymbol{R}^{15}$, gehen." Jede dieser Curven schneidet die $21 S$ in neuen $21 \cdot 10=210$ Puncten $\alpha_{1}$, und

„Solche 210 Puncte $\alpha_{1}$ liegen jedesmal, mit jenen festen 84 Puncten $a_{1}$ zusammen, in irgend einer Curve 14 ${ }^{\text {ten }}$ Grads $R_{1}^{14} . "$ In Rücksicht dieser Curven und des Pols findet das Eigenthümliche statt: „Dafs die innere Polare, $J_{1}^{13}$, des Pols $P$ in Bezug auf jede Curve $R_{1}^{14}$ in zurei Theile zerfällt, uovon der eine allemal die vorgenannte Polare $J^{6}$, der andere aber irgend eine Curve $J^{7}$ ist, welche gleichfalls den Pol sum Mittelpunct (und zugleich sum Wendepunct) hat." - [Können hierbei nicht auch an die Stelle der Curve $\boldsymbol{R}_{1}^{14}$ zwei solche Curven $7^{\text {ten }}$ Grads, elwa $\boldsymbol{C}_{1}^{7}+\boldsymbol{C}_{2}^{7}$, treten, wovon die eine, in Rücksicht der zwei Paar Gegenpuncte $a_{1}$ in jeder Sehne $\boldsymbol{S}$, je durch das eine und die andere durch das jedesmalige andere Paar geht? Welche Paare, in Betracht aller 21 Sehnen, gehören zusammen, oder wieviele Änderungen sind dabei möglich? U. s. w.] 
70 2. St einer, algeb. Curven, welche Mittelp. haben, u. Eigens. allgem. algeb.Curven.

Analoge Eigenschaften, wie bei den beiden letzten Beispielen (IV.) und (V.), finden auch bei den allgemeinen Basen $C^{2 \mu}$ und $C^{2 v-1}$ statt.

Innere Panpolaren.

S. 21.

I. Ist in einer Ebene ein Curven-Büschel $m^{\text {ten }} \mathrm{Grads}, \boldsymbol{B}\left(\boldsymbol{C}^{m}\right)$, mit $\boldsymbol{m}^{2}$ Grundpuncten $\boldsymbol{p}$ gegeben (Monatsber.), so gehen durch jeden in der Ebene beliebig gewählten Pol $\boldsymbol{P}$, rücksichtlich jeder einzelnen Curve $\boldsymbol{C}^{m}$, ein bestimmtes System von $\frac{1}{2} m(m-1)$ Sehnen $S$, deren $m(m-1)$ Endpuncte $a$ in der zugehörigen innern Polare $J^{m-1}$ liegen.

„Alle innern Polaren, die demselben Pol $\boldsymbol{P}$ in Bezug auf die einzelnen Curven des gegebenen Curven-Büschels $B\left(C^{m}\right)$ entsprechen, bilden unter sich gleicherweise einen Curven-Büschel $\boldsymbol{B}\left(\boldsymbol{J}^{m-1}\right)$ mit $(\boldsymbol{m}-1)^{2}$ Grundpuncten $q$ und haben den Pol sum gemeinsamen Mittelpunct; sudem sind ihre Grundpuncte $q$ paarweise Gegenpuncte, etwa q und $q_{1}$, in Rücksicht des Mittelpunctes $\boldsymbol{P}$, so da/s also die Polaren sämmtlich $\frac{1}{2}(m-1)^{2}$ Sehnen $q \eta_{1}$ gemein haben. In dem Falle, wo $m-1$ und damit auch $(\boldsymbol{m}-1)^{2}$ ungerade ist, liegt der unpaare oder einzelne Punct $\boldsymbol{q}$, der $\boldsymbol{q}_{0}$ heifsen mag, im Pol $\boldsymbol{P}$ selbst, und die Zahl der Sehnen $q q_{1}$ wird dabei nur durch die in dem Ausdrucke $\frac{1}{2}(m-1)^{2}$ enthaltene ganze Zahl angezeigt.

„Die gesummten Asymptoten $\boldsymbol{A}_{s}$ aller gegebenen Curven $\boldsymbol{B}\left(\boldsymbol{C}^{m}\right)$ umhüllen eine Curve $(2 m-1)^{\text {ster }}$ Classe $A_{s}^{2 m-1}$ und $4(m-1)^{\text {sten }}$ Grads, welche die Gerade $G_{\infty}$ zur $2(m-1)$ fachen T'angente hat; so dafs also durch jeden gegebenen Pol $\boldsymbol{P}$, im Allgemeinen, 2m-1 Asymptoten $\boldsymbol{A}_{s}$ gehen, dagegen aber nach jedem in $G_{\infty}$ liegenden Puncte $Q$, oder nach jeder gegebenen Richtung nur je eine Asymptote $\boldsymbol{A}_{s}$ geht, somit keine zwei parallel sein können." *)

Werden die Endpuncte aller jener Systeme Sehnen, welche demselben Pol in Bezug auf alle gegebenen Curven entsprechen, zusammengefafst, so ergiebt sich der folgende Satz.

*) „Werden die gegebenen Curven $\boldsymbol{B}\left(\boldsymbol{C}^{m}\right)$ von eiver beliebigen Geraden $\boldsymbol{G}$ geschnitten und man denkt sich in den Schnittpuncten an dieselben Tangenten $A$ gelegt, so umhüllen diese Tangenten gleicherweise eine Curve $(2 m-1)^{\text {ster Classe } A^{2 m-1} \text { und }}$ $4(m-1)^{\text {sten }}$ Grads, welche die Gerade $G$ zur $2(m-1)$ fachen Tangente hat." Dieser Satz ist der Abhandlung entnommen, welche der obige Monatsber. bespricht. 
9. St einer, algeb. Curven, welche Mittelp. haben, u. Eigens. allgem. algeb. Curven. 71

„Die Endpuncte a aller Systeme Sehnen $\mathbf{S}$, die irgend einem und demselben Pol $\boldsymbol{P}$ in Betracht aller einzelnen Curven des gegebenen Curven-Büschels $\boldsymbol{B}\left(C^{m}\right)$ sugehören, liegen zusammen in einer Curve $(2 m-1)^{s t e n}$ Grads, $J^{2 m-1}$, welche den Pol $\boldsymbol{P}$ zum Mittelpunct und die durch denselben gehenden $2 m-1$ Asymptoten $A_{s}$ von einzelnen der gegebenen Curven auch selbst zu Asymptoten hat, so da/s sie diese Curven in den unendlich entfernten Puncten $a_{\infty}$ der respectiven Asymptoten berührt, und welche ferner sowohl durch die $m^{2}$ Grundpuncte $p$ des gegebenen Curven-Büschels, als auch durch die $(m-1)^{2}$ Grundpuncte $q$ des Büschels innere Polaren, $\boldsymbol{B}\left(J^{m-1}\right)$, desselben Pols in Bezug auf jenen gegebenen Curven-Büschel geht." Diese Curve $J^{2 m-1}$ soll ,innere Panpolare" des Pols $\boldsymbol{P}$ in Bezug auf den gegebenen Curven-Büschel $\boldsymbol{B}\left(\boldsymbol{C}^{m}\right)$ genannt werden. Da dieselbe immer von ungeradem Grad ist, $2 m-1$, so geht sie stets durch ihren eigenen Mittelpunct $\boldsymbol{P}$ und hat ihn zugleich zum Wendepunct.

Unter den unendlich vielen Sehnen $\boldsymbol{S}$, welche in Betracht aller gegebenen Curven durch den jedesmaligen Pol $\boldsymbol{P}$ gehen, giebt es allemal einzelne solche besondere Sehnen, welche in ihrem einen Endpuncte die zugehörige Curve berühren, statt schneiden. Jede solche Tangenten-Sehne soll durch $\boldsymbol{S}_{0}$ und ihre Endpuncte durch $a$ und $a_{0}$, nämlich der genannte Berührungspunct durch $a_{10}$ bezeichnet werden. (In speciellem Falle können beide Endpuncte Berührungspuncte werden.) Ist $\boldsymbol{S}_{0}$ insbesondere eine Asymptote $\boldsymbol{A}_{s}$ der zugehörigen Curve $\boldsymbol{C}^{m}$, so ist $\boldsymbol{a}_{0}$ nicht mehr allein als Berührungspunct anzusehen, sondern in diesem Falle hat man sich auch $a$ in dem unendlich entfernten Berührungspuncte $a_{\infty} \operatorname{der} \boldsymbol{A}_{s}$ zu denken, und zwar $a_{0}$ nach der einen und $a$ nach der entgegengesetzten Richtung, so dafs beide vereint den Berührungspunct $a_{\infty}$ bilden, und dennoch gleichweit vom Pol $\boldsymbol{P}$ abstehen. Demnach ist jede Asymptote $\boldsymbol{A}_{s}$ einer Curve $\boldsymbol{C}^{m}$ in Rücksicht jedes in ihr angenommenen Pols $P$ allemal als eine Tangenten-Sehne $\mathcal{S}_{0}$ anzusehen, deren beide Endpuncte a und $a_{0}$ jedoch als in ihrem Berührungspuncte $a_{\infty}$ vereinigt, aber als nach entgegengesetzten Richtungen liegend $\approx u$ betrachten sind.

„Durch jeden Pol P gehen, im Allgemeinen, $3 m(m-2)+2 m-1$ Tangenten-Sehnen $\boldsymbol{S}_{0}$, die jedoch von wweierlei Art sind; nämlich: 1) $2 m-1$ derselben bestehen aus den vorgenunnten, durch den Pol $P$ gehendeu Asymptoten $A_{s}$ einselner gegebener Curven (den Asymptoten 
72 2. Steiner, algeb. Curven, welche Mittelp. haben, u. Eigens. allgem. algeb. Curven.

der Panpolare), so da/s ihre Endpuncte in den respectiven Berührungspuncten $a_{\infty}$ vereint liegen; 2) dagegen sind die $3 m(m-2)$ übrigen eigentliche Tangenten-Sehnen $\boldsymbol{S}_{0}$, deren Endpuncte a und $a_{0}$ verschieden und nicht im Unendlichen liegen; die $3 m(m-2)$ Berührungspuncte $a_{0}$ dęr letstern liegen allemal mit den $m^{2}$ Grundpuncten $p$ des gegebenen Curven-Büschels zusammen in irgend einer Curve $2(m-1)^{\text {sten }}$ Grads, $A_{0}^{2 m-2}$, welche die zugehörige Panpolare $J^{2 m-1}$ in ihrem Mittelpuncte $P$ berührt." Die Curven $A_{0}^{2 m-2}$ und $J^{2 m-1}$ schneiden einander in den $3 m(m-2)$ Puncten $a_{0}$, so wie in den $m^{2}$ Puncten $p$ und berühren sich in $P$ zweipunctig; wras zusammen gerade die volle Zahl ihrer gemeinschaftlichen Puncte $2(m-1)(2 m-1)$ ausmacht.

Eine gegebene Curve $\boldsymbol{C}^{m}$ werde von einer Tangente $\boldsymbol{S}_{0}$ im Puncte $\boldsymbol{a}_{0}$ berührt und nebstdem in $m-2$ Puncten $a$ geschnitten; man bezeichne die Mitte jeder Strecke $a a_{0}$ durch $\mathfrak{M}$, so hat man in jeder Tangente $m-2$ Puncte $\mathfrak{M}$ und der Ort aller dieser Puncte wird irgend eine Curve $x^{\text {ten }}$ Grads, $\mathfrak{M}^{x}$, sein, welche jedoch aus den $m$ Asymptoten $\boldsymbol{A}_{s}$ der gegebenen Curve $\boldsymbol{C}^{m}$ und aus einer eigentlichen Curve $(x-m)^{\text {ten }}$ Grads, $\mathfrak{M}^{x-m}$, besteht, so dafs, wenn man $x-m=y$ setzt,

$$
\mathfrak{M}^{x}=\mathfrak{M}^{y}+\boldsymbol{m} \cdot \boldsymbol{A}_{s}
$$

ist. So gehört also zu jeder Curve $C^{m}$ eine Ortscurve $\mathfrak{M}^{y}$.

„Die zu allen Curven eines gegebenen Curven-Büschels $\boldsymbol{B}\left(\boldsymbol{C}^{m}\right)$ gehörige Schaur Ortscurven $\boldsymbol{S}\left(\mathfrak{M}^{y}\right)$ bedecken die ganze Ebene der.gestalt, da/s durch jeden Pol P, im Allgemeinen, $3 m(m-2)$ derselben gehen." Weil nämlich eben so viele Tangenten-Sehnen durch jeden Pol gehen, aufser den $2 m-1$ Asymptoten. In dem speciellen Falle, wo $m=3$, ist $y=15$, und: Bei einesn gegebenen Curven-Büschel $\boldsymbol{3}^{\text {ten }}$ Grads, $\boldsymbol{B}\left(\boldsymbol{C}^{3}\right)$, gehen von der Schaar zugehörigen Ortscurven $\boldsymbol{S}\left(\mathfrak{M}^{15}\right)$ durch jeden Pol $\boldsymbol{P}$ je 9 , so wie nebstdem je 5 Asymptoten $\boldsymbol{A}_{s}$ von einzelnen der gegebenen Curven. [1. Den Grad, $y$, der Ortscurve $\mathfrak{M}^{y}$ allgemein zu bestimmen. 2. Die Enveloppe der $\boldsymbol{S}\left(\mathfrak{M}^{y}\right)$ zu finden.]

Unter den durcb irgend einen $\mathrm{Pol} \boldsymbol{P}$ gehenden unendlich vielen Sehnen $\boldsymbol{S}$ der gegebenen Curven $\boldsymbol{B}\left(\boldsymbol{C}^{m}\right)$ giebt es ferner auch solche besondere, die $\mathfrak{S}$ statt $\boldsymbol{S}$ heifsen sollen, in deren Endpuncten $\mathfrak{a}$ und $\mathfrak{a}_{1}$ die Tangenten $\mathfrak{A}$ und $\mathfrak{A}_{1}$ der zugehörigen Curve $\mathfrak{S}^{m}$ (statt $C^{m}$ ) parallel sind, und wobei also diese Curve in den Endpuncten der Sehne sowohl von ihrer innern Polare $\mathfrak{\Im}^{m-1}$ (statt $J^{m-1}$ ) als auch von der Panpolare $J^{2 m-1}$ berührt wird. Es giebt für 
2. Stein er, algeb. Curven, welche Mittelp. haben, u. Eigens. allgem. algeb. Curven. 73 jeden Pol $\boldsymbol{P}$, im Allgemeinen, $(3 m-1)(\boldsymbol{m}-2)+\frac{1}{2}$ solche besondere Sehnen $\mathfrak{S}$, oder im engeren Sinne nur $(3 m-1)(m-2)$, indem der Bruch, $\frac{1}{2}$, die Tangente der durch $\boldsymbol{P}$ gehenden Curve $\boldsymbol{C}^{\prime n}$ anzeigt, bei welcher $\mathfrak{a}$ und $\mathfrak{a}_{1}$ sich in $\boldsymbol{P}$ vereinigt haben und die genannten drei Curven einander nur noch in diesem einen Puncte $\boldsymbol{P}$ berühren. Als dergleichen uneigentliche Sehnen $\mathfrak{S}$ machen sich ferner auch die durch $\boldsymbol{P}$ gehenden 2 in -1 Asymptoten $\boldsymbol{A}_{s}$ geltend, in deren Puncten $\boldsymbol{a}_{\infty}$ die zugehörigen beiden Curven $\boldsymbol{C}^{m}$ und $\boldsymbol{J}^{m-1}$ einander ebenfalls berühren und zugleich von der Panpolare berührt werden. Aufserdem berührt die Panpolare $J^{2 m-1}$ in jedem der $\boldsymbol{n}^{2}$ Grundpuncte $\boldsymbol{p}$ irgend eine Curve $\boldsymbol{C}^{m}$, aber nicht auch zugleich deren Polare $\boldsymbol{J}^{m-1}$; und eben so berührt dieselbe in jedem der $(\boldsymbol{m}-1)^{2}$ Puncte $q$ irgend eine der Polaren $\boldsymbol{B}\left(\boldsymbol{J}^{m-1}\right)$, aber nicht auch deren Basis $C^{m}$. Also:

„Durch jeden Pol P gehen, im Allgerneinen, $(3 m-1)(m-2)$ eigent-

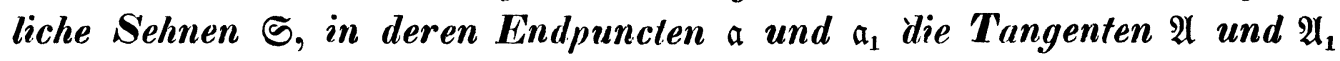
parallel sind und die jedesmalige Curve $\mathfrak{S}^{m}$ sowohl von ihrer innern Polare $\mathfrak{J}^{m-1}$ als auch von der Panpolare $\boldsymbol{J}^{2 m-1}$ berührt wird." Oder: ,Unter den jedem Pol $\boldsymbol{P}$ entsprechenden innern Polaren $\boldsymbol{B}\left(J^{m-1}\right)$ giebt es je $(3 m-1)(m-2)$ solche, $\mathfrak{\Im}^{m-1}$, welche ihre Basis, $\mathfrak{S}^{m}$, in zwei Puncten berühren." Oder: „Die Panpolare $J^{2 m-1}$ jedes Pols $P$ berührt je $(3 m-1)(m-2)$ der yegebenen Curven $B\left(C^{m}\right)$ in je swei Puncten a und $\mathfrak{a}_{1}$, welche stets Gegenpuncte in Rücksicht des Pols sind, und sobald dieselbe eine der gegebenen Curven in irgend einem Puncte a berührt, der weder in der Geraden $G_{\infty}$ liegt, noch einer der $m^{2}$ Puncte $p$ ist, so berührt sie dieselbe nothwendig noch in einem andern Puncte $\mathfrak{a}_{1}$, und zuar im Gegenpuncte von jenem in Rücksicht uuf den Pol P." Dabei kann, in Betracht einer einzelnen gegebenen Curve, auch noch das bemerkt werden: „Liegt der Pol $\boldsymbol{P}$ in einer Asymptole $\boldsymbol{A}_{s}$ einer Curve $\boldsymbol{C}^{m}$, so beruhrt seine innere Polare $J^{m-1}$ die letstere im unendlich entfernten Puncte $a_{\infty} \operatorname{der} \boldsymbol{A}_{s}$."

In jeder einzelnen Curve $\boldsymbol{C}^{m}$ müssen alle solche Sehnen $\mathfrak{S}$, in deren Endpuncten $\mathfrak{a}$ und $\mathfrak{a}_{1}$ die Tangenten $\mathfrak{A}$ und $\mathfrak{A}_{1}$ parallel sind, irgend eine Curve $y^{\text {ter }}$ Classe, $\mathfrak{S}^{y}$, umhüllen, und eben so mufs, wenn man die Mitte jeder Sehne $\mathfrak{S}$ durch $\mathfrak{N}$ bezeichnet, der Ort aller $\mathfrak{N}$ irgend eine Curve $x^{\text {ten }}$ Grads, $\mathfrak{N}^{x}$, sein. In dieser Hinsicht gehören alsdann zu den Curven des gegebenen Büschels $\boldsymbol{B}\left(\boldsymbol{C}^{m}\right)$ zwei Schaaren Ortscurven, $\boldsymbol{S}\left(\mathfrak{S}^{y}\right)$ und $\boldsymbol{S}\left(\mathfrak{R}^{x}\right)$.

Crelle's Journal t. d. M. Bd. XLVlI. Heft 1. 
74 2. St einer, algeb. Curven, welche Mittelp. haben, u. Eigens. allgem. algeb. Curven.

„Die Schaar Ortscurven $\boldsymbol{S}_{\left(\mathfrak{R}^{x}\right)}$, welche zu dern gegebenen Curven-Büschel $\boldsymbol{B}\left(C^{m}\right)$ gehören, überziehen die ganze Ebene dergestalt, da/s durch jeden beliebigen Pol P, im Allgemeinen, je $(3 m-1)(m-2)$ derselben yehen." Für den speciellen Fall, wo $m=3$, sind nach dem Obigen (\$. 15.) die Curven $\mathfrak{S}^{y}$ und $\mathfrak{N}^{x}=\mathfrak{S}^{9}$ und $\mathfrak{N}^{12}$, und in Rücksicht des CurvenBüschels $\boldsymbol{B}\left(C^{3}\right)$ gehen durch jeden Punct $\boldsymbol{P}$ der Ebene je 8 Ortscurven $\mathfrak{N}^{12}$. [1. Die Classe $y$ und den Grad $x$ der beiden Ortscurven allgemein zu bestimmen. 2. Die Enveloppen von $\boldsymbol{S}\left(\mathfrak{S}^{y}\right)$ und $\boldsymbol{S}\left(\mathfrak{R}^{x}\right)$ zu finden.]

Wird mit der innern Panpolare zugleich auch die äufsere Panpolare $\boldsymbol{A}^{2 m-1}$ (Monatsber.) desselben Pols in Bezug auf den nämlichen gegebenen Curven-Büschel betrachtet, so findet sich folgende gegenseitige Beziehung derselben (vergl. \$. 13. II.):

„Die beiden Panpolaren $A^{2 m-1}$ und $J^{2 n-1}$ jedes Pols $P$ in Bezug auf den gegebenen Curven-Büschel $B\left(C^{m}\right)$ haben mit der Geraden $G_{\infty}$ die nämlichen 2un-1 Puncte $a_{\infty}$ gemein, so dafs ihre Asymptoten paurweise parallel sind; ferner berühren sie einander im Pol $\boldsymbol{P}$ und ihre übrigen gemeinschaftlichen Puncte bestehen aus den $m^{2}$ Grundpuncten $p$ und aus den obigen $3 m(m-2)$ Berührungspuncten $a_{0}$ der sogenannten TangentenSehnen $\mathbf{S}_{10}$; was zusammen richtig die volle Zahl ihrer gemeinschaftlichen Puncte, $(2 m-1)(2 m-1)$, beträgt." Wird der Pol P insbesondere in einem der Grundpuncte $p$ angenommen, so wird er ein dreifacher Punct von jeder der beiden Panpolaren, und zwar berühren die durch ihn gehenden beidseitigen drei Curven-Zuveige einander paarweise in ihm."

II. Rücksichtlich des Büschels innere Polaren $\boldsymbol{B}\left(J^{m-1}\right)$ sind, unter andern, noch folgende Umstände beachtenswerth.

Da die Curven irgend eines Büschels $\boldsymbol{x}^{\text {ten }} \mathrm{Grads}, \boldsymbol{B}\left(\boldsymbol{C}^{x}\right)$, insgesammt $3(x-1)^{2}$ Doppelpuncte haben, und da sich unter denselben je $2(x-1)$ solche befinden, welche irgend eine gegebene Gerade $\boldsymbol{G}$ berühren (Monatsber.): so müssen also auch die Polaren $\boldsymbol{B}\left(J^{m-1}\right)$ jedes Pols $P$ in Bezug auf den gegebenen Curven-Büschel $\boldsymbol{B}\left(C^{\prime \prime}\right)$ allemal im Ganzen $3(m-2)^{2}$ Doppelpuncte enthulten, und es müssen je $2(m-2)$ derselben jede gegebene Gerade, also namentlich auch die Gerade $\boldsymbol{G}_{\infty}$ berühren.

Daraus ist zunächst zu entnehmen, dafs es in Betracht jeder einzelnen Curve $\boldsymbol{C}^{m}$ auch solche Pole $\boldsymbol{P}$ geben mufs, deren innere Polaren $\boldsymbol{J}^{m-1}$ Doppelpuncte haben; und zwar müssen diese Doppelpuncte, im Allgemeinen, paarweise vorhanden sein, nämlich als Gegenpuncte in Rūcksicht des Pols oder 
2. St einer, algeb. Curven, welche Mittelp. haben, u. Eigens. allgem. algeb. Curven. 75

Mittelpuncts $\boldsymbol{P}$ der jedesmaligen Polare $\boldsymbol{J}^{m-1}$ (\$. 9. II.); nur wenn ein Doppelpunct insbesondere im Mitlelpunct $\boldsymbol{P}$ selbst, oder wenn er in der Geraden $\boldsymbol{G}_{\infty}$ liegt, steht er als einzeln da. Von jeder Curve, etwa $\boldsymbol{J}_{1}^{m-1}$, welche einen Doppelpunct $\mathfrak{p}_{\infty}$ auf der Geraden $\boldsymbol{G}_{\infty}$ hat, kann man sagen, sie berühre diese Gerade in demselben; und wenn die Curve einen Mittelpunct $\boldsymbol{P}_{1}$ hat, so kann man umgekehrt behaupten: sie könne die Gerade $\boldsymbol{G}_{\infty}$, im Allgemeinen, nur in diesem Sinne berühren, dafs sie einen auf derselben liegenden Doppelpunct hat. Da nun aber mit der innern Polare $J_{1}^{m-1}$ auch zugleich die äufsere Polare $\boldsymbol{A}_{1}^{m-1}$ desselben Pols $\boldsymbol{P}_{1}$ die Gerade $\boldsymbol{G}_{\infty}$ im nämlichen Puncte berührt, so folgt also:

„Da/s der Ort desjenigen Pols $P_{1}$, dessen innere Polare $J_{1}^{m-1}$ in Bezug auf die gegebene Basis $C^{m}$ die Gerade $G_{\infty}$ berührt und somit einen auf ihr liegenden einzelnen Doppelpunct $\mathfrak{p}_{\infty}$ hat, eine bestinmte Curve $2(m-2)^{\text {ten }}$ Grads $\boldsymbol{P}_{1}^{2 m-4}$ und $(m-1)^{\text {ster }}$ Classe $\boldsymbol{D}^{m-1}$ ist; nämlich diese Curve ist die $(m-1)^{\text {ste }}$ Polare der Geraden $G_{\infty}$ in Bezug auf die Basis $C^{m}$, oder die Enveloppe aller Durchmesser $\boldsymbol{D}$ der letztern."

In den Mittelpunct oder Pol $\boldsymbol{P}$ kommt vornebmlich dann ein einzelner Doppelpunct der Polare zu liegen, wenn $m$ ungerad, $=2 v-1$, ist und $\boldsymbol{P}$ in der Basis $C^{m}=C^{2 v-1}$ selbst liegt, so dafs also für diesen Fall die Basis als Hauptbestandtheil seines Ortes anzusehen ist. Dabei sind alsdann die gegenseitigen Schnitte der beiden Curven $\boldsymbol{P}_{1}^{2 m-4}$ und $\boldsymbol{C}^{m}$ solche besondere Pole $\boldsymbol{P}$, deren Polaren zwei vereinzelte Doppelpuncte, $\mathfrak{P}_{\infty}$ und $\boldsymbol{P}$, haben. Übrigens kann der Mittelpunct $\boldsymbol{P}$ insbesondere auch ein mehr als zweifacher Punct der Polare $J^{m-1}$ werden, jedoch nur nach Mafsgabe der zwei Zahlformen von $\boldsymbol{m}$, nur so, wie bereits oben ( $\$$. 1.) angegeben worden.

Aufser diesen speciellen Fällen, wobei die Polare einen vereinzelten Doppelpunct hat, mufs es nun, in Bezug auf die gegebene Basis $C^{m}$, auch solche Pole geben, die $\boldsymbol{P}_{2}$ heifsen sollen, deren innere Polaren $\boldsymbol{J}_{2}^{m-1}$ ein Paar (oder insbesondere auch mehre Paare) Doppelpuncte, $\mathfrak{p}_{2}$ und $\mathfrak{p}_{2}^{\prime}$, haben, welche dann stets Gegenpuncte in Rücksicht des Pols $\boldsymbol{P}_{2}$ sind; und zwar mufs der Ort aller solcher Pole irgend eine Curve $x^{\text {ten }}$ Grads $\boldsymbol{P}_{2}^{x}$ sein.

Hiernach gehören also zu der gegebenen Curve $C^{m}$, im Allgemeinen, zwei solche Ortscurven $\boldsymbol{P}_{1}^{2 m-4}$ und $\boldsymbol{P}_{2}^{x}$, wovon die erste alle diejenigen Pole $\boldsymbol{P}_{1}$ enthält, deren Polaren $\boldsymbol{J}_{1}^{m-1}$ einen auf der Geraden $\boldsymbol{G}_{\infty}$ liegenden einzelnen Doppelpunct haben, die andere, unbekannte Curve dagegen alle diejenigen Pole $\boldsymbol{P}_{2}$ enthält, deren Polaren $\boldsymbol{J}_{2}^{m-1}$ Paare von Doppelpuncten haben. Die 
$2(m-2) \times x$ gemeinschaftlichen Puncte beider Ortscurven sind solche besondere Pole, welche beide Eigenschaften zugleich besitzen. In dem Falle, wo $m=2 v-1$, hat ferner auch jede innere Polare, deren Pol in der Basis $C^{2 v-1}$ selbst liegt, in diesem Pol einen einzelnen Doppelpunct.

In dieser Hinsicht gehören demnach zu allen Curven eines gegebenen Curven-Büschels $\boldsymbol{B}\left(\boldsymbol{C}^{m}\right)$, im Allgemeinen, sowohl eine Schaar Ortscurven $\boldsymbol{P}_{1}^{2 m-4}$, oder $\boldsymbol{S}\left(\boldsymbol{P}_{1}^{2 m-4}\right)$, als auch eine Schaar Ortscurven $\boldsymbol{P}_{2}^{x}$, oder $\boldsymbol{S}\left(\boldsymbol{P}_{2}^{x}\right)$.

Da nun unter dem Büschel Polaren $\boldsymbol{B}\left(\boldsymbol{J}^{m-1}\right)$ jedes Pols $\boldsymbol{P}$ in Bezug auf den gegebenen Curven-Büschel $\boldsymbol{B}\left(\boldsymbol{C}^{m}\right)$ sich $\mathbf{2}(\boldsymbol{m}-\mathbf{2})$ solche befinden, welche die Gerade $G_{\infty}$ berühren, also $2(m-2)$ solche Polaren $J_{1}^{m-1}$, welche einzelne Doppelpuncte $\mathfrak{p}_{\infty}$ auf $\boldsymbol{G}_{\infty}$ haben; und da der Büschel $\boldsymbol{B}\left(\boldsymbol{J}^{m-1}\right)$ im Ganzen $3(m-2)^{2}$ Doppelpuncte enthält, so bleiben also noch

$$
3(m-2)^{2}-2(m-2)=(m-2)(3 m-8)
$$

solche Doppelpuncte übrig, welche nicht im Unendlichen liegen, und welche somit paarweise einzelnen Polaren $\boldsymbol{J}_{2}^{m-1}$ angehören mũssen. Die Anzahl dieser Polaren $\boldsymbol{J}_{2}^{m-1}$ ist daher

$$
=\frac{1}{2}(m-2)(3 m-8),
$$

insofern nämlich diejenigen unter ihnen, welche insbesondere mehre Paare Doppelpuncte haben, ebenso oft gezählt werden, als sie Paare enthalten. Für den Fall, wo $m=2 v-1$, ist die Zahl der Polaren $\boldsymbol{J}_{2}^{m-1}$,

$$
=2(\nu-2)(3 \nu-4)+\frac{1}{2} \text {, }
$$

wo der Bruch $\frac{1}{2}$ diejenige besondere Polare, etwa $J_{0}^{m-1}$ anzeigt, welche einen einzelnen Doppelpunct im Pol $\boldsymbol{P}$ selbst hat, und welche der durch $\boldsymbol{P}$ gehenden Curve $C^{2 v-1}\left(=C^{m}\right)$ zugehört.

Diesen Angaben gemäfs ist nun auch bestimmt, wieviele von jenen genannten Ortscurven durch den jedesmaligen Pol gehen, nämlich:

„Die zu dem gegebenen Curven-Büschel $\boldsymbol{B}\left(\boldsymbol{C}^{m}\right)$ gehörigen zwei Schaaren Ortscurven, $\boldsymbol{S}\left(\boldsymbol{P}_{1}^{2 m-4}\right)$ und $\boldsymbol{S}\left(\boldsymbol{P}_{2}^{x}\right)$, bedecken die ganze Ebene in der Art, da/s durch jeden beliebigen Punct $\boldsymbol{P}$, ion Allgemeinen,

$$
2(m-2) \text { Curven } \boldsymbol{P}_{1}^{2 m-4} \text { und } \frac{1}{2}(m-2)(3 m-8) \text { Curven } \boldsymbol{P}_{2}^{x}
$$

gehen." Wenn $m=2 v-1$, und danach die letzte Zahl $=2(\nu-2)(3 v-4)+\frac{1}{2}$ ist, so gehen $2(\nu-2)(3 \nu-4)$ Curven $\boldsymbol{P}_{2}^{x}$ durch $\boldsymbol{P}$ und der Bruch $\frac{1}{2}$ zeigt diejenige unter den gegebenen Curven an, welche durch $\boldsymbol{P}$ geht.

Es wird zur Erläuterung dienen und auch an sich nicht ohne Interesse sein, wenn wir die ausgesprochenen allgemeinen Eigenschaften bei den ein- 
2. St einer, algeb. Curven, welche Mittelp. haben, u. Eigens. allyem. algeb. Curven. 77

fachsten Beispielen, wo der gegebene Curvenbüschel nur vom $3^{\text {ten }}, 4^{\text {ten }}$ und $5^{\text {ten }}$ Grad ist, etwas näher betrachten.

A. Bei einem gegebenen Curven-Büschel $3^{\text {ten }} \mathrm{Grads}, \boldsymbol{B}\left(\boldsymbol{C}^{3}\right)$, finden keine Ortscurven $\boldsymbol{P}_{2}^{x}$ statt, was auch der Ausdruck $2(\nu-2)(3 \nu-4)$ richtig anzeigt, indem er $=0$ wird, wenn $\nu=2$ ist; wogegen der Bruch $\frac{1}{2}$ bleibt und die durch den Pol $\boldsymbol{P}$ gehende Curve $\boldsymbol{C}^{3}$ anzeigt. Jede der andern Ortscurven $\boldsymbol{P}_{1}^{2 m-4}$ wird hier ein Kegelschnitt $\boldsymbol{P}_{1}^{2}$, und zwar derselbe, welcher schon oben (\$. 15.) betrachtet und durch $\boldsymbol{E}^{2}$ bezeichnet worden. Die innern Polaren jedes Pols $\boldsymbol{P}$ in Bezug auf den gegebenen Curven-Büschel $\boldsymbol{B}\left(\boldsymbol{C}^{\mathbf{3}}\right)$ bilden einen Kegelschnitt-Büschel $\boldsymbol{B}\left(\boldsymbol{J}^{2}\right)$ mit 4 Grundpuncten $q$; dieselben enthalten im Ganzen $3(3-2)^{2}=3$ Doppelpuncte, von welchen $2(3-2)=2$ auf der Geraden $G_{\infty}$ liegen, und einzelne Doppelpuncte zweier besondern Polaren $\boldsymbol{J}_{1}^{2}$ sind, der dritte dagegen liegt im Pol $\boldsymbol{P}$ selbst und ist Doppelpunct der besondern Polare $\boldsymbol{J}_{0}^{2}$, welche der durch $\boldsymbol{P}$ gehenden Curve $\boldsymbol{C}^{3}$ entspricht. Jede der beiden Polaren $\boldsymbol{J}_{1}^{2}$ besteht aus zwei parallelen Geraden $\boldsymbol{J}$ und $\boldsymbol{J}_{1}$, die gleichweit vom Pol $\boldsymbol{P}$ abstehen, und die Polare $\boldsymbol{J}_{0}^{2}$ besteht aus zwei sich in $P$ schneidenden Geraden (Sehnen) $S$ und $S_{1}$ (\$. 15.). Demnach sind die 4 Grundpuncte $q$ des Büschels Polaren $B\left(J^{2}\right)$ allemal die Ecken eines Parallelogramms, welches die Geraden $S$ und $S_{1}$ zu Diagonalen und die zwei Paar Geraden $J$ und $J_{1} \approx u$ Gegenseilen hat; und die zu dem gegebenen Curven-Büschel $\boldsymbol{B}\left(C^{3}\right)$ gehörige Schaar Orts-Kegelschnitte $\boldsymbol{S}\left(\boldsymbol{P}_{1}^{2}\right)$ erfültt die ganze Ebene doppelt, so da/s durch jeden Punct $\boldsymbol{P}_{\text {je }}$ zwei Kegelschnitle $\boldsymbol{P}_{1}^{2}$ gehen.

B. Bei einem gegebenen Curven-Büschel $\boldsymbol{B}\left(\boldsymbol{C}^{4}\right)$ sind die zugehörigen Ortscurven $\boldsymbol{P}_{2}^{x}$ vom $10^{\text {ten }}$ Grad und $36^{\text {ster }}$ Classe (\$. 17.), also $\boldsymbol{P}_{2}^{x}=\boldsymbol{P}_{2}^{\text {10 }}$; die andern Ortscurven $\boldsymbol{P}_{1}^{2 m-4}=D^{m-1}$ sind vom $4^{\text {ten }}$ Grad $\boldsymbol{P}_{1}^{4}$ und $3^{\text {ter }}$ Classe $D^{3}$. Die irgend einem Pol $\boldsymbol{P}$ entsprechenden innern Polaren bilden einen Büschel $\boldsymbol{B}\left(J^{3}\right)$ mit 9 Grundpuncten $q$, und haben im Ganzen $3(4-2)^{2}=12$ Doppelpuncte. Von den 9 Puncten $q$ liegt einer, etwa $q_{0}$, im Pol $\boldsymbol{P}$ selbst und die 8 übrigen bestehen aus 4 Paar Gegenpuncten $q$ und $q_{1}$ rücksichtlich des Pols, so dafs sie die Endpuncte von 4 gemeinschaftlichen Sehnen $S$ der Polaren $\boldsymbol{B}\left(\boldsymbol{J}^{3}\right)$ sind. Von den 12 Doppelpuncten liegen 4 auf der Geraden $\boldsymbol{G}_{\infty}$ und sind einzelne Doppelpuncte von 4 besondern Polaren $\boldsymbol{J}_{1}^{3}$, dagegen sind die 8 übrigen paarweise Doppelpuncte, $\mathfrak{p}_{2}$ und $\mathfrak{p}_{2}^{\prime}$, von vier besondern Polaren $\boldsymbol{J}_{2}^{3}$ und zugleich Gegenpuncte in Rücksicht des Pols $\boldsymbol{P}$. Jede der 4 letztern Polaren $\boldsymbol{J}_{2}^{3}$ mufs aus $\boldsymbol{J}^{2}+\boldsymbol{S}_{2}$ bestehen ( $\$$. 17.), und zwar mufs der Kegelschnitt 
78 2. Steiner, algeb. Curven, welche Mittelp. haben, u. Eigens. allgem. algeb.Curven.

$J^{2}$ durch je drei Paar Grundpuncte $q$ und $q_{1}$, und die Doppelsehne $\boldsymbol{S}_{2}$ mufs durch das jedesmalige vierte Paar gehen, also auf eine der 4 Sehnen $\boldsymbol{S}$ fallen; und da nun das zugehörige Paar Doppelpuncte aus den gegenseitigen Schnitten von $\boldsymbol{J}^{2}$ und $\boldsymbol{S}_{2}$ besteht, so liegen also in jeder der 4 Doppelsehnen $\boldsymbol{S}_{2}$ (oder auch der 4S) sowohl ein Paar Doppelpuncte $\mathfrak{p}_{2}$ und $\mathfrak{p}_{2}^{\prime}$, als auch ein Paar Grundpuncte $q$ und $q_{1}$ des $\boldsymbol{B}\left(\boldsymbol{J}^{3}\right)$, so wie auch jede derselben durch den $9^{\text {ten }}$ Grundpunct $q_{0}$ (oder Pol $\boldsymbol{P}$ ) geht. Von den su dem gegebenen CurvenBüschel $\boldsymbol{B}\left(C^{4}\right)$ gehörigen zwei Schauren Ortscurven, $\boldsymbol{S}\left(\boldsymbol{P}_{1}^{4}\right)$ und $\boldsymbol{S}\left(\boldsymbol{P}_{2}^{10}\right)$, bedeckt jede die ganze Ebene vierfach, d.h. durch jeden Punct $P$ der Ebene gehen sowohl 4 Curven $P_{1}^{4}$ als auch 4 Curven $P_{2}^{10}$. Jede Ortscurve $\boldsymbol{P}_{2}^{10}$ hat 9 dreifache Puncte $\boldsymbol{P}_{3}$ (\$. 17.); der Ort dieser Puncle, rücksichtlich aller Ortscurven $\boldsymbol{S}_{\left(\boldsymbol{P}_{2}^{10}\right)}$, ist eine Curve $6^{\text {ten }}$ Grads $\boldsymbol{P}_{3}^{6}$, welche insbesondere auch durch die zu dem gegebenen Curven-Büschel $B\left(C_{*}^{4}\right)$ gehörigen 27 Doppelpuncte geht.

C. Bei dem gegebenen Curven-Büschel $\boldsymbol{B}\left(\boldsymbol{C}^{5}\right)$ bilden die Polaren jedes Pols $\boldsymbol{P}$ einen Büschel $\boldsymbol{B}\left(J^{4}\right)$ mit 16 Grundpuncten $q$ und im Ganzen mit $3(5-2)^{2}=27$ Doppelpuncten; es befinden sich unter denselben $2(5-2)=6$ solche, $\boldsymbol{J}_{1}^{4}$, welche einen einzelnen Doppelpunct $\mathfrak{p}_{\infty}$ auf der Geraden $\boldsymbol{G}_{\infty}$ haben, und ferner $2(3-2)(3 \cdot 3-4)=10$ solche, $J_{2}^{4}$, welche ein Paar Doppelpuncte $\mathfrak{p}_{2}$ und $\mathfrak{p}_{2}^{\prime}$ haben, die Gegenpuncte rücksichtlich des Pols $\boldsymbol{P}$ sind, und endlich noch eine solche, $\boldsymbol{J}_{0}^{4}$, welche einen einzelnen Doppelpunct im Pol $\boldsymbol{P}$ selbst hat, was zusammen die 27 Doppelpuncte ausmacht. Dem entsprechend gehen also von den zu dem gegebenen Curven-Büschel gehörigen Ortscurven $\boldsymbol{S}\left(\boldsymbol{P}_{1}^{6}\right)$ und $\boldsymbol{S}\left(\boldsymbol{P}_{2}^{x}\right)$ durch jeden Pol $\boldsymbol{P}$ einerseits je 6 Curven $\boldsymbol{P}_{1}^{6}\left(=\boldsymbol{D}^{4}\right)$ und andererseits je 10 Curven $\boldsymbol{P}_{2}^{x}$.

Die 16 Grundpuncte $q$ des Büschels $\boldsymbol{B}\left(\boldsymbol{J}^{4}\right)$ bestehen aus 8 Paar Gegenpuncten $q$ und $q_{1}$, oder sie sind die Endpuncte von 8 gemeinschaftlichen Sehnen $\boldsymbol{S}$ dieses Büschels. Die besondere Polare $\boldsymbol{J}_{0}^{4}$ kann möglicherweise aus $\boldsymbol{J}^{3}+\boldsymbol{S}_{2}$ bestehen ( $\boldsymbol{S}$. 19.), wobei sie alsdann drei Doppelpuncte hat, die gegenseitigen Schnitte von $\boldsymbol{J}^{3}$ und $\boldsymbol{S}_{2}$, wovon der eine, als einzelner, in $\boldsymbol{P}$ liegt und die zwei andern ein Paar Gegenpuncte sind; ferner geht dabei die Curve $J^{3}$ durch 6 der 8 Paar. Grundpuncte $q$ und $q_{1}$ und die beiden übrigen Paare liegen in der Doppelsehne $\boldsymbol{S}_{2}$, so dafs also die letztere zugleich eine gemeinschaftliche Doppelsehne aller Polaren $\boldsymbol{B}\left(\boldsymbol{J}^{4}\right)$ ist. Diese specielle Polare $\boldsymbol{J}^{3}+\boldsymbol{S}_{2}$ vertritt zugleich eine der 10 Polaren $\boldsymbol{J}_{2}^{4}$. Es kann ferner mo̊glicherweise eine der 10 Polaren $J_{2}^{4}$ aus $J^{2}$ und $J_{1}^{2}$ bestehen $(\boldsymbol{S} .19$.$) , wobei$ 
9. Steiner, algeb. Curven, welche Mittelp. haben, u. Eigens. allgem. algeb. Curven. 79

sodann von diesen zwei Kegelschnitten der eine durch irgend 4 Paar Grundpuncte $q$ und $y_{1}$ und der andere durch die übrigen 4 Paare geht, und wobei die gegenseitigen 4 Schnitte von $J^{2}$ und $J_{1}^{2}$ zwei Paar Doppelpuncte $\mathfrak{p}_{2}$ und $\mathfrak{p}_{2}^{\prime}$ sind, so dafs also diese specielle Polare für zwei Polaren $\boldsymbol{J}_{2}^{4}$ zählt, und dafs die ihr entsprechende Ortscurve $\boldsymbol{P}_{2}^{x}$ den Pol $\boldsymbol{P}$ zum Doppelpunct haben mufs. Hierbei kann gefragt werden: $a$. Welches ist, in Bezug auf den gegebenen Curven-Büschel $\boldsymbol{B}\left(C^{5}\right)$, der Ort des Pols, für welchen die besondere Polare $\boldsymbol{J}_{0}^{4}$ aus $\boldsymbol{J}^{3}+\boldsymbol{S}_{2}$ besteht? (höchstens vom $50^{\text {sten }} \mathrm{Grad}$ ). $\boldsymbol{b}$. Welches ist der Ort des Pols, für welchen eine der 10 Polaren $\boldsymbol{J}_{2}^{4}$ aus $\boldsymbol{J}^{2}+\boldsymbol{J}_{1}^{2}$ besteht? oder welches ist der Ort der Doppelpuncte aller Ortscurven $\boldsymbol{S}\left(\boldsymbol{P}_{2}^{x}\right)$ ?

In Betreff der obigen allgemeinen Betrachtung sind, unter andern, folgende Aufgaben zu stellen.

1. Den Grad $x$ der Ortscurve $\boldsymbol{P}_{2}^{x}$ allgemein zu bestimmen, d.h. für jede Basis $C^{m}$.

2. Die Enveloppe der su einem gegebenen Curven-Büschel $\boldsymbol{B}\left(C^{m}\right)$ gehörigen Schaar Ortscurven $\boldsymbol{S}\left(\boldsymbol{P}_{2}^{x}\right)$ zu finden; und

3. Die Enveloppe der zu demselben Curven-Büschel gehörigen Schaar Ortscurven $\boldsymbol{S}\left(\boldsymbol{P}_{1}^{2 m-4}\right)=\boldsymbol{S}\left(\boldsymbol{D}^{m-1}\right)$ zu finden. Hierbei will ich bemerken, dafs diese Curvenschaar in der Hinsicht, dafs sie $(m-1)^{\text {ster }}$ Classe ist und durch jeden Punct $\boldsymbol{P}$ der Ebene je $2(\boldsymbol{m}-2)$ derselben gehen: auffallende Übereinstimmung mit einem Curven-Büschel gleicher Classe $\boldsymbol{B}\left(\boldsymbol{K}^{m-1}\right)$ und mit $(m-1)^{2}$ gemeinschaftlichen Tangenten hat, indem auch hier durch jeden Punct der Ebene je $2(m-2)$ Curven $K^{m-1}$ gehen; dagegen sind diese Curven $K^{m-1}$ vom $(m-1)(m-2)^{\text {ten }}$ Grad, während jene $D^{m-1}$ nur vom $2(m-2)^{\text {ten }}$ Grad, $=\boldsymbol{P}_{1}^{2 m-4}$, sind. Der $\boldsymbol{B}\left(\boldsymbol{K}^{m-1}\right)$ ist durch $\frac{1}{2} m(m+1)-2$ gegebene Tangenten bestimmt.

\section{\$. 22.}

I. Die innere Panpolare kann unter geeigneten Umständen auch in Theile zerfallen, wie aus Folgendem erhellen wird.

Befindet sich unter den Curven des gegebenen Büschels $\boldsymbol{B}\left(\boldsymbol{C}^{m}\right)$ insbesondere eine solche, etwa $C_{0}^{m}$, welche einen Mittelpunct $C_{0}$ hat, und wird dieser Mittelpunct als Pol angenommen: so zerfällt die innere Panpolare nothwendig in die Curve $C_{0}^{m}$ und in eine bestimmte Curve $J_{0}^{m-1}$, so dafs $J^{2 m-1}$ aus $C_{0}^{m}+J_{0}^{m-1}$ besteht, und $z$ war ist dabei die Curve $\boldsymbol{J}_{0}^{m-1}$ eine gemeinsame innere Polare aller gegebenen Curven $\boldsymbol{B}\left(\boldsymbol{C}^{m}\right)$, mit Ausnahme der Curve $\boldsymbol{C}_{0}^{m}$, mit welcher sie concentrisch ist. Ein solcher Pol $C_{0}(=P)$ bedingt aber 
80 2. Steiner, alyeb. Curven, welche Mittelp. haben, u. Eigens. allgem. algeb. Curven.

auch in Rücksicht der ihm entsprechenden äufsern Polaren, $\boldsymbol{B}\left(\boldsymbol{A}^{m-1}\right)$, eine besondere Eigenschaft, nämlich: Dafs alle diese Polaren parallele Asymptoten haben, und zwar parallel den Asymptoten jener gemeinsainen innern Polare $J_{0}^{m-1}$, weil sie mit dieser die Gerade $G_{\infty}$ in den nümlichen m-1 Puncten $a_{\infty}$ schneiden. Die $(m-1)(m-2)$ übrigen gemeinschaftlichen Puncte (Grundpuncte) $\boldsymbol{p}$ des Büschels $\boldsymbol{B}\left(\boldsymbol{A}^{m-1}\right)$ müssen daher in einer Curve $\boldsymbol{A}^{m-2}$ liegen, welche mit $\boldsymbol{G}_{\infty}$ zusammen eine Curve $\boldsymbol{A}_{0}^{m-1}$ dieses Büschels vertritt, und zwar ist dieselbe die äufsere Polare von jener besondern Curve $\boldsymbol{C}_{0}^{m}$. Befindet sich unter den gegebenen Curven $\boldsymbol{B}\left(\boldsymbol{C}^{m}\right)$ zugleich noch eine andere, etwa $\boldsymbol{C}_{(0)}^{m}$, welche einen Mittelpunct $\boldsymbol{C}_{00}$ hat, so kommen ihr und ihrem Mittelpuncte gleiche Eigenschaften zu. Bewegt sich sodann der Pol $\boldsymbol{P}$ in der durch beide Mittelpuncte gehenden Geraden $\boldsymbol{C}_{0} \boldsymbol{C}_{00}$, so entspricht ihm in jedem Moment ein Büschel äufsere Polaren $B\left(A^{m-1}\right)$ mit $(m-1)^{2}$ Grundpuncten $p:$ und der Ort aller dieser Puncte $p$ ist eine Curve $2(m-1)^{\text {sten }}$ Grads $A^{2 m-2}$, deren Asymptoten mit den Asymptoten der beiden gemeinsamen innern Polaren $J_{0}^{m-1}$ und $J_{(x)}^{m-1}$ parallel sind, oder welche mit diesen zwei Curven die Gerade $G_{\infty}$ in den nümlichen zweimal $m-1$ Puncten $a_{\infty}$ schneidet.

Durch Umkehrung ergeben sich folgende Sätze.

a. Giebt es in der Ebene zueier gegebenen Curven $m^{\text {ten }}$ Grads, $\boldsymbol{C}_{1}^{m}$ und $\boldsymbol{C}_{2}^{m}$, irgend einen solchen $\boldsymbol{P}_{\mathrm{ol}} \boldsymbol{C}_{0}(=\boldsymbol{P})$, dessen innere Polaren in Bezug auf dieselben in eine zusammenfallen, $J_{0}{ }^{m-1}$, so haben alle Curven des durch die zwei gegebenen bestimmten Büschels $\boldsymbol{B}\left(\boldsymbol{C}^{m}\right)$ für den gleichen Pol die nümliche innere Polare gemein, und so enthält dieser Büschel allemal eine solche besondere Curvé $C_{0}^{m}$, welche den Pol $C_{0}$ zum Mittel-' punct hat.

b. Hat eine gegebene Curve $m^{\text {ten }}$ Grads $C_{0}^{m}$ einen Mittelpunct $C_{0}$ und man nimmt in derselben $\frac{1}{2} m(m+3)-1$ Puncte $p$ beliebig an, so gehen durch diese Puncte unzählige Curven desselben Grads, $\boldsymbol{B}\left(C^{m}\right)$, welchen rücksichtlich jenes Mittelpuncts, als Pol, eine und dieselbe innere Polare $\mathbf{J}_{0}^{m-1}$ entspricht.

c. Sind in einer Ebene zwei Curven $m^{\text {ten }}$ Grads $C_{0}^{m}$ und $C_{00}^{m}$ gegeben, die Mittelpuncte $C_{0}$ und $C_{00}$ haben, so gehen durch ihre $m^{2}$ gemeinschaftlichen Puncte p unzïhlige andere Curven gleichen Grads, ein $\boldsymbol{B}\left(C^{m}\right)$, welche für jeden jener zwei Mittelpuncte die innere Polare $J_{0}^{m}{ }^{-1}$ und $J_{00}^{m-1}$ gemein haben, aber keine dieser Curven kann ebenfalls einen Mittelpunct haben; sind jedoch die gegebenen Curven concentrisch, so hat auch jede 
2. St einer, algeb. Curven, welche Mittelp. haben, u. Eigens. allgem. algeb. Curven. 81

dieser andern Curven einen Mittelpunct, und swar sind alle mit den gegebenen concentrisch. Nur für $m=2$ oder für den Kegelschnitt-Büschel $\boldsymbol{B}\left(\boldsymbol{C}^{2}\right)$ findet hierbei Abweichung statt.

II. In Betracht des allgemeinen Curven - Büschels $\boldsymbol{B}\left(\boldsymbol{C}^{m}\right)$ gehen durch jeden Pol $\boldsymbol{P}$ in Rücksicht jeder einzelnen Curve ein bestimmtes System von je $\frac{1}{2} m(m-1)$ Sehnen $\boldsymbol{S}$, die durch $\Sigma(\boldsymbol{S})$ bezeichnet werden mögen, so dafs also eben soviele solche Systeme statt finden, als der Büschel Curven enthält. Da nun die innere Panpolare desselben Pols vom $(2 m-1)^{\text {sten }}$ Grad ist, so fallen in jede durch $\boldsymbol{P}$ gezogene Gerade $\boldsymbol{G}$ je $m-1$ Sehnen $\boldsymbol{S}$, welche, im Allgemeinen, eben sovielen verschiedenen Curven oder Systemen $\boldsymbol{\Sigma}(\boldsymbol{S})$ angehören werden. Bezeichnet man irgend eine Curve des Büschels durch $\boldsymbol{C}_{1}^{m}$, ihre Sehnen durch $\Sigma\left(S_{1}\right)$ und jede der $\frac{1}{2} m(m-1)$ Geraden, in welchen die Sehnen liegen, durch $G_{1}$, so fallen in jede $G_{1}$ noch $m-2$ andere Sehnen $\boldsymbol{S}$, die eben so vielen andern Curven $C^{m}$ angehören, und es entsteht die Frage: $O b$ in Rücksicht aller $\frac{1}{2} m(m-1)$ Geraden $G_{1}$ die je $m-2$ andern Sehnen $S$ zu den nümlichen $m-2$ andern Curven $C^{m}$, oder ob dieselben im Ganzen zu $\frac{1}{2} m(m-1) \times(m-2)$ verschiedenen Curven $C^{m}$ gehören? Z. B. bei einem gegebenen $\boldsymbol{B}\left(\boldsymbol{C}^{3}\right)$ fällt in jede der 3 Geraden $\boldsymbol{G}_{1}$, in denen die 3 Sehnen $\boldsymbol{S}_{1}$ der Curve $\boldsymbol{C}_{1}^{3}$ liegen, noch je eine andere Sehne $\boldsymbol{S}^{\prime}$ und es ist die Frage, ob diese $3 \boldsymbol{S}^{\prime}$ einer und derselben, etwa $C_{2}^{3}$, oder ob sie drei verschiedenen andern Curven $\boldsymbol{C}^{3}$ angehören? Gehörten sie derselben Curve $\boldsymbol{C}_{2}^{3}$ an, so wären alle Curven des Büschels $\boldsymbol{B}\left(\boldsymbol{C}^{3}\right)$ einander paarweise zugeordnet. Es kann ferner gefragt werden: Welche Relation findet zwischen den $\frac{1}{2} \boldsymbol{m}(\boldsymbol{m}-1)$ Sehnen jedes Systems $\Sigma(\boldsymbol{S})$ statt? oder wenn eine derselben gegeben ist, wie sind die übrigen zu finden? Welche Relation findet zwischen verschiedenen Sehnen-Systemen statt?

Hat insbesondere eine der gegebenen Curven $\boldsymbol{B}\left(\boldsymbol{C}^{m}\right)$, etwa $\boldsymbol{C}_{0}^{m}$, einen Mittelpunct $\boldsymbol{C}_{0}$ und wird dieser als Pol $\boldsymbol{P}$ angenommen, wobei die Panpolare $\boldsymbol{J}^{2 m-1}=\boldsymbol{C}_{0}^{m}+\boldsymbol{J}_{0}^{m-1}$ wird, so liegen in jeder Geraden $\boldsymbol{G}$ je $\frac{1}{2} \boldsymbol{m}$ Sehnen $\boldsymbol{S}_{\mathbf{0}}$ der Curve $\boldsymbol{C}_{0}^{m}$, so wie je $\frac{1}{2}(m-1)$ Sehnen $\boldsymbol{S}$, welche einzeln andern Curven $C^{m}$ angehören; dabei sind in den Zahlen $\frac{1}{2} m$ und $\frac{1}{2}(m-1)$ nur die Ganzen zu zählen. Welche Resultate erhält man, wenn die eben aufgestellten Fragen auf diesen besondern Fáll angewendet werden? 
82 2. St ein er, algeb. Curven, welche Mittelp. haben, u. Eigens. allgem. algeb. Curven.

\$. 23.

Allgemeine Bemerkung.

Die Resultate der vorstehenden Untersuchung lassen sich durch Projection in andere, scheinbar allgemeinere, umwandeln; was bereits schon oben gelegentlich an einigen Stellen geschehen, an andern nur bemerkt worden ist. Auch kann, fast durchgängig, eine entgegenstehende Reihe von Sätzen und Eigenschaften nach dem Princip der Dualität der Raumgestalten gleicherweise entwickelt, oder durch Polarisation mittelst eines Hülfs-Kegelschnittes aus dem Obigen hergeleitet werden; welches alles zur Genüge bekannt ist. Eine eigentliche Weiterführung des Gegenstandes gedenke ich später mitzutheilen, wofern mein kränkelnder Zustand mir die zur Ausarbeitung nöthige Kraft und Anstrengung gestattet.

\section{Einiges über geradlinige Transversalen bei algebraischen Curven.}

\section{\$. 24.}

In den obigen Betrachtungen kommen bereits viele Sätze über solche Gerade vor, welche eine gegebene Curve unter irgend welchen bestimmten Bedingungen schneiden, und zwar wurde dabei, in den meisten Fällen, entweder der Ort der Geraden selbst, oder irgend eines in ihr fixirten Punctes, oder es wurden beide Örter zugleich berücksichtigt, so wie auch andere, davon abhängige Eigenschaften beobachtet. Einige der frühern Fälle sollen hier etwas allgemeiner behandelt und nebstdem noch andere analoge Beispiele hinzugefügt werden. Eine umfassendere Untersuchung über Transversalen bei algebraischen Curven, aus welcher nicht nur alle diese Beispiele, sondern auch ein grofser Theil der obigen Betrachtungen entnommen ist, behalte ich mir fôr eine andere Gelegenheit vor.

\section{\$. 25.}

Eine gegebene Curve $m^{\text {ten }}$ Grads $C^{m}$ wird von jeder in ihrer Ebene liegenden Geraden oder Transversale $\boldsymbol{S}$ in $m$ Puncten $a$ (reell oder imaginär) geschnitten. Der gemeinsame Schwerpunct solcher $m$ Schnitte (alle gleich schwer gedacht) heifse $\boldsymbol{A}$; so hat man, in Rücksicht dieses Schwerpunctes, zunächst folgende Sätze (wovon der erste I. a. allgemein bekannt ist):

I. a. ,Wird die Transversale $\mathbf{S}$ sich selbst parallel bewegt, so beschreibt ihr Schwerpunct A (d.h. der Schwerpunct ihrer veränderlichen 
2. St einer, algeb. Curven, welche Mittelp. haben, u. Eigens. allgem. algeb. Curven. 83

m Schnitte a) irgend eine bestimmte Gerade $\boldsymbol{D}$, nämlich den der Richtung von $\mathbf{S}$ conjugirten Durchmesser der Basis $C^{m}$."

b. „Giebt man der Transversale $\boldsymbol{S}$ nacheinander alle Richtungen, so entstehen alle Durchmesser $D$ der Basis, wozu insbesondere auch ihre $m$ Asymptoten $A_{s}$ gehören, und zwar als diejenigen eigenthümlichen Durchmesser, welche ihrer eigenen Richtung conjugirt sind; daher liegt der Schwerpunct $A$ jeder Transversale $\boldsymbol{S}$, welche einer $\boldsymbol{A}_{\text {s }}$ parallel ist, in dem unendlich entfernten Puncte $a_{\infty}$ der letstern; der Schwerpunct der Geraden $G_{\infty}$ aber, diese als Transversale angesehen, ist unbestimmt, liegt in jedem Durchmesser, also nach jeder Richtung. Alle Durchmesser insgesammt berühren eine bestimmte Curve $(m-1)^{\text {ster }}$ Classe $D^{m-1}$ und $2(\boldsymbol{m}-2)^{\text {ten }}$ Grads $\mathfrak{D}^{2 m-4}$, und zwar berührt jede $\boldsymbol{A}_{\text {s }}$ namentlich in demjenigen Puncte, etwa $a_{0}$, welcher der Schwerpunct ihrer m-1 Schnitte mit den m-1 übrigen Asymptoten ist; diese Curve hat, im Allgemeinen, $\frac{1}{2}(m-1)(m-2)$ Doppeltangenten, welche solche Durchmesser der Basis sind, $\boldsymbol{D}_{2}$, denen zwei verschiedene conjugirte Richtungen entsprechen." Demnach gehen durch jeden Punct $P$ der Ebene, im Allgemeinen, $m-1$ Durchmesser $\boldsymbol{D}$ der Basis, oder jeder Punct $\boldsymbol{P}$ ist der Schwerpunct $\boldsymbol{A}$ von $\dot{m}-1$ durch ihn gehenden Transversalen $S$, welche nämlich jenen Durchmessern beziehlich conjugirt sind; liegt der Punct $\boldsymbol{P}$ insbesondere in einer $A_{s}$, so ist diese selbst einer der $m-1$ Durchmesser, und so fällt die ihr conjugirte Transversale $\mathbf{S}$ auf sie, und da diese Transversale mit den übrigen, wie zuvor, ihren Schwerpunct in $\boldsymbol{P}$ haben mu/s, so kann also jeder Punct $\boldsymbol{P}$ in der Asymptote $\boldsymbol{A}_{s}$ als Schwerpunct einer auf ihr liegenden Transversule $\boldsymbol{S}$ angesehen werden; wird $\boldsymbol{P}$ in den im Unendlichen liegenden Punct $a_{\infty}$ der $\boldsymbol{A}_{s}$ versetst, so sind die übrigen $m-2$ Durchmesser $D$ alle mit $\boldsymbol{A}_{s}$ parallel, die ihnen conjugirten $m-2$ Transversalen fallen sümmtlich auf $\boldsymbol{G}_{\infty}$ und die der $\boldsymbol{A}_{s}$ conjugirte liegt auf dieser, wie zuvor; liegt endlich $P$ nach beliebiger Richtung in der Geraden $G_{\infty}$, so sind eben so alle $m-1$ Durchmesser nach dieser Richtung parallel und die ihnen conjuyirten Transversalen fallen alle auf $G_{\infty}$.

c. Da die Basis $C^{m}$ von der $m(m-1)^{\text {sten }}$ Classe ist, so hat sie mit der Curve $\boldsymbol{D}^{m-1}$ im Ganzen $\boldsymbol{m}(\boldsymbol{m}-1) \times(m-1)$ Tangenten gemein, d. h. ,Die Basis wird, im Allgemeinen, von $m(m-1)(m-1)$ ihrer Durchnesser berührt;" zu diesen besondern Durchmessern gehören namentlich die m Asymptoten $\boldsymbol{A}_{s}$, deren Berührungspuncte $\boldsymbol{a}_{\infty}$ auf $\boldsymbol{G}_{\infty}$ liegen; die $\boldsymbol{m}^{2}(\boldsymbol{m}-2)$ übrigen 
sollen durch $\boldsymbol{D}_{0}$ und ihre Berührungspuncte mit der Basis durch $\boldsymbol{d}_{0}$ bezeichnet werden.

II. a. ,Wird eine beliebige Transversale $S$ um irgend einen in ihr liegenden Pol P herumbewegt, so beschreibt ihr Schwerpunct A eine Curve $m^{\text {ten }}$ Grads, $A^{m}$, und $2(m-1)^{\text {ster }}$ Classe, welche den Pol zum $(m-1)$ fachen Punct und in demselben diejenigen $m-1$ Transversalen, von denen er der Schwerpunct ist (I. b.), zu Tangenten hat, und deren $m$ Asymptoten $\mathfrak{A}_{s}$ beziehlich den $m \boldsymbol{A}_{s}$ der Basis $C^{m}$ parallel sind, und zwor sind die beiden vollständigen mseit, $m \mathfrak{A}_{s}$ und $m \boldsymbol{A}_{s}$, ähnlich und ähnlichliegend, haben den Pol $\boldsymbol{P}$ zum Ähnlichkeitspunct und ihre homologen Dimensionen verhalten sich wie 1:m." Somit haben alle solche Curven $\boldsymbol{A}^{m}$, welchen Polen $\boldsymbol{P}$ sie entsprechen mögen, congruente Asymptotenm seit, $m \mathfrak{A}_{s}$; und denkt man sich, nebst der gegebenen Basis $C^{m}$, beliebige andere Curven $C_{1}^{m}, C_{2}^{m}, \ldots$, welche mit ihr die $m \boldsymbol{A}_{\text {s }}$ gemein haben, so mu/s jedem Pol $\boldsymbol{P}$, in Rücksicht aller dieser Curven, eine und dieselbe Curve $A^{m}$ entsprechen, und eben so haben dieselben alle Durchmesser $\boldsymbol{D}$ und deren Enveloppe $D^{m-1}$ gemein. Unter den Curven $C_{1}^{m}, \ldots$, welche mit $\boldsymbol{C}^{m}$ die $m$ Asymptoten $\boldsymbol{A}_{s}$ gemein haben, giebt es insbesondere eine solche, etwa $C_{p}^{m}$, welche den Pol $\boldsymbol{P}$ zum $(m-1)$ fachen Punct hat, und zuar hat dieselbe in diesem Puncte mit der Curve $A^{m}$ die genannten $m-1$ Tangenten gemein, so da/s ihre respectiven Zweige einander daselbst berühren, oder mit einem Worte: die Curven $C_{p}^{m}$ und $A^{m}$ sind ähnlich und ähnlichliegend, haben $\boldsymbol{P}$ zum $\ddot{A} h n l i c h k e i t s p u n c t$ und ihre entsprechenden Dimensionen verhalten sich, wie $m: 1$. - Denkt man sich den Pol $\boldsymbol{P}$ nach irgend einer Richtung ins Unendliche versetzt, so zerfällt die Curve $\boldsymbol{A}^{m}$ in den der Richtung conjugirten Durchmesser $\boldsymbol{D}^{-}$und in einen andern Theil, welcher ganz im Unendlichen liegt.

b. Da die Ortscurve $\boldsymbol{A}^{m}$ durch die im Unendlichen, in $\boldsymbol{G}_{\infty}$, liegenden $m$ Puncte $a_{\infty}$ der Basis $C^{m}$ geht (vermöge der Parallelität der Asymptoten), so müssen die übrigen $m(m-1)$ gemeinschaftlichen Puncte, etwa $A_{1}$, beider Curven in einer Curve $(\boldsymbol{m}-1)^{\text {sten }} \mathrm{Grads}, \boldsymbol{A}_{1}^{m-1}$, liegen. Oder: ,Durch jeden Pol P gehen je $m(m-1)$ solche besondere Transversalen $\mathbb{S}_{1}(=S)$, deren Schwerpuncte $A_{1}$ in der Basis selbst liegen, und zwar ihre Schnitte mit irgend einer Curve $(m-1)^{\text {sten }}$ Grads, $A_{1}^{m-1}$, sind." Liegt der Pol $P$ in der Basis selbst, so wird diese in demselben von der Curve $A_{1}^{m-1}$ (m-1)punctig berührt; die Berührung wird mpunctig, wenn eine jener 
2. Steiner, algeb. Curven, welche Mittelp. haben, u. Eigens. allgem. algeb. Curven. 85

m-1 Transversalen, welche $\boldsymbol{P}$ sum Schwerpunct haben, auf die Tangente der Basis in $\boldsymbol{P}$ fällt, was jedoch nur für eine bestimmte Zahl (nümlich für $\left.m(m-1)^{2}\right)$ Pole eintreten kann. Versetzt man den Pol $\boldsymbol{P}$ nach irgend einer Richtung ins Unendliche, so bleiben von den $m(m-1)$ Transversalen $\mathbf{S}_{1}$ nur noch $m$ wahrnehmbar; sie gehen nach derselben Richtung parallel und ihre $m$ Schwerpuncte $A_{1}$ liegen in dem der Richtung conjugirten Durchmesser $\boldsymbol{U}$ und sind dessen Schnitte mit der Basis; die $m(m-2)$ übrigen Transversalen $S_{1}$ fallen auf die Gerade $G_{\infty}$ und ihre Schwerpuncte liegen $z$ u je $m-2$ in den $m$ Puncten $a_{\infty}$ der Basis, so da/s also in diesem Falle die Curve $\boldsymbol{A}_{1}^{m-1}$ aus $\boldsymbol{D}+(m-2) \boldsymbol{G}_{\infty}$ besteht. - Bei dem besondern Falle, in welchem sich die vorgenannten beiden Curven $\boldsymbol{C}_{p}^{m}$ und $\boldsymbol{A}^{m}$ befinden (a.), liegen ihre $m(m-1)$ gemeinschaftlichen Puncte $\boldsymbol{A}_{1}$ sämmtlich im $\mathrm{Pol} \boldsymbol{P}$, und die Curve $\boldsymbol{A}_{1}^{m-1}$ zerfällt in ihre $m-1$ Berührungstangenten in demselben.

Wenn insbesondere die Curve $A^{m}$ die Basis $C^{m}$ in irgend einem Puncte berülst, so berührt auch die zugehörige Curve $A_{1}^{m-1}$ im nümlichen Puncte.

c. Bewegt sich der Pol $\boldsymbol{P}$ in irgend einer festen Geraden $\boldsymbol{G}$, so bilden die ihm, in Bezug auf die gegebene Basis $C^{m}$, in obigem Sinne (a.) entsprechenden Curven $\boldsymbol{A}^{m}$ eine solche Curven-Schaar $\boldsymbol{S}\left(\boldsymbol{A}^{m}\right)$, welche, aufser den $m$ Puncten $a_{\infty}$ der Basis, auch noch den Schwerpunct, etwa $A_{g}$, der Geraden $\boldsymbol{G}$ gemein haben, und welche nebstdem die ganze Ebene dergestalt durchziehen, dafs durch jeden beliebigen Punct $\mathfrak{S}$, im Allgemeinen, je $m-1$ derselben gehen, und dafs die Basis in jedem ihrer $m$ Puncte $a_{\infty}$ von je einer derselben zweipunctiğ, in ihren $m$ Schnitten mit der Geraden $\boldsymbol{G}$ aber von je einer derselben $(m-1)$ punctig und au/serdem noch von $m\left(m^{2}-3\right)$ derselben in je einem andern Puncte berührt uird. „Zudem haben die $\mathbf{S}\left(\boldsymbol{A}^{m}\right)$ die obige Curve $D^{m-1}$ ( I. b.) sur gemeinsamen Enveloppe, und zwar wird diese von jeder Curve $A^{m}$, im Allgemeinen, in $2 m-3$ verschiedenen Puncten berührt; insbesondere giebt es unter denselben $4(m-2)$ solclue, welche die Curve $D^{m-1}$ in irgend einem Puncte vierpunctig (also nebstdem nur noch in $2 m-5$ Puncten) berühren." „Ferner ist diese Schaar Curven $\boldsymbol{S}\left(A^{m}\right)$ so beschaffen, da/s irgend eine gegebene Curve $q^{\text {ten }}$ Grads von $q(q+1)(m-1)$ derselben berührt wird;" also wird insbesondere jede gegebene Gerade von je $2(m-1)$ derselben berührt. *)

*) Bewegt sich der Pol P, statt in der Geraden G, in irgend einer Curve $n^{\text {ten }}$ Grads $G^{n}$, so hat die ihm entsprechende Curven-Schaur $S\left(A^{m}\right)$ folgende Eigen- 
86 2. Stein er, algeb. Curven, welche Mittelp. haben, u. Eigens. allgem. algeb. Curven.

Beachtet man, während der Pol $\boldsymbol{P}$ sich in der Geraden $\boldsymbol{G}$ bewegt, die je $m-1$ Transversalen, von welchen er der Schwerpunct ist (I. b.), so sind dieselben zusammen alle Transversalen, deren Schwerpuncte in $\boldsymbol{G}$ liegen; wird jede derselben durch $\boldsymbol{S}_{g}$ bezeichnet, so hat man den Satz:

„Der Ort aller Transversalen $\boldsymbol{S}_{g}$, deren Schwerpuncte $A$ in irgend einer gegebenen Geraden $G$ liegen, ist eine Curve $m^{\text {ter }}$ Classe $\mathbb{S}_{g}^{m}$ und $2(m-1)^{\text {sten }}$ Grads, welche die Gerade $G_{\infty}$ zur $(m-1)$ fachen Tangente hat, und welche namentlich die Asymptoten $\boldsymbol{A}_{\text {s }}$ der Basis, so wie auch die Gerade $G$, und zwar diese in ihrem Schwerpuncte $A_{g}$ berührt."

Dieser und der obige erste Satz (a.) sind gewissermafsen einander entgegenstehend. Durch Hülfe derselben folgen leicht alle nachstehenden Sätze.

III. a. ,Soll die durch die feste Basis $\boldsymbol{C}^{m}$ gezogene Transversale $\boldsymbol{S}$ irgend eine andere gegebene Curve $n^{\text {ter }}$ Classe $\boldsymbol{K}^{n}$ berühren, so ist der Ort ihres Schwerpuncts $A$ eine Curve mn $n^{\text {ten }}$ Grads, $A^{m n}$, welche die in Puncte $a_{\infty}$ der Basis zu nfachen Puncten und daher mit jeder $\boldsymbol{A}_{\text {s }}$ der Basis je $n$ parallele Asymptoten $\mathfrak{A}_{s}$ hat, welche den, nach gleicher Richtung gehenden $n$ Tangenten $T$ der Basis in der Art entsprechen, da/s die Abstände je zueier zusammengehörigen $\mathfrak{A}_{s}$ und $\boldsymbol{T}$ von $\boldsymbol{A}_{s}$ sich verhallen wie $m-1: m . "$

b. ,Soll der Schwerpunct $A$ der Transversale $S$ in irgend einer gegebenen Curve $n^{\text {ten }}$ Grads $\boldsymbol{G}^{\text {n }}$ liegen, so ist ihr Ort eine Curve m $n^{\text {ter }}$ Classe $\boldsymbol{S}^{m n}$ und $\boldsymbol{n}(\boldsymbol{n}-1)(m-1)+2 n(m-1)=(m-1) n(n+1)^{\text {ten }}$ Grads, welche die $m$ Asymptoten $\boldsymbol{A}_{s}$ der Basis zu nfachen und die Gerade $G_{\infty}$ zur.

schaften: $1^{\circ}$. Sie haben die $m$ Puncte $a_{\infty}$ der Basis gemein. 20. Durch jeden Punct P der Ebene gehen, im Allyemeinen, $n(m-1)$ Curven $A^{m}$. $3^{\circ}$. Die Basis $C^{\prime n}$ wird in jedem der $m$ Puncte $a_{\infty}$ von $n$ derselben einfach, in ihren mn Schnitten mit der Curve $G^{n}$ von je einer derselben $(m-1)$ punctiy und aufserdem von $n m\left(m^{2}-3\right)$ derselben in andern bestimmten Puncten einfach berührt. 4'. Irgend eine gegebene Curve $q^{\text {ten }}$ Grads $Q^{q}$ wird, im Allgemeinen, von $n q(q+1)(m-1)$ Curven $A^{m}$ berïhrt. $5^{\circ}$. Die Enveloppe der $S\left(A^{m}\right)$ besteht im Ganzen: $\left.\alpha\right)$ aus den m Puncten $a_{\infty}$; $\beta)$ aus der Curve $D^{m-1}$ und suar wird diese, wie oben, von jeder $A^{m}$ in $2 m-3$ Puncten berührt, insbesondere giebt es $4 n(m-2)$ solche Curven $A^{m}$, welche dieselbe in irgend einem Puncte vierpunctiy berïhren; $\gamma)$ aus der $(m-1)(m-2)$ fuchen gegebenen Curve $G^{n}$; und endlich $\delta$ ) aus einer bestimmten Curve mn ${ }^{\text {ten }}$ Grads, die jedoch von jeder Curve $A^{m}$, im Allyemeinen, in nur einem Puncte berührt wird.

Beweyt sich der Pol $\boldsymbol{P}$ in der Basis $C^{m}$ selbst, so wird diese (abgesehen davon, dafs sie im jedesmaligen Pol von den zugehörigen Curven $A^{m}$ und $A^{m-1}(b$.) schon $(m-1)$ punctig berührt wird, aufserdem) von $m\left(m^{3}-m^{2}-m-1\right)$ Curven $A^{m}$ (und zugehörigen $\left.A_{1}^{m-1}\right)$ berïhrt, und zwar wird sie von $m(m-1)^{2}$ derselben im jedesmaligen Pol sclbst (also mpunctig), dagegen von $m(m-2)\left(m^{2}+1\right)$ derselben in andern bestimmten Puncten berïhrt. 
2. Steiner, algeb. Curven, welche Mittelp. haben, u. Eigens. allgem. algeb.Curven. 87

$n(m-1)$ fuchen Tangente hat; ihre $n(m-1)$ Berührungspuncte mit der letstern sind durch die conjugirten Richtungen derjenigen $n$ mal $m-1$ Durchmesser $D$ der Basis bestimmt, welche beziehlich den $n$ Asymptoten der gegebenen Curve $G^{n}$ parallel sind; ihre $n(n-1)(n-1)$ geradlinigen Asymptolen $\mathfrak{A}_{s}$ aber haben die conjuyirten Richtungen derjenigen Durchmesser der Basis, welche die Curve $G^{n}$ berühren, und gehen, beziehlich durch deren Beruhrungspuncte, so da/s sie dadurch vollkommen bestimnt sind."

IV. „Der Ort derjenigen Transversale $S_{1}$, deren Schwerpunct $A_{1}$ in der Basis $C^{m}$ selbst liegt (II. b.), also mit einem ihrer $m$ Schnitte a, der $a_{1}$ heifsen soll, zusammenfällt, ist eine Curve $m(m-1)^{\text {ster }}$ Classe $S_{1}^{m(n-1)}$ und $m\left(m^{2}-3\right)^{\text {ten }}$ Grads, welche die Gerade $G_{\infty}$ zur $m(m-2)$ fachen Tangente und mit der Basis deren m Asymptoten $A_{s}$ gemein, jedoch dieselben zugleich su (m-1)fachen Tangenten hat, und welche nebstdem die Busis in $m^{2}(m-2)$ bestimmten andern Puncten berührt." Ferner: „Die werden durch die conjugirten Richtungen derjenigen Durchmesser der Basis angezeigt, welche, zu je in-2, mit ihren m Asymptoten $A_{\text {s }}$ parallel sind (I. b.); die $m(m-1)^{2}$ geradlinigen Asymploten der erstern (worunter jene $m A_{s}$ mit inbegriffen) gehen durch diejenigen Puncte $d_{0}$ der Basis, in welchen diese von einzelnen ihrer Durchmesser $D_{0}$ berührt wird (I. c.), und zwar hat jede Asymptote die dem jedesmaligen Durchmesser conjugirte Richtung, so dafs sie bestimmt ist.

Die Curve $\boldsymbol{S}_{1}^{m(m-1)}$ hat mit der Basis $\boldsymbol{C}^{m}$ im Ganzen

$$
m(m-1) \times m(m-1)
$$

Tangenten gemein; daraus könnte man schliefsen, dafs die Basis, eben soviele solche Tangenten, etwa $\boldsymbol{S}_{1}^{0}$, habe, deren Schwerpuncte $\boldsymbol{A}_{1}$ in ihr selbst liegen: allein es verhält sich nicht genau so. Sondert man zunächst die $m$ Asymptoten $\boldsymbol{A}_{s}$ der Basis, wovon jede für $\boldsymbol{m}$, also alle für $m^{2}$ gemeinschaftliche Tangenten zählen, ab, so bleiben noch

$$
(m-2) m^{3}
$$

solche gemeinschaftliche Tangenten $\boldsymbol{S}_{1}^{0}$ übrig, deren Berührungspuncte, etwa $\boldsymbol{a}_{2}$, mit der Basis nicht im Unendlichen liegen, und in Rücksicht dieser entsteht nun die Frage: Bei wievielen derselben fällt der Schwerpunct $A_{1}$ mit dem Berührungspuncte $a_{2}$, und bei wievielen fällt er mit einem der $m-2$ 
88 2. Steiner, algeb. Curven, welche Mittelp. haben, u. Eigens. allgem. algeb. Curven.

Schnitte $a$, mit $a_{1}$, zusammen? Da im Berührungspuncte $a_{2}$ zwei der $m$ Schnitte a vereinigt sind, so habe ich die Wahrscheinlichkeit beider Fälle nach dem Verhältnifs von

$$
2: m-2
$$

angenommen, $\left.{ }^{*}\right)$ woraus sich ergiebt, da/s von den $(m-2) m^{3}$ Tangenten $\boldsymbol{S}_{1}^{\prime \prime}$ der Schwerpunct $A_{1}$

(1.) $2(m-2) m^{2} m a l$ in $a_{2}$; und (2.) $(m-2)^{2} m^{2}$ mal in einem $a_{1}$ liegen mu/s. Wenn aber der Schwerpunct $A_{1}$ im Berührungspunct $a_{2}$ liegt, so $m u / s$ die Curve $\boldsymbol{S}^{m(m-1)}$ daselbst nothwendig die $\boldsymbol{S}_{1}^{0}$ und somit auch die Basis $C^{m}$ berühren, so dafs also $\boldsymbol{S}_{1}^{\prime)}$ in diesem Falle (als BerührungsTangente) für zwei gemeinschafıliche Tangenten zählt, im Sinne von \$.17. eine $\boldsymbol{S}_{2}^{0}$ ist, und folglich der Schwerpunct $\boldsymbol{A}_{1}$ nur halb so oft, als eben angegeben worden (1.), also nur

$$
\text { (1*) } \quad(m-2) m^{2} \text { mal in } a_{2}
$$

fällt. Danach reduciren sich die $(m-2) m^{3}$ Tangenten $S_{2}^{0}$ auf

$$
(m-2)(m-1) m^{2}
$$

und mit diesen verhält es sich so:

„Die gegebene Basis $C^{m}$ hat, im Allgemeinen, au/ser den Asymptoten :

a) $(m-2) m^{2}$ solche Tangenten, deren Schwerpunct $A_{1}$ mit dem Berührungspunct $a_{2}$ zusammenfällt; und

b) $(m-2)^{2} m^{2}$ solche Tangenten, deren Schwerpunct $A_{1}$ in einem ihrer $m-2$ Schnitte $a$, in $a_{1}$, liegt." **)

Übrigens verhält es sich mit den $m$ Asymptoten $\boldsymbol{A}_{s}$ eben so. Da jede $\boldsymbol{A}_{s}$ für $m$ gemeinschaftliche Tangenten zählt, so müssen auch $m$ Schwerpuncte $\boldsymbol{A}_{1}$ in ihr und zugleich in der Basis $C^{m}$ liegen; und zwar vertheilen sich dieselben nach dem Verhältnifs von $2: m-2$, nämlich 2 sind im Berührungspuncte $a_{2}\left(=a_{\infty}\right)$ vereinigt, woselbst sich zugleich die beiden Curven berühren, und die $m-2$ andern fallen in die $m-2$ Schnitte $a$ von $\boldsymbol{A}_{s}$ und $\boldsymbol{C}^{m}$.

*) Einen strengen Beweis für die Richtigkeit oder Unrichtigkeit dieser Annahme überlasse ich Andern. Für $m=3$ und $m=4$, d. h. für die Basen $C^{3}$ und $C^{4}$, stimmt die Annahme mit der Wahrheit überein.

**) Wahrscheinlich können durch die $m^{2}(m-2)$ Puncte $a_{2}$ Curven $m(m-2)^{\text {ten }}$ Grads gehen; und alsdann gehen auch durch die $(m-2)^{2} m^{2}$ Puncte $a_{1}$ Curven $(m-2)^{2} m^{\text {ten }}$ Grads. Für die Basen $3^{\text {ten }}$ und $4^{\text {ten }}$ Grads ist es der Fall. 
2. St einer, algeb. Curven, welche Mittelp. haben, u. Eigens. allgem. alyeb. Curven. 89

Somit findet für die gesammten $m(m-1) \cdot m(m-1)$ gemeinschaftlichen Tangenten $\mathbf{S}_{2}^{0}$ dieselbe Reduction und Vertheilung statl; nämlich sie reduciren sich auf

$$
\boldsymbol{m}(\boldsymbol{m}-1)^{3}
$$

und von diesen liegt der Schwerpunct $A_{1}$

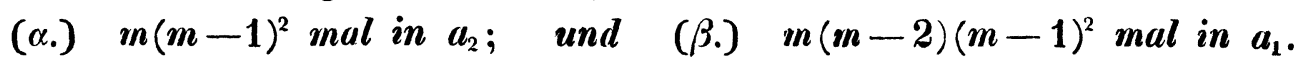

Hierbei, und mehr noch oben (I. b.), stellt sich für die Asymptote $\boldsymbol{A}_{s}$ das Eigenthümliche heraus, dafs ihr Schwerpunct, d. h. der Schwerpunct ihrer m gemeinschaftlichen Puncte a mit der Basis $C^{m}$, unbestimmt ist, in ihr liegen kann, wo man will; wogegen bei jeder mit $\boldsymbol{A}_{s}$ parallelen Transversale $\boldsymbol{S}$ der Schwerpunct bestimmt, nämlich im Unendlichen, in $a_{\infty}$ liegt. Dies erklärt sich einfach aus dem Umstande, dafs man sich bei $\boldsymbol{A}_{s}$ die zwei in ihrem Berührungspuncte $a_{2}$ vereinigten Puncte, etwa $a_{\infty}$ und $a_{\infty}^{\prime}$, nach entgegengesetzten Richtungen im Unendlichen denken kann, wobei sodann jeder beliebige Punct in $\boldsymbol{A}_{s}$ als in der Mitte zwischen ihnen liegend, also als ihr Schwerpunct anzusehen ist, was somit auch den Schwerpunct aller $m$ Puncte a unbestimmt macht. Eben so ist der Schwerpunct der Asymptote $\boldsymbol{A}_{s}$ bestimmt, oder unbestimmt, wenn sie die Basis im Punctc $a_{\infty}$ beziehlich dreipunctig oder vierpunctig berührt.

V. Der Ort der Schwerpuncte $\boldsymbol{A}_{0}$ aller Tungenten $\boldsymbol{S}_{0}$ der Basis $\boldsymbol{C}^{m}$, dieselben als Transversulen $\mathbf{S}$ angesehen, ist eine Curve $m\left(m^{2}-m-1\right)^{\text {sten }}$ Grads, $A_{0}^{m\left(m^{2}-m-1\right)}$, welche die $m$ Puncte $a_{\infty}$ der Basis $2 u\left(m^{2}-m-1\right)$ fachen Puncten hat, und zuar init dem einen Zweige daselbst die Basis berührt, dagegen mit den $m^{2}-m-2$ übrigen schneidet, und welche ferner die Basis in den nümlichen vorgenannten (m-2) $\mathrm{m}^{2}$ Puncten $a_{2}$ berührt und in den $(m-2)^{2} m^{2}$ Puncten $a_{1}$ schneidet (IV.). Danach hat die Ortscurve mit der Basis deren $m$ Asymptoten $\boldsymbol{A}_{s}$ gemein, aber mit jeder $\boldsymbol{A}_{s}$ noch $m^{2}-m-2$ andere Asymptoten $\mathfrak{A}_{s}$ parallel, welche den mit derselben $\boldsymbol{A}_{s}$ parallelen $m^{2}-m-2$ Tangenten $T$ der Basis so entsprechen, da/s die Abstände der zusummengehörigen $\mathfrak{A}_{s}$ und $\boldsymbol{T}$ von $\boldsymbol{A}_{s}$ sich verhalten wie $m-1: m . "$

VI. „Der Ort der Schwerpuncte $\boldsymbol{A}_{d}$ aller Durchmesser $D$ der. gegebenen Busis $\boldsymbol{C}^{m}$, dieselben als $\mathbf{S}$ angesehen, ist (au/ser den Asymptoten der Basis) eine Curve $m(m-2)^{t e n}$ Grads, $A_{d}^{m(m-2)}$, welche die $m$ Puncte $a_{\infty}$ der Basis zu (m-2)fachen Puncten und daher mit jeder $\boldsymbol{A}_{s}$ der letztern je $m-2$ parallele Asymptoten $\mathfrak{A}_{s}$ hat, die den mit derselben $\boldsymbol{A}_{s}$ parallelen Crelle's Journal f. d. M. Bd. XLVII. Heft 1. 
90 2. Steiner, algeb. Curven, welche Mittelp. habë, u. Eigens. allgem. algeb. Curven.

m-2 Durchmessern $D$ (I. b.) in der Art entsprechen, da/s die Abstände der zusammengehörigen $\mathfrak{A}_{s}$ und $\boldsymbol{D}$ von $\boldsymbol{A}_{s}$ sich verhalten, wie m $-1: m$; und welche ferner die Enveloppe aller Durchmesser, die Curve $D^{m-1}$ in denselben Puncten $a_{0}$ berührt, in welchen diese von den m Asymptoten $A_{s}$ der Basis berührt wird (I. b.)." Die m(m-2) $\times m$ gemeinschaftlichen Puncte der Curven $\boldsymbol{A}_{d}^{m(m-2)}$ und $\boldsymbol{C}^{m}$ sind die Schwerpuncte $\boldsymbol{A}_{d}$ eben so vieler Durchmesser der letztern; und da $m(m-2)$ derselben in die $m$ Puncte $a_{\infty}$ fallen, so zeigen die $m(m-1)(m-2)$ übrigen die Zahl derjenigen Durchmesser an, deren Schwerpuncte in der Basis, aber nicht im Unendlichen liegen. Dasselbe Resultat ergiebt sich auch, wenn man die $m(m-1)^{2}$ gemeinschaftlichen Tangenten der Curven $\boldsymbol{S}_{1}^{m(m-1)}$ (IV.) und $\boldsymbol{D}^{m-1}$ berücksichtigt; denn werden von diesen die (m-1)fach gezählten Asymptoten $\boldsymbol{A}_{s}$ weggelassen, so sind die $m(m-1)(m-2)$ übrigen gerade die genannten Durchmesser. Also:

„Die gegebene Basis $C^{m}$ hat, im Allgemeinen, $m(m-1)(m-2)$ solche Durchmesser, deren Schwerpuncte in ihr selbst, aber nicht im Unendlichen liegen, und durch diese Sihwerpuncte können Curven $(m-1)(m-2)^{\text {ten }}$ Grads gehen."

In Betracht der Durchmesser $D$ und ihrer Enveloppe $D^{m-1}$ ist noch der folgende Satz hinzuzufügen :

,Wird durch denjenigen Punct $a_{11}$, in welchem jeder Durchmesser $D$ der Basis $C^{m}$ die Enveloppe $D^{m-1}$ berührt, die dem Durchmesser conjugirte Transversale $\mathfrak{S}(=S)$ gezogen, so ist ihr Ort eine Curve $(2 m-3)^{\text {ter }}$ Classe, $\mathfrak{S}^{2 m-3}$, und $4(m-2)^{t e n}$ Grads, welche die Gerade $\boldsymbol{G}_{\infty}$ zur $2(m-2)$ fachen T'angente hat und namentlich auch die m Asymptoten der Basis berührt."

VII. Werden die vorstehenden Sätze auf die einfachsten Basen, $C^{3}$ und $C^{4}$, bezogen, so ergeben sich noch viele Folgerungen aus denselben; wie z. B. die nachstehenden.

a. Für die Basis $C^{3}$ sind die meisten Sätze bereits schon oben (\$. 15.) unter anderem Gesichtspuncte betrachtet worden; hier soll nur noch Einiges bemerkt werden. Nach (IV.) soll die Ortscurve $S_{1}^{6}$ die Basis $C^{3}$ in $(3-2) 3^{2}=9$ Puncten $a_{2}$ berühren, welche zugleich die Schwerpuncte $\boldsymbol{A}_{1}$ der zugehörigen Tangenten $S_{1}^{0}$ sind; und ferner soll von $(3-2)^{2} \cdot 3^{2}=9$ andern Tangenten $\boldsymbol{S}_{1}^{0}$ der Schwerpunct $\boldsymbol{A}_{1}$ zugleich im (einzigen) Schnittpuncte $\boldsymbol{a}_{1}$ derselben liegen. Diese 2 mal 9 Tangenten $S_{1}^{0}$ reduciren sich aber auf die 9 Wendetangenten $\mathfrak{W}$ der $C^{3}$, so dafs in jedem Wendepunct $\mathfrak{w}$ ein $a_{2}$ und ein $a_{1} \mathrm{zu}-$ gleich liegen (vergl. \$. 15. II. 2.). - Nach (V.) ist der Ort der Schwer- 
2. St ein er, algeb. Curven, welche Mittelp. haben, u. Eigens. allgem. algeb. Curven. 91

puncte $\boldsymbol{A}_{0}$ aller Tangenten $\boldsymbol{S}_{0}$ eine Curve $15^{\text {ten }}$ Grads $\boldsymbol{A}_{0}^{15}$, welche die drei Puncte $a_{\infty}$ der Basis $C^{\mathbf{3}}$ zu fünffachen Puncten hat und daselbst mit je einem Zweige die Basis berührt, so dafs sie mit dieser daselbst 18 Puncte gemein hat und sie nebstdem in ihren $9 \mathfrak{w}$ dreipunctig berührt, also mit ihr die $9 \mathfrak{B}$ gemein hat. Der Schwerpunct $\boldsymbol{A}_{0}$ jeder Tangente $\boldsymbol{S}_{0}$ liegt im ersten DrittelsPunct vom Berührungspuncte aus. Die Mitte jeder Tangente $\boldsymbol{S}_{1}$ beifse $\boldsymbol{M}$. Der Ort aller $M$ ist ebenfalls eine Curve $15^{\text {ten }}$ Grads $M^{15}$, welche die 3 Puncte $a_{\infty}$ zu fünffuchen Puncten hat, mit dem einen Zweige daselbst die $C^{3}$ beruhrt und mit dieser nebstdem die $9 \mathfrak{w}$ und zugehörigen $9 \mathfrak{W}$ gemein hat. Daher folgt: In der Ebene einer Curve $3^{\text {ten }}$ Grads $C^{3}$ giebt es, im Allyemeinen, 120 solche Puncte P Cau/ser den $3 a_{\infty}$ und $9 \mathfrak{w}$ ), wovon jeder $A_{0}$ und $M$ sugleich, d. h. der Drittelsoder Schwerpunct einer und die Mitte einer andern Tangente zugleich ist. - Nach (VI.) ist der Ort der Schwerpuncte $\boldsymbol{A}_{d}$ aller Durchmesser $\boldsymbol{D}$ eine Curve $3^{\text {ten }}$ Grads $\boldsymbol{A}_{d}^{3}$, welche mit $\boldsymbol{C}^{3}$ parallele Asymptoten hat; daher sind die Asymptoten-Dreiecke, $3 \mathfrak{A}_{s}$ und $3 \boldsymbol{A}_{s}$, beider Curven ähnlich und ähnlichliegend, und zwar haben sie den Schwerpunct gemein, somit zugleich zum Ähnlichkeitspunct, und zudern verhalten sich ihre entsprechenden Seilen, wie 1:3; und ferner berührt die Curve $A_{d}^{3}$ die Seiten des Asymptoten-Dreiecks der Basis in ihren Mitten $a_{0}$ und hat ihre eigenen Asymptoten zugleich zu Wendetungenten. U. s. w.

b. Bei der Basis $C^{4}$ kann, unter andern, Folgendes hervorgehoben werden. Nach (IV.) ist der Ort aller $\boldsymbol{S}_{1}$, deren Schwerpuncte $\boldsymbol{A}_{1}$ in $\boldsymbol{C}^{4}$ selbst liegen, eine Curve $12^{\text {ter }}$ Classe $S_{1}^{12}$ und $52^{\text {sten }}$ Grads, welche mit der Basis, aufser deren Asymptoten, $(4-2) 4^{3}=128$ Tangenten $\boldsymbol{S}_{1}^{0}$ gemein hat, aber von denen 32 Paare zusammenfallen, nur 32 Tangenten $\boldsymbol{S}_{2}^{\prime \prime}$ bilden, bei welchen der Schwerpunct $\boldsymbol{A}_{1}$ im Berührungspuncte $a_{2}$ liegl, und über welche das Weitere bereits oben $\left(\$ .17\right.$.) steht; wogegen bei den $(4-2)^{2} \cdot 4^{2}=64$ übrigen $S_{1}^{0}$ der Schwerpunct $A_{1}$ sich in einem der zwei Schnitte $a$, oder $a_{1}$, befindet. Bezeichnet man den Berührungspunct jeder der letztern Tangenten durch $\alpha$ und die zwei Schnitte durch $\beta$ und $\gamma$, und nimmt an, der Schwerpunct $\boldsymbol{A}_{1}$ liege in $\beta$ : so mufs $\beta$ zwischen $\alpha$ und $\gamma$ liegen, und zwar mufs die Strecke $\beta \gamma=2 \beta \alpha$ sein. Also: Eine beliebige Curve $4^{\text {ten }}$ Grads $C^{4}$ hat, im Allgemeinen, 64 solche Tangenten, bei denen die beiden Schnitte $\beta$ und $\gamma$ auf gleicher Seite vom Berührungspuncte a liegen, und wobei der eine Schnitt gerade dreimal so weit vom Berührungspunct abliegt, 
als der andere, $\alpha \gamma=3 \alpha \beta$." Durch die 64 Puncte $a_{1}$ (oder $\beta$ ) können Curven 16 $^{\text {ten }}$ Grads gehen. - Die Schwerpuncte $\boldsymbol{A}_{0}$ aller Tangenten $\boldsymbol{S}_{0}$ der $C^{4}$ liegen in einer Curve $44^{\text {sten }}$ Grads (V.). Der Ort der Schwerpuncte $\boldsymbol{A}_{d}$ aller Durchmesser $D$ ist eine Curve $8^{\text {ten }}$ Grads $\boldsymbol{A}_{d}^{8}$, welche die 4 Puncte $a_{\infty}$ und die drei Schnitte der drei Paar conjugirten Durchmesser der Basis ( $\$$. 17.) zu Doppelpuncten hat. Es giebt (aufser den $4 \boldsymbol{A}_{s}$ ) 24 solche Durchmesser $D$, deren Schwerpuncte $\boldsymbol{A}_{d}$ in $\boldsymbol{C}^{4}$ selbst liegen, und durch die $24 \boldsymbol{A}_{d}$ können Curven $6^{\text {ten }}$ Grads gehen (VI.).

Bemerkung., Durch Projection erhalten die vorstehenden Sätze ein allgemeineres Ansehen; nämlich an die Stelle des betrachteten Schwerpunctes tritt ein ,mittlerer harmonischer Punct," welcher übrigens auf die Art zu bestimmen ist, wie bereits Poncelet in seiner interessanten Abhandlung ,sur les centres de moyennes larmoniques" (Bd. 3. S.213 d. Journ.) gezeigt hat. \$. 26.

I. Die $m$ Schnitte $a, b, c, d, \ldots$ der Basis $C^{m}$ und irgend einer Transversalen $S$ begrenzen in der letztern $\frac{1}{2} m(m-1)$ Strecken $a b, a c, a d, \ldots$, $b c, b d, \ldots, c d, \ldots$; die Mitte jeder Strecke heifse $\boldsymbol{Q}$. Jede Strecke ist rücksichtlich ihrer Mitte eine einfache Sehne, etwa $s$ (stalt $\$$ ) (\$. 13.), und somit liegen in jeder Transversale $\boldsymbol{S}$, im Allgemeinen, $\frac{1}{2} m(m-1)$ einfache Sehnen $s$, und eben soviele Mitten $\boldsymbol{Q}$. Wenn insbesondere $\boldsymbol{S}$ die Basis berührt, so liegt eine Mitte $\boldsymbol{Q}$ im Berührungspunct, etwa $(a b)$, und $m-2$ Paare fallen zusammen. Ist ferner insbesondere $\boldsymbol{S}$ einer Asymptote $\boldsymbol{A}_{s}$ der Basis parallel, so hat man sich $m-1$ Puncte $Q$ als in $a_{\infty}$ liegend zu denken; und fällt $\boldsymbol{S}$ auf $\boldsymbol{A}_{s}$, so liegen $2(m-2)$ Puncte $\boldsymbol{Q}$ in $\boldsymbol{a}_{s}$, ein anderer Punct $\boldsymbol{Q}$ aber, nämlich die Mitte der im Berührungspuncte $a_{\infty}$ vereinigten zwei Puncte $a$ und $b$, bleibt hierbei unbestimmt, er kann jeder beliebige Punct in $\boldsymbol{A}_{s}$ sein (vergl. \$. 25. IV.); die noch übrigen $\frac{1}{2}(m-2)(m-3)$ Puncte $\boldsymbol{Q}$ sind bestimmt, wie zuvor. Wird $\boldsymbol{S}$ ins Unendliche versetzt, soll $\boldsymbol{S}=\boldsymbol{G}_{\infty}$ sein, so sind die Puncte $\boldsymbol{Q}$ unbestimmt, weil die Richtung von $\boldsymbol{G}_{\infty}$ unbestimmt ist; sobald aber die Richtung von $\boldsymbol{G}_{\infty}$ festgestellt wird, so sind auch alle $\frac{1}{2} m(m-1)$ Puncte $\boldsymbol{Q}$ bestimmt, nämlich durch Hülfe $\operatorname{der} m$ Asymptoten $\boldsymbol{A}_{s}$ der Basis. Diese Unbestimmtheit der Richtung von $\boldsymbol{G}_{\infty}$ bewirkt, dafs jeder nach irgend einer gegebenen Richtung in $\boldsymbol{G}_{\infty}$ liegende Punct $\boldsymbol{Q}_{\infty}$ nach Belieben als die Mitte von jeder durch die Puncte $a_{\infty}, b_{\infty}, c_{\infty}, d_{\infty}, \ldots$ begrenzten $\frac{1}{2} m(m-1)$ Strecken angesehen werden kann. - Mit Bezug auf alle diese Umstände hat man folgende Sätze. 
II. a. „Wird die beliebige Transversale $\mathbf{S}$ sich selbst parallel bewegt, so beschreiben ihre $\frac{1}{2} m(m-1)$ Mitten $Q$ insgesammt eine Curve $\frac{1}{2} m(m-1)^{s t e n}$ Grads, $Q^{\frac{1}{m}(m-1)}$, und $m(m-1)(m-2)^{\text {ter }}$ Classe, welche $\frac{1}{8} m(m-1)(m-2)(m-3)$ Doppelpuncte $Q_{2}$, so wie $m(m-1)$, auch die Basis berührende, $(m-2)$ fache Tangenten hat, und deren Asymptoten $\mathfrak{A}_{s}$ beziehlich durch die $\frac{1}{2} m(m-1)$ gegenseitigen Schnitte der m Asymptoten $\boldsymbol{A}_{s}$ der Basis gehen, und zu diesen mit der Richtung von $\mathbf{S}$ zugeordnet harmonisch sind, so da/s, wenn etwa $\boldsymbol{A}_{s}$ und $\boldsymbol{B}_{\text {s }}$ swei Asymptoten der Basis sind, welche dieselbe in $a_{\infty}$ und $b_{\infty}$ berühren und irgend eine der parallelen Transversalen $S$ in $a_{1}$ und $b_{1}$ schneiden, dafs dann die aus dem Schnitte $\boldsymbol{A}_{s} \boldsymbol{B}_{s}$ durch die Mitte $Q_{1}$ der Strecke $a_{1} b_{1}$ gezogene Gerade $\mathfrak{A}_{s}$ eine Asymplote der Ortscurve ist und sie in der Mitte $\boldsymbol{Q}_{\infty}$ der Strecke $a_{s} b_{\infty}$ berührt. Übrigens werden alle andere Tangenten der Ortscurve durch eine gleiche Construction erbalten. Denkt man sich in irgend zurei Schnitten, etwa a und $b$, von $\boldsymbol{S}$ und $\boldsymbol{C}^{m}$ die $\mathbf{T}$ angenten $\boldsymbol{A}$ und $\boldsymbol{B}$ der letztern, so berührt die aus dem Schnitt $A B$ durch die Mitte $Q$ der Strecke ab gehende Gerade $\mathfrak{A}$ die Ortscurve in $Q$.

„Bewegt sich die Transversale $\boldsymbol{S}$ insbesondere einer Asymptote $\boldsymbol{A}_{s}$ der Basis parallel, so wird die Ortscurve um einen Grad niedriger, oder vielmehr so besteht sie aus der Asymptote $A_{s}$ und aus einer Curve $\frac{1}{2} m(m-1)-1=\frac{1}{2}(m+1)(m-2)^{t e n}$ Grads, welche die $A_{s} \approx u r(m-2)$ fachen Asymptote hat, und zuar dieselbe im Puncte $a_{\infty}$ mit m-2 Zweigen berührt, die somil einander daselbst ebenfalls berühren."

b. „Giebt man der Transversale $\mathbf{S}$ nacheinander alle Richtungen, so entsteht eine Curven-Schaur, $\boldsymbol{S}\left(Q^{\frac{1}{2 m(m-1)}}\right)$, welche die ganze Ebene so bedecken, da/s durch jeden Punct $P$, im Allgemeinen, je $\frac{1}{2} m(m-1)$ derselben gehen." *) Während die Transversale $\boldsymbol{S}$ ihre Richtung ändert, drehen sich die $\frac{1}{2} \boldsymbol{m}(\boldsymbol{m}-1)$ Asymptoten $\mathfrak{A}_{s}$ der veränderlichen Curve $\boldsymbol{Q}^{\frac{1}{2 m(m-1)}}$ um die festen Schnittpuncte der $m$ Asymptoten $\boldsymbol{A}_{s}$ der Basis und bilden eben

*) Diejenigen Puncte $\boldsymbol{P}$, durch welche eine Curve weniger geht, liegen in der Enveloppe $E^{z}$ der Curven-Schaar, welche von jeder der letztern in $\frac{1}{4} m^{2}(m-1)^{2}$ Puncten berührt wird. Woraus besteht diese Enveloppe $E^{z}$, oder welche Eigenschuften hat dieselbe?' - Besteht sie nicht aus zwei getrennten Theilen, nämlich 1) aus dem Ort aller Doppelpuncte $Q_{2}$ der Curven-Schaar, etwa aus einer Curve $x^{\text {ien }}$ Grads $Q_{2}^{r}$, und zwar diese doppelt genommen; und 2) aus dem Ort der Mitten $Q_{0}$ aller derjenigen einfachen Sehnen $\mathfrak{S}$ der Basis, welche die Berührungspuncle paralleler Tangenten der letztern verbinden (vergl. \$. 15. IV. u. S. 21. I.), [welchen Ort ich als vom $m(m+1)(m-2)^{\text {ten }}$ Grad, also $Q_{0}^{m(m+1)(m-2)}$ gefunden habe]? Demnach bestände die Enveloppe $E^{z}$ aus 
94 2. St einer, algeb. Curven, welche Mittelp. haben, u. Eigens. allgem. algeb. Curven.

soviele unter sich projectivische Strahlenbüschel, welche theils perspectivisch, theils schief liegen, also theils perspectivische Durchschnitte $\boldsymbol{G}$ haben und theils Kegelschnitte $K^{2}$ erzeugen; nämlich jeder Strahlbüschel ist mit $2(m-2)$ andern perspectivisch und mit $\frac{1}{2}(m-2)(m-3)$ befindet er sich in schiefer Lage. Die genannten $\boldsymbol{G}$ und $\boldsymbol{K}^{2}$ haben unter sich ebenfalls mannichfaltige Beziehungen, welche jedoch hier aufser Acht gelassen werden.

III. a. „Wird die Transversale $S$ um einen in ihr beliebig gewählten Pol $\boldsymbol{P}$ herumbewegt, so beschreiben die $\frac{1}{2} m(m-1)$ Milten $\boldsymbol{Q}$ eine Curve $\boldsymbol{m}(m-1)^{\text {sten }}$ Grads $\boldsymbol{Q}^{m(m-1)}$ und $(m-1)^{2} m^{\text {ter }}$ Classe, welche den Pol $\boldsymbol{P}$ zum $\frac{1}{2} m(m-1)$ fachen Punct und nebstdem noch $\frac{3}{8} m(m-1)(m-2)(m-3)$ Doppelpuncte $Q_{2}$ hat, so wie ferner in jedern der $m$ Puncte $a_{\infty}$ der Basis $C^{m}$ sich selbst (m-1) mal berührt, so dafs sie nur m Asymptoten $\mathfrak{A}_{\text {s }}$ hat, aber jede derselben (m-1)fach su sühlen ist, und zwar sind diese Asymptoten beziehlich den Asymptoten $\boldsymbol{A}_{s}$ der Basis parallel, und liegen halb so weit vom Pol ab, als diese, so dafs also die beiden Asymptoteninseit, $m \mathfrak{A}_{s}$ und $m \boldsymbol{A}_{s}$, ähnlich sind, den Pol $\boldsymbol{P}$ zum Ähnlichkeitspunct haben und ihre entsprechenden Dimensionen sich verhalten, wie 1:2." „Liegt der Pol $\boldsymbol{P}$ insbesondere in der Basis selbst, so zerfällt die Ortscurve in zwei Theile $\boldsymbol{Q}^{m}+\boldsymbol{Q}^{m(m-2)}$. Die Curve $\boldsymbol{Q}^{m}$ ist der Basis ähnlich und mit ihr ähnlich liegend; beide berühren einander im Pol $\boldsymbol{P}$, der ihr Älnlichkeitspunct ist, und ihre entsprechenden Dimensionen verhalten sich, wie 1:2; so dafs also ihre Asymptoten parallel sind und nach diesem Verhältnifs vom Pol abstehen. Die andere Curve $\boldsymbol{Q}^{m(m-2)}$ hat den Pol zum $\frac{1}{2}(m+1)(m-2)$ fachen Punct, so wie die m Asymptoten $\mathfrak{A}_{s}$ der ersten Curve $Q^{m}$ zu $(m-2)$ fachen Asymptoten; und nebstdem hat sie noch $\frac{1}{8}(3 m+1)(m-2)(m-3)(m-4)$ Doppelpuncte $Q_{2} . "$

b. Soll die Ortscurve $Q^{m(m-1)}$ durch irgend einen gegebenen Punct $\mathfrak{S}$ gehen und ihren Pol $P$ in einer gegebenen Geraden $G$ haben, so finden $\frac{1}{2} m(m-1)$ Lösungen statt. Oder: Bewegt sich der Pol $\boldsymbol{P}$ in einer festen

$2 Q_{2}^{x}+Q_{0}^{m(m+1)(m-2)}$, und jede Curve $Q^{\frac{1}{2} m(m-1)}$ der obigen Schaar hătte $\frac{1}{8} m(m-1)(m-2)(m-3)$ Doppelpuncte $Q_{2}$ in $Q_{2}^{x}$, was für $\frac{1}{4} m(m-1)(m-2)(m-3)$ Berührungen zählte, und somit müfste der andere Theil, $Q_{0}^{m(m+1)(m-2)}$, von jeder Curve $Q^{\frac{1}{m} m(m-1)}$ in $\frac{1}{2} m(m-1)(2 m-3)$ Puncten berührt werden.

Für die Basis $C^{3}$ bestände $E^{z}$ nur allein aus $Q_{0}^{12}$ (S. 15. IV.) und diese würde von jeder $Q^{3}$ in 9 Puncten $Q_{0}$ berührt.

Für die Basis $C^{4}$, wo $Q_{2}^{z}=Q_{2}^{10}$ (\$. 17.), wäre $E^{z}=2 Q_{2}^{10}+Q_{0}^{40}$, und jede Curve $Q^{6}$ hätte 3 Doppelpuncte $Q_{2}$ in $Q_{2}^{10}$ und berührte die Curve $Q_{0}^{40}$ in 30 Puncten $Q_{0}$. 
2. Steiner, algeb. Curven, welche Mittelp. haben, u. Eigens. allgem. algeb.Curven. 95

Geraden $\boldsymbol{G}$, so ist die ihm entsprechende Curven-Schaar $\boldsymbol{S}\left(\boldsymbol{Q}^{m(m-1)}\right)$ so beschaffen, da/s durch jeden Punct $\mathfrak{Y}$ der Ebene, im Allgemeinen, je $\frac{1}{2} m(m-1)$ derselben gehen, und da/s jede gegebene Gerade $G$ von je $m\left(m^{2}-3\right)$ derselben berührt wird. Die Enveloppe dieser Curven-Schaar enthält dieselben Bestandtheile, wie die vorige (II. b. Note), aber aufserdem noch verschiedene andere Theile.

IV. Die in den vorstehenden Sätzen genannten Doppelpuncte $\boldsymbol{Q}_{2}$ (II. a. u. III. a.) zeigen diejenigen Transversalen $S$ an, in welchen von den $\frac{1}{2} m(m-1)$ Strecken irgend zwei, etwa $a d$ und $b c$, dieselbe Mitte $\boldsymbol{Q}_{2}$ haben, und somit, nach der früheren Erklärung und Bezeichnung, eine durch den jedesmaligen Pol $\boldsymbol{P}$ gehende Doppelsehne $\boldsymbol{S}_{2}$ bilden. Daher:

a. „Der Ort aller Doppelsehnen $\boldsymbol{S}_{2}$ einer gegebenen Curve $m^{\text {ten }}$ Grads $C^{m}$ ist eine Curve $\frac{3}{8} m(m-1)(m-2)(m-3)^{\text {ter }}$ Classe

$$
\boldsymbol{S}_{2}^{\frac{3}{3} m(m-1)(m-2)(m-3)},
$$

welche die Gerade $G_{\infty}$ zur $\frac{1}{4} m(m-1)(m-2)(m-3)$ fachen, so wie auch jede Asymptote der Basis zur vielfachen Tangente hat, *) und welche namentlich auch die $\frac{1}{2} m(m-2)\left(m^{2}-9\right)$ Doppeltangenten der Basis berührt." Die Richtungen, nach welchen die $\frac{1}{4} m(m-1)(m-2)(m-3) \mathrm{Be}-$ rührungspuncte der Ortscurve und der Geraden $\boldsymbol{G}_{\infty}$ liegen, werden erhalten, wenn man zu je 4 der $m$ Asymptoten $\boldsymbol{A}_{s}$ der Basis $\boldsymbol{C}^{m}$ gleicherweise drei Paar harmonische Strahlen $X$ und $X_{1}, \quad Y$ und $Y_{1}, Z$ und $Z_{1}$ construirt, wie oben zu den $4 \boldsymbol{A}_{s}$ der Basis $\boldsymbol{C}^{4}$ (\$.17. S. 48). Durch jeden beliebigen Pol $\boldsymbol{P}$ gehen, im Allgemeinen, $\frac{3}{8} \boldsymbol{m}(\boldsymbol{m}-1)(\boldsymbol{m}-2)(\boldsymbol{m}-3)$ Doppelsehnen $\boldsymbol{S}_{2}$; liegt der Pol in $\boldsymbol{G}_{\infty}$, so sind nur noch ein Drittel derselben wahrnehmbar, indem die übrigen auf $G_{\infty}$ fallen. Liegt der Pol $P$ irgendwo in der Basis, so ist er selbst ein Endpunct, etwa a, von $\frac{1}{2}(2 m+1)(m-2)(m-3)$ Doppelsehnen, deren Mitten $\boldsymbol{Q}_{2}$ in der obigen Ortscurve $\boldsymbol{Q}^{m}$ liegen (III. a.), so wie auch in einer andern Curve $(m-1)(m-3)^{\text {ten }}$ Grads, welche die $\boldsymbol{Q}^{m}$ im Pol $\frac{1}{2}(m+2)(m-3)$ punctig berührt.

b. „Der Ort der Mitten $\boldsymbol{Q}_{2}$ aller Doppelsehnen $\boldsymbol{S}_{2}$ der gegebenen Basis $C^{m}$ ist eine Curve $\frac{1}{4} m(m+1)(m-2)(m-3)^{\text {ten }}$ Grads

$$
Q_{2}^{\frac{2}{4} m(m+1)(m-2)(m-3)} \text {, }
$$

*) $\mathrm{Zu}$ wievielfachen Tangenten hat sie jede Asymptote $\boldsymbol{A}_{s}$ der Basis? Etwa zu $(m-2)(m-3)$ fachen? und berührt sie jede $\frac{1}{2}(m-2)(m-3)$ mal im Puncte $a_{\infty}$, so dafs sie dieselbe zugleich zur $\frac{1}{2}(m-2)(m-3)$ fachen Asymptote hat, und nebstdem in eben sovielen, nicht im Unendlichen liegenden Puncten berührt? 
96 2. St einer, algeb. Curven, welche Mittelp. haben, u. Eigens. allgem. alyeb. Curven.

welche die Asymptoten $A_{s}$ der Busis zu vielfachen $\left[\frac{1}{2}(m-2)(m-3)\right.$ fachen?] Asymptoten hat, und die Gerade $G_{\infty}$ nebstdem in denselben Puncten schneidet, in denen diese von der Ortscurve der $S_{2}$ berüht uird, und welche ferner insbesondere auch durch die Mitten der Doppeltangenten der Basis geht."

Liegt von den $\frac{1}{2} m(m-1)$ Mitten $\boldsymbol{Q}$ einer Transversale $\boldsymbol{S}$,irgend eine, die $Q_{1}$ heifsen soll, in der Basis selbst (ohne dafs die zugehörige Strecke $=0$ ist), z. B. liegt die Mitte $\boldsymbol{Q}_{\mathbf{1}}$ der Strecke $a c$ im Schnitt $b$, und wird dabei, wie früher ( $\$$. 15. II.), die Transversale oder die einfache Sehne $a c$ durch $\boldsymbol{S}_{\mathbf{1}}$ bezeichnet, so ergiebt sich durch die obigen Sätze ferner leicht der folgende Satz.

c. „Der Ort aller einfachen Sehnen $\boldsymbol{S}_{1}$ der gegebenen Basis $\boldsymbol{C}^{m}$, deren Mitten $Q_{1}$ in der Basis selbst liegen, ist eine Curve $m(m-1)(m-2)^{\text {ter }}$ Classe

$$
\boldsymbol{S}_{1}^{m(m-1)(m-2)}
$$

welche die Gerade $G_{\infty}$ zur $\frac{1}{2} m(m-1)(m-2)$ fachen Tangente, so wie auch die m Asymptoten $A_{s}$ der Basis zu 2(m-2)fachen Tangenten und zu (m-2)fachen Asymptoten hat, und welche die Basis in ihren $3 m(m-2)$ Wendepuncten berührt." Die Richtungen, nach welchen die Berührungspuncte dieser Curve und der Geraden $\boldsymbol{G}_{\infty}$ liegen, sind gleicherweise durch die Asymptoten der Basis bestimmt, wie früher bei der Basis $\boldsymbol{C}^{3}$ (\$. 15. II. 5.); nämlich die in jedem durch irgend drei Asymptoten der Basis $\boldsymbol{C}^{m}$ gebildeten Dreieck aus den Ecken durch die Mitten der Gegenseiten gezogenen drei Strahlen sind nach drei jener Berührungspuncte gerichtet. Eben so ist der Berührungspunct, $s$, jeder Sehne $S_{1}$ mit der Ortscurve, hier auch durch dieselbe einfache Construction zu finden, wie dort (\$. 15. II. 7.). - Durch jeden beliebigen Pol $P$ gehen $m(m-1)(m-2)$ Sehnen $S_{1}$ : ihre $m(m-1)(m-2)$ Mitten $Q_{1}$ liegen allemal in irgend einer Curve $(m-1)(m-2)^{\text {ten }}$ Grads $\boldsymbol{Q}_{1}^{(m-1)(m-2)}$. Liegt der Pol $\boldsymbol{P}$ in der Basis selbst, so ist er einerseits die Mitte $b=Q_{1}$ von $\frac{1}{2}(m+1)(m-2)$ Sehnen $a c=S_{1}$, und andererseits ein Endpunct a von $(m+1)(m-2)$ andern Sehnen $S_{1}$ : die $(m+1)(m-2)$ Mitten der letztern liegen in der obigen Curve $\boldsymbol{Q}^{n}$ (III.a.), welche die Basis in $\boldsymbol{P}$ berührt. Liegt ferner der Pol $\boldsymbol{P}$ im Unendlichen, in $\boldsymbol{G}_{\infty}$, so sind nur noch $\frac{1}{2} m(m-1)(m-2)$ Sehnen $S_{1}$ wahrnehmbar (indem eben soviele auf $G_{\infty}$ fallen), und durch ihre Mitten $Q_{1}$ können Curven $Q_{1}^{t(m-1)(m-2)}$ gehen. Bewegt sich $\boldsymbol{P}$ in der Geraden $\boldsymbol{G}_{\infty}$; so entsteht eine Curven-Schaar $\boldsymbol{S}\left(\boldsymbol{Q}_{1}^{\frac{1}{(}(m-1)(m-2)}\right)$. So oft eine dieser Curven die Busis berührt, wobei zwei 
2. Steiner, algeb. Curven, welche Mittelp. haben, u. Eigens. allgem. algeb. Curven. 97

der genannten Sehnen $\mathcal{S}_{1}$ in eine, $\boldsymbol{S}_{1}^{(0)}$, zusammenfallen, ist diese eine Asymptote der Curve $\boldsymbol{S}_{1}^{m(m-1)(m-2)}$ und berüht sie im entsprechenden Pol P; zudem hat jede solche Sehne $\mathcal{S}_{1}^{0}$ die Eigenschuft, da/s die in ihren Endpuncten a, $c$ und in ihrer Mitte $b$ an die Basis gelegten Tangenten $A, C$ und $B$ sich in irgend einem Puncte $\mathfrak{P}$ treffen. - 1) Welche Enveloppe hat die Curven-Schuar $\boldsymbol{S}\left(Q_{1}^{(m-1)(m-2)}\right)$ ? 2) Von welchem Grad ist die Curve $\mathbf{S}_{1}^{m(m-1)(m-2)}$ ?

V. Es folgt weiter:

a. „Der Ort aller Transversalen $S$ in Bezug auf die gegebene Basis $C^{m}$, welche eine ihrer Mitten $\boldsymbol{Q}$ in einer gegebenen Geraden $\boldsymbol{G}$ haben, oder schlechthin, der Ort aller einfachen Sehnen $a b=S$, deren Mitten in der gegebenen Geraden $G$ liegen, ist eine Curve $m(m-1)^{\text {ster }}$ Classe

$$
S^{m(m-1)}
$$

und $m\left(m^{2}-3\right)^{\text {ten }}$ Grads, welche die Geraden $G$ und $G_{\infty} \approx u \frac{1}{2} m(m-1)-$ fachen Tangenten, so wie sudem noch $\frac{1}{4} m(m+1)(m-2)(m-3)$ Doppeltangenten hat, und welche insbesondere auch die $m$ Asymptoten der Basis nebst deren $m$ Tangenten in ihren Schnitten mit $G$, so wie ferner auch die Basis selbst in $m(m+2)(m-2)$ bestimmten Puncten berührt." Nümlich die genannten Doppeltangenten bestehen aus denjenigen Doppelsehnen $\mathbf{S}_{2}$ der Basis, deren Mitten $Q_{2}$ in $G$ liegen, und die Ortscurve berührt die Basis in den Berührungspuncten $a_{0}$ aller derjenigen Tangenten-Sehnen ba $_{01}$ (\$. 21. I.), welche ihre Mitte gleichfalls in $G$ haben. Die Ortscurve berührt die Gerade $G$ in $\frac{1}{2} m(m-1)$ Puncten und schneidet sie also noch in $m(m+1)(m-2)$ andern Puncten $Q_{0}$ : ihre Tangenten in diesen Puncten $Q_{0}$ sind solche besondere Sehnen $a b=\mathfrak{S}(=\mathbb{S})$, in deren Endpuncten $a, b$ die Tangenten $A, B$ an die $B$ asis parallel sind und sich in einem Puncte auf $G_{\infty}$ treffen; und gleicherweise sind die Tangenten der Ortscurve in ihren $m(m+1)(m-2)$ Schnitten mit der Geraden $G_{\infty}$, also ihre geradlinigen Asymptoten, solche Sehnen ab, in deren Endpuncten die Tangenten an die Basis sich auf der Geraden $G$ treffen, so da/s also in diesem Betracht zwischen $G$ und $G_{\infty}$ Reciprocitait statt findet.

b. „Der Ort aller derjenigen Transversalen $S$ der gegebenen Basis $C^{m}$, welche eine ihrer Mitten $\boldsymbol{Q}$ in einer gegebenen Curve $n^{\text {ten }}$ Grads $G^{n}$ haben, ist eine Curve $n$ in $(m-1)^{\text {ster }}$ Classe, welche die Gerade $G_{\infty}$ zur $\frac{1}{2} n m(m-1)$ fachen Tangente hat, und von welcher ferner angegeben

Crelle's Journal f. d. M. Bd. XLVII. Heft 1. 
98 2. St einer, algeb. Curven, welche Mittelp. haben, u. Eigens. allgem. algeb. Curven.

werden kann, wieviele Doppeltangenten sie habe, wie oft sie die Curven $C^{m}$ und $G^{n}$ berühre, u. s. w."

c. „Der Ort der $\frac{1}{2} m(m-1)$ Milten $Q$ derjenigen Tarsversule $\boldsymbol{S}$ der Basis $C^{m}$, welche eine gegebene Curve $n^{\text {ter }}$ Classe $K^{n}$ berührt, ist eine Curve $n m(m-1)^{\text {sten }}$ Grads, welche mit jeder der m Asymptoten der Basis $n$ parallele aber zugleich (m-1)fache Asymptoten hat, u. s. w."

d. „Der Ort der Mitten $Q_{1}$ aller solchen einfachen Sehnen $a b=\mathfrak{S}$ der Basis $C^{m}$, in deren Endpuncten $a, b$ die Tangenten $A, B$ parallel sind, oder der Ort desjenigen Pols $Q_{1}$, dessen innere Polare $J^{m-1}$ die Basis in irgend zwei Puncten $a, b$ berührt (\$. 21. I.), ist eine Curve $m(m+1)(m-2)^{\text {ten }}$ Grads

$$
\boldsymbol{Q}_{0}^{m(m+1)(m-2)},
$$

welche die Basis in ihren $3 m(i n-2)$ Wendepuncten berührt und ihre m Puncte $a_{s} z u(m+1)(m-2)$ fachen Puncten, also mit jeder $\boldsymbol{A}_{s}$ der Basis eben soviele parallele Asymptoten hat, die beziehlich in der Mitte zwischen $A_{s}$ und den mit ihr parallelen Tangenten der Basis liegen." Demzufolge giebt es, im Allgemeinen, $m(m-2)\left(m^{2}-7\right)$ solche Sehnen $\mathfrak{S}$, welche ihre Mitte $\boldsymbol{Q}_{0}$ in der Basis selbst, aber weder in einem der Puncte $\boldsymbol{a}_{\infty}$, noch in einem Wendepunct derselben haben. (Für $m=3$ kommt $6 \mathfrak{S}$ oder $6 \boldsymbol{Q}_{0}$, wie \$. 15. IV.) Hier entsteht die Frage: Welches ist der Ort, $\mathfrak{S}^{x}$, aller Sehnen $\left.\mathfrak{S}^{*}{ }^{*}\right)$ Der Berührungspunct jeder Sehne $\subseteq$ mit der Ortscurve $\mathfrak{S}^{x}$ ist übrigens durch dieselbe einfache Bedingung bestimmt, wie oben ( $\$$. 15. IV.) bei der Basis $C^{3}$.

VI. Der Ort der Mitten $\boldsymbol{Q}$ aller Transversalen $\boldsymbol{S}$, welche die Basis $\boldsymbol{C}^{m}$ berühren, also aller Tangenten der letztern, zerfällt in drei Theile, wovon der eine die Basis selbst ist und nur die im Berührungspunct, etwa $a_{1)}$, liegende eine Mitte enthält; dagegen enthält ein anderer Theil die Mitten, etwa $\boldsymbol{T}_{0}$ (statt $\boldsymbol{Q}$ ), derjenigen $\boldsymbol{m}-2$ Strecken, welche zwischen dem Berührungspunct $\boldsymbol{a}_{0}$ und den $m-2$ Schnitten $b, c, d, \ldots$ liegen (wobei eigentlich in jedem $\boldsymbol{T}_{0}$ ein Paar $\boldsymbol{Q}$ vereint sind (I.)); und der dritte Theil enthält die Mitten $\boldsymbol{T}(=\boldsymbol{Q})$ der von diesen $m-2$ Schnitten begrenzten Strecken. Somit liegen in jeder Tangente $m-2$ Mitten $T_{0}$ und $\frac{1}{2}(m-2)(m-3)$ Mitten $T$; ihre respectiven Örter aber sind folgende.

*) Bei einem Versuch, diesen Ort zu bestimmen, fand ich $x=\frac{1}{2} m(m-1)(2 m-3)$. 
2. Steincr, algeb. Curven, welche Mittelp. haben, u. Eigens. allgem. algeb. Curven. 99

a. ,Der Ort der Mitten $\boldsymbol{T}_{0}$ rücksichtlich aller Tangenten der gegebenen Basis $C^{m}$ ist eine Curve $m(m+2)(m-2)^{t e n}$ Grads

$$
\boldsymbol{T}_{0}^{m\left(m^{2}-4\right)},
$$

welche die $m$ Puncte $a_{\infty}$ der Basis zu $m(m-1)$ fachen Puncten und jede $\boldsymbol{A}_{s}$ derselben zur (m-2)fachen Asymptote hat, d. h., welche jede $\boldsymbol{A}_{s}$ der Basis in deren Punct $a_{\infty}$ mit $m-2$ Zweigen berührt und mit $(m+1)(m-2)$ indern Zweigen schneidet, und welche ferner mit der Basis deren $3 m(m-2)$ Wendepuncte und Wendetangenten gemein hat, so wie jede Doppeltangente derselben in ihrer Mitte berührt." Daraus folgt: ,Eine beliebige Curve $C^{m}$ hat, im Allgemeinen, $m(m+4)(m-2)(m-3)$ solche Tangenten, bei uelchen ein Schnittpunct $b$ in der Mitte zwischen dem Berührungspunct $a_{11}$ und einem andern Schnittpunct c liegt."

$\beta$. ,Der Ort der Mitten $\boldsymbol{T}$ aller Tangenten der Basis $C^{m}$ ist eine Curve $m(m+1)(m-2)(m-3)^{\text {ten }}$ Grads

$$
\boldsymbol{T}^{m(m+1)(m-2)(m-3)},
$$

welche die $m$ Puncte $a_{\infty}$ der Basis zu $(m+1)(m-2)(m-3)$ fachen Puncten hat, durch die Berührungspuncte ihrer $\frac{1}{2} m(m-2)\left(m^{2}-9\right)$ Doppeltungenten geht und nebstdem jede dieser Doppeltangenten in $2(m-4)$ Puncten berührt.” Folgerung: ,Eine beliebige Curve $C^{m}$ hat, im Allgemeinen, $m(m-2)(m-3)\left(m^{2}-m-4\right)$ solche Tangenten, bei welchen von den $m-2$ Schnitten irgend zwei, $c$ und $d$, gleichweit von einem dritten, $b$, oder vom Berührungspunct, $a_{0}$, abstehen; wobei jedoch der letztere Fall für 2 zu sühlen ist."

Für die Basis $\boldsymbol{C}^{3}$ hat man als Ort der Mitten $\boldsymbol{T}_{0}$ aller Tangenten eine Curve $\boldsymbol{T}_{10}^{12}(=12)$ wie oben ( $\$$. 15. IV.).

Bei der Basis $C^{4}$ sind die Ortscurven $\boldsymbol{T}_{0}^{48}$ und $\boldsymbol{T}^{40}$, und es folgt:

$\left(\alpha^{0}.\right)$,Eine beliebige Curve $4^{\text {ten }}$ Grads $C^{4}$ hat, im Allgemeinen, 64 solche Tangenten, bei welchen einer der beiden Schnitte, etwab, in der Mitte zwischen dem andern $c$ und dem Berührungspuncte $a_{0}$ liegt." Und

( $\left.\beta^{\circ}.\right)$, ,Dieselbe Curve mu/s $64: 2=32$ solche Tangenten haben, bei welchen die beiden Schnitte $b$ und $c$ gleichweit vom Berührungspunct $a_{0}$ abstehen (wie \$. 17.)." Also: Die Ortscurve $T^{41}$ hat die 4 Puncte $a_{\infty}$ der Basis $C^{4}$ zu 10 fachen Puncten, geht durch die 56 Berührungspuncte ilerer 28 Doppeltangenten und berührt dieselbe in den oben näher beschriebenen 32 Puncten $\boldsymbol{P}^{0}$ (\$. 17.). 
100 2. St einer, algeb. Curven, welche Mittelp. haben, u. Eigens. allgem. algeb. Curven.

Ist $m>4$, so wird die Basis $\boldsymbol{C}^{m}$ von der Ortscurve der $\boldsymbol{T}$ (aufser den im Satze ( $\beta$.) namhaft gemachten Puncten, noch) in $x$ Puncten berührt und in $y$ Puncten geschnitten, wobei

$$
2 x+y=m(m-2)(m-3)\left(m^{2}-m-4\right)
$$

sein mufs: Diese zwei Zahlen $x$ und $y$ zu finden? [Ist nicht $y=(m-4) x$ ? wie ein gewisser Wahrscheinlichkeits-Grund es erheischt. Dann wäre

$$
x=m(m-3)\left(m^{2}-m-4\right) \quad \text { und } y=m(m-3)(m-4)\left(m^{2}-m-4\right) \text {, }
$$

und die Basis $C^{m}$ hätte $m(m-3)\left(m^{2}-m-4\right)$ solche Tangenten, bei welchen zwei Schnitte $c$ und d gleichweit vom Berührungspunct $a_{1}$ abständen, und ferner $m(m-3)(m-4)\left(m^{2}-m-4\right)$ solche Tangenten, bei welchen ein Schnitt $b$ in der Mitte zwischen zwei andern $c$ und d läge.]

VII. In der gegebenen Basis $\boldsymbol{C}^{m}$ giebt es auch solche besondere Transversalen, bei welchen der Schwerpunct $A$ ihrer $m$ Schnitte (\$.25.) mit einer ihrer $\frac{1}{2} m(m-1)$ Mitten $\boldsymbol{Q}$ zusammenfällt. Jede solche Transversale heifse $\boldsymbol{S}_{a}$ und ihr Schwerpunct $\boldsymbol{Q}_{a}$; so sind die respectiven Örter derselben

$$
\boldsymbol{S}_{a}^{\frac{1}{1} m(m-1)^{2}} \text { und } \boldsymbol{Q}_{a}^{\frac{1}{t} m(m+1)(m-2)},
$$

d. h.: ,Bei einer beliebigen Curve $C^{m}$ ist der Ort derjeniyen Transversale $\boldsymbol{S}_{a}$, deren Schwerpunct $\boldsymbol{Q}_{a}$ in der Mitte zwischen irgend zwei Schnitten liegt, eine Curve $\frac{1}{2} m(m-1)(m-1)^{\text {ster }}$ Classe und der Ort des Schwerpuncts ist eine Curve $\frac{1}{2} m(m+1)(m-2)^{\text {ten }}$ Grads."

Für die Basis $C^{3}$ sind danach die Ortsurven: $\boldsymbol{S}_{a}^{6}$ und $\boldsymbol{Q}_{a}^{6}$; die erste ist die obige Curve $\boldsymbol{S}_{1}^{6}$ ( $\left(\mathbf{S}\right.$. 15. II.), und die andere bedeutet die doppelte Basis $\boldsymbol{C}^{3}$, indem in der That jeder Punct in $C^{3}$ die Mitte $\left(=Q_{a}\right)$ zweier Sehnen $\boldsymbol{S}_{1}$ ist ( $\$$. 15.).

Bei der Basis $\boldsymbol{C}^{4}$ sind die Ortscurven: $\boldsymbol{S}_{a}^{18}$ und $\boldsymbol{Q}_{a}^{20}$; aber jede ist eine doppelte Curve, indem hier jede Transversale $\boldsymbol{S}_{a}$ eine Doppelsehne $\boldsymbol{S}_{2}$ ist, und daher zwei Mitten $\boldsymbol{Q}$ im Schwerpuncte $\boldsymbol{Q}_{a}$ liegen; die einfachen Örter sind somit nur $\boldsymbol{S}_{a}^{9}$ und $\boldsymbol{Q}_{a}^{\mathbf{1 0}}$; wie wir sie bereits aus $\$$. 17. kennen. - Für $m>4$ hören diese Reductionen der Ortscurven auf.

Bei $\cdot$ der Basis $C^{5}$ hat man: $S_{a}^{41}$ und $\boldsymbol{Q}_{a}^{45}$. Die Basis hat mit der ersten 800 Tangenten $\boldsymbol{S}_{a}^{\prime \prime}\left(=\boldsymbol{S}_{a}\right)$ und mit der andern 225 Puncte $\boldsymbol{Q}_{a}^{\prime \prime}\left(=\boldsymbol{Q}_{a}\right)$ gemein. Nimmt man an, $\boldsymbol{Q}_{a}$ liege in der Mitte zwischen den Schnitten $a$ und $\boldsymbol{b}$, so ist er zugleich der Schwerpunct der drei übrigen Schnitte $c, d$ und $e$; und wird der Berührungspunct jeder $\boldsymbol{S}_{a}^{0}$ mit $\boldsymbol{C}^{\mathbf{5}}$ durch $\boldsymbol{B}_{0}$ bezeichnet, so können folgende verschiedene Umstände stalt finden. 
A. In Betreff der $800 \boldsymbol{S}_{a}^{0}$ sind drei Fälle möglich, entweder liegen:

a) etwa $c$ und $d$ (oder ce oder $d e$ ) in $\boldsymbol{B}_{0}$, oder

$\beta$ ) etwa $a$ und $e$ (oder $a c, a d, b c, b d, b e$ ) in $\boldsymbol{B}_{0}$, oder

$\gamma) a$ und $b$ in $B_{0}$ und somit auch $\boldsymbol{Q}_{a}^{0}$ in $\boldsymbol{B}_{0}$; und

B. In Betracht der $225 \boldsymbol{Q}_{a}^{0}$ sind 2 Fälle möglich; entweder liegt:

ס) $Q_{a}^{\prime \prime}$ in $e$ (oder $c, d$ ) und ist nicht allein die Mitte von $a b$, sondern auch von $c d$, so dafs die zugehörige $\boldsymbol{S}_{a}=\boldsymbol{S}_{2}$ wird, oder

' $\varepsilon) \boldsymbol{Q}_{a}^{\prime}$ in $a$ und $b$ vereint, also in $\boldsymbol{B}_{0}$, wie Fall $(\alpha$.$) , so dafs die$ zugehörige $\boldsymbol{S}_{a}=\boldsymbol{S}_{a}^{0}$ wird, d. h. die $\boldsymbol{C}^{5}$ in $(a b)=Q_{a}^{\text {j- berührt. }}$

Dabei entsteht die Frage: Wie oft tritt jeder dieser Fälle ein? und namentlich: Wieviele der 225 Puncte $Q_{a}^{\prime \prime}$ gehören dem Falle $(\delta)$ und wieviele dem Falle $(\varepsilon)$ an? Der Fall $(\delta)$ enthält die oben (\$.19.) verlangten Puncte, und bestätigt die dortige Angabe über ihre Anzahl. - Analoge Fragen sind bei der allgemeinen Basis $\boldsymbol{C}^{m}$ zu stellen.

\section{\$. 27.}

Durch Projection gehen die vorigen Sätze (\$. 26.) in solche andere Sätze über, bei welchen die betrachteten Mitten $\boldsymbol{Q}$ durch gewisse harmonische Puncte $\boldsymbol{N}$ vertreten werden, nämlich bei welchen nebst $\operatorname{der}$ Basis $\boldsymbol{C}^{m}$ noch irgend eine Gerade $\boldsymbol{G}$ gegeben ist (dort war es $\boldsymbol{G}_{\infty}$ ), und wobei dann zu dem Schnitt $\boldsymbol{R}$ der Transversale $\boldsymbol{S}$ und dieser Geraden $\boldsymbol{G}$ in Bezug auf je zwei der, $m$ Schnitte $a, b, c, d, \ldots$ von $\boldsymbol{S}$ und $\boldsymbol{C}^{m}$ der vierte harmonische Punct $\boldsymbol{N}$ genommen wird.

Es können aber in der Transversale ferner auch harmonische Puncte auf andere Weise bestimmt werden, wie z. B. so: dafs man zu je drei ihrer $m$ Schnitte $a, b, c, d, \ldots$ mit $\boldsymbol{C}^{m}$ die drei vierten harmonischen Puncte $\boldsymbol{N}$ nimmt (\$. 11. III.), wodurch man in jeder Transversale im Ganzen $\frac{1}{2} m(m-1)(m-2)$ Puncte $\mathbf{N}$ erhält. Und wird sodann die Transversale geeigneten Bedingungen unterworfen, so gelangt man zu neuen Curven, so wie zu neuen Eigenschaften der Basis $\boldsymbol{C}^{m}$. Ich mufs mich jedoch darauf beschränken, hier nur einige leichtere Resultate kurz mitzutheilen.

I. Es kann verlangt werden, dafs von den $m$ Schnitten $a, b, c, d, \ldots$ irgend vier unter sich harmonisch sein sollen. Dies führt zu dem folgenden Satze.

„Der Ort derjenigen Transversale $\mathbf{S}$, welche eine gegebene Curve $m^{\text {ten }}$ Grads $C^{m}$ in irgend 4 harmonischen Puncten schneidet, ist eine Curve $\frac{1}{4} m(m-1)(m-2)(m-3)^{\text {ter }}$ Classe

$$
\boldsymbol{S}^{\frac{1}{m} m(m-1)(m-2)(m-3)}
$$


102 2. St ein er, algeb. Curven, welche Mittelp. haben, u. Eigens, allgem. algeb. Curven.

welche die Basis in jedem ihrer Wendepuncte mit je m-3 Zweigen, so wie nebstdem (wenn $m>4$ ) noch in vielen andern Puncten berührt." Der Berührungspunct jeder $\boldsymbol{S}$ mit der Ortscurve ist durch Hülfe der in den vier harmonischen Schnittpuncten an die Basis gelegten vier Tangenten leicht zu construiren. Aufgabe: Den Grad der Ortscurve su bestimmen.

Bei der Basis $C^{4}$ ist demnach der Ort der Geraden $S$, welche dieselbe in 4 harmonischen Puncten abcd schneidet, eine Curve $6^{\text {ter }}$ Classe $\boldsymbol{S}^{6}$, welche die Basis in ihren 24 Wendepuñcten berührt. Die $12 \cdot 6=7.2$ gemeinschaftlichen Tangenten beider Curven bestehen daher nur aus den 24 Wendetangenten der Basis, indem jede für 3 zählt. Die Curve $\boldsymbol{S}^{6}$ ist vom 30 $^{\text {sten }}$ Grad; sie hat daher mit der Basis, au/ser jenen 24 Berührungspuncten, noch 72 Puncte (Schnitte) $\left.a_{0}=a\right)$ gemein, und ihre Tangente $\mathbf{S}$ in jedem dieser $a_{0}$ schneidet die Basis, au/ser daselbst, in drei solchen Puncten b, $c$, d (die mit $a_{0}$ harmonisch sind und) deren zugehörige Tangenten $B, C, D$ sich in irgend einem Puncte $p$ treffen. Durch die 72 Puncte $a_{0}$ können Curven $18^{\text {ten }}$ Grads gehen. „Welche Lage haben die 72 Puncte $p$ ?" - Besteht insbesondere die Basis $C^{4}$ aus 4 Geraden $A, B, C$ und $D$, so zerfält die Ortscurve $\boldsymbol{S}^{6}$ in die dem Vierseit $\boldsymbol{A B C D}$ eingeschriebenen drei harmonischen Kegelschnitte. (Systemat. Entw, der Abhäng. geom. Gestalten. \$. 43.) Gleicherweise ergeben sich specielle Resultate, wenn die Basis $C^{4}$ aus $C^{2}+2 C^{1}$, oder $C^{2}+C_{1}^{2}$, oder $C^{3}+C^{1}$ besteht.

Bei der Basis $C^{5}$ ist die Ortscurve $=\mathbf{S}^{30}$; sie berührt die Basis in jedem ihrer 45 Wendepuncten mit zwei Zweigen, und nebstdem berührt sie dieselbe noch in 165 andern Puncten a. Daher:

„Eine beliebige Curve $5^{\text {ten }}$ Grads hat, im Allgemeinen, 165 solche harmonische Tangenten, bei welchen der Berührungspunct a und die drei Schnittpuncle b, c, d harmonisch sind."

Bei der Basis $C^{m}$ findet man auf diese Weise, aufser den Wendetangenten,

$$
\frac{1}{4} m(m-2)(m-3)\left[m(m-1)^{2}-36\right]
$$

solche Tangenten, bei welchen von den $m-1$ Puncten, nämlich dem Berührungspunct $a$ und den $m-2$ Schnitten $b, c, d, \ldots$, irgend 4 harmonisch sind, wobei jedoch jeder Fall, wo sich $a$ unter den harmonischen Puncten befindet, für 2 zu zäblen ist, so dafs, wenn die Zahl der Fälle, welche $a$ enthalten, durch $x$ und die ohne $a$ durch $y$ bezeichnet werden, dann $2 x+y$ der vorstehenden Zahl gleich ist. Dabei wird die Basis von der Ortscurve in den $x$ Puncten $a$ berührt. - Diese Zahlen $x$ und $y$ zu bestimmen. 
2. Ste iner, algeb. Curven, welche Mittelp. haben, u. Eigens. allgem. algeb.Curven. 103

II. Wird die gegebene Basis $C^{4}$ von einer Tangente $S$ in $a$ berührt und in $b, c$ geschnitten, und man denkt sich zu diesen 3 Puncten die 3 vierten harmonischen Puncte $\alpha, \beta, \gamma$ und zwar so, dafs

harmonisch sind:

$$
a b \alpha c ; a b c \beta ; \quad a \gamma b c
$$

„So ist der Ort des Puncts a eine Curve $32^{\text {sten }}$ Grads, welche die Basis in ihren Wendepuncten dreipunctig beruhrt und durch die Berührungspuncte ihrer Doppeltangenten geht (s. S. 47 Note)." Und

„So ist der gemeinsame Ort der beiden Puncte $\beta$ und $\gamma$ eine Curve $64^{\text {sten }}$ Grads, welche die Basis in jedem ilser 24 Wendepuncte mit swei Zweigen dreipunctig, so wie in jedem der 56 Berührungspuncte ihrer 28 Doppeltangenten (zweipunctig) berührt. Daraus folgt ferner:

,Da/s die Curve $4^{\text {ten }}$ Grads $C^{4}$, im Allgemeinen, 64 solche Tangenten hat, bei welchen der eine Schnittpunct, $b$, in der Mitte zwischen dem andern, $c$, und dem Berührungspunct, a, liegt (wie \$. 26. VI. $\alpha^{\circ}$ ), und da/s diese besondern Tangenten den Asymptoten der genannten Curve 64 $^{\text {sten }}$ Grads parallel sind."

Wird die gegebene Basis $C^{5}$ von einer Tangente $\boldsymbol{S}$ in $a$ berührt und in $b, c, d$ geschnitten und man bestimmt die drei Puncte $\delta, \gamma, \beta$ so, dafs harmonisch sind: $a b \delta c ; a b \gamma d ; a c \beta d$

So ist der gemeinsume Ort der 3 Puncte $\beta, \gamma, \delta$ eine Curve $x^{\text {ten }}$ Grads, welche die Basis in jedern ihrer 45 Wendepuncte mit zwei Zweigen dreipunctig berührt und dieselbe in den $165 \cdot 3=495$ Schnitten $(b, c, d)$ der vorgenannten 165 harmonischen Tangenten (I.), so wie in den 240 Berührungspuncten ihrer 120 Doppeltangenten schneidet, und welche ferner auch diese Doppeltangenten doppelt berührt. Jede Tangente der Basis, bei welcher irgend zwei der drei Schnitte b, c und d gleichweit vom Berührungspunct a abstehen, ist einer Asymptote der genannten Ortscurve parallel. - Den Grad $x$ zu bestimmen.

III. Durch jede 4 in einer Geraden liegenden Puncte, nach ihrer Reihenfolge $a, b, c$ und $d$, sind drei verschiedene (von mir sogenannte) Puncten-Systeme (Involutions-Systeme) bestimmt, indem man dieselben auf drei Arten als zwei Paar conjugirte Puncte ansehen kann, nämlich
1. $a b$ und $c d$;
2. $a d$ und $b c$;
3. $a c$ und $h d$.

Die zu beiden Paaren jedes Systems gehörigen harmonischen Puncte, bezieh- 
104 2. St ciner, algeb. Curven, welche Mittelp. haben, u. Eigens. allgem. algeb.Curven.

lich $x$ und $x_{1}, y$ und $y_{1}, z$ und $z_{1}$, wobei $a x b x_{1}$ und $c x d x_{1}, a y d y_{1}$ und bycy,$a z c z_{1}$ und $b z d z_{1}$ harmonisch sind ( $\$$. 17.), habe ich, AsymptotenPuncte" und die beiden ersten Systeme, bei denen die Asymploten-Puncte reell sind, „hyperbolisch" dagegen das dritte (3.), bei welchem dieselben imaginär sind, „elliptisch" genannt.

Denkt man sich zu je 4 der m Puncte $a, b, c, d, \ldots$, welche die gegebene Basis $C^{m}$ mit irgend einer Transversale, $\boldsymbol{S}$ gemein hat, die drei Paar Asymptoten-Puncte $x$ und $x_{1}, y$ und $y_{1}, z$ und $z_{1}$, so hat man im Ganzen $\frac{1}{8} m(m-1)(m-2)(m-3)=\mu$ Paare, oder $\frac{1}{4} m(m-1)(m-2)(m-3)=2 \mu$ einzelne Asymptoten-Puncte, von denen jeder durch $\boldsymbol{X}$ bezeichnet werden soll.

,Wird die Transversale $\mathbf{S}$ um einen in ihr beliebig gewählten Pol $\boldsymbol{P}$ herumbewegt, so beschreiben die $2 \mu$ Puncte $X$ insgesammt eine Curve $3 u^{\text {ten }}$ Grads

$$
\boldsymbol{X}^{\frac{3}{8} m(m-1)(m-1)(m-2)(m-3)},
$$

welche den Pol $\boldsymbol{P}$ zum ufachen Punct hat, u. s. w."

Es kann solche besondere Transversalen $\dot{S}_{x}(=S)$ geben, bei welchen ein Asymptoien-Punct mit einem ihrer $m$ Schnittpuncte zusammenfällt, z. B. der Schnitt $e$ kann $x$ sein, so dafs a $e b x_{1}$ und $c e d x_{1}$ harmonisch sind, also das durch die Paare $a$ und $b, c$ und $d$ bestimmte Puncten-System den Schnitt $e$ zum Asymptoten-Punct hat, oder diese 5 Schnitte Involution bilden.

,Der Ort derjenigen Transversale $\boldsymbol{S}_{x}$, bei welcher ein Asymptoten-Punct $\boldsymbol{X}$ in der Basis $C^{m}$ selbst liegt, oder von deren $m$ Schnitten irgend 5 Involution bilden, ist eine Curve

$$
\boldsymbol{S}_{x}^{3} m(m-1)(m-2)(m-3)(m-4), "
$$

Rücksichtlich aller dieser Transversalen $\boldsymbol{S}_{x}$, welche einen AsymptotenPunct $x$ in einem Schnitt $e$ (aber nicht in einem Berührungspunct) der Basis haben, kann gefragt werden: Welchen Ort der dem $x(=e)$ zugehörige andere Asymptoten-Punct $x_{1}$ habe? Dieser Ort wird irgend eine Curve $n^{\text {ter }}$ Grads $\boldsymbol{X}_{1}^{n}$ sein; ihre Schnitte mil der Basis $\boldsymbol{C}^{m}$ bestimmen diejenigen einzelinen Transversalen $\boldsymbol{S}_{x x_{1}}$, welche ein Paar conjugirte AsymptotenPuncte $x$ und $x_{1}$ in der Basis haben. Die Beantwortung der Aufgabe wird erleichtert, wenn zuvor die folgende gelöst ist: Wenn in jeder Tangente $\mathbf{S}$ der Basis $C^{m}$ zu dem Berührungspunct a in Bezug auf je zwei der m-2 Schnitte b, $c, d, \ldots$ der vierte harmonische Punct a gedacht uird, so soll der gemeinsame Ort aller dieser Puncte a bestimmt werden. 
Bemerkung. Ich will hier noch bemerken, dafs ich einige in dieser Abhandlung aufgestellten Sätze nicht genügend bewiesen habe, so dafs dieselben möglicherweise fehlerhaft sein können. Sollte dies der Fall sein, so mag die Neuheit und Schwierigkeit des Gegenstandes, zumal im Vergleich mit der von mir befolgten synthetischen Betrachtungsweise, mich einigermafsen entschuldigen. Namentlich in den drei letzten Paragraphen babe ich

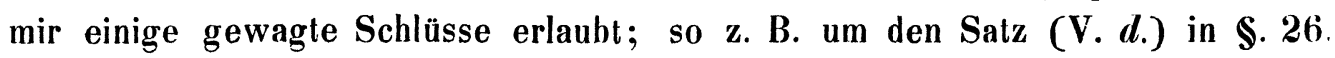
zu erhalten. Ist dieser Satz nicht allgemein wahr, so sind auch mehre ihm vorhergehende Sälze nicht in allen Theilen richtig. 